\title{
Dual Numbers and Invariant Theory of the Euclidean Group \\ with \\ Applications to Robotics \\ Mohammed Daher
}

\section{VICTORIA UNIVERSITY OF WELLINGTON}

Te Whare Wananga o te Upoko o te Ika a Maui

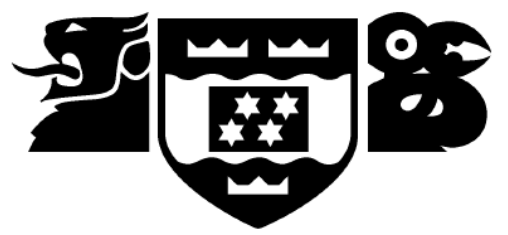

School of Mathematics, Statistics

and Operations Research

Te Kura Mātai Tatauranga, Rangahau Pūnaha

\section{A thesis}

submitted to the Victoria University of Wellington in fulfilment of the requirements for the degree of

Doctor of Philosophy

in Mathematics.

Victoria University of Wellington 



\begin{abstract}
In this thesis we study the special Euclidean group $S E(3)$ from two points of view, algebraic and geometric. From the algebraic point of view we introduce a dualisation procedure for $S O(3, \mathbb{R})$ invariants and obtain vector invariants of the adjoint action of $S E(3)$ acting on multiple screws. In the case of three screws there are 14 basic vector invariants related by two basic syzygies. Moreover, we prove that any invariant of the same group under the same action can be expressed as a rational function evaluated on those 14 vector invariants.

From the geometric point of view, we study the Denavit-Hartenberg parameters used in robotics, and calculate formulae for link lengths and offsets in terms of vector invariants of the adjoint action of $S E(3)$. Moreover, we obtain a geometrical duality between the offsets and the link lengths, where the geometrical dual of an offset is a link length and vice versa.
\end{abstract}





\section{Acknowledgements}

First and foremost, I want to thank my thesis advisor, Dr Peter Donelan, the head of school of mathematics at Victoria University, for a fruitful collaboration during the last three years. He has devoted a lot of his time and patience to our research, while making the process fun and the content accessible.

Thanks are also due to A/Prof Mark McGuiness, my second supervisor, for his help with technical issues.

I am greatly indebted to Dr Petros Hadjicostas for his friendship and many useful discussions and tips regarding my proofs, which at times hinted towards better directions. His brilliant suggestions helped the development of my ideas; many of his technical hints assisted me in mastering the software needed for my research.

I would like to thank Prof Hanspeter Kraft of Basel University, for his guidance in invariant theory.

It was a privilege to study at Victoria University of Wellington, in a suitable place for academic growth. The staff and graduate students provided a friendly and stimulating environment and the administration of SMSOR has ensured the technical requirements for producing my research were met.

I thank Ahmad Abdul-Gaffar, Valentin Bura, Ben Clark, Michael Snook and Michael Welsh for their friendship, encouragement and help with various issues.

On a personal note, I thank my parents and brothers for their inexhaustible financial and emotional support during the last three years. 



\section{Contents}

\begin{tabular}{llr}
\hline & Introduction & 1
\end{tabular}

1.1 The algebraic point of view $\ldots \ldots \ldots$

1.2 The geometric point of view . . . . . . . . . . . . . . 4

1.3 Review of contents $\ldots \ldots \ldots \ldots$

$\begin{array}{lll}2 & \text { Groups and Group Actions } & 13\end{array}$

2.1 The Euclidean group . . . . . . . . . . . . . . . . . . . . 13

2.2 Skew-symmetric matrices . . . . . . . . . . . . . . . . . . . . . 19

2.3 Dual numbers $\ldots \ldots \ldots \ldots$

2.4 Dual matrices . . . . . . . . . . . . . . . . . . . . 22

$3 \quad$ Lie Group and Lie Algebra of $S O(3, \mathbb{R})$ and $S E(3)$

3.1 Preliminaries . . . . . . . . . . . . . . . . . . . . . . . 25

$3.2 \quad$ Lie algebra of $S O(n, \mathbb{R})$ and $S E(n) \ldots \ldots$. . . . . . 27

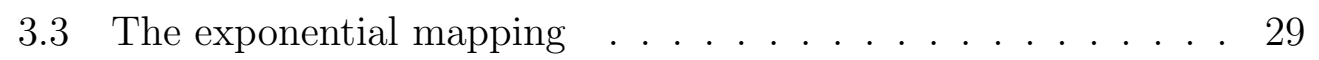

3.4 Adjoint representation $\ldots \ldots \ldots$. . . . . . . . . . . . . 31

3.5 Twists and Plücker coordinates . . . . . . . . . . . . . . . 35

$4 \quad$ Invariant Theory of $S O(3, \mathbb{R})$ and $S E(3)$

4.1 Polynomial rings and rings of invariants. . . . . . . . . . . . . 39

4.2 Vector invariants . . . . . . . . . . . . . . . . . . . 41 
4.3 The first fundamental theorem of invariant theory for $S O(3, \mathbb{R}) 42$

4.4 The second fundamental theorem of invariant theory for $S O(3, \mathbb{R})] \ldots \ldots \ldots \ldots \ldots \ldots$

4.5 The first fundamental theorem of invariant theory for $S E(3)$. 45

$5 \quad$ Algebraic Mappings Among $S O(3, \mathbb{R}), S O(3, \mathbb{D})$ and $S E(3) \quad 47$

5.1 Preliminaries . . . . . . . . . . . . . . . . . . 47

5.2 The algebraic structures on the dual mapping . . . . . . . . 51

5.3 Dual invariants and the special orthogonal group . . . . . . . 53

$6 \quad$ Dualising $S O(3, \mathbb{R})$ Vector Invariants and Syzygies 57

$6.1 \quad$ Dualising the vector invariant of $S O(3, \mathbb{R})$ acting on a single vector . . . . . . . . . . . . . . . . . . 58

6.2 Dualising the vector invariants of $S O(3, \mathbb{R})$ acting on two vectors 58

6.3 Dualising the vector invariants of $S O(3, \mathbb{R})$ acting on three vectors . . . . . . . . . . . . . . . . 61

6.4 Dualising $S O(3, \mathbb{R})$ syzygy $\ldots \ldots \ldots 6$. . . . . . . . . 66

7 Rationally Invariant Functions of the Adjoint Action of $S E(3)$

$\begin{array}{ll}\text { Acting on Triple Screws } & 69\end{array}$

7.1 Introduction . . . . . . . . . . . . . . . . . . . . . . . . . 69

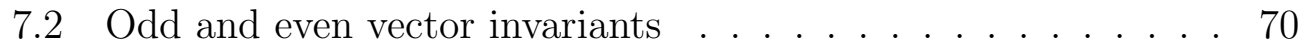

7.3 Choice of a canonical base . . . . . . . . . . . . . . . . . . . 74

$7.4 \quad$ Even invariants of $E(3) \ldots \ldots \ldots . \ldots 76$

7.5 Odd invariants of $E(3)$

$\begin{array}{llr}8 & \text { Application to Robotics } & 85\end{array}$

8.1 Introduction . . . . . . . . . . . . . . . . . . . . . 85

8.2 Denavit-Hartenberg parameters . . . . . . . . . . . 86 
8.3 Link length $\ldots \ldots \ldots \ldots$. . . . . . . . . . . . . . 87

8.4 Offset . . . . . . . . . . . . . . . . . . . 90

9 Geometric Duality for the Set of Three Screws 103

9.1 The geometric interpretation of the Lie bracket of two screws . 103

9.2 Axis of the common perpendicular . . . . . . . . . . . . . . . 104

$9.3 \quad$ Invariants of geometric duals . . . . . . . . . . . . . . 105

9.4 Geometrical duality of offset and link length . . . . . . . . . . 108

9.5 Geometrically dual set of screws . . . . . . . . . . . . . . . 109

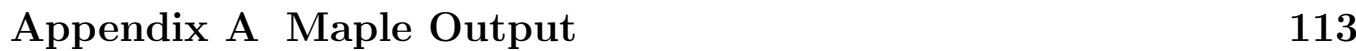

A.1 Rank of algebraically independent vector invariants . . . . . . 113

A.2 Geometric duality between link lengths and offsets . . . . . . . 115

A.3 Even expressions . . . . . . . . . . . . . . . . . . . . . . . . 118

Bibliography 123 


\section{List of Figures}

$3.1 \quad$ A screw line in three dimensional space . . . . . . . . . . . . . 37

8.1 Denavit-Hartenberg frame assignment for serial robot arms . . 86

8.2 The link length between the axis-lines of two screws . . . . . . 88

8.3 Offset between the feet of successive common perpendiculars link lengths. . . . . . . . . . . . . . . . . . . . 91 


\section{Chapter 1}

\section{Introduction}

\subsection{The algebraic point of view}

The aim of this thesis is to completely determine the vector invariant polynomials of the adjoint action of the special Euclidean group $S E(3)$, the isometry group that preserves distance and orientation, by using a technique which depends on dualising (a partial polarisation) vector invariants of the special orthogonal group $S O(3, \mathbb{R})$. There is a Lie algebra isomorphism between $S E(3)$ and the special orthogonal group with dual number entries $S O(3, \mathbb{D})$, and the real and dual parts of all vector invariant polynomials of $S O(3, \mathbb{D})$ will give vector invariant polynomials of $S E(3)$. This approach is guided by the principle of transference which says: any valid proposition about the Lie group $S O(3, \mathbb{R})$ and its Lie algebra becomes on dualisation a valid statement about $S O(3, \mathbb{D})$ and its Lie algebra and hence about $S E(3)$ and its Lie algebra. However, the principle of transference does not give a full guarantee that these vector invariant polynomials, obtained through dualisation, give all vector invariant polynomials of $S E(3)$. Therefore we use the principle of transference as a guidance but should still address a number of questions: 
(a) If we dualise any $S O(3, \mathbb{R})$ vector invariant, do we get an $S O(3, \mathbb{D})$ invariant? Are the real and dual parts $S E(3)$ invariants?

(b) Do the real and dual parts of $S O(3, \mathbb{D})$ vector invariants generate all $S E(3)$ vector invariants in the ring of polynomial invariants, or can we at least express the vector invariants of the adjoint action of $S E(3)$ as rational functions in terms of those vector invariants obtained by dualising $S O(3, \mathbb{R})$ ?

(c) Do the syzygies of vector invariants of $S O(3, \mathbb{R})$ dualise to syzygies of $S O(3, \mathbb{D})$ and hence $S E(3)$ vector invariants?

In this thesis we will address the previous questions and prove some theorems that would help to answer them. However, we need first some basic definitions, theorems, and results found by other mathematicians, so we will state some others' results that might help us in our aim in this research.

In 1900, Hilbert proposed 23 problems that he hoped might be solved during the coming century. Hilbert's fourteenth problem asks whether the algebra of all invariant polynomials under a certain representation is always finitely generated. The ring of invariant polynomials for real reductive groups is now known to be finitely generated [38]. On the other hand, for nonreductive groups we do not have a theorem that the algebra of all invariant polynomials is finitely generated. In fact, we have a counter-example to finite generation. Nagata in 1958 exhibited an example of a non-reductive group for which the ring of invariant polynomials is not finitely generated [36]. More recently, Panyushev [39] gives a general theoretical treatment of Lie algebra semi-direct products from an algebraic-geometric viewpoint. In particular, for semi-direct products $G \ltimes V$ with $G$ reductive group acting on a vector space $V$, the ring of polynomial invariants for the adjoint action is finitely 
generated. In the case $V=\mathfrak{g}$, Panyushev's treatment remains valid up to when syzygies start appearing.

Our key example is the Lie group $S E(3) \cong S O(3, \mathbb{R}) \ltimes \mathbb{R}^{3}$, a semi-direct product of rotations about the origin in $\mathbb{R}^{3}$ with translations, and elements in the Lie algebra $\mathfrak{s e}(3)$ of $S E(3)$ are called screws. The group $S E(3)$ is a non-reductive group, whereas $S O(3, \mathbb{R})$ is a reductive group and Weyl 49 describes the vector invariant theory for the special orthogonal groups in all dimensions. By using Panyushev's treatment mentioned above, the ring of polynomial invariants for the adjoint action of $S E(3)$ acting on single and double screws is finitely generated. Donelan and Gibson [15] prove that every polynomial invariant of the adjoint action of $S E(3)$ belongs to $\mathbb{R}[\boldsymbol{\omega} \cdot \boldsymbol{\omega}, \boldsymbol{\omega} \cdot \boldsymbol{v}]$, see also Donelan and Selig [16]. Crook [12] states the vector invariants of $S E(3)$ acting on double screws and represents them using Plücker coordinates. Selig, in his book [47], Chapter 7 page 161, studies the invariant functions of 3-tensors, or of three screws $S_{u}, S_{v}, S_{w}$ and represents those invariant functions in terms of the invariant $6 \times 6$ symmetric matrices $Q_{\infty}$ and $Q_{0}$. Moreover, the invariants of the adjoint action of $S E(3)$ in Selig's book agree with vector invariants obtained by dualisation in our Theorem 6.3.1. For instance, invariant functions of degree 3 in Selig's book are

$$
S_{u}^{t} Q_{\infty}\left[S_{v}, S_{w}\right], \quad S_{u}^{t} Q_{0}\left[S_{v}, S_{w}\right]
$$

Straightforward calculation can verify that these invariants are equivalent to our $I_{123}$ and $\hat{I}_{123}$ in Theorem 6.3.1. In addition, Study 48, Pages 159 and 202 ] writes down the same invariants and syzygies that we derive using the dualisation procedure in Theorem 6.3.1 and Result 6.4.1. 


\subsection{The geometric point of view}

The Euclidean group $S E(3)$ preserves the distance and orientation in $\mathbb{R}^{3}$, that is, the distance between two points remains invariant and the orientation is preserved, that means, right-handed system of coordinates remains righthanded. As a consequence of the fact that $S E(3)$ satisfy the two previous properties, $S E(3)$ is used for the kinematics of a rigid body. In this thesis we study Denavit-Hartenberg parameters [23] used to describe a serial robot arm. In particular, our interest is in the Denavit-Hartenberg parameters, link length and offset, and we address these questions:

(a) Can we express offset and link length formulae using Plücker coordinates?

(b) Are the offset and the link length parameters invariants of the adjoint action of $S E(3)$ ?

(c) Can we express the offset and the link length formulae in terms of the known vector invariants of the adjoint action of $S E(3)$ ?

In Chapters 8 and 9, we will answer the previous questions and show that there is a new (dual) set of screws whose invariants are related to and expressible in terms of vector invariants of the original set of screws. Now let us review other mathematicians' work in geometry, and in particular, screw theory and its representation.

In a general rigid-body spatial displacement, Chasles [7] described elements of Euclidean group and proved that such a displacement of any element of $S E(3)$ was equivalent to a combination of a rotation about and a translation along some straight line, while Ball [2] was interested in the Lie algebra of the Euclidean group $\mathfrak{s e}(3)$ and had shown that the most general 
velocity of a rigid-body is equivalent to rotation velocity, about a definite axis, combined with a translation velocity along this axis, thus forming a helical motion, which he referred to as a twist velocity about a screw. The screw consists of a screw axis (the same line as the rotation axis) together with a pitch (a linear magnitude) given by the ratio of the magnitude of the translational velocity to the magnitude of the rotational velocity. The twist velocity is hence a screw with an associated magnitude (angular speed).

There is a close connection here with Plücker line coordinates in a 3dimensional projective space, consider a line containing distinct points $\mathbf{x}$ and $\mathbf{y}$ with homogeneous coordinates $\left(x_{0}, x_{1}, x_{2}, x_{3}\right)$ and $\left(y_{0}, y_{1}, y_{2}, y_{3}\right)$, respectively. Let $M$ be the $4 \times 2$ matrix with these coordinates as columns.

$$
M=\left[\begin{array}{ll}
x_{0} & y_{0} \\
x_{1} & y_{1} \\
x_{2} & y_{2} \\
x_{3} & y_{3}
\end{array}\right]
$$

We define Plücker coordinates $p_{i j}$ as the $2 \times 2$ sub-determinant of rows $i$ and $j$ of $M$,

$$
p_{i j}=\left|\begin{array}{ll}
x_{i} & y_{i} \\
x_{j} & y_{j}
\end{array}\right|=x_{i} y_{j}-x_{j} y_{i}
$$

This implies $p_{i i}=0$ and $p_{i j}=-p_{j i}$, reducing the possiblities to only $6=\left(\begin{array}{l}4 \\ 2\end{array}\right)$ distinct quantities $\left(p_{01}, p_{02}, p_{03}, p_{23}, p_{31}, p_{12}\right)$. A line in space can be represented by the six Plücker coordinates which arise as the components of two 3 -vectors $\boldsymbol{\omega}, \boldsymbol{v}[42$ as follows. Assuming the line is not at infinity, so that at least one of $x_{0}, y_{0} \neq 0$, the first vector, $\boldsymbol{\omega}$, with three components, $w_{1}=p_{01}$, 
$w_{2}=p_{02}$, and $w_{3}=p_{03}$, is non-zero and defines the direction of the given line. In this case, the second vector, $\boldsymbol{v}$, with components, $v_{1}=p_{23}, v_{2}=p_{31}$, and $v_{3}=p_{12}$, is the moment of the line about the origin. So, $\boldsymbol{\omega} \times \mathbf{q}=\boldsymbol{v}$, where $\mathbf{q}$ is the position vector of any point on the line. From contemporary standard vector algebra $\boldsymbol{\omega} \cdot \boldsymbol{v}=\boldsymbol{\omega} \cdot(\boldsymbol{\omega} \times \mathbf{q})=0$, and so the two vectors $\boldsymbol{\omega}$ and $\boldsymbol{v}$ are always orthogonal. For finite lines, if we choose $\boldsymbol{\omega}$ to be a unit vector, then six Plücker coordinates satisfy the relations $\boldsymbol{\omega} \cdot \boldsymbol{v}=0$ and $\boldsymbol{\omega} \cdot \boldsymbol{\omega}=1$. There are hence two conditions imposed on the six Plücker coordinates and only four independent coordinates remain. In general, the Plücker coordinates are homogeneous, that is, non-zero multiples of a given set of coordinates represent the same line in space. the equation $\boldsymbol{\omega} \cdot \boldsymbol{v}=0$ defines a fourdimensional quadric hypersurface in projective space $\mathbb{P R}^{5}$, called the Klein quadric. However, points of $\mathbb{P} \mathbb{R}^{5}$ not on the Klein quadric cannot represent lines.

The six Plücker coordinates of a line, define the position and orientation of the line with respect to the origin point. To describe the relative orientation of two skew straight lines in space a unique twist angle, $\alpha$ and a unique common perpendicular distance, $d$, are defined.

The symbol $\epsilon$ in the dual number is originally introduced by Clifford [10]. Study 48 showed how the twist angle, $\alpha$ and common perpendicular distance, $d$, between two skew lines may be combined into a dual number of the form $\alpha+\epsilon d$ (where $\epsilon^{2}=0$ ). In the modern version of Clifford's operator $\epsilon$, lines may be represented using dual vectors. A given line in space, which does not pass through the origin, has three dual angles $\alpha_{x}+\epsilon d_{x}, \alpha_{y}+\epsilon d_{y}$, and $\alpha_{z}+\epsilon d_{z}$, that it makes with the three coordinate axes. These three dual angles may be related to the six Plücker coordinates, Rooney [43] had shown 
that relationships are:

$$
\begin{aligned}
& \cos \left(\alpha_{x}+\epsilon d_{x}\right)=w_{1}+\epsilon v_{1}, \\
& \cos \left(\alpha_{y}+\epsilon d_{y}\right)=w_{2}+\epsilon v_{2}, \\
& \cos \left(\alpha_{z}+\epsilon d_{z}\right)=w_{3}+\epsilon v_{3} .
\end{aligned}
$$

The three dual numbers $w_{1}+\epsilon v_{1}, w_{2}+\epsilon v_{2}$, and $w_{3}+\epsilon v_{3}$ are referred to as the dual direction cosines of the line and they may be considered to be the three components of a unit dual vector. The dual vector describing any line in space is written:

$$
\begin{aligned}
\boldsymbol{\omega}+\epsilon \boldsymbol{v} & =\left(w_{1}, w_{2}, w_{3}\right)+\epsilon\left(v_{1}, v_{2}, v_{3}\right) \\
& =\left(w_{1}+\epsilon v_{1}, w_{2}+\epsilon v_{2}, w_{3}+\epsilon v_{3}\right) .
\end{aligned}
$$

In general, when $\boldsymbol{\omega} \cdot \boldsymbol{v} \neq 0$ the dual vector $\boldsymbol{\omega}+\epsilon \boldsymbol{v}$ represents a screw in space with screw axis $(\boldsymbol{\omega}, \boldsymbol{v}-h \boldsymbol{\omega})$ associated with pitch $h=\frac{\boldsymbol{\omega} \cdot \boldsymbol{v}}{\boldsymbol{\omega} \cdot \boldsymbol{\omega}}$. Dual vectors are another way of representing elements of the Lie algebra $\mathfrak{s e}(3)$ and the screw mentioned in Ball [3].

Clifford [10] extended Hamilton's notion of quaternions and introduced the notion of a biquaternion, which is a combination of two quaternions algebraically combined via a new symbol, $\epsilon$, defined to have the property $\epsilon^{2}=0$. The biquaternion has the form $q+\epsilon r$, where $q$ and $r$ are both quaternions in the usual Hamiltonian form. The dual quaternion is called a motor, which is now known as a screw. Clifford's motivation in creating his biquaternion derives essentially from mechanics and the methods introduced by Clifford are now valuable tools in study of robot manipulators, especially parallel manipulators, see for example Husty 26. 
For sake of completeness we have to mention "the principle of transference", which was originally proven by Kotelnikov but the proof was lost during the Russian revolution [8]. The principle of transference has piqued a lot of mathematicians interest, for instance, Chevallier [8], Rico and Duffy [17, and Study [48]. The principle of transference states that when dual numbers replace real numbers, then all relations of vector algebra for intersecting lines are also valid for skew lines. In practice, this means that all properties of vector algebra for the kinematics of a rigid body with a fixed point (spherical kinematics) also hold for the screw algebra of a free rigid body (spatial kinematics). Consequently, the motion of a general rigid body can be described by only three dual equations rather than six real equations [6].

\subsection{Review of contents}

Now we discuss the structure of this thesis in more detail as follows:

Chapter 2 introduces some background material on the special Euclidean group $S E(3)$ and special orthogonal group $S O(3, \mathbb{R})$, a definition and properties of the dual numbers $\mathbb{D}$, dual matrices, some basic lemmas and theorems that shall help us in proving theorems in the next chapters.

In Chapter 3, we start by defining Lie groups and Lie algebras and describe the algebraic groups $S O(3, \mathbb{R})$ and $S E(3)$ as a Lie groups and their corresponding Lie algebras $\mathfrak{s o}(3, \mathbb{R})$ and $\mathfrak{s e}(3)$ respectively. Since the elements of $\mathfrak{s o}(3, \mathbb{R})$ and $\mathfrak{s e}(3)$ are matrices, the Lie bracket, which is the operation between any two elements in the Lie algebra, is defined by the commutator. We show that the Lie algebra of $\mathfrak{s o}(3, \mathbb{R})$ consists of $3 \times 3$ skew-symmetric matrices. Any element of the Lie algebra of $\mathfrak{s e}(3)$ has been represented using Plücker coordinates $(\boldsymbol{\omega}, \boldsymbol{v})$, where there is an isomorphism between $\mathbb{R}^{3}$ and 
$\mathfrak{s o}(3, \mathbb{R})$ given by the natural representation in Theorem 3.4.1. Moreover, any element of Lie algebra $\mathfrak{s e}(3)$ is called a screw, which is a projective twist. The axis and the pitch of twist are also defined. For the sake of completeness of the introductory material in this chapter, we define the exponential map and how it gives a rise to the adjoint action of the group on its Lie algebra.

Chapter 4 includes the definition of the invariant polynomial that belongs to the ring of polynomials under a certain representation. The adjoint action representation is faithful and hence we can define the vector invariants of the adjoint action of $S E(3)$ acting on screws. Moreover, this chapter includes the first and the second fundamental theorem of invariant theory of $S O(3, \mathbb{R})$ acting on $m$-fold vector and the first fundamental theorem of invariant theory of $S E(3)$ acting on single and double screws.

From now on we start describing the main chapters of this thesis. The remaining five chapters include new material as follows:

In Chapter 5, we prove the isomorphism between $S E(3)$ and $S O(3, \mathbb{D})$ and define the dual map between the ring of real polynomial and the ring of dual polynomial. That enables us to prove that dualising any invariant polynomial of $S O(3, \mathbb{R})$ gives a dual polynomial invariant of $S O(3, \mathbb{D})$. Moreover, we prove that the real and dual part of a dual polynomial, obtained by dualising a polynomial of $S O(3, \mathbb{R})$, are invariant polynomials of $S E(3)$. This result also holds for sets of $k$ vectors.

In Chapter 6, we introduce the process of dualisation. We split the real and the dual part of the dual vector invariants and we obtain the vector invariants of the adjoint action of $S E(3)$ acting on single, double, and triple screws. In the case of three screws, we obtain 14 basic vector invariants. We prove that the first 12 vector invariants are algebraically independent, while the last two, namely $I_{123}$ and $\hat{I}_{123}$, are not algebraically independent with the 
first 12 vector invariants. We find two basic syzygies of $S E(3)$ by dualising $S O(3, \mathbb{R})$ syzygies.

In Chapter 7, we prove every odd and every even vector invariant of the adjoint action of $E(3)$ acting on $k$ screws is a vector invariant of the adjoint action of $S E(3)$ acting on $k$ screws. We show that every even vector invariant of the adjoint action of $E(3)$ acting on triple screws can be expressed rationally in terms of vector invariants $I_{i j}, \hat{I}_{i j}$ and $\hat{I}_{123}^{2}, i=1,2,3$, while we prove that every odd vector invariants of the adjoint action of $E(3)$ acting on triple screws can be expressed by a product $I_{123}$ with even function. Hence the vector invariants of the adjoint action of $S E(3)$ acting on triple screws can be expressed rationally in terms of vector invariants $I_{i j}, \hat{I}_{i j}, I_{123}$ and $\hat{I}_{123}^{2}$, $i=1,2,3$.

In Chapter 8, we introduce Denavit-Hartenberg parameters to define serial robot arms consisting of serial joints. In particular, three serial joints in a general position and ignoring special cases. We focus on two parameters: link length and offset. Obtaining formulae of link lengths and offsets and representing them by using Plücker coordinates is the first step. However, to express the link lengths and the offsets in terms of the vector invariants of the adjoint action of $S E(3)$ is one of the most important results and gives a clearer picture for both the geometers and the algebraists.

Chapter 9 presents the physical interpretation of the Lie bracket of two screws and the consequences of this for sets of three screws. The Lie bracket between two screws gives us a screw whose axis is a common perpendicular to both screws. The set of three screws $S_{i}, i=1,2,3$ has three Lie brackets which means three common perpendicular screws and hence we have a new set of screws $S_{i}^{\prime}, i=1,2,3$. They are related and form a "geometrical duality". We obtain the vector invariants of the adjoint action of 
$S E(3)$ for $S_{i}^{\prime}, i=1,2,3$ and express them in terms of the vector invariants of $S_{i}, i=1,2,3$. Moreover, we discover the relation between $S_{i}$ and $S_{i}^{\prime}$, that is, the link length in $S_{i}$ becomes offset in $S_{i}^{\prime}$ and vice versa. We apply the Lie bracket between any two screws $S_{j}^{\prime}$ and $S_{k}^{\prime}$ and we get the same original screw $S_{i}^{\prime}$ but with different pitch $h_{i}^{\prime \prime}$. 


\section{Chapter 2}

\section{Groups and Group Actions}

\subsection{The Euclidean group}

The set of all $n \times n$ non-singular matrices with elements in a field $\mathbb{k}$ under matrix multiplication forms a group called the general linear group over the field $\mathbb{k}$, denoted by $G L(n, \mathbb{k})$. Unless $n=1$, these groups are not abelian. In this thesis we will only be interested in $\mathbb{k}=\mathbb{R}$.

The special linear group $S L(n, \mathbb{R})$ of order $n$ consists of all those real $n \times n$ matrices $A$ that have inverse and $\operatorname{det} A=1$ and it is a subgroup of $G L(n, \mathbb{R})$. The orthogonal group $O(n, \mathbb{R})$ is also a subgroup of $G L(n, \mathbb{R})$ and is defined as the group of invertible linear transformations that preserve the standard inner product on $\mathbb{R}^{n}$

$$
\langle\mathbf{x}, \mathbf{y}\rangle=\sum_{i=1}^{n} x_{i} y_{i}
$$

That is, for all $\mathbf{x}, \mathbf{y} \in \mathbb{R}^{n}$

$$
\langle A \mathbf{x}, A \mathbf{y}\rangle=\langle\mathbf{x}, \mathbf{y}\rangle .
$$


The orthogonal group has the following well known property

$$
O(n, \mathbb{R})=\left\{A \in G L(n, \mathbb{R}): A^{t} A=I_{n}\right\}
$$

The matrices in the orthogonal group are called orthogonal matrices.

The group $O(n, \mathbb{R})$ consists of two disjoint components-the proper and improper rotations of $\mathbb{R}^{n}$ about the origin. In addition to preserving the inner product (and hence length), proper rotations must also preserve orientation. A non-singular matrix will preserve or reverse orientation according to whether the determinant of the matrix is positive or negative. Explicitly, for an orthogonal matrix $A$, note that $\operatorname{det} A^{t}=\operatorname{det} A \operatorname{implies}(\operatorname{det} A)^{2}=1$ so that $\operatorname{det} A= \pm 1$. The subgroup of orthogonal matrices with determinant +1 that preserve orientation is called the special orthogonal group and is denoted by $S O(n, \mathbb{R})$. That is,

$$
S O(n, \mathbb{R})=\left\{A \in G L(n, \mathbb{R}): A^{t} A=I, \operatorname{det} A=1\right\}
$$

Applications in physics and engineering frequently concern rigid bodies in 3-dimensions and so $S O(3, \mathbb{R})$ is of particular importance.

Definition 2.1.1. A group $G$ with identity $e$ acts on a set $X$ if there is a function $\rho: G \times X \longrightarrow X$ (called an action of $G$ ) such that:

(a) $\rho(e, x)=x$ for all $x \in X$;

(b) $\rho(g h, x)=\rho(g, \rho(h, x))$ for all $g, h \in G$ and $x \in X$.

By saying that a group $G$ of matrices is a collection of symmetries of a set $X \subseteq \mathbb{R}^{n}$ we mean that there is an action $\rho$ of $G$ on $X$ defined via matrixvector multiplication. For instance, given $A \in S O(n, \mathbb{R})$ and $\mathbf{x} \in \mathbb{R}^{n}$, this may define $\rho(A, \mathbf{x})=A \mathbf{x}$. 
The usual Euclidean metric for any $\mathbf{x}, \mathbf{y} \in \mathbb{R}^{n}$ is defined by

$$
d(\mathbf{x}, \mathbf{y})=\|\mathbf{x}, \mathbf{y}\|=\sqrt{\langle\mathbf{x}-\mathbf{y}, \mathbf{x}-\mathbf{y}\rangle} .
$$

The Euclidean group (of order $n$ ) $E(n)$ is the symmetry group of the $n$ dimensional Euclidean space, that is, the set of isometries $\mu$ of $\mathbb{R}^{n}$ with the Euclidean metric

$$
\begin{aligned}
& \mu: \mathbb{R}^{n} \longrightarrow \mathbb{R}^{n} \\
& d(\mu(\mathbf{x}), \mu(\mathbf{y}))=d(\mathbf{x}, \mathbf{y}) .
\end{aligned}
$$

It is clear that any isometry is a bijection and that its inverse is also an isometry. Moreover $E(n)$ is closed under composition and so certainly is a group. Such isometries are called Euclidean motions.

Theorem 2.1.2. Every isometry $\mu$ of $\mathbb{R}^{n}$ can be achieved by an orthogonal transformation $A \in O(n, \mathbb{R})$ about the origin followed by a translation $\mathbf{a} \in$ $\mathbb{R}^{n}$

Proof. See [7].

Hence, as a set, $E(n)$ is in bijective correspondence with the Cartesian product

$$
O(n, \mathbb{R}) \times \mathbb{R}^{n}
$$

However, as a group, the isomorphism is with the semi-direct product. The composition of two isometries $\mu_{i}$ represented by $\left(A_{i}, \mathbf{a}_{i}\right) \in O(n, \mathbb{R}) \times \mathbb{R}^{n}$, 
where $i=1,2$ is:

$$
\begin{aligned}
\mu_{2} \circ \mu_{1}(\mathbf{x}) & =A_{2}\left(A_{1} \mathbf{x}+\mathbf{a}_{1}\right)+\mathbf{a}_{2} \\
& =A_{2} A_{1} \mathbf{x}+A_{2} \mathbf{a}_{1}+\mathbf{a}_{2} .
\end{aligned}
$$

Hence composition in $O(n, \mathbb{R}) \times \mathbb{R}^{n}$ is

$$
\left(A_{2}, \mathbf{a}_{2}\right)\left(A_{1}, \mathbf{a}_{1}\right)=\left(A_{2} A_{1}, A_{2} \mathbf{a}_{1}+\mathbf{a}_{2}\right) .
$$

The composition of any two elements in $E(n)$ given in 2.10 tells us the structure of this group, since the first component is a direct product but the second not. This means the structure of $E(n)$ is a semidirect or twisted product of rotation group $O(n, \mathbb{R})$ with the translation group $\mathbb{R}^{n}$ :

$$
E(n) \cong O(n, \mathbb{R}) \ltimes \mathbb{R}^{n}
$$

Such structures arise when there is an action of a group $G$ on a vector space $V$ and we obtain $G \ltimes V$ as a group.

There is a subgroup $S E(n)$ of the Euclidean group, special Euclidean group of order $n$, consisting of the direct isometries, that is, isometries preserving orientation, the orthogonal part has determinant equal one. By Theorem 2.1.2 that subgroup consists of rotation about the origin and translation, that is,

$$
S E(n) \cong S O(n, \mathbb{R}) \ltimes \mathbb{R}^{n}
$$

A representation is a homomorphism from the group to general linear group. Note that $S E(n)$ is not defined as a matrix group. However, there 
is a $(n+1)$-dimensional representation of $S E(n)$, that is, an injective homomorphism $S E(n) \longrightarrow G L(n+1)$ given by

$$
(A, \mathbf{a}) \mapsto\left(\begin{array}{cc}
A & \mathbf{a} \\
\mathbf{0}^{t} & 1
\end{array}\right)
$$

We have used a partitioned form for the matrix on the right and we will use $\mathbf{0}$ to mean $\mathbf{0}^{t}$ to make the notation simpler. This representation is a homomorphism, since multiplying these matrices exactly replicates the product of the pairs in 2.10

$$
\left(\begin{array}{cc}
A_{2} & \mathbf{a}_{2} \\
\mathbf{0} & 1
\end{array}\right)\left(\begin{array}{cc}
A_{1} & \mathbf{a}_{1} \\
\mathbf{0} & 1
\end{array}\right)=\left(\begin{array}{cc}
A_{2} A_{1} & A_{2} \mathbf{a}_{1}+\mathbf{a}_{2} \\
\mathbf{0} & 1
\end{array}\right)
$$

The inverse of such a matrix is conveniently given by

$$
\left(\begin{array}{ll}
A & \mathbf{a} \\
\mathbf{0} & 1
\end{array}\right)^{-1}=\left(\begin{array}{cc}
A^{t} & -A^{t} \mathbf{a} \\
\mathbf{0} & 1
\end{array}\right)
$$

Of interest in the special Euclidean group is when $n=3$, that is $S E(3)$. This group has applications in physics and engineering. In this thesis we shall study some applications of this group to robotics. For example, suppose we want to study a moving rigid body, such as the end-effector of a robot arm. We can specify two different coordinate frames: a home frame of reference that is fixed in the ambient apace around the rigid body, and a coordinate system embedded in the body itself. If a point has coordinates $\mathbf{p}=(x, y, z)$ in the reference frame, and coordinates $\mathbf{p}^{\prime}=\left(x^{\prime}, y^{\prime}, z^{\prime}\right)$ in the body frame, 
then these are linked by

$$
\left(\begin{array}{l}
\mathbf{p}^{\prime} \\
1
\end{array}\right)=\left(\begin{array}{ll}
A & \mathbf{a} \\
\mathbf{0} & 1
\end{array}\right)\left(\begin{array}{l}
\mathbf{p} \\
1
\end{array}\right),
$$

where $(A, \mathbf{a})$ is the Euclidean motion with respect to choice of coordinates. At the same time, assuming we want the coordinate system to coincide with the home position of the end-effector, the end-effector coordinates are transformed in exactly the same way. Now suppose, we choose different home coordinates. they are related to the previous ones by

$$
\left(\begin{array}{l}
\mathbf{q} \\
1
\end{array}\right)=\left(\begin{array}{ll}
B & \mathbf{b} \\
\mathbf{0} & 1
\end{array}\right)\left(\begin{array}{l}
\mathbf{p} \\
1
\end{array}\right)
$$

Then by using (2.16) and multiplying by the inverse of both sides in (2.17), the motion in new coordinates is

$$
\begin{aligned}
\left(\begin{array}{l}
\mathbf{q}^{\prime} \\
1
\end{array}\right) & =\left(\begin{array}{ll}
B & \mathbf{b} \\
\mathbf{0} & 1
\end{array}\right)\left(\begin{array}{l}
\mathbf{p}^{\prime} \\
1
\end{array}\right) \\
& =\left(\begin{array}{ll}
B & \mathbf{b} \\
\mathbf{0} & 1
\end{array}\right)\left(\begin{array}{ll}
A & \mathbf{a} \\
\mathbf{0} & 1
\end{array}\right)\left(\begin{array}{ll}
B & \mathbf{b} \\
\mathbf{0} & 1
\end{array}\right)^{-1}\left(\begin{array}{l}
\mathbf{q} \\
1
\end{array}\right) .
\end{aligned}
$$

So the transformation in the new coordinates is given by the conjugate of the original transformation. In fact, in any group $G$ conjugation can be thought of as an action $\rho$ of $G$ on itself

$$
\rho(h, g)=h g h^{-1}
$$




\subsection{Skew-symmetric matrices}

A skew-symmetric (or antisymmetric) matrix is a square matrix $T$ that satisfies $T=-T^{t}$. If the entry in the $i^{t h}$ row and $j^{t h}$ column is $a_{i j}$, then $a_{i j}=-a_{j i}$. In particular all the main diagonal entries of skew-symmetric matrix must be zero, so the trace is zero. Sums and scalar multiples of skew-symmetric matrices are again skew-symmetric. Hence, the $n \times n$ skewsymmetric matrices form a vector space, whose dimension is $n(n-1) / 2$.

Lemma 2.2.1. If $T$ is an $n \times n$ skew-symmetric matrix, then for any $\mathbf{x} \in \mathbb{R}^{n}$, $\mathbf{x}^{t} T \mathbf{x}=0$.

Proof. Any an $n \times n$ skew-symmetric matrix $T$ satisfies $T=-T^{t}$, that implies to $T+T^{t}=0$, therefore $\mathbf{x}^{t}\left(T+T^{t}\right) \mathbf{x}=0$ and hence $\mathbf{x}^{t} T \mathbf{x}=0$.

\subsection{Dual numbers}

Dual numbers, $\mathbb{D}$, are a 2-dimensional commutative algebra over reals $a$, and $b$ and have the form $\hat{d}=a+\epsilon b$, where $\epsilon$ is called the $d u a l$ unit and has properties

$$
\epsilon \neq 0, \quad 0 \epsilon=\epsilon 0=0, \quad 1 \epsilon=\epsilon 1=\epsilon, \quad \epsilon^{2}=0 .
$$

Dual numbers were first proposed by Clifford $[9]$ and developed by the German geometer E. Study (1862-1930) [48]. Study used dual numbers to represent the relative position of two skew lines in space. This is a dual angle which is defined as:

$$
\hat{\alpha}=\alpha+\epsilon d
$$

where $d$ is the length of the common perpendicular to the two lines in three 
dimensional space and $\alpha$ is the projective angle between the two lines.

The dual numbers form a commutative ring isomorphic to the quotient of the polynomial ring $\mathbb{R}[x]$ by the ideal generated by the polynomial $x^{2}$

$$
\mathbb{R}[x] /\left\langle x^{2}\right\rangle .
$$

The image of $x$ in the quotient corresponds to the dual unit $\epsilon$, the dual numbers form a commutative ring with characteristic 0 . While the inherited multiplication gives the dual numbers the structure of a commutative and associative algebra of dimension two over the reals, they do not form a field since there is no multiplicative inverse.

The operations of addition and multiplication are defined for any two dual numbers $\hat{d}_{1}=a_{1}+\epsilon b_{1}$ and $\hat{d}_{2}=a_{2}+\epsilon b_{2}$ :

$$
\left(a_{1}+\epsilon b_{1}\right)+\left(a_{2}+\epsilon b_{2}\right)=\left(a_{1}+a_{2}\right)+\epsilon\left(b_{1}+b_{2}\right)
$$

$\mathbb{D}$ becomes an associative algebra with multiplication defined by setting $\epsilon^{2}=$ 0 , thus

$$
\left(a_{1}+\epsilon b_{1}\right)\left(a_{2}+\epsilon b_{2}\right)=a_{1} a_{2}+\epsilon\left(a_{1} b_{2}+a_{2} b_{1}\right)
$$

provided that $a_{2} \neq 0$, the quotient $\frac{\hat{d}_{1}}{\hat{d}_{2}}$ is also defined for all $\hat{d}_{1}$ and $\hat{d}_{2}$ as follows:

$$
\frac{\hat{d}_{1}}{\hat{d}_{2}}=\frac{a_{1}+\epsilon b_{1}}{a_{2}+\epsilon b_{2}}=\frac{a_{1}}{a_{2}}+\epsilon\left(\frac{b_{1}}{a_{2}}-\frac{a_{1} b_{2}}{a_{2}^{2}}\right) .
$$


The conjugate of a dual number $\hat{d}$ is defined by:

$$
\overline{\hat{d}}=a-\epsilon b .
$$

The product of a dual number $\hat{d}$ and its conjugate $\overline{\hat{d}}$ satisfies:

$$
\hat{d} \overline{\hat{d}}=a^{2}
$$

The modulus of a dual number is simply the real part

$$
|\hat{d}|=a
$$

The modulus can be negative since $a$ is real number.

If $f: \mathbb{R} \mapsto \mathbb{R}$ is a polynomial function or given by a power series, then we can define $\hat{f}: \mathbb{D} \mapsto \mathbb{D}$ by replacing the real variable by a dual quantity $\hat{d}=a+\epsilon b$, that is,

$$
\hat{f}(\hat{d})=f(a+\epsilon b) .
$$

Let $f(x)=x^{3}$ so

$$
\hat{f}(a+\epsilon b)=(a+\epsilon b)^{3}=a^{3}+3 \epsilon a^{2} b .
$$

Now consider $f$ as a power series in $\epsilon$ so

$$
\hat{f}(\hat{d})=f(a)+\epsilon b f^{\prime}(a), \quad \text { since } \epsilon^{2}=0 .
$$

This property is useful in the expansion of polynomials and power series. For 
example,

$$
\begin{aligned}
& \sin \hat{\alpha}=\sin (\alpha+\epsilon d)=\sin \alpha+\epsilon d \cos \alpha, \\
& \cos \hat{\alpha}=\cos (\alpha+\epsilon d)=\cos \alpha-\epsilon d \sin \alpha, \\
& e^{a+\epsilon b}=e^{a}(1+\epsilon b) .
\end{aligned}
$$

\subsection{Dual matrices}

Any $(n \times m)$ matrix, whose entries belong to the reals, can be written as $A=\left(a_{i j}\right)$ where $a_{i j} \in \mathbb{R}$ but if we replace the entries by dual numbers $\left(\hat{a}_{i j}\right) \in \mathbb{D}$ then the last is called a dual matrix.

Lemma 2.4.1. If $\hat{A}$ is a $(n \times m)$ matrix with dual number entries, then we can write $\hat{A}$ as $\hat{A}=A_{0}+\epsilon A_{1}$ where $A_{0}, A_{1}$ are $(n \times m)$ matrices belonging to $M_{\mathbb{R}}(m, n)$.

Proof. Assume $\hat{A}$ is a $(n \times m)$ matrix with dual numbers entries $\left(\hat{a}_{i j}\right) \in \mathbb{D}$, hence $\hat{a}_{i j}=a_{i j}+\epsilon b_{i j}$ where $\left(a_{i j}\right),\left(b_{i j}\right) \in \mathbb{R}$

$$
\begin{aligned}
\hat{A} & =\left[\begin{array}{cccc}
\hat{a}_{11} & \hat{a}_{12} & \cdots & \hat{a}_{1 n} \\
\hat{a}_{21} & \hat{a}_{22} & \cdots & \hat{a}_{2 n} \\
\vdots & \vdots & \ddots & \vdots \\
\hat{a}_{m 1} & \hat{a}_{m 2} & \cdots & \hat{a}_{m n}
\end{array}\right] \\
= & {\left[\begin{array}{cccc}
a_{11}+\epsilon b_{11} & a_{12}+\epsilon b_{12} & \cdots & a_{1 n}+\epsilon b_{1 n} \\
a_{21}+\epsilon b_{21} & a_{22}+\epsilon b_{22} & \cdots & a_{2 n}+\epsilon b_{2 n} \\
\vdots & \vdots & \ddots & \vdots \\
a_{m 1}+\epsilon b_{m 1} & a_{m 2}+\epsilon b_{m 2} & \cdots & a_{m n}+\epsilon b_{m n}
\end{array}\right] }
\end{aligned}
$$




$$
\begin{aligned}
& =\left[\begin{array}{cccc}
a_{11} & a_{12} & \cdots & a_{1 n} \\
a_{21} & a_{22} & \cdots & a_{2 n} \\
\vdots & \vdots & \ddots & \vdots \\
a_{m 1} & a_{m 2} & \cdots & a_{m n}
\end{array}\right]+\epsilon\left[\begin{array}{cccc}
b_{11} & b_{12} & \cdots & b_{1 n} \\
b_{21} & b_{22} & \cdots & b_{2 n} \\
\vdots & \vdots & \ddots & \vdots \\
b_{m 1} & b_{m 2} & \cdots & b_{m n}
\end{array}\right] \\
= & A_{0}+\epsilon A_{1} .
\end{aligned}
$$

Thus, any dual matrix can be written using this form $\hat{A}=A_{0}+\epsilon A_{1}$.

Lemma 2.4.2. If $\hat{A}$ is a $(m \times n)$ dual matrix, then the transpose of $\hat{A}$ is the $(n \times m)$ dual matrix, and can be written as $\hat{A}^{t}=\left(A_{0}+\epsilon A_{1}\right)^{t}=A_{0}^{t}+\epsilon A_{1}^{t}$.

Proof. From linear algebra, the transpose of summation of any two matrices is the sum of the transpose of each one, that is $(A+B)^{t}=A^{t}+B^{t}$, and the transpose of scalar multiple matrix is the scalar times matrix transpose, that is $(\lambda B)^{t}=\lambda B^{t}$, when the two previous properties on the transpose of dual matrix are applied the result is $\hat{A}^{t}=\left(A_{0}+\epsilon A_{1}\right)^{t}=A_{0}^{t}+\epsilon A_{1}^{t}$.

Definition 2.4.3. (McCarthy 34 ). If $\hat{A}$ is a $(n \times n)$ matrix with dual number entries, $\hat{A}=A_{0}+\epsilon A_{1}$, where $A_{0}, A_{1} \in M(n, \mathbb{R})$ then the dual orthogonal group is

$$
S O(n, \mathbb{D})=\left\{\hat{A} \in M(n, \mathbb{D}) \quad \mid \quad \hat{A}^{t} \hat{A}=I_{n}, \operatorname{det} \hat{A}=1\right\}
$$

Theorem 2.4.4. Any element belonging to $S O(n, \mathbb{D})$ can be written as $\hat{A}=$ $A_{0}+\epsilon A_{1}$ if and only if $A_{0} \in S O(n, \mathbb{R})$ and $\left(A_{0}^{t} A_{1}\right)$ is skew-symmetric matrix.

Proof. Let $\hat{A}$ be a matrix belonging to $S O(n, \mathbb{D})$, which must satisfy $S O(n, \mathbb{D})$ properties in Definition 2.4.3. $\hat{A}$ can be written as $\hat{A}=A_{0}+\epsilon A_{1}$, then

$$
\hat{A}^{t} \hat{A}=\left(A_{0}^{t}+\epsilon A_{1}^{t}\right)\left(A_{0}+\epsilon A_{1}\right)=A_{0}^{t} A_{0}+\epsilon\left(A_{0}^{t} A_{1}+A_{1}^{t} A_{0}\right)=I .
$$


This implies $A_{0}^{t} A_{0}=I$, which means $A_{0}$ is an orthogonal matrix. Moreover,

$$
A_{0}^{t} A_{1}+A_{1}^{t} A_{0}=0_{n \times n} \Longrightarrow A_{0}^{t} A_{1}=-A_{1}^{t} A_{0} \Longrightarrow A_{0}^{t} A_{1}=-\left(A_{0}^{t} A_{1}\right)^{t}
$$

which means $A_{0}^{t} A_{1}$ is skew-symmetric matrix. We have

$$
\begin{aligned}
\operatorname{det}\left(\hat{A}^{t} \hat{A}\right) & =\operatorname{det}(A_{0}^{t} A_{0}+\epsilon \underbrace{\left(A_{0}^{t} A_{1}+A_{1}^{t} A_{0}\right)}_{0_{n \times n}}) \\
& =\operatorname{det}\left(A_{0}^{t} A_{0}\right)=\operatorname{det}\left(A_{0}\right)^{2}=\left(\operatorname{det} A_{0}\right)^{2}=1,
\end{aligned}
$$

The fact that $\operatorname{det}(\hat{A})=1$ implies to $\operatorname{det}\left(A_{0}\right)=1$, and hence $A_{0} \in S O(n, \mathbb{R})$. The reverse follows by reversing this argument. 


\section{Chapter 3}

\section{Lie Group and Lie Algebra of $S O(3, \mathbb{R})$ and $S E(3)$}

\subsection{Preliminaries}

A real Lie group is a group that is also a smooth real finite-dimensional manifold, so that the multiplication function $G \times G \longrightarrow G:(a, b) \mapsto a b$ and the inverse function $G \longrightarrow G: a \mapsto a^{-1}$ are smooth maps. $G L(n, \mathbb{R})$, $S O(n, \mathbb{R})$, and $S E(n)$ are examples of real Lie groups.

To identify an element of the tangent space of a Lie group, consider a smooth path through the identity in a group $G$, that is $p: \mathbb{R} \longrightarrow G$ such that $p(0)=I$, then $\frac{d}{d t}[p(t)]_{t=0} \in T_{I} G$. If the first derivative of paths at 0 are the same, then those paths are considered equivalent. A tangent vector is an equivalence class for this relation. The space of equivalence classes can be shown to be a vector space.

The product between two paths in the group is defined as $p(t) q(s) \in G$. However, to understand the influence of the product on the group in the tangent space of the same group, we should consider these two paths $p, q$ : 
$\mathbb{R} \longrightarrow G$ and find the first derivative and assume that:

$$
\frac{d}{d t}[p(t)]_{t=0}=Y, \quad \frac{d}{d s}[q(s)]_{s=0}=X
$$

We should differentiate the product with respect to time $t$, and then with respect to time $s$ in order to obtain a tangent vector at the identity $I$ we have to apply the conjugation trick, that is, $q(s) p(t) q(s)^{-1}$, and remember that $q(0)=p(0)=I$, then

$$
\begin{aligned}
\frac{d}{d s}\left[\frac{d}{d t}\left[q(s) p(t) q(s)^{-1}\right]_{t=0}\right]_{s=0} & =\frac{d}{d s}\left[q(s) Y q(s)^{-1}\right]_{s=0} \\
& =\frac{d}{d s}[q(s)]_{s=0} Y q(0)^{-1}+q(0) Y \frac{d}{d s}\left[q(s)^{-1}\right]_{s=0}
\end{aligned}
$$

If we differentiate $q(s) q(s)^{-1}=I$ with respect to time $s$ and evaluate at $s=0$, then we get $\frac{d}{d s}\left[q(s)^{-1}\right]_{s=0}=-\frac{d}{d s}[q(s)]_{s=0}=-X$, and hence

$$
=X Y-Y X
$$

This is the commutator of $X$ and $Y$ for the tangent space of a Lie Group is called the Lie bracket, where $X Y$ denotes the standard matrix product. It is a binary operator $[\cdot, \cdot]$ assigning an element of the tangent space $T_{I} G$ to any pair of elements. The Lie bracket satisfies the following properties:

(a) bilinear: $\left[\lambda X_{1}+\beta X_{2}, Y\right]=\lambda\left[X_{1}, Y\right]+\beta\left[X_{2}, Y\right]$ and similarly for the second argument.

(b) anti-symmetric: for all $X, Y: \quad[X, Y]=-[Y, X]$

(c) Jacobi identity: for all $X, Y, Z$

$$
[X,[Y, Z]]+[Y,[Z, X]]+[Z,[X, Y]]=0
$$


A Lie algebra is a vector space $\mathfrak{g}$ over a field $\mathbb{k}$ equipped with a skewsymmetric $\mathbb{k}$-bilinear form $[\cdot, \cdot]: \mathfrak{g} \times \mathfrak{g} \longrightarrow \mathfrak{g}$, which satisfies the Jacobi identity.

\subsection{Lie algebra of $S O(n, \mathbb{R})$ and $S E(n)$}

To find Lie algebra for orthogonal groups, consider the tangent space to the identity in $O(n, \mathbb{R})$ and $S O(n, \mathbb{R})$. The orthogonal group $O(n, \mathbb{R})$ consists of two disconnected components, the one that contains the identity, is just $S O(n, \mathbb{R})$. So $O(n, \mathbb{R})$ and $S O(n, \mathbb{R})$ have the same tangent space at the identity and hence the same Lie algebra. In a matrix representation of $S O(n, \mathbb{R})$ a path through the identity is given by a matrix-valued function, $\gamma(t)=M(t)$, where $M(0)=I_{n}$ and by 2.3

$$
M(t)^{t} M(t)=I_{n}
$$

Differentiating the last relation with respect to $t$, gives

$$
\left(\frac{d}{d t} M(t)^{t}\right) M(t)+M(t)^{t}\left(\frac{d}{d t} M(t)\right)=0
$$

Putting $t=0$ we get

$$
\dot{M}(0)^{t}+\dot{M}(0)=0
$$

The last equation tells us that $X=\dot{M}(0)$ is a skew-symmetric matrix (i.e. $\left.X^{t}=-X\right)$. Hence, the tangent space to the identity $I_{n} \in S O(n, \mathbb{R})$ consists 
of skew-symmetric matrices. Thus

$$
\mathfrak{s o}(n, \mathbb{R})=\left\{X \quad \mid \quad X+X^{t}=0_{n \times n}\right\} .
$$

As a special case, when $n=3$, the Lie algebra of the special orthogonal group denoted by $\mathfrak{s o}(3, \mathbb{R})$ and consists of $3 \times 3$ skew-symmetric matrices as follows:

$$
=\left\{\left(\begin{array}{ccc}
0 & -t_{3} & t_{2} \\
t_{3} & 0 & -t_{1} \\
-t_{2} & t_{1} & 0
\end{array}\right) \quad \mid \quad t_{1}, t_{2}, t_{3} \in \mathbb{R}\right\} .
$$

To find the tangent space to the identity in $S E(n)$, consider this path

$$
\gamma: t \longmapsto\left(\begin{array}{cc}
A(t) & a(t) \\
\mathbf{0} & 1
\end{array}\right)
$$

This is a curve in the group $S E(n)$, parametrised by time $t$. The derivative at the identity element will thus be of the form

$$
\left(\begin{array}{ll}
B & \mathbf{b} \\
\mathbf{0} & 0
\end{array}\right)
$$

where $B$ is a $n \times n$ skew-symmetric matrix and $\mathbf{b}$ a $n$-component vector. Hence

$$
\mathfrak{s e}(n, \mathbb{R}) \cong\left\{(B, \mathbf{b}) \quad \mid \quad B \in \mathfrak{s o}(n, \mathbb{R}), \mathbf{b} \in \mathbb{R}^{n}\right\}
$$

Tangent spaces give us a simple way to find the dimension of the group, since the dimension of the manifold is the same as the dimension of its tangent space. The dimension of the groups $O(n, \mathbb{R})$ and $S O(n, \mathbb{R})$ is thus the 
dimension of the vector space of $n \times n$ skew-symmetric matrices, which is $\frac{1}{2} n(n-1)$. However, the dimension of $S E(n)$ is $n(n+1) / 2$, where $n$ can be attributed to the dimension of $\mathbb{R}^{n}$, and the remaining $n(n-1) / 2$ is the dimension of $S O(n, \mathbb{R})$.

\subsection{The exponential mapping}

Another way of looking at Lie algebra elements is as left-invariant vector fields on the group. Given a tangent vector at the identity, we can produce a left-invariant vector field. All we do is to left translate the original vector to every point on the group. If $X$ is a matrix representing a tangent vector at the identity, then the tangent vector at the point $g$ of the group will be given by $g X$. Hence, there is a one-to-one correspondence between tangent vectors at the identity and left-invariant vector fields. Integral curves of a vector field are smooth curves on manifold whose tangent vector at each point is in a vector field at each of its points, such a curve would satisfy the differential equation

$$
\frac{d \gamma}{d t}=\gamma X
$$

This equation has an analytic solution that passes through the identity

$$
\gamma(t)=e^{t X}
$$

In the case that $G$ is a matrix group, the exponential of matrix $X$ can be expanded into a power series:

$$
e^{X}=1+X+\frac{X^{2}}{2 !}+\cdots+\frac{X^{n}}{n !}+\cdots
$$


The exponential function gives a mapping from the Lie algebra to the group. In general this mapping is neither one-to-one nor onto. However, the following theorem gives locally one-to one and onto. Moreover, there is a neighbourhood of 0 in Lie algebra that maps homeomorphically to the neighbourhood of the identity in the group.

Theorem 3.3.1. (Hall [22]). Let $G$ be a matrix Lie group with Lie algebra $\mathfrak{g}$. Then there exists a neighbourhood $U$ of zero in $\mathfrak{g}$ and a neighbourhood $U^{\prime}$ of the identity in $G$ such that the exponential mapping takes $U$ homeomorphically onto $U^{\prime}$.

Proof. See chapter three in [22].

As a special case the Lie algebra $\mathfrak{s o}(3, \mathbb{R})$ has three elements that are defined as

$$
J_{x}=\left[\begin{array}{ccc}
0 & 0 & 0 \\
0 & 0 & -1 \\
0 & 1 & 0
\end{array}\right], \quad J_{y}=\left[\begin{array}{ccc}
0 & 0 & 1 \\
0 & 0 & 0 \\
-1 & 0 & 0
\end{array}\right], \quad J_{z}=\left[\begin{array}{ccc}
0 & -1 & 0 \\
1 & 0 & 0 \\
0 & 0 & 0
\end{array}\right]
$$

In fact the Lie bracket cyclically permutes the basis elements

$$
\left[J_{x}, J_{y}\right]=J_{z}, \quad\left[J_{y}, J_{z}\right]=J_{x}, \quad\left[J_{z}, J_{x}\right]=J_{y} .
$$

We can also explicitly describe the corresponding subgroups in $S O(3, \mathbb{R})$ as

$$
\exp \left(t J_{x}\right)=\left[\begin{array}{ccc}
1 & 0 & 0 \\
0 & \cos t & -\sin t \\
0 & \sin t & \cos t
\end{array}\right], \exp \left(t J_{y}\right)=\left[\begin{array}{ccc}
\cos t & 0 & \sin t \\
0 & 1 & 0 \\
-\sin t & 0 & \cos t
\end{array}\right], \exp \left(t J_{z}\right)=\left[\begin{array}{ccc}
\cos t & -\sin t & 0 \\
\sin t & \cos t & 0 \\
0 & 0 & 1
\end{array}\right]
$$

The matrix $\exp \left(t J_{x}\right)$ is a rotation around the $x$-axis by angle $t$. Similarly $J_{y}$ and $J_{z}$ generate rotations around the $y$ and $z$ axes respectively. In fact 
since $S O(3, \mathbb{R})$ is compact the three elements generate a neighbourhood of identity in $S O(3, \mathbb{R})$, and hence they generate the whole group $S O(3, \mathbb{R})$.

\subsection{Adjoint representation}

There is a natural representation of the group on its Lie algebra called the adjoint representation of the group. Consider a Lie group $G$ and the conjugation by an element $g \in G$. This gives a smooth mapping from the manifold of $G$ back to itself. A simple path in the group is given in a matrix representation by

$$
\gamma: t \mapsto I+t X+t^{2} Q(t)
$$

where $X$ is the Lie algebra element and $Q(t)$ the remainder that ensures that the image of the path stays in the group. If we conjugate by $g$, then differentiate and set $t=0$, we get $g X g^{-1}$. So the action for all $g \in G$ is given by

$$
A d(g) X=g X g^{-1}
$$

This action is called the adjoint action and it is linear since for any two scalars $\alpha$ and $\beta$, we have

$$
\begin{aligned}
A d(g)\left(\alpha X_{1}+\beta X_{2}\right)=g\left(\alpha X_{1}+\beta X_{2}\right) g^{-1} & =\alpha g X_{1} g^{-1}+\beta g X_{2} g^{-1} \\
& =\alpha A d(g) X_{1}+\beta A d(g) X_{2}
\end{aligned}
$$


To find the adjoint action of $\mathfrak{s o}(3)$, we must calculate the product

$$
\Omega^{\prime}=R \Omega R^{t}
$$

where $R \in S O(3, \mathbb{R})$ and $\Omega$ is $3 \times 3$ skew-symmetric matrix. To facilitate the computation, we will write the rotation matrix as partitioned into three vectors:

$$
R=\left(\begin{array}{c}
\mathbf{r}_{1}^{t} \\
\mathbf{r}_{2}^{t} \\
\mathbf{r}_{3}^{t}
\end{array}\right)
$$

Because of $R R^{t}=I_{3}$, the vectors must be mutually orthogonal unit vectors $\mathbf{r}_{i} \mathbf{r}_{j}=1$ if $i=j$, and $\mathbf{r}_{i} \mathbf{r}_{j}=0$ if $i \neq j$. Moreover, $\operatorname{det}(R)=1$ means that the triple product

$$
\mathbf{r}_{1} \cdot\left(\mathbf{r}_{2} \times \mathbf{r}_{3}\right)=1
$$

If we multiply both sides of Equation 3.19 by $\mathbf{r}_{1}$, and permute 3.19 cyclically to have $\mathbf{r}_{2} \cdot\left(\mathbf{r}_{3} \times \mathbf{r}_{1}\right)=1$ and multiply both sides by $\mathbf{r}_{2}$, and similarly permute again the same equation to get $\mathbf{r}_{3} \cdot\left(\mathbf{r}_{1} \times \mathbf{r}_{2}\right)=1$ and multiply again by $\mathbf{r}_{3}$, then we get these three equations

$$
\mathbf{r}_{2} \times \mathbf{r}_{3}=\mathbf{r}_{1}, \quad \mathbf{r}_{3} \times \mathbf{r}_{1}=\mathbf{r}_{2}, \quad \mathbf{r}_{1} \times \mathbf{r}_{2}=\mathbf{r}_{3}
$$

The $3 \times 3$ skew-symmetric matrix $\Omega$ can be written as $\Omega \boldsymbol{v}=\boldsymbol{\omega} \times \boldsymbol{v}$ for any vector $\boldsymbol{v} \in \mathbb{R}^{3}$ and $\boldsymbol{\omega} \in \mathbb{R}^{3}$, thus

$$
R \Omega R^{t}=R\left(\boldsymbol{\omega} \times \mathbf{r}_{1}\left|\boldsymbol{\omega} \times \mathbf{r}_{2}\right| \boldsymbol{\omega} \times \mathbf{r}_{3}\right)
$$




$$
=\left(\begin{array}{ccc}
0 & \mathbf{r}_{1} \cdot\left(\boldsymbol{\omega} \times \mathbf{r}_{2}\right) & \mathbf{r}_{1} \cdot\left(\boldsymbol{\omega} \times \mathbf{r}_{3}\right) \\
\mathbf{r}_{2} \cdot\left(\boldsymbol{\omega} \times \mathbf{r}_{1}\right) & 0 & \mathbf{r}_{2} \cdot\left(\boldsymbol{\omega} \times \mathbf{r}_{3}\right) \\
\mathbf{r}_{3} \cdot\left(\boldsymbol{\omega} \times \mathbf{r}_{1}\right) & \mathbf{r}_{3} \cdot\left(\boldsymbol{\omega} \times \mathbf{r}_{2}\right) & 0
\end{array}\right)
$$

Using (3.20) after rearranging cyclically, gives us

$$
R \Omega R^{t}=\left(\begin{array}{ccc}
0 & -\mathbf{r}_{3} \cdot \boldsymbol{\omega} & \mathbf{r}_{2} \cdot \boldsymbol{\omega} \\
\mathbf{r}_{3} \cdot \boldsymbol{\omega} & 0 & -\mathbf{r}_{1} \cdot \boldsymbol{\omega} \\
-\mathbf{r}_{2} \cdot \boldsymbol{\omega} & \mathbf{r}_{1} \cdot \boldsymbol{\omega} & 0
\end{array}\right)
$$

The last matrix is skew-symmetric which can be written in terms of the vector $\boldsymbol{\omega}$ as $R \boldsymbol{\omega}$ and that is the adjoint representation of $S O(3, \mathbb{R})$ on its Lie algebra. The adjoint representation of $S O(3, \mathbb{R})$ is the same as its defining representation on $\mathbb{R}^{3}$ so the standard representation coincides with the adjoint representation. This is an accidental property of three dimensions which does not generalise 47.

Theorem 3.4.1. (Kraft [31]) Adjoint representation of $S O(3, \mathbb{R})$ on $\mathfrak{s o}(3, \mathbb{R})$ is isomorphic to the natural representation of the same group on $\mathbb{R}^{3}$ where the isomorphism $\varphi: \mathfrak{s o}(3, \mathbb{R}) \longrightarrow \mathbb{R}^{3}$ given by

$$
\varphi(X)=\left(\begin{array}{c}
t_{1} \\
t_{2} \\
t_{3}
\end{array}\right) \in \mathbb{R}^{3}, \quad \text { where } X \in \mathfrak{s o}(3, \mathbb{R}) .
$$

With an easy calculation you can note that $\operatorname{tr} X^{2}=-2|\varphi(X)|^{2}$ and $X^{3}=-|\varphi(X)|^{2} X$. Moreover, $\varphi(X)^{t} X=0$. 
The adjoint action of $S E(3)$ on its Lie algebra is now simple to compute. For a typical Lie algebra element in $4 \times 4$ matrix form, we have

$$
\begin{aligned}
\left(\begin{array}{ll}
\Omega^{\prime} & \boldsymbol{v}^{\prime} \\
\mathbf{0} & 0
\end{array}\right) & =\left(\begin{array}{ll}
R & \mathbf{t} \\
\mathbf{0} & 1
\end{array}\right)\left(\begin{array}{cc}
\Omega & \boldsymbol{v} \\
\mathbf{0} & 0
\end{array}\right)\left(\begin{array}{cc}
R^{t} & -R^{t} \mathbf{t} \\
\mathbf{0} & 1
\end{array}\right) \\
& =\left(\begin{array}{cc}
R \Omega R^{t} & R \boldsymbol{v}-R \Omega R^{t} \mathbf{t} \\
\mathbf{0} & 0
\end{array}\right) .
\end{aligned}
$$

$R \Omega R^{t}$ is the adjoint action of $S O(3, \mathbb{R})$ and equal to $R \boldsymbol{\omega}$. However,

$$
-R \Omega R^{t} \mathbf{t}=-(R \boldsymbol{\omega}) \times \mathbf{t}=\mathbf{t} \times(R \boldsymbol{\omega}),
$$

and this can be written as $T R \boldsymbol{\omega}$. In six-component vector form of the Lie algebra, the representation has the form

$$
\left(\begin{array}{l}
\boldsymbol{\omega}^{\prime} \\
\boldsymbol{v}^{\prime}
\end{array}\right)=\left(\begin{array}{cc}
R & 0 \\
T R & R
\end{array}\right)\left(\begin{array}{l}
\boldsymbol{\omega} \\
\boldsymbol{v}
\end{array}\right)
$$

Summary, a rotation by $R \in S O(3, \mathbb{R})$ followed by a translation $\mathbf{t} \in \mathbb{R}^{3}$ is represented by the $6 \times 6$ matrix as follows:

$$
\left(\begin{array}{cc}
R & 0 \\
T R & R
\end{array}\right) .
$$

Definition 3.4.2. Adjoint action of the special Euclidean group is represented as

$$
\alpha_{2}: S E(3) \times \mathfrak{s e}(3) \longrightarrow \mathfrak{s e}(3)
$$




$$
\begin{aligned}
\alpha_{2}((R, \mathbf{t}),(\boldsymbol{\omega}, \boldsymbol{v})) & =\left(\begin{array}{cc}
R & 0 \\
T R & R
\end{array}\right)\left(\begin{array}{l}
\boldsymbol{\omega} \\
\boldsymbol{v}
\end{array}\right) \\
& =\left(\begin{array}{c}
R \boldsymbol{\omega} \\
T R \boldsymbol{\omega}+R \boldsymbol{v}
\end{array}\right)
\end{aligned}
$$

where $R \in S O(3, \mathbb{R}), \mathbf{t} \in \mathbb{R}^{3}$, and $T \in \mathfrak{s o}(3, \mathbb{R})$.

\subsection{Twists and Plücker coordinates}

As a special case when $n=3$, the Lie algebra $\mathfrak{s e}(3)$ elements are called Ball's screws or twists and hence

$$
\mathfrak{s e}(3, \mathbb{R})=\left\{(B, \mathbf{b}) \quad \mid \quad B \in \mathfrak{s o}(3, \mathbb{R}), \mathbf{b} \in \mathbb{R}^{3}\right\}
$$

A $3 \times 3$ skew-symmetric matrix $B$ has only three independent elements. Those elements can be assembled into the vector $\boldsymbol{\omega}$. The action of the skew symmetric matrix $B$ on an arbitrary vector $\mathbf{y}$ is equivalent to the cross product by $\boldsymbol{\omega}$, that is $B \mathbf{y}=\boldsymbol{\omega} \times \mathbf{y}$. Hence, we can write any element in $\mathfrak{s e}(3, \mathbb{R})$, using the six-dimensional vector $\left(w_{1}, w_{2}, w_{3}, v_{1}, v_{2}, v_{3}\right)=(\boldsymbol{\omega}, \boldsymbol{v})$ the Plücker coordinates of the twist where $\boldsymbol{v}=\mathbf{b} 35,47]$.

We should write $\left(\boldsymbol{\omega}^{t}, \boldsymbol{v}^{t}\right)^{t}$ but where there is no likehood of confusion we simply write $(\boldsymbol{\omega}, \boldsymbol{v})$ for the Plücker coordinates of a twist. The term screw is frequently used in place of twist but properly a screw is a projective twist (i.e. only defined up to non-zero multiple).

Twist and screws are fundamental concepts in Kinematics, and the general theory is developed comprehensively in Ball [2], and Hunt [25].

Ball defined a twist as follows: A body is said to receive a twist about 
a screw when it is rotated uniformly about the screw, while it is translated uniformly parallel to screw through a distance equal to the product of the pitch and the circular measure of the angle of rotation. A screw is a straight line with which a definite linear magnitude termed in the pitch, is associated. The pitch is the rectilinear distance through which the body is translated parallel to the axis of the screw, while the body is rotated through the angular unit of circular measure and it is equal the ratio of the translational displacement and the rotational displacement of the body given as:

$$
h=\frac{\omega \cdot \boldsymbol{v}}{\omega \cdot \omega} .
$$

The vector $\boldsymbol{v}$ of the screw $(\boldsymbol{\omega}, \boldsymbol{v})$ can be decomposed into components parallel to and perpendicular to $\boldsymbol{\omega}$, see Figure (3.5). The parallel component is the velocity along the axis $\boldsymbol{v}_{\|}=h \boldsymbol{\omega}$ where $h$ is the pitch of the screw and given in 3.29$)$. The perpendicular component is the moment of the axis about the origin $\boldsymbol{v}_{\perp}=\boldsymbol{v}-h \boldsymbol{\omega}$, so we can determine a vector $\mathbf{q}$ such that this vector satisfies the equation

$$
\mathbf{q} \times \boldsymbol{\omega}=\boldsymbol{v}-h \boldsymbol{\omega} .
$$

The solution of this equation is obtained by computing the cross product of both sides with $\boldsymbol{\omega}$, in which case we obtain

$$
\mathrm{q}=\frac{\omega \times v}{\omega \cdot \omega}
$$

If the screw takes the form $(\boldsymbol{\omega}, h \boldsymbol{\omega})$ with its linear and angular velocity vectors aligned in the direction vector $\boldsymbol{\omega}$, then in this case we can see explicitly 


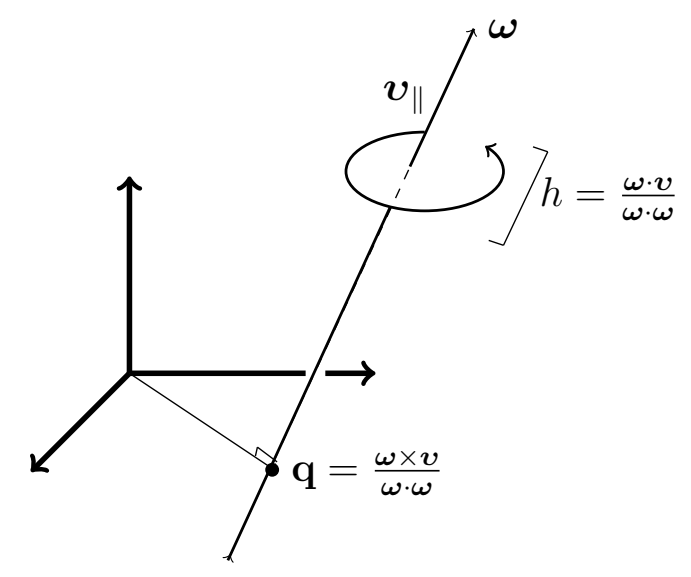

Figure 3.1: A screw line in three dimensional space, where $\boldsymbol{v}$ has been decomposed into parallel and perpendicular to $\boldsymbol{\omega}$, and $h$ is its pitch.

that the body moves in screw motion. The points in a body undergoing a constant screw motion trace helices in the fixed frame. If $\boldsymbol{v}=0$, implies to screw motion has zero pitch, then the trajectories of points are circles, and the movement is a pure rotation. If $\boldsymbol{\omega}=0$ implies to screw motion has infinite pitch, then the trajectories are all straight lines in the same direction.

The Lie bracket of two screws given by Plücker coordinates $S_{1}=\left(\boldsymbol{\omega}_{1}, \boldsymbol{v}_{1}\right)$ and $S_{2}=\left(\boldsymbol{\omega}_{2}, \boldsymbol{v}_{2}\right)$ is

$$
\left[S_{1}, S_{2}\right]=\left(\boldsymbol{\omega}_{1} \times \boldsymbol{\omega}_{2}, \boldsymbol{\omega}_{1} \times \boldsymbol{v}_{2}+\boldsymbol{v}_{1} \times \boldsymbol{\omega}_{2}\right)
$$

In Chapter 8 we shall talk more about screws and their application in Denavit-Hartenberg parameters of robot arms. However, in Chapter 9 we shall introduce the physical meaning of the Lie bracket of two screws with more details in geometrical duality of robot arms. 


\section{Chapter 4}

\section{Invariant Theory of $S O(3, \mathbb{R})$}

and $S E(3)$

\subsection{Polynomial rings and rings of invariants}

Definition 4.1.1. (Kraft [31], Neusel [37]). Let $\rho: G \longrightarrow G L(n, \mathbb{k})$ be a group representation, and $\mathbb{k}[\mathbf{V}]=\mathbb{k}\left[x_{1}, \cdots, x_{n}\right]$ where $\mathbf{V}=\mathbb{k}^{n}$ be the ring of polynomials in $n$ indeterminates $x_{1}, \cdots, x_{n}$ with coefficients from a field $\mathbb{k}$, then a polynomial $f \in \mathbb{k}\left[x_{1}, \cdots, x_{n}\right]$ is invariant under the group action of $G$ if

$$
f(\mathbf{x})=f(A \mathbf{x}), \quad \text { where } \mathbf{x}=\left(x_{1}, \cdots, x_{n}\right),
$$

for all $A \in G$. The subset of all invariant polynomials is denoted $\mathbb{k}\left[x_{1}, \cdots, x_{n}\right]^{G}$.

In Definition 4.1.1 for a non matrix group we denote $A \mathbf{x}=\rho(A) \mathbf{x}$ since not all groups are matrix groups. Moreover, note that this is a somewhat sloppy notation since the $f^{\prime} s$ are invariant under representation $\rho(G)$. Since 
the same group can have various representations we should write $\mathbb{k}[\mathbf{V}]^{\rho(G)}$.

Proposition 4.1.2. The set $\mathbb{k}[\mathbf{V}]^{G} \subseteq \mathbb{k}[\mathbf{V}]$ of invariant polynomials forms an $\mathbb{k}$-subalgebra.

Proof. See 37] page 55.

$\mathbb{k}[\mathbf{V}]^{G}$ is a commutative integral domain, because it is the subring of the commutative integral domain $\mathbb{k}[\mathbf{V}]$. Furthermore, the ring of polynomial invariants inherits the grading from $\mathbb{k}[\mathbf{V}]$, because the group action respects homogeneity.

Example 4.1.3. Let $G=S O(3, \mathbb{R})$ acting on $\mathbb{R}^{3}$ by $f(\mathbf{x})=x_{1}^{2}+x_{2}^{2}+x_{3}^{2}=\mathbf{x} \cdot \mathbf{x}$ $f$ is invariant under $G$ since

$$
f(A \mathbf{x})=A \mathbf{x} \cdot A \mathbf{x}=\mathbf{x} \cdot \mathbf{x}=f(\mathbf{x})
$$

for all $A \in S O(3, \mathbb{R})$.

Example 4.1.4. The Klein form $\boldsymbol{\omega} \cdot \boldsymbol{v}$ and Killing form $\boldsymbol{\omega} \cdot \boldsymbol{\omega}$ are real invariants of the adjoint action of the special Euclidean group where $(\boldsymbol{v}, \boldsymbol{\omega})$ are Plücker coordinates for $\mathfrak{s e}(3)$ 47]. They can be expressed as a polynomial where

$$
\begin{aligned}
& f(\boldsymbol{\omega}, \boldsymbol{v})=\boldsymbol{\omega} \cdot \boldsymbol{\omega}=w_{1}^{2}+w_{2}^{2}+w_{3}^{2}, \\
& g(\boldsymbol{\omega}, \boldsymbol{v})=\boldsymbol{\omega} \cdot \boldsymbol{v}=w_{1} v_{1}+w_{2} v_{2}+w_{3} v_{3} .
\end{aligned}
$$

Let $(R, \mathbf{t})$ be an element in $S E(3)$, and $\rho((R, \mathbf{t}))=A$ be the representation of the adjoint action of $S E(3)$ defined in Theorem 3.4.2. We can show 
$f$ and $g$ are invariant under the adjoint action of $S E(3)$. Firstly,

$$
\begin{aligned}
f(A(\boldsymbol{\omega}, \boldsymbol{v})) & =\left(\left(\begin{array}{cc}
R & 0 \\
T R & R
\end{array}\right)\left(\begin{array}{l}
\boldsymbol{\omega} \\
\boldsymbol{v}
\end{array}\right)\right) \\
& =f(R \boldsymbol{\omega}, T R \boldsymbol{\omega}+R \boldsymbol{v})=R \boldsymbol{\omega} \cdot R \boldsymbol{\omega} \\
& =\boldsymbol{\omega} \cdot \boldsymbol{\omega}=f(\boldsymbol{\omega}, \boldsymbol{v}) .
\end{aligned}
$$

Secondly,

$$
g(A(\boldsymbol{\omega}, \boldsymbol{v}))=g(R \boldsymbol{\omega}, T R \boldsymbol{\omega}+R \boldsymbol{v})=(R \boldsymbol{\omega}) \cdot(T R \boldsymbol{\omega}+R v)
$$

Since $T$ is skew-symmetric matrix then $T R \boldsymbol{\omega}=\mathbf{t} \times R \boldsymbol{\omega}$, thus

$$
\begin{aligned}
& =(R \boldsymbol{\omega}) \cdot(\mathbf{t} \times R \boldsymbol{\omega})+R \boldsymbol{\omega} \cdot R \boldsymbol{v}=\mathbf{t} \cdot(\underbrace{R \boldsymbol{\omega} \times R \boldsymbol{\omega}}_{\text {zero }})+\boldsymbol{\omega} \cdot \boldsymbol{v} \\
& =\boldsymbol{\omega} \cdot \boldsymbol{v}=g(\boldsymbol{\omega}, \boldsymbol{v}) .
\end{aligned}
$$

\subsection{Vector invariants}

Lemma 4.2.1. (Neusel [37]). The representation $\rho: G \longrightarrow G L(n, \mathbb{k})$ is faithful if and only if the induced group action of $G$ on $\mathbb{k}^{n}$ is faithful.

Proof. See Lemma 3.7 in [37].

Definition 4.2.2. (Neusel 37]). Let $\rho: G \longrightarrow G L(n, \mathbb{k})$ be a faithful group representation. Set $\rho_{1}=\rho$ and define a new representation

$$
\rho_{2}: G \longrightarrow G L(2 n, \mathbb{k})
$$


afforded by the block matrices

$$
\rho_{2}(g)=\left(\begin{array}{cc}
\rho_{1}(g) & 0 \\
0 & \rho_{1}(g)
\end{array}\right) .
$$

Iteratively we define

$$
\rho_{k}: G \longrightarrow G L(k n, \mathbb{k}), \quad g \mapsto \operatorname{diagonal}\left(\rho_{1}(g), \cdots, \rho_{1}(g)\right)
$$

We say that $\rho_{k}$ is the $k$-fold vector representation of $\rho$. The group $G$ acts via $\rho_{k}$ by acting on the $k$ vectors simultaneously. The corresponding ring of invariants is called the ring of vector invariants [37].

\subsection{The first fundamental theorem of invari- ant theory for $S O(3, \mathbb{R})$}

The first fundamental theorem for the special orthogonal group $S O(3, \mathbb{R})$ was proved by Weyl [49] and he stated the vector invariants of the orthogonal group acting on a standard action on $\mathbb{R}^{n}$. For three dimensions, the standard action of the orthogonal group is isomorphic to the adjoint action of the same group. Weyl divided the vector invariants of the orthogonal group into odd and even invariants. Moreover, the fundamental theorem of invariant theory of $S O(3, \mathbb{R})$ consists of all those odd and even vector invariants.

Theorem 4.3.1. For the $m$-fold vector representation of $S O(3, \mathbb{R})$

(a) Every even invariant can be written as a polynomial in the scalar products

$$
\omega_{i} \cdot \omega_{j}
$$


for $1 \leq i \leq j \leq m$, where $\boldsymbol{\omega}_{i}, \boldsymbol{\omega}_{j} \in \mathbb{R}^{3}$.

(b) Every odd invariant is a sum of terms of the form

$$
\left[\begin{array}{lll}
\boldsymbol{\omega}_{i} & \boldsymbol{\omega}_{j} & \boldsymbol{\omega}_{k}
\end{array}\right] f^{*}\left(\boldsymbol{\omega}_{1}, \cdots, \boldsymbol{\omega}_{m}\right)
$$

for $1 \leq i \leq j \leq k \leq m$, where $\boldsymbol{\omega}_{i}, \boldsymbol{\omega}_{j}, \boldsymbol{\omega}_{k} \in \mathbb{R}^{3}$, and $f^{*}$ is an even invariant. Square bracket means the determinant of those three vectors.

Proof. See Theorem 2.9.A in 49 .

Corollary 4.3.2. The $m$-fold vector invariants of $S O(3, \mathbb{R})$ are generated by

(a) for $m=1$ :

$$
\boldsymbol{\omega}_{1} \cdot \boldsymbol{\omega}_{1}
$$

(b) for $m=2$ :

$$
\boldsymbol{\omega}_{1} \cdot \boldsymbol{\omega}_{1}, \quad \boldsymbol{\omega}_{1} \cdot \boldsymbol{\omega}_{2}, \quad \boldsymbol{\omega}_{2} \cdot \boldsymbol{\omega}_{2}
$$

(c) for $m=3$ :

$$
\begin{gathered}
\boldsymbol{\omega}_{1} \cdot \boldsymbol{\omega}_{1}, \quad \boldsymbol{\omega}_{1} \cdot \boldsymbol{\omega}_{2}, \quad \boldsymbol{\omega}_{1} \cdot \boldsymbol{\omega}_{3}, \quad \boldsymbol{\omega}_{2} \cdot \boldsymbol{\omega}_{2}, \quad \boldsymbol{\omega}_{2} \cdot \boldsymbol{\omega}_{3}, \quad \boldsymbol{\omega}_{3} \cdot \boldsymbol{\omega}_{3}, \\
{\left[\begin{array}{lll}
\boldsymbol{\omega}_{1} & \boldsymbol{\omega}_{2} & \boldsymbol{\omega}_{3}
\end{array}\right]=\left|\begin{array}{lll}
\boldsymbol{\omega}_{1} & \boldsymbol{\omega}_{2} & \boldsymbol{\omega}_{3}
\end{array}\right|=\boldsymbol{\omega}_{1} \cdot\left(\boldsymbol{\omega}_{2} \times \boldsymbol{\omega}_{3}\right) .}
\end{gathered}
$$

Proof. This follows directly from Theorem 4.3.1. 


\subsection{The second fundamental theorem of in- variant theory for $S O(3, \mathbb{R})$}

The second fundamental theorem of invariants is about the algebraic relations among these invariants which are also called syzygies. In Weyl [49], the syzygies of $S O(n, \mathbb{R})$ were fully described. We are interested in algebraic relations of $S O(3, \mathbb{R})$ acting on vectors. However, there are no relations when there are at most two vectors, the syzygies start when there are at least three vectors and hence the syzygies of $m$-fold vector representation of $S O(3, \mathbb{R}):$

(a) acting on three vectors $\boldsymbol{\omega}_{1}, \boldsymbol{\omega}_{2}, \boldsymbol{\omega}_{3} \in \mathbb{R}^{3}$ is

$$
\left[\begin{array}{lll}
\boldsymbol{\omega}_{1} & \boldsymbol{\omega}_{2} & \boldsymbol{\omega}_{3}
\end{array}\right]^{2}-\left|\begin{array}{lll}
\boldsymbol{\omega}_{1} \cdot \boldsymbol{\omega}_{1} & \boldsymbol{\omega}_{1} \cdot \boldsymbol{\omega}_{2} & \boldsymbol{\omega}_{1} \cdot \boldsymbol{\omega}_{3} \\
\boldsymbol{\omega}_{2} \cdot \boldsymbol{\omega}_{1} & \boldsymbol{\omega}_{2} \cdot \boldsymbol{\omega}_{2} & \boldsymbol{\omega}_{2} \cdot \boldsymbol{\omega}_{3} \\
\boldsymbol{\omega}_{3} \cdot \boldsymbol{\omega}_{1} & \boldsymbol{\omega}_{3} \cdot \boldsymbol{\omega}_{2} & \boldsymbol{\omega}_{3} \cdot \boldsymbol{\omega}_{3}
\end{array}\right|=0
$$

(b) acting on four vectors $\boldsymbol{\omega}_{1}, \boldsymbol{\omega}_{2}, \boldsymbol{\omega}_{3}, \boldsymbol{\omega}_{4} \in \mathbb{R}^{3}$ are the first type syzygy in (4.3) and this type

$$
\sum_{\boldsymbol{\omega}_{1} \cdots \boldsymbol{\omega}_{4}} \pm\left[\begin{array}{lll}
\boldsymbol{\omega}_{1} & \boldsymbol{\omega}_{2} & \boldsymbol{\omega}_{3}
\end{array}\right]\left(\boldsymbol{\omega}_{4} \cdot \boldsymbol{\omega}\right)=0
$$

for any $\boldsymbol{\omega} \in \mathbb{R}^{3}$.

(c) acting on $n$-fold vectors in $\mathbb{R}^{3}$ are the first type syzygy 4.3 and this type

$$
\sum_{\lambda=1}^{n}(-1)^{\lambda-1}\left[\boldsymbol{\omega}_{i_{1}} \cdots \boldsymbol{\omega}_{i_{\lambda-1}} \boldsymbol{\omega}_{i_{\lambda+1}} \cdots \boldsymbol{\omega}_{i_{k}}\right]\left(\boldsymbol{\omega}_{i_{\lambda}} \cdot \boldsymbol{\omega}_{j}\right)=0
$$


for any $\boldsymbol{\omega}_{j}$ and $\quad 1 \leq i_{1} \leq \cdots \leq i_{\lambda} \leq \cdots \leq i_{k} \leq n$.

\subsection{The first fundamental theorem of invari- ant theory for $S E(3)$}

Theorem 4.5.1. (Donelan et al [16]). Every polynomial invariant of the adjoint action of $S E(3)$ belongs to $\mathbb{R}[\boldsymbol{\omega} \cdot \boldsymbol{\omega}, \boldsymbol{\omega} \cdot \boldsymbol{v}]$.

Proof. See Theorem 3.1 in $[16]$.

Theorem 4.5.2. The invariant ring of $S E(3)$ acting on two screws is generated by $\boldsymbol{\omega}_{i} \cdot \boldsymbol{\omega}_{j}, \boldsymbol{\omega}_{i} \cdot \boldsymbol{v}_{i}, \boldsymbol{\omega}_{1} \cdot \boldsymbol{v}_{2}+\boldsymbol{v}_{1} \cdot \boldsymbol{\omega}_{2}($ for $1 \leq i \leq j \leq 2)$.

Proof. See Theorem 7.4.1 in 14, 39]. 


\section{Chapter 5}

\section{Algebraic Mappings Among $S O(3, \mathbb{R}), S O(3, \mathbb{D})$ and $S E(3)$}

\subsection{Preliminaries}

Lemma 5.1.1. If $f$ be any $S O(3, \mathbb{R})$ invariant polynomial, then given $\boldsymbol{\omega} \in$ $\mathbb{R}^{3}$ there is a scalar $\lambda$ such that $\nabla f(\boldsymbol{\omega})=\lambda \boldsymbol{\omega}^{t}$.

Proof. Suppose $f$ is $S O(3, \mathbb{R})$ invariant polynomial defined as $f: \mathbb{R}^{3} \longrightarrow \mathbb{R}$. Choose $A \in S O(3, \mathbb{R})$, then for any $\boldsymbol{\omega} \in \mathbb{R}^{3}$ we have $f(A \boldsymbol{\omega})=f(\boldsymbol{\omega})$ since $f$ is $S O(3, \mathbb{R})$ invariant. In addition $\|A \boldsymbol{\omega}\|=\|\boldsymbol{\omega}\|$ so they are contained in the same sphere. This means that the level sets of $f$ are unions of spheres (except for the origin $\mathbf{0}$ ). From vector calculus $\nabla f$ is orthogonal to the level surface of $f$. Moreover $\nabla f$ is parallel to $\boldsymbol{\omega}^{t}$ since the last one is also orthogonal to the sphere, therefore there is a scalar $\lambda$ such that

$$
\nabla f(\boldsymbol{\omega})=\lambda \boldsymbol{\omega}^{t}
$$


Theorem 5.1.2. There is a Lie group isomorphism between the dual special orthogonal group and the special Euclidean group given by :

$$
\begin{aligned}
\phi: S O(3, \mathbb{D}) & \longrightarrow S E(3) \\
\phi\left(A_{0}+\epsilon A_{1}\right) & =\left(\begin{array}{ll}
A_{0} & 0 \\
A_{1} & A_{0}
\end{array}\right)_{6 \times 6}
\end{aligned}
$$

Proof. We show that $\phi$ is a homomorphism, bijective. To prove $\phi$ is a homomorphism, let $\hat{A}, \hat{B} \in S O(3, \mathbb{D})$, then

$$
\begin{aligned}
\phi(\hat{A} \hat{B}) & =\phi\left(\left(A_{0}+\epsilon A_{1}\right)\left(B_{0}+\epsilon B_{1}\right)\right) \\
& =\phi\left(A_{0} B_{0}+\epsilon\left(A_{0} B_{1}+A_{1} B_{0}\right)\right) \\
& =\left(\begin{array}{cc}
A_{0} B_{0} & 0 \\
A_{0} B_{1}+A_{1} B_{0} & A_{0} B_{0}
\end{array}\right) \\
& =\left(\begin{array}{cc}
A_{0} & 0 \\
A_{1} & A_{0}
\end{array}\right)\left(\begin{array}{cc}
B_{0} & 0 \\
B_{1} & B_{0}
\end{array}\right) \\
& =\phi(\hat{A}) \phi(\hat{B}) .
\end{aligned}
$$

To show $\phi$ is injective, assume $\phi\left(A_{0}+\epsilon A_{1}\right)=\phi\left(B_{0}+\epsilon B_{1}\right)$, then $\phi$ map above obtains this equality:

$$
\left(\begin{array}{cc}
A_{0} & 0 \\
A_{1} & A_{0}
\end{array}\right)=\left(\begin{array}{cc}
B_{0} & 0 \\
B_{1} & B_{0}
\end{array}\right) .
$$

This implies to $A_{0}=B_{0}, A_{1}=B_{1}$, therefore

$$
A_{0}+\epsilon A_{1}=B_{0}+\epsilon B_{1}
$$


To show that $\phi$ is onto. It is clear that for all $x \in S E(3)$ there exists $y \in S O(3, \mathbb{D})$ such that $\phi(y)=x$.

The inverse for the map $\phi$ exists and defined by:

$$
\begin{aligned}
\varphi: S E(3) & \longrightarrow S O(3, \mathbb{D}) \\
\varphi\left(\left(\begin{array}{cc}
A_{0} & 0 \\
A_{1} & A_{0}
\end{array}\right)\right) & =A_{0}+\epsilon A_{1} .
\end{aligned}
$$

This map is well-defined. It is enough to prove $\varphi$ is bijective, and the proof is similar to $\phi$.

Proposition 5.1.3. In Theorem 5.1.2 we used $6 \times 6$-matrix representation $\left(\begin{array}{cc}A_{0} & 0 \\ A_{1} & A_{0}\end{array}\right)$ to represent the adjoint action of $S E(3)$, and in Theorem 3.4.2 we used different $6 \times 6$-matrix representation $\left(\begin{array}{cc}R & 0 \\ T R & R\end{array}\right)$ to represent the same group under the same action. However, both representations are the same, where $A_{0}=R$ and $T=A_{1} A_{0}^{t}$.

Lemma 5.1.4. There is a Lie group isomorphism between the dual special orthogonal group acting on dual vector and the special Euclidean group acting on single screw given by

$$
\begin{array}{r}
\phi_{1}: S O(3, \mathbb{D}) \times \mathfrak{D}^{3} \longrightarrow S E(3) \times \mathfrak{s e}(3) \\
\phi_{1}(\hat{A}, \hat{\mathbf{u}})=\left(\left(A_{0}, A_{1} A_{0}^{-1}\right),(\boldsymbol{\omega}, \boldsymbol{v})\right),
\end{array}
$$

where $\hat{A}=A_{0}+\epsilon A_{1} \in S O(3, \mathbb{D})$ and $\hat{\mathbf{u}}=\boldsymbol{\omega}+\epsilon \boldsymbol{v} \in \mathfrak{D}^{3}$

Definition 5.1.5. There is Lie algebra isomorphism between the 3dimensional dual Lie algebra and special Euclidean Lie algebra represented 
by using Plücker coordinates :

$$
\begin{aligned}
& \psi: \mathfrak{D}^{3} \longrightarrow \mathfrak{s e}(3) \\
& \psi(\boldsymbol{\omega}+\epsilon \boldsymbol{v})=\left(\begin{array}{l}
\boldsymbol{\omega} \\
\boldsymbol{v}
\end{array}\right) .
\end{aligned}
$$

Definition 5.1.6. Adjoint action of the dual special orthogonal group is represented as

$$
\begin{aligned}
& \alpha_{1}: S O(3, \mathbb{D}) \times \mathfrak{D}^{3} \longrightarrow \mathfrak{D}^{3} \\
& \alpha_{1}(\hat{A}, \hat{\mathbf{u}})=\hat{A} \hat{\mathbf{u}}
\end{aligned}
$$

where $\hat{A} \in S O(3, \mathbb{D})$ and $\hat{\mathbf{u}} \in \mathfrak{D}^{3}$.

Theorem 5.1.7. This diagram is commutative

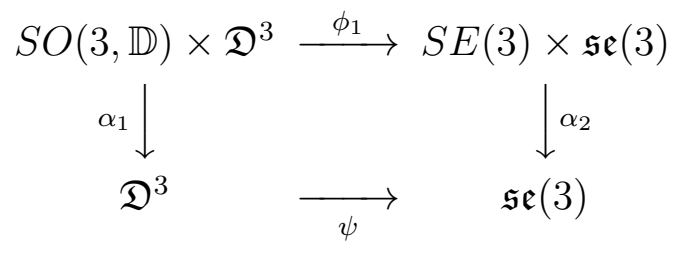

Proof. We must show that $\psi \circ \alpha_{1}=\alpha_{2} \circ \phi_{1}$. Assume $\hat{A} \in S O(3, \mathbb{D})$, so we can write $\hat{A}=A_{0}+\epsilon A_{1}$. Suppose $\hat{\mathbf{u}} \in \mathbb{D}^{3}$, then we can write $\hat{\mathbf{u}}=\boldsymbol{\omega}+\epsilon \boldsymbol{v}$ where $\boldsymbol{\omega}, \boldsymbol{v} \in \mathbb{R}^{3}$. Firstly, by using Definition 5.1 .6

$$
\begin{aligned}
\alpha_{1}(\hat{A}, \hat{\mathbf{u}}) & =\hat{A} \hat{\mathbf{u}} \\
& =\underbrace{A_{0} \boldsymbol{\omega}}_{\mathbb{R}^{3}}+\epsilon \underbrace{\left(A_{1} \boldsymbol{\omega}+A_{0} \boldsymbol{v}\right)}_{\mathbb{R}^{3}} \in \mathbb{D}^{3} \\
\left(\psi \circ \alpha_{1}\right)(\hat{A} \hat{\mathbf{u}}) & =\psi\left(\alpha_{1}(\hat{A} \hat{\mathbf{u}})\right)=\psi\left(A_{0} \boldsymbol{\omega}+\epsilon\left(A_{1} \boldsymbol{\omega}+A_{0} \boldsymbol{v}\right)\right)
\end{aligned}
$$




$$
=\left(\begin{array}{c}
A_{0} \boldsymbol{\omega} \\
A_{1} \boldsymbol{\omega}+A_{0} \boldsymbol{v}
\end{array}\right) .
$$

Secondly, by using Definition 3.4 .2

$$
\begin{aligned}
\phi_{1}(\hat{A}, \hat{\mathbf{u}}) & =\left(\left(A_{0}, A_{1} A_{0}^{-1}\right),(\boldsymbol{\omega}, \boldsymbol{v})\right) \\
\alpha_{2} \circ \phi_{1} & =\alpha_{2}\left(\phi(\hat{A} \hat{\mathbf{u}})=\alpha_{2}\left(\left(A_{0}, A_{1} A_{0}^{-1}\right),(\boldsymbol{\omega}, \boldsymbol{v})\right)\right. \\
& =\left(\begin{array}{cc}
A_{0} & 0 \\
A_{1} A_{0}^{-1} A_{0} & A_{0}
\end{array}\right)\left(\begin{array}{l}
\boldsymbol{\omega} \\
\boldsymbol{v}
\end{array}\right) \\
& =\left(\begin{array}{c}
A_{0} \boldsymbol{\omega} \\
A_{1} \boldsymbol{\omega}+A_{0} \boldsymbol{v}
\end{array}\right) .
\end{aligned}
$$

Since (5.6) and (5.7) are equal, the diagram commutes.

This tells us that the adjoint action of dual orthogonal group is essentially the same as (isomorphic to) that for the Euclidean group.

\subsection{The algebraic structures on the dual mapping}

This part introduces the algebraic structures on which the dual mapping operates. Duffy and Rico [17] studied the commutative algebra over the real field, consisting of real differentiable functions defined on the ring of polynomials $\mathbb{R}[\mathbf{x}]$, similarly consider the commutative algebra over the real field of continuous functions defined over suitable $\mathbf{x} \times \mathbf{y} \subset \mathbb{R}^{n} \times \mathbb{R}^{n}$ with coefficients over the dual numbers denoted $\mathbb{D}[\mathbf{x} \times \mathbf{y}]$. 
Definition 5.2.1. (Duffy and Rico $[17]$ ). The dual mapping $\boldsymbol{\delta}: \mathbb{R}[\mathbf{x}] \longrightarrow$ $\mathbb{D}[\mathbf{x} \times \mathbf{y}]$ is defined as:

$$
\begin{aligned}
\boldsymbol{\delta}\left[f\left(x_{1}, x_{2}, \cdots, x_{n}\right)\right] & =\hat{f}\left(x_{1}, x_{2}, \cdots, x_{n} ; y_{1}, y_{2}, \cdots, y_{n}\right) \\
& =f\left(x_{1}, x_{2}, \cdots, x_{n}\right)+\epsilon \sum_{r=1}^{n} y_{r} \frac{\partial f}{\partial x_{r}}(\mathbf{x}) \\
& =f(\mathbf{x})+\epsilon \nabla f(\mathbf{x}) \mathbf{y}, \quad \text { where } \epsilon^{2}=0 .
\end{aligned}
$$

Theorem 5.2.2. (Duffy and Rico [17]). The dual mapping $\boldsymbol{\delta}: \mathbb{R}[\mathbf{x}] \longrightarrow$ $\mathbb{D}(\mathrm{x} \times \mathrm{y})$ is an algebra homomorphism.

Proof. We must show that :

(a) $\boldsymbol{\delta}[g+h]=\boldsymbol{\delta}[g]+\boldsymbol{\delta}[h]$.

(b) $\boldsymbol{\delta}[g h]=\boldsymbol{\delta}[g] \boldsymbol{\delta}[h]$.

(c) $\boldsymbol{\delta}[\lambda g]=\lambda \boldsymbol{\delta}[g], \quad$ where $\lambda \in \mathbb{R}$.

Let $g\left(x_{1}, x_{2}, \cdots, x_{n}\right), h\left(x_{1}, x_{2}, \cdots, x_{n}\right) \in \mathbb{R}[\mathbf{x}]$, proving (a) and (c) are straightforward. We show (b):

(b) $\boldsymbol{\delta}[g(\mathbf{x}) h(\mathbf{x})]=\boldsymbol{\delta}\left[g\left(x_{1}, x_{2}, \cdots, x_{n}\right) h\left(x_{1}, x_{2}, \cdots, x_{n}\right)\right]$

$$
\begin{aligned}
& =g\left(x_{1}, x_{2}, \cdots, x_{n}\right) h\left(x_{1}, x_{2}, \cdots, x_{n}\right)+\epsilon \sum_{r=1}^{n} y_{r} \frac{\partial(g h)}{\partial x_{r}} \\
& =g\left(x_{1}, x_{2}, \cdots, x_{n}\right) h\left(x_{1}, x_{2}, \cdots, x_{n}\right)+\epsilon \sum_{r=1}^{n} y_{r}\left(h \frac{\partial g}{\partial x_{r}}+g \frac{\partial h}{\partial x_{r}}\right) \\
& =\left[g\left(x_{1}, x_{2}, \cdots, x_{n}\right)+\epsilon \sum_{r=1}^{n} y_{r} \frac{\partial g}{\partial x_{r}}\right]\left[h\left(x_{1}, x_{2}, \cdots, x_{n}\right)+\epsilon \sum_{r=1}^{n} y_{r} \frac{\partial h}{\partial x_{r}}\right] \\
& =\boldsymbol{\delta}\left[g\left(x_{1}, x_{2}, \cdots, x_{n}\right)\right] \boldsymbol{\delta}\left[h\left(x_{1}, x_{2}, \cdots, x_{n}\right)\right] \\
& =\boldsymbol{\delta}[g(\mathbf{x})] \boldsymbol{\delta}[h(\mathbf{x})] .
\end{aligned}
$$




\subsection{Dual invariants and the special orthogo- nal group}

Lemma 5.3.1. Let $f \in \mathbb{R}[\boldsymbol{\omega}]^{S O(3, \mathbb{R})}$, then $\hat{f} \in \mathbb{R}[\boldsymbol{\omega}, \boldsymbol{v}]$ is a dual vector invariant of $S O(3, \mathbb{D})$ i.e $\hat{f} \in \mathbb{R}[\boldsymbol{\omega}, \boldsymbol{v}]^{S O(3, \mathbb{D})}$.

Proof. We must show that $\hat{f}(\hat{A} \hat{\mathbf{u}})=\hat{f}(\hat{\mathbf{u}})$ for all $\hat{A} \in S O(3, \mathbb{D})$, and $\hat{\mathbf{u}} \in \mathbb{D}^{3}$. Let $\hat{A} \in S O(3, \mathbb{D})$ be defined as in Definition 2.4.3, and let $\hat{\mathbf{u}}=\boldsymbol{\omega}+\varepsilon \boldsymbol{v}$ where $\boldsymbol{\omega}, \boldsymbol{v} \in \mathbb{R}^{3}$. In addition $\hat{A} \hat{\mathbf{u}}=\left(A_{0}+\epsilon A_{1}\right)(\boldsymbol{\omega}+\epsilon \boldsymbol{v})=A_{0} \boldsymbol{\omega}+\epsilon\left(A_{1} \boldsymbol{\omega}+A_{0} \boldsymbol{v}\right)$.

$$
\begin{aligned}
\hat{f}(\hat{A} \hat{\mathbf{u}}) & =\hat{f}\left(A_{0} \boldsymbol{\omega} ; A_{1} \boldsymbol{\omega}+A_{0} \boldsymbol{v}\right), \quad \text { by Definition 5.2.1 we get } \\
& =f\left(A_{0} \boldsymbol{\omega}\right)+\epsilon \nabla f\left(A_{0} \boldsymbol{\omega}\right)\left(A_{1} \boldsymbol{\omega}+A_{0} \boldsymbol{v}\right)
\end{aligned}
$$

Since $f$ is an $S O(3, \mathbb{R})$ invariant then $f\left(A_{0} \boldsymbol{\omega}\right)=f(\boldsymbol{\omega})$. Moreover, using Lemma 5.1.1 we get,

$$
\begin{aligned}
\hat{f}(\hat{A} \hat{\mathbf{u}}) & =f(\boldsymbol{\omega})+\epsilon \nabla f(\boldsymbol{\omega}) A_{0}^{t}\left(A_{1} \boldsymbol{\omega}+A_{0} \boldsymbol{v}\right) \\
& =f(\boldsymbol{\omega})+\epsilon \nabla f(\boldsymbol{\omega}) A_{0}^{t} A_{1} \boldsymbol{\omega}+\epsilon \nabla f(\boldsymbol{\omega}) A_{0}^{t} A_{0} \boldsymbol{v} \\
& =f(\boldsymbol{\omega})+\epsilon \lambda \boldsymbol{\omega}^{t}\left(A_{0}^{t} A_{1}\right) \boldsymbol{\omega}+\epsilon \nabla f(\boldsymbol{\omega}) \boldsymbol{v}
\end{aligned}
$$

From Theorem 2.4.4 $A_{0}^{t} A_{1}$ is skew symmetric matrix and from Lemma 2.2.1 $\boldsymbol{\omega}^{t}\left(A_{0}^{t} A_{1}\right) \boldsymbol{\omega}=0$, and hence

$$
\begin{aligned}
\hat{f}(\hat{A} \hat{\mathbf{u}}) & =f(\boldsymbol{\omega})+\epsilon \nabla f(\boldsymbol{\omega}) \boldsymbol{v} \\
& =\hat{f}(\boldsymbol{\omega} ; \boldsymbol{v}) \\
& =\hat{f}(\hat{\mathbf{u}})
\end{aligned}
$$

Therefore, $\hat{f}$ is an invariant for $S O(3, \mathbb{D})$. 
Theorem 5.3.2. Let $f \in \mathbb{R}\left[\boldsymbol{\omega}_{1}, \boldsymbol{\omega}_{2}, \cdots, \boldsymbol{\omega}_{k}\right]^{S O(3, \mathbb{R})}$, then $\hat{f}$ is dual $k$ vector invariants of $S O(3, \mathbb{D})$.

Proof. We must show that $\hat{f}\left(\hat{A} \hat{\mathbf{u}}_{1}, \cdots, \hat{A} \hat{\mathbf{u}}_{k}\right)=\hat{f}\left(\hat{\mathbf{u}}_{1}, \cdots, \hat{\mathbf{u}}_{k}\right)$ where $\hat{A} \in$ $S O(3, \mathbb{D})$, and $\hat{\mathbf{u}}_{i} \in \mathbb{D}^{3}$ for all $i=1,2, \cdots, k$. Let $\hat{A}$ be defined as in Defintion 2.4.3. Suppose $\hat{\mathbf{u}}_{i}=\boldsymbol{\omega}_{i}+\epsilon \boldsymbol{v}_{i}$ for all $i=1,2, \cdots, k$, where $\boldsymbol{\omega}_{i}, \boldsymbol{v}_{i} \in \mathbb{R}^{3}$. In addition $\hat{A} \hat{\mathbf{u}}_{1}=A_{0} \boldsymbol{\omega}_{1}+\epsilon\left(A_{1} \boldsymbol{\omega}_{1}+A_{0} \boldsymbol{v}_{1}\right)$ and $\hat{A} \hat{\mathbf{u}}_{k}=A_{0} \boldsymbol{\omega}_{k}+\epsilon\left(A_{1} \boldsymbol{\omega}_{k}+A_{0} \boldsymbol{v}_{k}\right)$ $\hat{f}\left(\hat{A} \hat{\mathbf{u}}_{1}, \cdots, \hat{A} \hat{\mathbf{u}}_{k}\right)=f\left(A_{0} \boldsymbol{\omega}_{1}, \cdots, A_{0} \boldsymbol{\omega}_{k} ; A_{1} \boldsymbol{\omega}_{1}+A_{0} \boldsymbol{v}_{1}, \cdots, A_{1} \boldsymbol{\omega}_{k}+A_{0} \boldsymbol{v}_{k}\right)$ by using the dual mapping in Definition 5.2 .1 we get

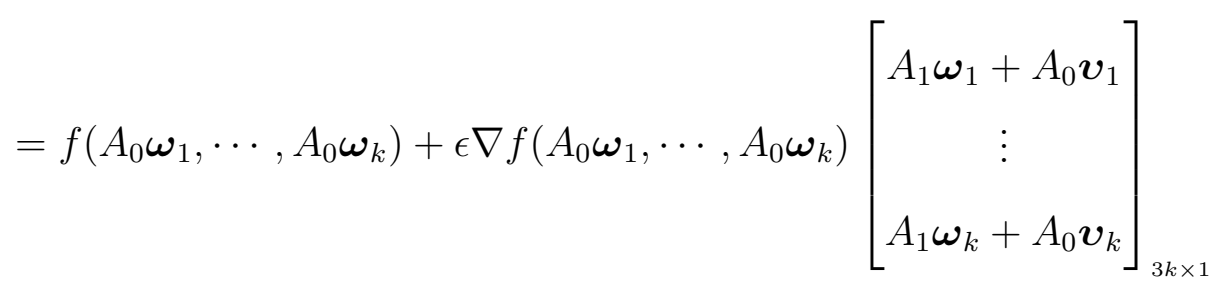

$f\left(A_{0} \boldsymbol{\omega}_{1}, \cdots, A_{0} \boldsymbol{\omega}_{k}\right)=f\left(\boldsymbol{\omega}_{1}, \cdots, \boldsymbol{\omega}_{k}\right)$, since $f$ is an $S O(3, \mathbb{R})$ invariant. Using the chain rule $\nabla f\left(A_{0} \boldsymbol{\omega}_{1}, \cdots, A_{0} \boldsymbol{\omega}_{k}\right)=\nabla f\left(\boldsymbol{\omega}_{1}, \cdots, \boldsymbol{\omega}_{k}\right) \operatorname{diag}(\underbrace{A_{0}^{t}, \cdots, A_{0}^{t}}_{k-\text { times }})$, therefore

$$
\begin{aligned}
& =f\left(\boldsymbol{\omega}_{1}, \cdots, \boldsymbol{\omega}_{k}\right)+\epsilon \nabla f\left(\boldsymbol{\omega}_{1}, \cdots, \boldsymbol{\omega}_{k}\right) \operatorname{diag}(\underbrace{A_{0}^{t}, \cdots, A_{0}^{t}}_{k-\text { times }})\left(\left[\begin{array}{c}
A_{0} \boldsymbol{v}_{1} \\
\vdots \\
A_{0} \boldsymbol{v}_{k}
\end{array}\right]+\left[\begin{array}{c}
A_{1} \boldsymbol{\omega}_{1} \\
\vdots \\
A_{1} \boldsymbol{\omega}_{k}
\end{array}\right]\right) \\
& =f\left(\boldsymbol{\omega}_{1}, \cdots, \boldsymbol{\omega}_{k}\right)+\epsilon \nabla f\left(\boldsymbol{\omega}_{1}, \cdots, \boldsymbol{\omega}_{k}\right)\left(\left[\begin{array}{c}
\boldsymbol{v}_{1} \\
\vdots \\
\boldsymbol{v}_{k}
\end{array}\right]_{3 k \times 1}+\left[\begin{array}{c}
A_{0}^{t} A_{1} \boldsymbol{\omega}_{1} \\
\vdots \\
A_{0}^{t} A_{1} \boldsymbol{\omega}_{k}
\end{array}\right]_{3 k \times 1}\right) \text {, }
\end{aligned}
$$


from Theorem 2.4.4 remember that $A_{0}^{t} A_{1}$ is skew symmetric, and from Lemma 5.1.1 $\nabla f(\boldsymbol{\omega})=\lambda \boldsymbol{\omega}^{t}$, while Lemma 2.2.1 gives us $\boldsymbol{\omega}^{t}\left(A_{0}^{t} A_{1}\right) \boldsymbol{\omega}=0$. Thus $\nabla f\left(\boldsymbol{\omega}_{1}, \cdots, \boldsymbol{\omega}_{k}\right)\left(A_{0}^{t} A_{1} \boldsymbol{\omega}_{1}, \cdots, A_{0}^{t} A_{1} \boldsymbol{\omega}_{k}\right)^{t}=0$, and hence

$$
\begin{aligned}
& =f\left(\boldsymbol{\omega}_{1}, \cdots, \boldsymbol{\omega}_{k}\right)+\epsilon \nabla f\left(\boldsymbol{\omega}_{1}, \cdots, \boldsymbol{\omega}_{k}\right)\left(\boldsymbol{v}_{1}, \cdots, \boldsymbol{v}_{k}\right)^{t} \\
& =f\left(\boldsymbol{\omega}_{1}, \cdots, \boldsymbol{\omega}_{k} ; \boldsymbol{v}_{1}, \cdots, \boldsymbol{v}_{k}\right) \\
& =\hat{f}\left(\hat{\mathbf{u}}_{1}, \cdots, \hat{\mathbf{u}}_{k}\right) .
\end{aligned}
$$

Therefore, $\hat{f}$ is dual $k$ vector invariants of $S O(3, \mathbb{D})$.

Theorem 5.3.3. If $f$ is $k$ vector invariants of $S O(3, \mathbb{R})$, then real and dual parts of $\boldsymbol{\delta}(f)=\hat{f}$ are (real) $S E(3) k$ vector invariants.

Proof. First, let us prove this theorem for one dual vector. Assume the real part of $\boldsymbol{\delta}(f)$ is $g(\boldsymbol{\omega}, \boldsymbol{v})=f(\boldsymbol{\omega})$, and the dual part of $\boldsymbol{\delta}(f)$ is $h(\boldsymbol{\omega}, \boldsymbol{v})=$ $\nabla f(\boldsymbol{\omega}) \boldsymbol{v}$, where $g, h: \mathbb{R}^{6} \longrightarrow \mathbb{R}$ are functions of Plücker coordinates acted on by $S E(3)$. Suppose $A \in S E(3)$, then $A=\left(\begin{array}{cc}A_{0} & 0 \\ A_{1} & A_{0}\end{array}\right)$.

$$
\begin{aligned}
g(A(\boldsymbol{\omega}, \boldsymbol{v})) & =g\left(\left(\begin{array}{cc}
A_{0} & 0 \\
A_{1} & A_{0}
\end{array}\right)\left(\begin{array}{l}
\boldsymbol{\omega} \\
\boldsymbol{v}
\end{array}\right)\right) \\
& =g\left(A_{0} \boldsymbol{\omega}, A_{1} \boldsymbol{\omega}+A_{0} \boldsymbol{v}\right) \\
& =f\left(A_{0} \boldsymbol{\omega}\right) .
\end{aligned}
$$

Since $g(\boldsymbol{\omega}, \boldsymbol{v})$ is an invariant of $S O(3, \mathbb{R})$ then $f\left(A_{0} \boldsymbol{\omega}\right)=f(\boldsymbol{\omega})$, and we get

$$
g(A(\boldsymbol{\omega}, \boldsymbol{v}))=g(\boldsymbol{\omega}, \boldsymbol{v})
$$


Hence, $g(\boldsymbol{\omega}, \boldsymbol{v})$ is an invariant of $S E(3, \mathbb{R})$.

$$
\begin{aligned}
h(A(\boldsymbol{\omega}, \boldsymbol{v})) & =h\left(\left(\begin{array}{ll}
A_{0} & 0 \\
A_{1} & A_{0}
\end{array}\right)\left(\begin{array}{l}
\boldsymbol{\omega} \\
\boldsymbol{v}
\end{array}\right)\right) \\
& =h\left(A_{0} \boldsymbol{\omega}, A_{1} \boldsymbol{\omega}+A_{0} \boldsymbol{v}\right) \\
& =\nabla f\left(A_{0} \boldsymbol{\omega}\right)\left(A_{1} \boldsymbol{\omega}+A_{0} \boldsymbol{v}\right) .
\end{aligned}
$$

From Lemma 5.3.1, the last formula will be

$$
=\nabla f(\boldsymbol{\omega}) \boldsymbol{v}=h(\boldsymbol{\omega}, \boldsymbol{v})
$$

Then $h(\boldsymbol{\omega}, \boldsymbol{v})$ is an invariant of $S E(3)$.

Now assume we have $k$-dual vectors invariants of $S O(3, \mathbb{D})$, then $g, h$ : $\mathbb{R}^{6} \longrightarrow \mathbb{R}$ are functions of Plücker coordinates acted on by $S E(3)$ are defined as follows

$$
\begin{aligned}
& g\left(\boldsymbol{\omega}_{1} \cdots \boldsymbol{v}_{k}\right)=f\left(\boldsymbol{\omega}_{1}, \cdots, \boldsymbol{\omega}_{k}\right) \\
& h\left(\boldsymbol{\omega}_{1} \cdots \boldsymbol{v}_{k}\right)=\nabla f\left(\boldsymbol{\omega}_{1}, \cdots \boldsymbol{\omega}_{k}\right)\left(\boldsymbol{v}_{1}, \cdots, \boldsymbol{v}_{k}\right)^{t} .
\end{aligned}
$$

We can generalise the argument above $S O(3, \mathbb{D})$ invariants of one dual vector as in the proof of Theorem 5.3.2, to prove that $g$ and $h$ are vector invariants of $S E(3)$. Hence the real and dual parts obtained from $k$-dual vector invariants of $S O(3, \mathbb{D})$ are vector invariants of the adjoint action of $S E(3)$. 


\section{Chapter 6}

\section{Dualising $S O(3, \mathbb{R})$ Vector Invariants and Syzygies}

All vector invariants of $S O(3, \mathbb{R})$ are studied and stated by Weyl [49] for multi copies of vectors. That gives us a base to study and find the vector invariants of a certain representation of other groups acting either on single or multi copies of their Lie algebra, for instance finding the vector invariants of the adjoint action of $S E(3)$ acting on multi copies of its Lie algebra, called multiscrews.

If we dualise a vector invariant of the adjoint action of $S O(3, \mathbb{R})$, then we get a dual vector invariant which is, by Theorem 5.3.2, a vector invariant of $S O(3, \mathbb{D})$. Moreover, if we split the real and dual parts of $S O(3, \mathbb{D})$ vector invariant then we get real vector invariants of the adjoint action of $S E(3)$ and Theorem 5.3 .3 assures that. The technique of dualisation for invariants first appears in Selig [47]. The following sections give more mathematical details. 


\subsection{Dualising the vector invariant of $S O(3, \mathbb{R})$ acting on a single vector}

According to Theorem 4.3.2 the vector invariant of the adjoint action of $S O(3, \mathbb{R})$ acting on single vector is $\boldsymbol{\omega} \cdot \boldsymbol{\omega}$. We will relabel $\boldsymbol{\omega} \cdot \boldsymbol{\omega}$ by $\mathbf{u} \cdot \mathbf{u}$ where $\mathbf{u} \in \mathbb{R}^{3}$. If we replace the real vector $\mathbf{u}$ by the dual vector $\hat{\mathbf{u}}=\boldsymbol{\omega}+\epsilon \boldsymbol{v}$ then,

$$
\begin{aligned}
\hat{\mathbf{u}} \cdot \hat{\mathbf{u}} & =(\boldsymbol{\omega}+\epsilon \boldsymbol{v}) \cdot(\boldsymbol{\omega}+\epsilon \boldsymbol{v}) \\
& =\boldsymbol{\omega} \cdot \boldsymbol{\omega}+2 \epsilon \boldsymbol{\omega} \cdot \boldsymbol{v}
\end{aligned}
$$

From Lemma 5.3.1 the dual number $(6.1)$ is a vector invariant of $S O(3, \mathbb{D})$. Moreover, if we split the real and dual parts, then we get the real vector invariants $\boldsymbol{\omega} \cdot \boldsymbol{\omega}$ and $\boldsymbol{\omega} \cdot \boldsymbol{v}$. According to Theorem 4.5.1 those two vector invariants generate all vector invariants of the adjoint action of $S E(3)$ acting on a single screw, and hence every invariant of the adjoint action of $S E(3)$ acting on a single screw arising from dualising the vector invariant of the adjoint action of $S O(3, \mathbb{R})$ acting on single vector belongs to $\mathbb{R}[\boldsymbol{\omega} \cdot \boldsymbol{\omega}, \boldsymbol{\omega} \cdot \boldsymbol{v}]$.

\subsection{Dualising the vector invariants of $S O(3, \mathbb{R})$ acting on two vectors}

Corollary 4.3 .2 says the vector invariants of the adjoint action of $S O(3, \mathbb{R})$ acting on two vectors $\mathbf{u}_{1}, \mathbf{u}_{2} \in \mathbb{R}^{3}$ are: $\mathbf{u}_{1} \cdot \mathbf{u}_{1}, \mathbf{u}_{2} \cdot \mathbf{u}_{2}$, and $\mathbf{u}_{1} \cdot \mathbf{u}_{2}$. If we repeat the same argument in Section 6.1 by replacing the real vectors $\mathbf{u}_{1}$, and $\mathbf{u}_{2}$ by the dual vectors $\boldsymbol{\omega}_{1}+\epsilon \boldsymbol{v}_{1}$, and $\boldsymbol{\omega}_{2}+\epsilon \boldsymbol{v}_{2}$ respectively, then we get

$$
\hat{\mathbf{u}}_{1} \cdot \hat{\mathbf{u}}_{1}=\boldsymbol{\omega}_{1} \cdot \boldsymbol{\omega}_{1}+2 \epsilon \boldsymbol{\omega}_{1} \cdot \boldsymbol{v}_{1}
$$




$$
\begin{aligned}
& \hat{\mathbf{u}}_{2} \cdot \hat{\mathbf{u}}_{2}=\boldsymbol{\omega}_{2} \cdot \boldsymbol{\omega}_{2}+2 \epsilon \boldsymbol{\omega}_{2} \cdot \boldsymbol{v}_{2} \\
& \hat{\mathbf{u}}_{1} \cdot \hat{\mathbf{u}}_{2}=\boldsymbol{\omega}_{1} \cdot \boldsymbol{\omega}_{2}+\epsilon\left(\boldsymbol{\omega}_{1} \cdot \boldsymbol{v}_{2}+\boldsymbol{v}_{1} \cdot \boldsymbol{\omega}_{2}\right) .
\end{aligned}
$$

From Lemma 5.3.1 the dual numbers 6.2 are vector invariants of $S O(3, \mathbb{D})$. Moreover, if we split the real and dual parts, then we get the real vector invariants of $S E(3)$ as we see in the following theorem.

Theorem 6.2.1. Every invariant of the adjoint action of $S E(3)$ acting on double screws arising from dualising the vector invariants of the adjoint action of $S O(3, \mathbb{R})$ acting on two vectors belongs to

$$
\begin{array}{ll}
I_{11}=\boldsymbol{\omega}_{1} \cdot \boldsymbol{\omega}_{1} & \hat{I}_{11}=\boldsymbol{\omega}_{1} \cdot \boldsymbol{v}_{1} \\
I_{22}=\boldsymbol{\omega}_{2} \cdot \boldsymbol{\omega}_{2} & \hat{I}_{22}=\boldsymbol{\omega}_{2} \cdot \boldsymbol{v}_{2} \\
I_{12}=\boldsymbol{\omega}_{1} \cdot \boldsymbol{\omega}_{2} & \hat{I}_{12}=\boldsymbol{\omega}_{1} \cdot \boldsymbol{v}_{2}+\boldsymbol{v}_{1} \cdot \boldsymbol{\omega}_{2}
\end{array}
$$

Proof. Just split the real and dual parts of 6.2, and hence $\boldsymbol{\omega}_{1} \cdot \boldsymbol{\omega}_{1}, \boldsymbol{\omega}_{2} \cdot \boldsymbol{\omega}_{2}$, $\boldsymbol{\omega}_{1} \cdot \boldsymbol{\omega}_{2}, \boldsymbol{\omega}_{1} \cdot \boldsymbol{v}_{1}, \boldsymbol{\omega}_{2} \cdot \boldsymbol{v}_{2}$ and $\boldsymbol{\omega}_{1} \cdot \boldsymbol{v}_{2}+\boldsymbol{v}_{1} \cdot \boldsymbol{\omega}_{2}$ are the vector invariants of the adjoint action of $S E(3)$. According to Theorem 4.5 .2 those vector invariants generate all invariants of the adjoint action of $S E(3)$ acting on double screws. Theorem 5.3 .3 ensures that those 6 vector invariants obtained from dualisation are vector invariants of the adjoint action of $S E(3)$. However, beside Theorem 5.3.3 let us prove those vector invariants using the basic definition of invariant given in Definition 4.1.1.

Those vector invariants that look like $\boldsymbol{\omega}_{i} \cdot \boldsymbol{\omega}_{i}$ and $\boldsymbol{\omega}_{i} \cdot \boldsymbol{v}_{i}$ where $i=1,2$ can be proved to be vector invariants of the adjoint action of $S E(3)$ acting on double screws using the same argument as in Example 4.1.4. For the rest which is $\boldsymbol{\omega}_{1} \cdot \boldsymbol{v}_{2}+\boldsymbol{v}_{1} \cdot \boldsymbol{\omega}_{2}$ here is the proof. Suppose

$$
f\left(\boldsymbol{\omega}_{1}, \boldsymbol{v}_{1}, \boldsymbol{\omega}_{2}, \boldsymbol{v}_{2}\right)=\boldsymbol{\omega}_{1} \cdot \boldsymbol{v}_{2}+\boldsymbol{v}_{1} \cdot \boldsymbol{\omega}_{2}
$$


Let $(R, \mathbf{t})$ be an element of $S E(3)$, and $\rho(R, \mathbf{t})=A$ be the adjoint action of $S E(3)$ acting on double screws, and since $A$ is a faithful representation, then by Definition 4.2 .2 we can define $A$ acting on a double screw as vector invariants such that:

$$
\left(\begin{array}{cccc}
R & 0 & 0 & 0 \\
T R & R & 0 & 0 \\
0 & 0 & R & 0 \\
0 & 0 & T R & R
\end{array}\right)\left(\begin{array}{c}
\boldsymbol{\omega}_{1} \\
\boldsymbol{v}_{1} \\
\boldsymbol{\omega}_{2} \\
\boldsymbol{v}_{2}
\end{array}\right)=\left(\begin{array}{c}
R \boldsymbol{\omega}_{1} \\
T R \boldsymbol{\omega}_{1}+R \boldsymbol{v}_{1} \\
R \boldsymbol{\omega}_{2} \\
T R \boldsymbol{\omega}_{2}+R \boldsymbol{v}_{2}
\end{array}\right)
$$

To prove $f$ is invariant. we must apply Definition 4.1.1, then we get:

$$
\begin{aligned}
f(A \mathbf{x}) & =f\left(R \boldsymbol{\omega}_{1}, T R \boldsymbol{\omega}_{1}+R \boldsymbol{v}_{1}, R \boldsymbol{\omega}_{2}, T R \boldsymbol{\omega}_{2}+R \boldsymbol{v}_{2}\right) \\
& =R \boldsymbol{\omega}_{1} \cdot\left(T R \boldsymbol{\omega}_{2}+R \boldsymbol{v}_{2}\right)+\left(T R \boldsymbol{\omega}_{1}+R \boldsymbol{v}_{1}\right) \cdot R \boldsymbol{\omega}_{2} \\
& =R \boldsymbol{\omega}_{1} \cdot T R \boldsymbol{\omega}_{2}+R \boldsymbol{\omega}_{1} \cdot R \boldsymbol{v}_{2}+T R \boldsymbol{\omega}_{1} \cdot R \boldsymbol{\omega}_{2}+R \boldsymbol{v}_{1} \cdot R \boldsymbol{\omega}_{2}
\end{aligned}
$$

Since $R \boldsymbol{\omega}_{1} \cdot T R \boldsymbol{\omega}_{2}+T R \boldsymbol{\omega}_{1} \cdot R \boldsymbol{\omega}_{2}=0$, and $R \boldsymbol{\omega}_{i} \cdot R \boldsymbol{v}_{j}=\boldsymbol{\omega}_{i} \cdot \boldsymbol{v}_{j}$, then we get:

$$
\begin{aligned}
& =\boldsymbol{\omega}_{1} \cdot \boldsymbol{v}_{2}+\boldsymbol{v}_{1} \cdot \boldsymbol{\omega}_{2} \\
& =f(\mathbf{x}) .
\end{aligned}
$$

Hence, at the top of the previous vector invariants, $\boldsymbol{\omega}_{1} \cdot \boldsymbol{v}_{2}+\boldsymbol{v}_{1} \cdot \boldsymbol{\omega}_{2}$ is a vector invariant of $S E(3)$ acting on double screws.

Dualising vector invariants of the adjoint action of $S O(3, \mathbb{R})$ acting on single or double vectors preserves the homomorphic properties like finiteness set of invariant generators. The set of invariant generators of $S O(3, \mathbb{R})$ acting on $k$-vectors is finite [49 and dualising it is still trivially finite. The vector 
invariants obtained by dualisation generate all invariants of the adjoint action of $S E(3)$ acting on either single or double screws. However, we do not have a proved theorem to assure that the vector invariants obtained by dualisation generate all invariants of the adjoint action of $S E(3)$ when acting on $k$-screws where $k \geq 3$.

\subsection{Dualising the vector invariants of $S O(3, \mathbb{R})$ acting on three vectors}

The vector invariants of the adjoint action of $S O(3, \mathbb{R})$ acting on three vectors are seven vector invariants. If we repeat the same argument Section 6.1 by replacing the real vectors $\mathbf{u}_{1}, \mathbf{u}_{2}$, and $\mathbf{u}_{3}$ by the dual vectors $\boldsymbol{\omega}_{1}+\epsilon \boldsymbol{v}_{1}, \boldsymbol{\omega}_{2}+\epsilon \boldsymbol{v}_{2}$, and $\boldsymbol{\omega}_{3}+\epsilon \boldsymbol{v}_{3}$ respectively, then we get

$$
\begin{aligned}
\hat{\mathbf{u}}_{1} \cdot \hat{\mathbf{u}}_{1} & =\boldsymbol{\omega}_{1} \cdot \boldsymbol{\omega}_{1}+2 \epsilon \boldsymbol{\omega}_{1} \cdot \boldsymbol{v}_{1} \\
\hat{\mathbf{u}}_{2} \cdot \hat{\mathbf{u}}_{2} & =\boldsymbol{\omega}_{2} \cdot \boldsymbol{\omega}_{2}+2 \epsilon \boldsymbol{\omega}_{2} \cdot \boldsymbol{v}_{2} \\
\hat{\mathbf{u}}_{3} \cdot \hat{\mathbf{u}}_{3} & =\boldsymbol{\omega}_{3} \cdot \boldsymbol{\omega}_{3}+2 \epsilon \boldsymbol{\omega}_{3} \cdot \boldsymbol{v}_{3} \\
\hat{\mathbf{u}}_{1} \cdot \hat{\mathbf{u}}_{2} & =\boldsymbol{\omega}_{1} \cdot \boldsymbol{\omega}_{2}+\epsilon\left(\boldsymbol{\omega}_{1} \cdot \boldsymbol{v}_{2}+\boldsymbol{v}_{1} \cdot \boldsymbol{\omega}_{2}\right) \\
\hat{\mathbf{u}}_{1} \cdot \hat{\mathbf{u}}_{3} & =\boldsymbol{\omega}_{1} \cdot \boldsymbol{\omega}_{3}+\epsilon\left(\boldsymbol{\omega}_{1} \cdot \boldsymbol{v}_{3}+\boldsymbol{v}_{1} \cdot \boldsymbol{\omega}_{3}\right) \\
\hat{\mathbf{u}}_{2} \cdot \hat{\mathbf{u}}_{3} & =\boldsymbol{\omega}_{2} \cdot \boldsymbol{\omega}_{3}+\epsilon\left(\boldsymbol{\omega}_{2} \cdot \boldsymbol{v}_{3}+\boldsymbol{v}_{2} \cdot \boldsymbol{\omega}_{3}\right) \\
\left|\hat{\mathbf{u}}_{1} \quad \hat{\mathbf{u}}_{2} \quad \hat{\mathbf{u}}_{3}\right| & =\left|\boldsymbol{\omega}_{1}+\epsilon \boldsymbol{v}_{1} \quad \boldsymbol{\omega}_{2}+\epsilon \boldsymbol{v}_{2} \quad \boldsymbol{\omega}_{3}+\epsilon \boldsymbol{v}_{3}\right| .
\end{aligned}
$$

By expanding the determinant, and by bearing in mind that $\epsilon^{2}=0$, we get

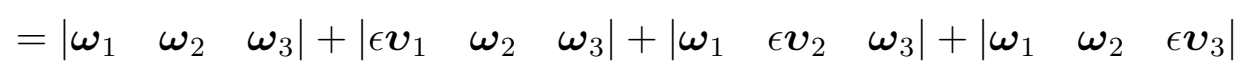

$$
\begin{aligned}
& =\left|\begin{array}{lll}
\boldsymbol{\omega}_{1} & \boldsymbol{\omega}_{2} & \boldsymbol{\omega}_{3}
\end{array}\right|+\epsilon\left(\begin{array}{llll}
\boldsymbol{v}_{1} & \boldsymbol{\omega}_{2} & \boldsymbol{\omega}_{3}
\end{array}|+| \boldsymbol{\omega}_{1} \quad \boldsymbol{v}_{2} \quad \boldsymbol{\omega}_{3}|+| \begin{array}{lll}
\boldsymbol{\omega}_{1} & \boldsymbol{\omega}_{2} & \boldsymbol{v}_{3}
\end{array} \mid\right)
\end{aligned}
$$




$$
=\left|\begin{array}{lll}
\boldsymbol{\omega}_{1} & \boldsymbol{\omega}_{2} & \boldsymbol{\omega}_{3}
\end{array}\right|+\epsilon \sum_{\sigma \in A_{3}}\left|\boldsymbol{v}_{\sigma(1)} \quad \boldsymbol{\omega}_{\sigma(2)} \quad \boldsymbol{\omega}_{\sigma(3)}\right|
$$

From Lemma 5.3 .2 the vectors in 6.3 are vector invariants of $S O(3, \mathbb{D})$. By applying Theorem 5.3 .3 we have the following theorem:

Theorem 6.3.1. The vector invariants of the adjoint action of SE(3) acting on triple screws obtained by dualising the vector invariants of the adjoint action of $S O(3, \mathbb{R})$ acting on three vectors are:

$$
\begin{aligned}
& I_{11}=\boldsymbol{\omega}_{1} \cdot \boldsymbol{\omega}_{1} \quad \hat{I}_{11}=\boldsymbol{\omega}_{1} \cdot \boldsymbol{v}_{1} \\
& I_{22}=\boldsymbol{\omega}_{2} \cdot \boldsymbol{\omega}_{2} \quad \hat{I}_{22}=\boldsymbol{\omega}_{2} \cdot \boldsymbol{v}_{2} \\
& I_{33}=\boldsymbol{\omega}_{3} \cdot \boldsymbol{\omega}_{3} \quad \hat{I}_{33}=\boldsymbol{\omega}_{3} \cdot \boldsymbol{v}_{3} \\
& I_{12}=\boldsymbol{\omega}_{1} \cdot \boldsymbol{\omega}_{2} \quad \hat{I}_{12}=\boldsymbol{\omega}_{1} \cdot \boldsymbol{v}_{2}+\boldsymbol{v}_{1} \cdot \boldsymbol{\omega}_{2} \\
& I_{13}=\boldsymbol{\omega}_{1} \cdot \boldsymbol{\omega}_{3} \quad \hat{I}_{13}=\boldsymbol{\omega}_{1} \cdot \boldsymbol{v}_{3}+\boldsymbol{v}_{1} \cdot \boldsymbol{\omega}_{3} \\
& I_{23}=\boldsymbol{\omega}_{2} \cdot \boldsymbol{\omega}_{3} \quad \hat{I}_{23}=\boldsymbol{\omega}_{2} \cdot \boldsymbol{v}_{3}+\boldsymbol{v}_{2} \cdot \boldsymbol{\omega}_{3} \\
& I_{123}=\left|\begin{array}{lll}
\boldsymbol{\omega}_{1} & \boldsymbol{\omega}_{2} & \boldsymbol{\omega}_{3}
\end{array}\right| \quad \hat{I}_{123}=\sum_{\sigma \in A_{3}}\left|\begin{array}{lll}
\boldsymbol{v}_{\sigma(1)} & \boldsymbol{\omega}_{\sigma(2)} & \boldsymbol{\omega}_{\sigma(3)}
\end{array}\right|
\end{aligned}
$$

Proof. Besides Theorem 5.3 .3 let us prove directly those vector invariants using the basic definition of invariant given in Definition 4.1.1.

Most vector invariants have been proven in Theorem 6.2.1 and Example 4.1 .4 except these two vector invariants $I_{123}$ and $\hat{I}_{123}$. Assume

$$
\begin{aligned}
f\left(\boldsymbol{\omega}_{1}, \boldsymbol{v}_{1}, \boldsymbol{\omega}_{2}, \boldsymbol{v}_{2}, \boldsymbol{\omega}_{3}, \boldsymbol{v}_{3}\right) & =\left|\begin{array}{lll}
\boldsymbol{\omega}_{1} & \boldsymbol{\omega}_{2} & \boldsymbol{\omega}_{3}
\end{array}\right|=\boldsymbol{\omega}_{1} \cdot\left(\boldsymbol{\omega}_{2} \times \boldsymbol{\omega}_{3}\right) \\
g\left(\boldsymbol{\omega}_{1}, \boldsymbol{v}_{1}, \boldsymbol{\omega}_{2}, \boldsymbol{v}_{2}, \boldsymbol{\omega}_{3}, \boldsymbol{v}_{3}\right) & =\sum_{\sigma \in A_{3}}\left|\begin{array}{lll}
\boldsymbol{v}_{\sigma(1)} & \boldsymbol{\omega}_{\sigma(2)} & \boldsymbol{\omega}_{\sigma(3)}
\end{array}\right| \\
& =\boldsymbol{v}_{1} \cdot\left(\boldsymbol{\omega}_{2} \times \boldsymbol{\omega}_{3}\right)+\boldsymbol{v}_{2} \cdot\left(\boldsymbol{\omega}_{3} \times \boldsymbol{\omega}_{1}\right)+\boldsymbol{v}_{3} \cdot\left(\boldsymbol{\omega}_{1} \times \boldsymbol{\omega}_{2}\right)
\end{aligned}
$$

Let $(R, \mathbf{t})$ be an element of $S E(3)$, and $\rho(R, \mathbf{t})=A$ is the adjoint action 
of $S E(3)$ acting on triple screws, and since $A$ is a faithful representation, then by Definition 4.2 .2 we can define $A$ acting on a triple screws as vector invariants such that:

$$
\left(\begin{array}{cccccc}
R & 0 & 0 & 0 & 0 & 0 \\
T R & R & 0 & 0 & 0 & 0 \\
0 & 0 & R & 0 & 0 & 0 \\
0 & 0 & T R & R & 0 & 0 \\
0 & 0 & 0 & 0 & R & 0 \\
0 & 0 & 0 & 0 & T R & R
\end{array}\right)\left(\begin{array}{c}
\boldsymbol{\omega}_{1} \\
\boldsymbol{v}_{1} \\
\boldsymbol{\omega}_{2} \\
\boldsymbol{v}_{2} \\
\boldsymbol{\omega}_{3} \\
\boldsymbol{v}_{3}
\end{array}\right)=\left(\begin{array}{c}
R \boldsymbol{\omega}_{1} \\
T R \boldsymbol{\omega}_{1}+R \boldsymbol{v}_{1} \\
R \boldsymbol{\omega}_{2} \\
T R \boldsymbol{\omega}_{2}+R \boldsymbol{v}_{2} \\
R \boldsymbol{\omega}_{3} \\
T R \boldsymbol{\omega}_{3}+R \boldsymbol{v}_{3}
\end{array}\right) .
$$

To prove $f$ is vector invariant, let us apply Definition 4.1.1, we get:

$$
\begin{aligned}
f(A \mathbf{x}) & =f\left(R \boldsymbol{\omega}_{1}, T R \boldsymbol{\omega}_{1}+R \boldsymbol{v}_{1}, R \boldsymbol{\omega}_{2}, T R \boldsymbol{\omega}_{2}+R \boldsymbol{v}_{2}, R \boldsymbol{\omega}_{3}, T R \boldsymbol{\omega}_{3}+R \boldsymbol{v}_{3}\right) \\
& =R \boldsymbol{\omega}_{1} \cdot\left(R \boldsymbol{\omega}_{2} \times R \boldsymbol{\omega}_{3}\right) \\
& =\boldsymbol{\omega}_{1} \cdot\left(\boldsymbol{\omega}_{2} \times \boldsymbol{\omega}_{3}\right) \\
& =f(\mathbf{x}) .
\end{aligned}
$$

To prove $g$ is invariant under the adjoint action of $S E(3)$. By using Definition 4.1.1 we get:

$$
\begin{aligned}
g(A \mathbf{x}) & =g\left(R \boldsymbol{\omega}_{1}, T R \boldsymbol{\omega}_{1}+R \boldsymbol{v}_{1}, R \boldsymbol{\omega}_{2}, T R \boldsymbol{\omega}_{2}+R \boldsymbol{v}_{2}, R \boldsymbol{\omega}_{3}, T R \boldsymbol{\omega}_{3}+R \boldsymbol{v}_{3}\right) \\
& =\left(T R \boldsymbol{\omega}_{1}+R \boldsymbol{v}_{1}\right) \cdot\left(R \boldsymbol{\omega}_{2} \times R \boldsymbol{\omega}_{3}\right)+\left(T R \boldsymbol{\omega}_{2}+R \boldsymbol{v}_{2}\right) \cdot\left(R \boldsymbol{\omega}_{3} \times R \boldsymbol{\omega}_{1}\right) \\
& +\left(T R \boldsymbol{\omega}_{3}+R \boldsymbol{v}_{3}\right) \cdot\left(R \boldsymbol{\omega}_{1} \times R \boldsymbol{\omega}_{2}\right) \\
& =T R \boldsymbol{\omega}_{1} \cdot\left(R \boldsymbol{\omega}_{2} \times R \boldsymbol{\omega}_{3}\right)+R \boldsymbol{v}_{1} \cdot\left(R \boldsymbol{\omega}_{2} \times R \boldsymbol{\omega}_{3}\right)+T R \boldsymbol{\omega}_{2} \cdot\left(R \boldsymbol{\omega}_{3} \times R \boldsymbol{\omega}_{1}\right) \\
& +R \boldsymbol{v}_{2} \cdot\left(R \boldsymbol{\omega}_{3} \times R \boldsymbol{\omega}_{1}\right)+T R \boldsymbol{\omega}_{3} \cdot\left(R \boldsymbol{\omega}_{1} \times R \boldsymbol{\omega}_{2}\right)+R \boldsymbol{v}_{3} \cdot\left(R \boldsymbol{\omega}_{1} \times R \boldsymbol{\omega}_{2}\right) .
\end{aligned}
$$


Dot and cross product are preserved under $R$, and $T \boldsymbol{\omega}_{i}=\mathbf{t} \times \boldsymbol{\omega}_{i}$ for $\mathbf{t} \in \mathbb{R}^{3}$. By applying this formula $(\mathbf{a} \times \mathbf{b}) \cdot(\mathbf{c} \times \mathbf{d})=(\mathbf{a} \cdot \mathbf{c})(\mathbf{b} \cdot \mathbf{d})-(\mathbf{a} \cdot \mathbf{d})(\mathbf{b} \cdot \mathbf{c})$, we get

$T R \boldsymbol{\omega}_{1} \cdot\left(R \boldsymbol{\omega}_{2} \times R \boldsymbol{\omega}_{3}\right)+T R \boldsymbol{\omega}_{2} \cdot\left(R \boldsymbol{\omega}_{3} \times R \boldsymbol{\omega}_{1}\right)+T R \boldsymbol{\omega}_{3} \cdot\left(R \boldsymbol{\omega}_{1} \times R \boldsymbol{\omega}_{2}\right)=0$,

and $R \boldsymbol{v}_{i} \cdot\left(R \boldsymbol{\omega}_{j} \times R \boldsymbol{\omega}_{k}\right)=\boldsymbol{v}_{i} \cdot\left(\boldsymbol{\omega}_{j} \times \boldsymbol{\omega}_{k}\right)$. Hence

$$
g(A \mathbf{x})=\boldsymbol{v}_{1} \cdot\left(\boldsymbol{\omega}_{2} \times \boldsymbol{\omega}_{3}\right)+\boldsymbol{v}_{2} \cdot\left(\boldsymbol{\omega}_{3} \times \boldsymbol{\omega}_{1}\right)+\boldsymbol{v}_{3} \cdot\left(\boldsymbol{\omega}_{1} \times \boldsymbol{\omega}_{2}\right)=g(\mathbf{x})
$$

Theorem 6.3.2. (The Jacobian Criterion for algebraic independence)[Humphreys [24], Kayal [28]]. Let $f_{1}, \cdots, f_{k} \in \mathbb{k}\left[x_{1}, \cdots, x_{n}\right.$ ] be a set of $k$ polynomials in $n$ variables over the field $\mathbb{k}$. Then these polynomials are algebraically independent if and only if the Jacobian matrix has rank $k$.

Proof. See 24, 28.

Theorem 6.3.3. The first 12 vector invariants $I_{i j}, \hat{I}_{i j}, i, j=1,2,3$ of the adjoint action of $S E(3)$ acting on triple screws are algebraically independent.

Proof. First, let us write the vectors $\boldsymbol{\omega}_{1}, \cdots, \boldsymbol{v}_{3}$ in coordinates form as follows

$$
\begin{gathered}
\boldsymbol{\omega}_{1}=\left(\begin{array}{l}
w_{11} \\
w_{12} \\
w_{13}
\end{array}\right), \quad \boldsymbol{\omega}_{2}=\left(\begin{array}{l}
w_{21} \\
w_{22} \\
w_{23}
\end{array}\right), \quad \boldsymbol{\omega}_{3}=\left(\begin{array}{l}
w_{31} \\
w_{32} \\
w_{33}
\end{array}\right), \\
\boldsymbol{v}_{1}=\left(\begin{array}{l}
v_{11} \\
v_{12} \\
v_{13}
\end{array}\right), \quad \boldsymbol{v}_{2}=\left(\begin{array}{l}
v_{21} \\
v_{22} \\
v_{23}
\end{array}\right), \quad \boldsymbol{v}_{3}=\left(\begin{array}{l}
v_{31} \\
v_{32} \\
v_{33}
\end{array}\right) .
\end{gathered}
$$


Second, let us calculate all the partial derivatives for all 12 vector invariants $I_{11}, \cdots, \hat{I}_{23}$ with respect to all 18 variables $w_{11}, \cdots, v_{33}$ and write the Jacobian matrix $J$. That is

$$
J=\left(\begin{array}{cccccccccccc}
2 w_{11} & 0 & 0 & w_{21} & w_{31} & 0 & v_{11} & 0 & 0 & v_{21} & v_{31} & 0 \\
2 w_{12} & 0 & 0 & w_{22} & w_{32} & 0 & v_{12} & 0 & 0 & v_{22} & v_{32} & 0 \\
2 w_{13} & 0 & 0 & w_{23} & w_{33} & 0 & v_{13} & 0 & 0 & v_{23} & v_{33} & 0 \\
0 & 2 w_{21} & 0 & w_{11} & 0 & w_{31} & 0 & v_{21} & 0 & v_{11} & 0 & v_{31} \\
0 & 2 w_{22} & 0 & w_{12} & 0 & w_{32} & 0 & v_{22} & 0 & v_{12} & 0 & v_{32} \\
0 & 2 w_{23} & 0 & w_{13} & 0 & w_{33} & 0 & v_{23} & 0 & v_{13} & 0 & v_{33} \\
0 & 0 & 2 w_{31} & 0 & w_{11} & w_{21} & 0 & 0 & v_{31} & 0 & v_{11} & v_{21} \\
0 & 0 & 2 w_{32} & 0 & w_{12} & w_{22} & 0 & 0 & v_{32} & 0 & v_{12} & v_{22} \\
0 & 0 & 2 w_{33} & 0 & w_{13} & w_{23} & 0 & 0 & v_{33} & 0 & v_{13} & v_{23} \\
0 & 0 & 0 & 0 & 0 & 0 & w_{11} & 0 & 0 & w_{21} & w_{31} & 0 \\
0 & 0 & 0 & 0 & 0 & 0 & w_{12} & 0 & 0 & w_{22} & w_{32} & 0 \\
0 & 0 & 0 & 0 & 0 & 0 & w_{13} & 0 & 0 & w_{23} & w_{33} & 0 \\
0 & 0 & 0 & 0 & 0 & 0 & 0 & w_{21} & 0 & w_{11} & 0 & w_{31} \\
0 & 0 & 0 & 0 & 0 & 0 & 0 & w_{22} & 0 & w_{12} & 0 & w_{32} \\
0 & 0 & 0 & 0 & 0 & 0 & 0 & w_{23} & 0 & w_{13} & 0 & w_{33} \\
0 & 0 & 0 & 0 & 0 & 0 & 0 & 0 & w_{31} & 0 & w_{11} & w_{21} \\
0 & 0 & 0 & 0 & 0 & 0 & 0 & 0 & w_{33} & 0 & w_{13} & w_{23}
\end{array}\right)
$$

By using the Maple software see Section A.1, we calculated the rank of the Jacobian matrix $J$. The rank was 12 which is equal to the number of the vector invariants, and hence by Theorem 6.3.2 the first 12 vector invariants of the adjoint action of $S E(3)$ acting on triple screws are algebraically independent. 


\subsection{Dualising $S O(3, \mathbb{R})$ syzygy}

The vector invariants of the adjoint action of $S O(3, \mathbb{R})$ acting on $k$ vectors start having syzygies when $k \geq 3$. When $k=3$ they have only one syzygy, see (4.3). In this section we dualise the relation to obtain syzygies for the adjoint action of $S E(3)$ acting on triple screws. Thus

$$
\begin{aligned}
& \left(\begin{array}{ccc}
\mid \boldsymbol{\omega}_{1}+\epsilon \boldsymbol{v}_{1} & \boldsymbol{\omega}_{2}+\epsilon \boldsymbol{v}_{2} & \left.\boldsymbol{\omega}_{3}+\epsilon \boldsymbol{v}_{3} \mid\right)^{2}= \\
\boldsymbol{\omega}_{1} \cdot \boldsymbol{\omega}_{1}+2 \epsilon \boldsymbol{\omega}_{1} \cdot \boldsymbol{v}_{1} & \boldsymbol{\omega}_{1} \cdot \boldsymbol{\omega}_{2}+\epsilon\left(\boldsymbol{\omega}_{1} \cdot \boldsymbol{v}_{2}+\boldsymbol{v}_{1} \cdot \boldsymbol{\omega}_{2}\right) & \boldsymbol{\omega}_{1} \cdot \boldsymbol{\omega}_{3}+\epsilon\left(\boldsymbol{\omega}_{1} \cdot \boldsymbol{v}_{3}+\boldsymbol{v}_{1} \cdot \boldsymbol{\omega}_{3}\right) \\
\boldsymbol{\omega}_{1} \cdot \boldsymbol{\omega}_{2}+\epsilon\left(\boldsymbol{\omega}_{1} \cdot \boldsymbol{v}_{2}+\boldsymbol{v}_{1} \cdot \boldsymbol{\omega}_{2}\right) & \boldsymbol{\omega}_{2} \cdot \boldsymbol{\omega}_{2}+2 \epsilon \boldsymbol{\omega}_{2} \cdot \boldsymbol{v}_{2} & \boldsymbol{\omega}_{2} \cdot \boldsymbol{\omega}_{3}+\epsilon\left(\boldsymbol{\omega}_{2} \cdot \boldsymbol{v}_{3}+\boldsymbol{v}_{2} \cdot \boldsymbol{\omega}_{3}\right) \\
\boldsymbol{\omega}_{1} \cdot \boldsymbol{\omega}_{3}+\epsilon\left(\boldsymbol{\omega}_{1} \cdot \boldsymbol{v}_{3}+\boldsymbol{v}_{1} \cdot \boldsymbol{\omega}_{3}\right) & \boldsymbol{\omega}_{2} \cdot \boldsymbol{\omega}_{3}+\epsilon\left(\boldsymbol{\omega}_{2} \cdot \boldsymbol{v}_{3}+\boldsymbol{v}_{2} \cdot \boldsymbol{\omega}_{3}\right) & \boldsymbol{\omega}_{3} \cdot \boldsymbol{\omega}_{3}+2 \epsilon \boldsymbol{\omega}_{3} \cdot \boldsymbol{v}_{3}
\end{array} \mid\right.
\end{aligned}
$$

Expanding the left-hand side gives:

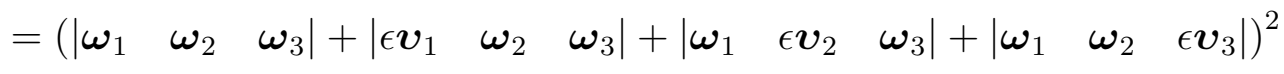

$$
\begin{aligned}
& =\left(\begin{array}{lll}
\mid \boldsymbol{\omega}_{1} & \boldsymbol{\omega}_{2} & \boldsymbol{\omega}_{3}
\end{array} \mid+\epsilon\left(\left|\begin{array}{llll}
\boldsymbol{v}_{1} & \boldsymbol{\omega}_{2} & \boldsymbol{\omega}_{3}
\end{array}\right|+\left|\boldsymbol{\omega}_{1} \quad \boldsymbol{v}_{2} \quad \boldsymbol{\omega}_{3}\right|+\left|\boldsymbol{\omega}_{1} \quad \boldsymbol{\omega}_{2} \quad \boldsymbol{v}_{3}\right|\right)\right)^{2} \\
& =\left(\begin{array}{lll}
\mid \boldsymbol{\omega}_{1} & \boldsymbol{\omega}_{2} & \boldsymbol{\omega}_{3} \mid
\end{array}\right)^{2}+2 \epsilon\left|\boldsymbol{\omega}_{1} \boldsymbol{\omega}_{2} \boldsymbol{\omega}_{3}\right|\left(\left|\boldsymbol{v}_{1} \boldsymbol{\omega}_{2} \boldsymbol{\omega}_{3}\right|+\left|\boldsymbol{\omega}_{1} \boldsymbol{v}_{2} \boldsymbol{\omega}_{3}\right|+\left|\boldsymbol{\omega}_{1} \boldsymbol{\omega}_{2} \boldsymbol{v}_{3}\right|\right)
\end{aligned}
$$

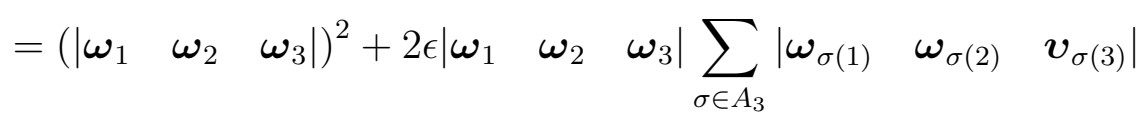

$$
\begin{aligned}
& =I_{123}^{2}+2 \epsilon I_{123} \hat{I}_{123} .
\end{aligned}
$$

while the right-hand side gives:

$$
=\left|\begin{array}{ccc}
I_{11}+2 \epsilon \hat{I}_{11} & I_{12}+\epsilon \hat{I}_{12} & I_{13}+\epsilon \hat{I}_{13} \\
I_{12}+\epsilon \hat{I}_{12} & I_{22}+2 \epsilon \hat{I}_{22} & I_{23}+\epsilon \hat{I}_{23} \\
I_{13}+\epsilon \hat{I}_{13} & I_{23}+\epsilon \hat{I}_{23} & I_{33}+2 \epsilon \hat{I}_{33}
\end{array}\right|
$$




$$
\begin{aligned}
& =\left|\begin{array}{ccc}
I_{11} & I_{12} & I_{13} \\
I_{12} & I_{22} & I_{23} \\
I_{13} & I_{23} & I_{33}
\end{array}\right|+\left|\begin{array}{ccc}
2 \epsilon \hat{I}_{11} & I_{12} & I_{13} \\
\epsilon \hat{I}_{12} & I_{22} & I_{23} \\
\epsilon \hat{I}_{13} & I_{23} & I_{33}
\end{array}\right|+\left|\begin{array}{ccc}
I_{11} & \epsilon \hat{I}_{12} & I_{13} \\
I_{12} & 2 \epsilon \hat{I}_{22} & I_{23} \\
I_{13} & \epsilon \hat{I}_{23} & I_{33}
\end{array}\right|+\left|\begin{array}{lll}
I_{11} & I_{12} & \epsilon \hat{I}_{13} \\
I_{12} & I_{22} & \epsilon \hat{I}_{23} \\
I_{13} & I_{23} & 2 \epsilon \hat{I}_{33}
\end{array}\right| \\
& =\left|\begin{array}{ccc}
I_{11} & I_{12} & I_{13} \\
I_{12} & I_{22} & I_{23} \\
I_{13} & I_{23} & I_{33}
\end{array}\right|+\epsilon\left(\left|\begin{array}{ccc}
2 \hat{I}_{11} & I_{12} & I_{13} \\
\hat{I}_{12} & I_{22} & I_{23} \\
\hat{I}_{13} & I_{23} & I_{33}
\end{array}\right|+\left|\begin{array}{ccc}
I_{11} & \hat{I}_{12} & I_{13} \\
I_{12} & 2 \hat{I}_{22} & I_{23} \\
I_{13} & \hat{I}_{23} & I_{33}
\end{array}\right|+\left|\begin{array}{ccc}
I_{11} & I_{12} & \hat{I}_{13} \\
I_{12} & I_{22} & \hat{I}_{23} \\
I_{13} & I_{23} & 2 \hat{I}_{33}
\end{array}\right|\right) \\
& =\left(I_{11} I_{22} I_{33}-I_{11} I_{23}^{2}-I_{12}^{2} I_{33}+2 I_{12} I_{13} I_{23}-I_{13}^{2} I_{22}\right)+2 \epsilon\left(\hat{I}_{11} I_{22} I_{33}-\hat{I}_{11} I_{23}^{2}\right. \\
& -\hat{I}_{22} I_{13}^{2}+\hat{I}_{22} I_{11} I_{33}+\hat{I}_{33} I_{11} I_{22}-\hat{I}_{33} I_{12}^{2}-\hat{I}_{12} I_{12} I_{33}+\hat{I}_{12} I_{13} I_{23}-\hat{I}_{13} I_{13} I_{22} \\
& \left.+\hat{I}_{13} I_{12} I_{23}+\hat{I}_{23} I_{12} I_{13}-\hat{I}_{23} I_{11} I_{23}\right) \text {. }
\end{aligned}
$$

Equating the left-hand side with the right-hand side then we get the following:

Result 6.4.1. The vector invariants $I_{123}$ and $\hat{I}_{123}$, of the adjoint action of SE(3) acting on triple screws obtained by dualising the vector invariants of the adjoint action of $S O(3, \mathbb{R})$ acting on three vectors, are not algebraically independent of $I_{i j}, \hat{I}_{i j}$ where $i, j=1,2,3$. Specifically:

(a) The first basic syzygy of $\mathrm{SE}(3)$ comes from the real part of dualising $S O(3, \mathbb{R})$ syzygy, and hence can be expressed in terms of algebraically independent vector invariants of the adjoint action of SE(3) acting on triple screws as follows

$$
\left|\omega_{1} \omega_{2} \omega_{3}\right|^{2}=I_{123}^{2}=I_{11} I_{22} I_{33}-I_{11} I_{23}^{2}-I_{12}^{2} I_{33}+2 I_{12} I_{13} I_{23}-I_{13}^{2} I_{22}
$$

(b) The second basic syzygy of $\mathrm{SE}(3)$ comes from the dual part of dualising $S O(3, \mathbb{R})$ syzygy, and hence can be expressed in terms of algebraically independent vector invariants of the adjoint action of SE(3) 
acting on triple screws as follows

$$
\begin{aligned}
I_{123} \hat{I}_{123} & =\hat{I}_{11} I_{22} I_{33}-\hat{I}_{11} I_{23}^{2}-\hat{I}_{22} I_{13}^{2}+\hat{I}_{22} I_{11} I_{33} \\
& +\hat{I}_{33} I_{11} I_{22}-\hat{I}_{33} I_{12}^{2}-\hat{I}_{12} I_{12} I_{33}+\hat{I}_{12} I_{13} I_{23} \\
& -\hat{I}_{13} I_{13} I_{22}+\hat{I}_{13} I_{12} I_{23}+\hat{I}_{23} I_{12} I_{13}-\hat{I}_{23} I_{11} I_{23} .
\end{aligned}
$$




\section{Chapter 7}

\section{Rationally Invariant Functions of the Adjoint Action of $S E(3)$ Acting on Triple Screws}

\subsection{Introduction}

In this chapter we are interested in proving that the vector invariants of the adjoint action of $S E(3)$ acting on triple screws can be expressed as rational functions of the 14 vector invariants obtained by dualising $S O(3, \mathbb{R})$ vector invariants acting on triple vectors and mentioned in Theorem 6.3.1. We follow Weyl's method of proving that functions of two arbitrary vectors $\mathbf{x}$ and $\mathbf{y}$, which are invariant under all (proper and improper) orthogonal transformations, can be expressed as polynomials of the three scalar products $\mathbf{x} \cdot \mathbf{x}$, $\mathbf{y} \cdot \mathbf{y}$ and $\mathbf{x} \cdot \mathbf{y}$. For more details, see chapter two in Weyl's book [49].

To achieve our purpose, we modify Weyl's argument to fit our group which is a semi-direct product of the orthogonal group $S O(3, \mathbb{R})$ with the real vector space $\mathbb{R}^{3}$ (see $(2.12)$ ). In particular, we use the fact that every 
even invariant and every odd invariant of the adjoint action of $E(3)$ is an absolute invariant of the adjoint action of $S E(3)$, as we prove in the next section.

\subsection{Odd and even vector invariants}

Definition 7.2.1. We define even and odd $k$-vector invariants of the adjoint action of $E(3)$ for all $R \in O(3, \mathbb{R})$ and $T \in \mathfrak{o}(3, \mathbb{R}) \cong \mathbb{R}^{3}$, as follows:

(a) $f$ is an even $k$-vector invariant polynomial in $k$ screws iff:

$$
f\left(R \boldsymbol{\omega}_{1}, T R \boldsymbol{\omega}_{1}+R \boldsymbol{v}_{1}, \cdots, R \boldsymbol{\omega}_{k}, T R \boldsymbol{\omega}_{k}+R \boldsymbol{v}_{k}\right)=f\left(\boldsymbol{\omega}_{1}, \boldsymbol{v}_{1}, \cdots, \boldsymbol{\omega}_{k}, \boldsymbol{v}_{k}\right)
$$

(b) $f$ is an odd $k$-vector invariant polynomial in $k$ screws iff:

$f\left(R \boldsymbol{\omega}_{1}, T R \boldsymbol{\omega}_{1}+R \boldsymbol{v}_{1}, \cdots, R \boldsymbol{\omega}_{k}, T R \boldsymbol{\omega}_{k}+R \boldsymbol{v}_{k}\right)=\operatorname{det}(R) f\left(\boldsymbol{\omega}_{1}, \boldsymbol{v}_{1}, \cdots, \boldsymbol{\omega}_{k}, \boldsymbol{v}_{k}\right)$

Definition 7.2.2. We define the following:

(a) $A_{E}^{(k)}$ is the set of all even $k$-vector invariants of the adjoint action of $E(3)$.

(b) $A_{O}^{(k)}$ is the set of all odd $k$-vector invariants of the adjoint action of $E(3)$.

(c) $B^{(k)}$ is the set of all absolute $k$ vector invariants of the adjoint action of $S E(3)$.

Theorem 7.2.3. For all $k, A_{E}^{(k)}+A_{O}^{(k)}=B^{(k)}$.

Proof. Let us fix $k$ and write $A_{E}^{(k)}=A_{E}, A_{O}^{(k)}=A_{O}$, and $B^{(k)}=B$. We must prove $A_{E}+A_{O} \subseteq B$ and $B \subseteq A_{E}+A_{O}$. For the first inclusion, since $S O(3, \mathbb{R})$ is a subgroup of $O(3, \mathbb{R})$, then $A_{E}, A_{O} \subseteq B$. Moreover, Proposition 
4.1.2 implies that $B$ is a $\mathbb{k}$-subalgebra so it is closed under addition, and therefore $A_{E}+A_{O} \subseteq B$.

To prove the reverse inclusion, let us assume $f \in B$, so $f$ is absolute vector invariant of the adjoint action of $S E(3)$. Define

$$
f_{(R, T)}^{\prime}\left(\boldsymbol{\omega}_{1}, \boldsymbol{v}_{1}\right)=f\left(R \boldsymbol{\omega}_{1}, T R \boldsymbol{\omega}_{1}+R \boldsymbol{v}_{1}\right)
$$

where $R \in O^{-}(3, \mathbb{R})$, and $T \in \mathfrak{o}(3, \mathbb{R})$. This element is a unique in the set of vector invariant. In order to prove that $f^{\prime}$ is the unique element, let $R_{1}, R_{2} \in O^{-}(3, \mathbb{R})$ we must show that:

$$
\begin{gathered}
f_{\left(R_{1}, T_{1}\right)}^{\prime}=f_{\left(R_{2}, T_{2}\right)}^{\prime} \\
f_{\left(R_{1}, T_{1}\right)}^{\prime}=f\left(R_{1} \boldsymbol{\omega}_{1}, T_{1} R_{1} \boldsymbol{\omega}_{1}+R_{1} \boldsymbol{v}_{1}\right), \\
=f\left(R_{1} R_{2}\left(R_{2}^{-1} \boldsymbol{\omega}_{1}\right), T_{1} R_{1} R_{2}\left(R_{2}^{-1} \boldsymbol{\omega}_{1}\right)+R_{1} R_{2}\left(R_{2}^{-1} \boldsymbol{v}_{1}\right)\right) .
\end{gathered}
$$

As a result of $R_{1}, R_{2} \in O^{-}(3, \mathbb{R})$ we have $R_{1} R_{2} \in S O(3, \mathbb{R})$, and therefore $\left(R_{1} R_{2}, T_{1}\right) \in S E(3)$. Remember that $f \in B$ is $S E(3)$ invariant. Thus

$$
\begin{aligned}
f_{\left(R_{1}, T_{1}\right)}^{\prime} & =f\left(R_{2}^{-1} \boldsymbol{\omega}_{1}, R_{2}^{-1} \boldsymbol{v}_{1}\right) \\
& =f\left(R_{2}^{2}\left(R_{2}^{-1} \boldsymbol{\omega}_{1}\right), T_{2} R_{2}^{2}\left(R_{2}^{-1} \boldsymbol{\omega}_{1}\right)+R_{2}^{2}\left(R_{2}^{-1} \boldsymbol{v}_{1}\right)\right) \\
& =f\left(R_{2} \boldsymbol{\omega}_{1}, T_{2} R_{2} \boldsymbol{\omega}_{1}+R_{2} \boldsymbol{v}_{1}\right) \\
& =f_{\left(R_{2}, T_{2}\right)}^{\prime}
\end{aligned}
$$

So for each $f \in B$, we define $f^{\prime}$ to be the unique element of the set

$$
\left\{f_{(R, T)}^{\prime} \mid R \in O^{-}(3, \mathbb{R}), T \in \mathfrak{o}(3, \mathbb{R})\right\}
$$


Let us write

$$
g_{E}=\frac{1}{2}\left(f+f^{\prime}\right), \quad \text { and } \quad g_{O}=\frac{1}{2}\left(f-f^{\prime}\right)
$$

Clearly $f=g_{E}+g_{O}$. To finish the proof we need to show that $g_{E} \in A_{E}$ and $g_{O} \in A_{O}$.

Part one: Show $g_{E} \in A_{E}$ (i.e. show $g_{E}$ is an even invariant of the adjoint action of $E(3))$. Let $R \in O(3, \mathbb{R})$ and $T \in \mathfrak{o}(3, \mathbb{R})$, then we have two different cases that should be discussed separately. When $R \in S O(3, \mathbb{R})$ and $R \in O^{-}(3, \mathbb{R})$.

Case one: Let $R \in S O(3, \mathbb{R})$. By using 7.2 and then using 7.2 , we get

$$
\begin{array}{r}
g_{E}\left(R \boldsymbol{\omega}_{1}, T R \boldsymbol{\omega}_{1}+R \boldsymbol{v}_{1}\right)=\frac{1}{2}\left[f\left(R \boldsymbol{\omega}_{1}, T R \boldsymbol{\omega}_{1}+R \boldsymbol{v}_{1}\right)+f^{\prime}\left(R \boldsymbol{\omega}_{1}, T R \boldsymbol{\omega}_{1}+R \boldsymbol{v}_{1}\right)\right] \\
=\frac{1}{2}\left[f\left(\boldsymbol{\omega}_{1}, \boldsymbol{v}_{1}\right)+f\left(R^{*}\left(R \boldsymbol{\omega}_{1}\right), T^{*} R^{*}\left(R \boldsymbol{\omega}_{1}\right)+R^{*}\left(T R \boldsymbol{\omega}_{1}+R \boldsymbol{v}_{1}\right)\right)\right]
\end{array}
$$

for any $R^{*} \in O^{-}(3, \mathbb{R})$ and $T^{*} \in \mathfrak{o}(3, \mathbb{R})$. Now let $R^{*}=-I_{3}$ and $T^{*}=0_{3 \times 3}$. Since $R \in S O(3, \mathbb{R})$ then $-R \in O^{-}(3, \mathbb{R})$. Thus, the above expression

$$
\begin{aligned}
& =\frac{1}{2}\left[f\left(\boldsymbol{\omega}_{1}, \boldsymbol{v}_{1}\right)+f\left(-R \boldsymbol{\omega}_{1}, T(-R) \boldsymbol{\omega}_{1}+(-R) \boldsymbol{v}_{1}\right)\right] \\
& =\frac{1}{2}\left[f\left(\boldsymbol{\omega}_{1}, \boldsymbol{v}_{1}\right)+f^{\prime}\left(\boldsymbol{\omega}_{1}, \boldsymbol{v}_{1}\right)\right] \\
& =g_{E}\left(\boldsymbol{\omega}_{1}, \boldsymbol{v}_{1}\right) .
\end{aligned}
$$

Case two: Let $R \in O^{-}(3, \mathbb{R})$. By using 7.2 and then using 7.2 , we get

$g_{E}\left(R \boldsymbol{\omega}_{1}, T R \boldsymbol{\omega}_{1}+R \boldsymbol{v}_{1}\right)=\frac{1}{2}\left[f\left(R \boldsymbol{\omega}_{1}, T R \boldsymbol{\omega}_{1}+R \boldsymbol{v}_{1}\right)+f^{\prime}\left(R \boldsymbol{\omega}_{1}, T R \boldsymbol{\omega}_{1}+R \boldsymbol{v}_{1}\right)\right]$ 


$$
=\frac{1}{2}\left[f^{\prime}\left(\boldsymbol{\omega}_{1}, \boldsymbol{v}_{1}\right)+f\left(R^{*}\left(R \boldsymbol{\omega}_{1}\right), T^{*} R^{*}\left(R \boldsymbol{\omega}_{1}\right)+R^{*}\left(T R \boldsymbol{\omega}_{1}+R \boldsymbol{v}_{1}\right)\right)\right],
$$

for any $R^{*} \in O^{-}(3, \mathbb{R})$ and $T^{*} \in \mathfrak{o}(3, \mathbb{R})$. Now let $R^{*}=-I_{3}$ and $T^{*}=0_{3 \times 3}$. Since $R \in O^{-}(3, \mathbb{R})$ then $-R \in S O(3, \mathbb{R})$. Thus

$$
\begin{aligned}
& =\frac{1}{2}\left[f^{\prime}\left(\boldsymbol{\omega}_{1}, \boldsymbol{v}_{1}\right)+f\left(-R \boldsymbol{\omega}_{1}, T(-R) \boldsymbol{\omega}_{1}+(-R) \boldsymbol{v}_{1}\right)\right] \\
& =\frac{1}{2}\left[f^{\prime}\left(\boldsymbol{\omega}_{1}, \boldsymbol{v}_{1}\right)+f\left(\boldsymbol{\omega}_{1}, \boldsymbol{v}_{1}\right)\right] \\
& =g_{E}\left(\boldsymbol{\omega}_{1}, \boldsymbol{v}_{1}\right) .
\end{aligned}
$$

Therefore $g_{E}\left(R \boldsymbol{\omega}_{1}, T R \boldsymbol{\omega}_{1}+R \boldsymbol{v}_{1}\right)=g_{E}\left(\boldsymbol{\omega}_{1}, \boldsymbol{v}_{1}\right)$ for all $R \in O(3, \mathbb{R})$ and $T \in$ $\mathfrak{o}(3, \mathbb{R})$, and hence $g_{E}$ is an even invariant of the adjoint action of $E(3)$.

Part two: Show $g_{O} \in A_{O}$ (i.e. show $g_{O}$ is an odd invariant of the adjoint action of $E(3))$. Let $R \in O(3, \mathbb{R})$ and $T \in \mathfrak{o}(3, \mathbb{R})$, then we have two different cases that should be discussed separately. When $R \in S O(3, \mathbb{R})$ and $R \in O^{-}(3, \mathbb{R})$.

Case one: Let $R \in S O(3, \mathbb{R})$. By using $(7.2)$ and then using $(7.2)$, we get

$$
\begin{array}{r}
g_{O}\left(R \boldsymbol{\omega}_{1}, T R \boldsymbol{\omega}_{1}+R \boldsymbol{v}_{1}\right)=\frac{1}{2}\left[f\left(R \boldsymbol{\omega}_{1}, T R \boldsymbol{\omega}_{1}+R \boldsymbol{v}_{1}\right)-f^{\prime}\left(R \boldsymbol{\omega}_{1}, T R \boldsymbol{\omega}_{1}+R \boldsymbol{v}_{1}\right)\right] \\
=\frac{1}{2}\left[f\left(\boldsymbol{\omega}_{1}, \boldsymbol{v}_{1}\right)-f\left(R^{*}\left(R \boldsymbol{\omega}_{1}\right), T^{*} R^{*}\left(R \boldsymbol{\omega}_{1}\right)+R^{*}\left(T R \boldsymbol{\omega}_{1}+R \boldsymbol{v}_{1}\right)\right)\right]
\end{array}
$$

for any $R^{*} \in O^{-}(3, \mathbb{R})$ and $T^{*} \in \mathfrak{o}(3, \mathbb{R})$. Now let $R^{*}=-I_{3}$ and $T^{*}=0_{3 \times 3}$. Since $R \in S O(3, \mathbb{R})$ then $-R \in O^{-}(3, \mathbb{R})$. Thus

$$
\begin{aligned}
& =\frac{1}{2}\left[f\left(\boldsymbol{\omega}_{1}, \boldsymbol{v}_{1}\right)-f\left(-R \boldsymbol{\omega}_{1}, T(-R) \boldsymbol{\omega}_{1}+(-R) \boldsymbol{v}_{1}\right)\right] \\
& =\frac{1}{2}\left[f\left(\boldsymbol{\omega}_{1}, \boldsymbol{v}_{1}\right)-f^{\prime}\left(\boldsymbol{\omega}_{1}, \boldsymbol{v}_{1}\right)\right]
\end{aligned}
$$




$$
=g_{O}\left(\boldsymbol{\omega}_{1}, \boldsymbol{v}_{1}\right)
$$

Case two: Let $R \in O^{-}(3, \mathbb{R})$. By using $(7.2)$ and then using $(7.2)$, we get

$$
\begin{array}{r}
g_{O}\left(R \boldsymbol{\omega}_{1}, T R \boldsymbol{\omega}_{1}+R \boldsymbol{v}_{1}\right)=\frac{1}{2}\left[f\left(R \boldsymbol{\omega}_{1}, T R \boldsymbol{\omega}_{1}+R \boldsymbol{v}_{1}\right)-f^{\prime}\left(R \boldsymbol{\omega}_{1}, T R \boldsymbol{\omega}_{1}+R \boldsymbol{v}_{1}\right)\right] \\
=\frac{1}{2}\left[f^{\prime}\left(\boldsymbol{\omega}_{1}, \boldsymbol{v}_{1}\right)+f\left(R^{*}\left(R \boldsymbol{\omega}_{1}\right), T^{*} R^{*}\left(R \boldsymbol{\omega}_{1}\right)+R^{*}\left(T R \boldsymbol{\omega}_{1}+R \boldsymbol{v}_{1}\right)\right)\right],
\end{array}
$$

for any $R^{*} \in O^{-}(3, \mathbb{R})$ and $T^{*} \in \mathfrak{o}(3, \mathbb{R})$. Now let $R^{*}=-I_{3}$ and $T^{*}=0_{3 \times 3}$. Since $R \in O^{-}(3, \mathbb{R})$ then $-R \in S O(3, \mathbb{R})$. Thus

$$
\begin{aligned}
& =\frac{1}{2}\left[f^{\prime}\left(\boldsymbol{\omega}_{1}, \boldsymbol{v}_{1}\right)-f\left(-R \boldsymbol{\omega}_{1}, T(-R) \boldsymbol{\omega}_{1}+(-R) \boldsymbol{v}_{1}\right)\right] \\
& =\frac{1}{2}\left[f^{\prime}\left(\boldsymbol{\omega}_{1}, \boldsymbol{v}_{1}\right)-f\left(\boldsymbol{\omega}_{1}, \boldsymbol{v}_{1}\right)\right] \\
& =-g_{O}\left(\boldsymbol{\omega}_{1}, \boldsymbol{v}_{1}\right) .
\end{aligned}
$$

Therefore $g_{O}\left(R \boldsymbol{\omega}_{1}, T R \boldsymbol{\omega}_{1}+R \boldsymbol{v}_{1}\right)=\operatorname{det}(R) g_{O}\left(\boldsymbol{\omega}_{1}, \boldsymbol{v}_{1}\right)$ for all $R \in O(3, \mathbb{R})$ and $T \in \mathfrak{o}(3, \mathbb{R})$, and hence $g_{O}$ is an odd invariant of the adjoint action of $E(3)$. Since $g_{O} \in A_{O}$ and $g_{E} \in A_{E}$, then $f=g_{E}+g_{O} \in A_{E}+A_{O}$, and hence $B \subseteq A_{E}+A_{O}$. The proof of the theorem is finished.

\subsection{Choice of a canonical base}

In order to express the vector invariant of the adjoint action of $S E(3)$ acting on three screws as either polynomial invariant or rationally invariant function we need to change the basis of screws $S_{i}=\left(\boldsymbol{\omega}_{i}, \boldsymbol{v}_{i}\right)$ by $S_{i}^{\prime}=\left(\boldsymbol{\omega}_{i}^{\prime}, \boldsymbol{v}_{i}^{\prime}\right)$ using 
these transformations for all $i=1,2,3$

$$
\boldsymbol{\omega}_{i} \longmapsto R \boldsymbol{\omega}_{i} \quad \boldsymbol{v}_{i} \longmapsto T R \boldsymbol{\omega}_{i}+R \boldsymbol{v}_{i}
$$

We try to write the new basis with respect to the choices of $R$, and $T$. Assume $R \boldsymbol{\omega}_{1}$ lies in the direction of $x$-axis, and $R \boldsymbol{\omega}_{2}$ lies in xy-plane, then $R \boldsymbol{\omega}_{3}$ will be any arbitrary vector in the space such that:

$$
R \boldsymbol{\omega}_{1}=\left[\begin{array}{c}
\alpha_{1} \\
0 \\
0
\end{array}\right], \quad R \boldsymbol{\omega}_{2}=\left[\begin{array}{c}
\alpha_{2} \\
\alpha_{3} \\
0
\end{array}\right], \quad R \boldsymbol{\omega}_{3}=\left[\begin{array}{c}
\alpha_{4} \\
\alpha_{5} \\
\alpha_{6}
\end{array}\right]
$$

where $\alpha_{i}$ are scalars for all $i=1, \cdots, 6$. Regarding the way we have chosen $R \boldsymbol{\omega}_{i}$, we can now determine the other transformations for $v_{i}$ 's. We want to choose the coordinates of this vector $T R \boldsymbol{\omega}_{1}+R \boldsymbol{v}_{1}$ in the way that making $y, z$ coordinates in $T R \boldsymbol{\omega}_{1}$ are equal $y, z$ coordinates in $R \boldsymbol{v}_{1}$ such that

$$
\left(T R \boldsymbol{\omega}_{1}\right)_{y}=\left(R \boldsymbol{v}_{1}\right)_{y}, \quad \text { and } \quad\left(T R \boldsymbol{\omega}_{1}\right)_{z}=\left(R \boldsymbol{v}_{1}\right)_{z}
$$

Let $T$ be defined as in Lemma 2.2.1 and $R \boldsymbol{\omega}_{1}$ as in (7.4) then

$$
T R \boldsymbol{\omega}_{1}=\left(0, \quad t_{3} \alpha_{1}, \quad-t_{2} \alpha_{1}\right)^{t}=\left(v_{x}, v_{y}, v_{z}\right)^{t}=R \boldsymbol{v}_{1}
$$

Then $t_{2}=-\frac{v_{z}}{\alpha_{1}}$ and $t_{3}=\frac{v_{y}}{\alpha_{1}}$ where $\alpha_{1} \neq 0$. From (7.6) the first coordinate of this vector $T R \boldsymbol{\omega}_{1}+R \boldsymbol{v}_{1}$ could be any arbitrary, and let us assume it $\beta_{1}$. However, the only way to make (7.5) be satisfied is to make the second and the third coordinates $T R \boldsymbol{\omega}_{1}+R \boldsymbol{v}_{1}$ be zero. Hence $T R \boldsymbol{\omega}_{1}+R \boldsymbol{v}_{1}=\left(\beta_{1}, \quad 0, \quad 0\right)^{t}$. 
For the vector $T R \boldsymbol{\omega}_{2}+R \boldsymbol{v}_{2}$ we have

$$
T R \boldsymbol{\omega}_{2}=\left(-t_{3} \alpha_{3}, \quad t_{3} \alpha_{2}, \quad t_{1} \alpha_{3}-t_{2} \alpha_{2}\right)^{t}=\left(v_{x}^{\prime}, v_{y}^{\prime}, v_{z}^{\prime}\right)^{t}=R \boldsymbol{v}_{2}
$$

Then $t_{1}=\frac{v_{z}^{\prime}+t_{2} \alpha_{2}}{\alpha_{3}}$, where $\alpha_{3} \neq 0$. The first and second coordinates of $T R \boldsymbol{\omega}_{2}+$ $R \boldsymbol{v}_{2}$ could be any arbitrary and the third coordinate must be zero and hence $T R \boldsymbol{\omega}_{2}+R \boldsymbol{v}_{2}=\left(\beta_{2}, \quad \beta_{3}, \quad 0\right)^{t}$. Vector $T R \boldsymbol{\omega}_{3}+R \boldsymbol{v}_{3}$ can be any arbitrary vector such as $T R \boldsymbol{\omega}_{3}+R \boldsymbol{v}_{3}=\left(\beta_{4}, \quad \beta_{5}, \quad \beta_{6}\right)^{t}$.

Hence the new basis, $S_{i}^{\prime}=\left(\boldsymbol{\omega}_{i}^{\prime}, \boldsymbol{v}_{i}^{\prime}\right)$ for all $i=1,2,3$, are :

$$
S_{1}^{\prime}=\left[\begin{array}{c}
\alpha_{1} \\
0 \\
0 \\
\beta_{1} \\
0 \\
0
\end{array}\right], \quad S_{2}^{\prime}=\left[\begin{array}{c}
\alpha_{2} \\
\alpha_{3} \\
0 \\
\beta_{2} \\
\beta_{3} \\
0
\end{array}\right], \quad S_{3}^{\prime}=\left[\begin{array}{c}
\alpha_{4} \\
\alpha_{5} \\
\alpha_{6} \\
\beta_{4} \\
\beta_{5} \\
\beta_{6}
\end{array}\right],
$$

where $\alpha_{i}$ and $\beta_{i}$ are scalars for all $i=1,2, \cdots, 6$.

\subsection{Even invariants of $E(3)$}

Theorem 7.4.1. The even vector invariants of the adjoint action of $E(3)$ acting on triple screws can be expressed as rational functions of these vector invariants $I_{i j}, \hat{I}_{i j}$ and $\hat{I}_{123}^{2}$ where $i, j=1,2,3$.

Proof. Suppose that $S_{i}=\left(\boldsymbol{\omega}_{i}, \boldsymbol{v}_{i}\right)$ for $i=1,2,3$ are three screws represented using Plücker coordinates, and $S_{i}^{\prime}=\left(\boldsymbol{\omega}_{i}^{\prime}, \boldsymbol{v}_{i}^{\prime}\right)$ for $i=1,2,3$ are the three new 
screws obtained by changing basis as in 7.8 . By changing basis we have

$$
f\left(S_{1}, S_{2}, S_{3}\right)=f\left(S_{1}^{\prime}, S_{2}^{\prime}, S_{3}^{\prime}\right)
$$

Using $\mathfrak{s e}(3) \cong \mathfrak{s o}(3, \mathbb{D})$ and duals of six $S O(3, \mathbb{R})$ vector invariants, then we get

$$
\begin{aligned}
S_{1} \cdot S_{1} & =S_{1}^{\prime} \cdot S_{1}^{\prime} \Longrightarrow \boldsymbol{\omega}_{1} \cdot \boldsymbol{\omega}_{1}+2 \epsilon \boldsymbol{\omega}_{1} \cdot \boldsymbol{v}_{1}=\alpha_{1}^{2}+2 \epsilon \alpha_{1} \beta_{1} \\
S_{2} \cdot S_{2} & =S_{2}^{\prime} \cdot S_{2}^{\prime} \Longrightarrow \boldsymbol{\omega}_{2} \cdot \boldsymbol{\omega}_{2}+2 \epsilon \boldsymbol{\omega}_{2} \cdot \boldsymbol{v}_{2}=\left(\alpha_{2}^{2}+\alpha_{3}^{2}\right)+2 \epsilon\left(\alpha_{2} \beta_{2}+\alpha_{3} \beta_{3}\right) \\
S_{3} \cdot S_{3} & =S_{3}^{\prime} \cdot S_{3}^{\prime} \Longrightarrow \boldsymbol{\omega}_{3} \cdot \boldsymbol{\omega}_{3}+2 \epsilon \boldsymbol{\omega}_{3} \cdot \boldsymbol{v}_{3}=\left(\alpha_{4}^{2}+\alpha_{5}^{2}+\alpha_{6}^{2}\right) \\
& +2 \epsilon\left(\alpha_{4} \beta_{4}+\alpha_{5} \beta_{5}+\alpha_{6} \beta_{6}\right) \\
S_{1} \cdot S_{2} & =S_{1}^{\prime} \cdot S_{2}^{\prime} \Longrightarrow \boldsymbol{\omega}_{1} \cdot \boldsymbol{\omega}_{2}+\epsilon\left(\boldsymbol{\omega}_{1} \cdot \boldsymbol{v}_{2}+\boldsymbol{v}_{1} \cdot \boldsymbol{\omega}_{2}\right)=\alpha_{1} \alpha_{2}+\epsilon\left(\alpha_{1} \beta_{2}+\beta_{1} \alpha_{2}\right) \\
S_{1} \cdot S_{3} & =S_{1}^{\prime} \cdot S_{3}^{\prime} \Longrightarrow \boldsymbol{\omega}_{1} \cdot \boldsymbol{\omega}_{3}+\epsilon\left(\boldsymbol{\omega}_{1} \cdot \boldsymbol{v}_{3}+\boldsymbol{v}_{1} \cdot \boldsymbol{\omega}_{3}\right)=\alpha_{1} \alpha_{4}+\epsilon\left(\alpha_{1} \beta_{4}+\beta_{1} \alpha_{4}\right) \\
S_{2} \cdot S_{3} & =S_{2}^{\prime} \cdot S_{3}^{\prime} \Longrightarrow \boldsymbol{\omega}_{2} \cdot \boldsymbol{\omega}_{3}+\epsilon\left(\boldsymbol{\omega}_{2} \cdot \boldsymbol{v}_{3}+\boldsymbol{v}_{2} \cdot \boldsymbol{\omega}_{3}\right)=\left(\alpha_{2} \alpha_{4}+\alpha_{3} \alpha_{5}\right) \\
& +\epsilon\left(\alpha_{2} \beta_{4}+\alpha_{3} \beta_{5}+\alpha_{4} \beta_{2}+\alpha_{5} \beta_{3}\right)
\end{aligned}
$$

The real part equations are:

$$
\begin{array}{lll}
\alpha_{1}^{2}=\boldsymbol{\omega}_{1} \cdot \boldsymbol{\omega}_{1}, & \alpha_{2}^{2}+\alpha_{3}^{2}=\boldsymbol{\omega}_{2} \cdot \boldsymbol{\omega}_{2}, & \alpha_{4}^{2}+\alpha_{5}^{2}+\alpha_{6}^{2}=\boldsymbol{\omega}_{3} \cdot \boldsymbol{\omega}_{3}, \\
\alpha_{1} \alpha_{2}=\boldsymbol{\omega}_{1} \cdot \boldsymbol{\omega}_{2}, & \alpha_{1} \alpha_{4}=\boldsymbol{\omega}_{1} \cdot \boldsymbol{\omega}_{3}, & \alpha_{2} \alpha_{4}+\alpha_{3} \alpha_{5}=\boldsymbol{\omega}_{2} \cdot \boldsymbol{\omega}_{3} .
\end{array}
$$

The dual part equations are:

$$
\begin{array}{ll}
\alpha_{1} \beta_{1}=\boldsymbol{\omega}_{1} \cdot \boldsymbol{v}_{1}, & \alpha_{2} \beta_{2}+\alpha_{3} \beta_{3}=\boldsymbol{\omega}_{2} \cdot \boldsymbol{v}_{2} \\
\alpha_{4} \beta_{4}+\alpha_{5} \beta_{5}+\alpha_{6} \beta_{6}=\boldsymbol{\omega}_{3} \cdot \boldsymbol{v}_{3}, & \alpha_{1} \beta_{2}+\beta_{1} \alpha_{2}=\boldsymbol{\omega}_{1} \cdot \boldsymbol{v}_{2}+\boldsymbol{v}_{1} \cdot \boldsymbol{\omega}_{2} \\
\alpha_{1} \beta_{4}+\beta_{1} \alpha_{4}=\boldsymbol{\omega}_{1} \cdot \boldsymbol{v}_{3}+\boldsymbol{v}_{1} \cdot \boldsymbol{\omega}_{3}, & \alpha_{2} \beta_{4}+\alpha_{3} \beta_{5}+\alpha_{4} \beta_{2}+\alpha_{5} \beta_{3}=\boldsymbol{\omega}_{2} \cdot \boldsymbol{v}_{3}+\boldsymbol{v}_{2} \cdot \boldsymbol{\omega}_{3} .
\end{array}
$$


By using improper rotations, that is, leave an even invariant fixed, we can additionally make any of these three transformations:

1) $\alpha_{6} \longrightarrow-\alpha_{6}$ and $\beta_{6} \longrightarrow-\beta_{6}$

2) $\alpha_{3}, \alpha_{5} \longrightarrow-\alpha_{3},-\alpha_{5}$ and $\beta_{3}, \beta_{5} \longrightarrow-\beta_{3},-\beta_{5}$

3) $\alpha_{1}, \alpha_{2}, \alpha_{4} \longrightarrow-\alpha_{1},-\alpha_{2},-\alpha_{4}$ and $\beta_{1}, \beta_{2}, \beta_{4} \longrightarrow-\beta_{1},-\beta_{2},-\beta_{4}$

The polynomial $f$ is a linear combination of monomials

$$
M=\alpha_{1}^{a_{1}} \alpha_{2}^{a_{2}} \alpha_{3}^{a_{3}} \alpha_{4}^{a_{4}} \alpha_{5}^{a_{5}} \alpha_{6}^{a_{6}} \beta_{1}^{b_{1}} \beta_{2}^{b_{2}} \beta_{3}^{b_{3}} \beta_{4}^{b_{4}} \beta_{5}^{b_{5}} \beta_{6}^{b_{6}},
$$

because of the invariance just mentioned, from first change the two exponents $a_{6}$ and $b_{6}$ are of equal parity, that is, either both even, or both odd. According to this change $M$ can be written in terms of $\alpha_{6}^{2}, \beta_{6}^{2}$, and $\alpha_{6} \beta_{6}$.

In the second change, the sum of the exponents $a_{3}, a_{5}, b_{3}$, and $b_{5}$ must be even and hence $M$ can be expressed in terms of $\alpha_{3}^{2}, \alpha_{5}^{2}, \alpha_{3} \alpha_{5}, \beta_{3}^{2}, \beta_{5}^{2}, \beta_{3} \beta_{5}$, $\alpha_{5} \beta_{5}, \alpha_{3} \beta_{3}, \alpha_{3} \beta_{5}$, and $\alpha_{5} \beta_{3}$.

In the third change, the sum of the exponents $a_{1}, a_{2}, a_{4}, b_{1}, b_{2}$, and $b_{4}$ must be even and hence $M$ is a monomial of $\alpha_{1}^{2}, \alpha_{2}^{2}, \alpha_{4}^{2}, \beta_{1}^{2}, \beta_{2}^{2}, \beta_{4}^{2}, \alpha_{1} \alpha_{2}$, $\alpha_{1} \alpha_{4}, \alpha_{2} \alpha_{4}, \beta_{1} \beta_{2}, \beta_{1} \beta_{4}, \beta_{2} \beta_{4}, \alpha_{1} \beta_{1}, \alpha_{1} \beta_{2}, \alpha_{1} \beta_{4}, \alpha_{2} \beta_{1}, \alpha_{2} \beta_{2}, \alpha_{2} \beta_{4}, \alpha_{4} \beta_{1}, \alpha_{4} \beta_{2}$, and $\alpha_{4} \beta_{4}$.

Hence, $M$ can be written in terms of all the above mentioned monomials. All those quadratics can be expressed rationally in terms of invariants $I_{11}$, $I_{22}, I_{33}, I_{12}, I_{13}, I_{23}, \hat{I}_{11}, \hat{I}_{22}, \hat{I}_{33}, \hat{I}_{12}, \hat{I}_{13}, \hat{I}_{23}$, and $\hat{I}_{123}^{2}$. It is complicated to write them down but Maple Appendix A.3 gives explicit formulas. The solution of the system of real and dual equations in terms of vector invariants 
in Theorem 7.4 .1 is

$$
\begin{aligned}
& \alpha_{1}^{2}=I_{11}, \quad \alpha_{2}^{2}=\frac{I_{12}^{2}}{I_{11}}, \quad \alpha_{3}^{2}=\frac{I_{11} I_{22}-I_{12}^{2}}{I_{11}}, \\
& \alpha_{4}^{2}=\frac{I_{13}^{2}}{I_{11}}, \quad \alpha_{5}^{2}=\frac{\left(I_{11} I_{23}-I_{12} I_{13}\right)^{2}}{\left(I_{11} I_{22}-I_{12}^{2}\right) I_{11}} \\
& \alpha_{6}^{2}=\frac{2 I_{12} I_{13} I_{23}-I_{11} I_{23}^{2}+I_{11} I_{22} I_{33}-I_{12}^{2} I_{33}-I_{22} I_{13}^{2}}{I_{11} I_{22}-I_{12}^{2}}, \\
& \beta_{1}^{2}=\frac{\hat{I}_{11}}{I_{11}}, \quad \beta_{2}^{2}=\frac{\left(\hat{I}_{12} I_{11}-\hat{I}_{11} I_{12}\right)}{I_{11}^{3}}, \\
& \beta_{3}^{2}=\frac{\left(\hat{I}_{11} I_{12}^{2}-I_{12} \hat{I}_{12} I_{11}+\hat{I}_{22} I_{11}^{2}\right)^{2}}{\left(I_{11} I_{22}-I_{12}^{2}\right) I_{11}^{3}} \\
& \beta_{4}^{2}=\frac{\left(\hat{I}_{13} I_{11}-\hat{I}_{11} I_{13}\right)^{2}}{I_{11}^{3}}, \\
& \beta_{5}^{2}=\left(\hat{I}_{11} I_{12}\left(2 I_{11} I_{22} I_{13}-I_{12}^{2} I_{13}-I_{12} I_{11} I_{23}\right)+\hat{I}_{12} I_{11}^{2}\left(I_{12} I_{23}-I_{13} I_{22}\right)\right. \\
& \frac{\left.+\hat{I}_{22} I_{11}^{2}\left(I_{12} I_{13}-I_{11} I_{23}\right)+\left(\hat{I}_{23} I_{11}^{2}-\hat{I}_{13} I_{12} I_{11}\right)\left(I_{11} I_{22}-I_{12}^{2}\right)\right)^{2}}{I_{11}^{3}\left(I_{11} I_{22}-I_{12}^{2}\right)^{3}}, \\
& \beta_{6}^{2}=\frac{\left(I_{123} \hat{I}_{123}\left(I_{11} I_{22}-I_{12}^{2}\right)-I_{123}^{2}\left(I_{11} \hat{I}_{22}+I_{22} \hat{I}_{11}-I_{12} \hat{I}_{12}\right)\right)^{2}}{\left(I_{11} I_{22}-I_{12}^{2}\right)^{3} I_{123}^{2}} \\
& =\left(I_{123}^{2} \hat{I}_{123}^{2}\left(I_{11} I_{22}-I_{12}^{2}\right)^{2}-2 I_{123}^{3} \hat{I}_{123}\left(I_{11} I_{22}\right.\right. \\
& \frac{\left.\left.-I_{12}^{2}\right)\left(I_{11} \hat{I}_{22}+I_{22} \hat{I}_{11}-I_{12} \hat{I}_{12}\right)+I_{123}^{4}\left(I_{11} \hat{I}_{22}+I_{22} \hat{I}_{11}-I_{12} \hat{I}_{12}\right)^{2}\right)}{\left(I_{11} I_{22}-I_{12}^{2}\right)^{3} I_{123}^{2}} \\
& =\left(\hat{I}_{123}^{2}\left(I_{11} I_{22}-I_{12}^{2}\right)^{2}-2 I_{123} \hat{I}_{123}\left(I_{11} I_{22}\right.\right. \\
& \frac{\left.\left.-I_{12}^{2}\right)\left(I_{11} \hat{I}_{22}+I_{22} \hat{I}_{11}-I_{12} \hat{I}_{12}\right)+I_{123}^{2}\left(I_{11} \hat{I}_{22}+I_{22} \hat{I}_{11}-I_{12} \hat{I}_{12}\right)^{2}\right)}{\left(I_{11} I_{22}-I_{12}^{2}\right)^{3}} .
\end{aligned}
$$

Note that $\beta_{6}^{2}$ has a special form requiring $\hat{I}_{123}^{2}$.

\subsection{Odd invariants of $E(3)$}

In the previous section we had twelve equations with twelve unknowns $\alpha_{i}$, $\beta_{i}$ where $i=1, \cdots, 6$. As in (7.13) each one of these twelve unknowns can 
be expressed as a function of the vector invariants $I_{11}, I_{22}, I_{33}, I_{12}, I_{13}, I_{23}$, $\hat{I}_{11}, \hat{I}_{22}, \hat{I}_{33}, \hat{I}_{12}, \hat{I}_{13}, \hat{I}_{23}$, and $\hat{I}_{123}^{2}$ that involves square roots. The following additional equations allow us to choose the correct sign for square roots. The first equation is

$$
\begin{aligned}
I_{123}=\left|\begin{array}{lll}
\boldsymbol{\omega}_{1} & \boldsymbol{\omega}_{2} & \boldsymbol{\omega}_{3}
\end{array}\right| & =\left|\begin{array}{lll}
R \boldsymbol{\omega}_{1} & R \boldsymbol{\omega}_{2} & R \boldsymbol{\omega}_{3}
\end{array}\right| \\
& =\left|\begin{array}{ccc}
\alpha_{1} & \alpha_{2} & \alpha_{4} \\
0 & \alpha_{3} & \alpha_{5} \\
0 & 0 & \alpha_{6}
\end{array}\right|=\alpha_{1} \alpha_{3} \alpha_{6} .
\end{aligned}
$$

The second equation is

$$
\begin{aligned}
& \hat{I}_{123}=\left|\begin{array}{lll}
\boldsymbol{v}_{1} & \boldsymbol{\omega}_{2} & \boldsymbol{\omega}_{3}
\end{array}\right|+\left|\begin{array}{lll}
\boldsymbol{\omega}_{1} & \boldsymbol{v}_{2} & \boldsymbol{\omega}_{3}
\end{array}\right|+\left|\begin{array}{lll}
\boldsymbol{\omega}_{1} & \boldsymbol{\omega}_{2} & \boldsymbol{v}_{3}
\end{array}\right| \\
& =\left|T R \boldsymbol{\omega}_{1}+R \boldsymbol{v}_{1} \quad R \boldsymbol{\omega}_{2} \quad R \boldsymbol{\omega}_{3}\right|+\left|R \boldsymbol{\omega}_{1} \quad T R \boldsymbol{\omega}_{2}+R \boldsymbol{v}_{2} \quad R \boldsymbol{\omega}_{3}\right| \\
& +\left|\begin{array}{lll}
R \boldsymbol{\omega}_{1} & R \boldsymbol{\omega}_{2} & T R \boldsymbol{\omega}_{3}+R \boldsymbol{v}_{3}
\end{array}\right| \\
& =\left|\begin{array}{ccc}
\beta_{1} & \alpha_{2} & \alpha_{4} \\
0 & \alpha_{3} & \alpha_{5} \\
0 & 0 & \alpha_{6}
\end{array}\right|+\left|\begin{array}{ccc}
\alpha_{1} & \beta_{2} & \alpha_{4} \\
0 & \beta_{3} & \alpha_{5} \\
0 & 0 & \alpha_{6}
\end{array}\right|+\left|\begin{array}{ccc}
\alpha_{1} & \alpha_{2} & \beta_{4} \\
0 & \alpha_{3} & \beta_{5} \\
0 & 0 & \beta_{6}
\end{array}\right| \\
& =\beta_{1} \alpha_{3} \alpha_{6}+\alpha_{1} \beta_{3} \alpha_{6}+\alpha_{1} \alpha_{3} \beta_{6} .
\end{aligned}
$$

The right-hand side of Equation 7.15 can be expressed as a rational function of $I_{11}, I_{22}, I_{33}, I_{12}, I_{13}, I_{23}, \hat{I}_{11}, \hat{I}_{22}, \hat{I}_{33}, \hat{I}_{12}, \hat{I}_{13}, \hat{I}_{23}, \hat{I}_{123}^{2}$, and $I_{123}$ as follows. If the three terms of the right-hand side of (7.15) have been multiplied and divided by $\alpha_{1}^{2}, \alpha_{3}^{2}$, and $\alpha_{6}^{2}$ respectively, we obtain

$$
\begin{aligned}
& \frac{\alpha_{1}^{2} \beta_{1} \alpha_{3} \alpha_{6}}{\alpha_{1}^{2}}+\frac{\alpha_{3}^{2} \alpha_{1} \beta_{3} \alpha_{6}}{\alpha_{3}^{2}}+\frac{\alpha_{6}^{2} \alpha_{1} \alpha_{3} \beta_{6}}{\alpha_{6}^{2}} \\
& =\frac{\left(\alpha_{1} \beta_{1}\right)\left(\alpha_{1} \alpha_{3} \alpha_{6}\right)}{\alpha_{1}^{2}}+\frac{\left(\alpha_{3} \beta_{3}\right)\left(\alpha_{1} \alpha_{3} \alpha_{6}\right)}{\alpha_{3}^{2}}+\frac{\left(\alpha_{6} \beta_{6}\right)\left(\alpha_{1} \alpha_{3} \alpha_{6}\right)}{\alpha_{6}^{2}}
\end{aligned}
$$




$$
\begin{aligned}
& =\left(\frac{\alpha_{1} \beta_{1}}{\alpha_{1}^{2}}+\frac{\alpha_{3} \beta_{3}}{\alpha_{3}^{2}}+\frac{\alpha_{6} \beta_{6}}{\alpha_{6}^{2}}\right)\left(\alpha_{1} \alpha_{3} \alpha_{6}\right) \\
& =F\left(I_{11}, I_{22}, I_{33}, I_{12}, I_{13}, I_{23}, \hat{I}_{11}, \hat{I}_{22}, \hat{I}_{33}, \hat{I}_{12}, \hat{I}_{13}, \hat{I}_{23}, \hat{I}_{123}^{2}\right) I_{123}
\end{aligned}
$$

That means the second additional equation is not needed since it can be expressed as a product of $I_{123}$ with an even rational function as in Equation 7.16 .

In Theorem 7.4.1 we showed that the product of two unknowns coming from the same set $H_{i}(i=1,2,3)$ where

$$
\begin{aligned}
& H_{1}=\left\{\alpha_{6}, \beta_{6}\right\}, \\
& H_{2}=\left\{\alpha_{3}, \alpha_{5}, \beta_{3}, \beta_{5}\right\}, \\
& H_{3}=\left\{\alpha_{1}, \alpha_{2}, \alpha_{4}, \beta_{1}, \beta_{2}, \beta_{4}\right\},
\end{aligned}
$$

can be expressed as a rational function of the first twelve algebraically independent vector invariants and $\hat{I}_{123}^{2}$. To prove a similar result for odd invariants of the adjoint action of $E(3)$, we need to prove that any product of the form $h_{1} h_{2} h_{3}$, where $h_{i} \in H_{i}$ for $i=1,2,3$, can be expressed as a rational function of $I_{11}, I_{22}, I_{33}, I_{12}, I_{13}, I_{23}, \hat{I}_{11}, \hat{I}_{22}, \hat{I}_{33}, \hat{I}_{12}, \hat{I}_{13}, \hat{I}_{23}, \hat{I}_{123}^{2}$, and $I_{123}$. There are $2 \times 4 \times 6=48$ such expressions as follows:

$$
\begin{array}{lll}
\alpha_{6} \alpha_{3} \alpha_{1}=I_{123}, & \alpha_{6} \alpha_{3} \alpha_{2}=\frac{\left(\alpha_{1} \alpha_{2}\right)}{\alpha_{1}^{2}} I_{123} \\
\alpha_{6} \alpha_{3} \alpha_{4}=\frac{\left(\alpha_{1} \alpha_{4}\right)}{\alpha_{1}^{2}} I_{123}, & \alpha_{6} \alpha_{3} \beta_{1}=\frac{\left(\alpha_{1} \beta_{1}\right)}{\alpha_{1}^{2}} I_{123} \\
\alpha_{6} \alpha_{3} \beta_{2}=\frac{\left(\alpha_{1} \beta_{2}\right)}{\alpha_{1}^{2}} I_{123}, & \alpha_{6} \alpha_{3} \beta_{4}=\frac{\left(\alpha_{1} \beta_{4}\right)}{\alpha_{1}^{2}} I_{123} \\
\alpha_{6} \alpha_{5} \alpha_{1}=\frac{\left(\alpha_{5} \alpha_{3}\right)}{\alpha_{3}^{2}} I_{123}, & \alpha_{6} \alpha_{5} \alpha_{2}=\frac{\left(\alpha_{5} \alpha_{3}\right)\left(\alpha_{2} \alpha_{1}\right)}{\alpha_{1}^{2} \alpha_{3}^{2}} I_{123} \\
\alpha_{6} \alpha_{5} \alpha_{4}=\frac{\left(\alpha_{5} \alpha_{3}\right)\left(\alpha_{4} \alpha_{1}\right)}{\alpha_{1}^{2} \alpha_{3}^{2}} I_{123}, & \alpha_{6} \alpha_{5} \beta_{1}=\frac{\left(\alpha_{5} \alpha_{3}\right)\left(\alpha_{1} \beta_{1}\right)}{\alpha_{1}^{2} \alpha_{3}^{2}} I_{123} \\
\alpha_{6} \alpha_{5} \beta_{2}=\frac{\left(\alpha_{5} \alpha_{3}\right)\left(\beta_{2} \alpha_{1}\right)}{\alpha_{1}^{2} \alpha_{3}^{2}} I_{123}, & \alpha_{6} \alpha_{5} \beta_{4}=\frac{\left(\alpha_{5} \alpha_{3}\right)\left(\beta_{4} \alpha_{1}\right)}{\alpha_{1}^{2} \alpha_{3}^{2}} I_{123}
\end{array}
$$




$$
\begin{aligned}
& \alpha_{6} \beta_{3} \alpha_{1}=\frac{\left(\beta_{3} \alpha_{3}\right)}{\alpha_{3}^{2}} I_{123}, \\
& \alpha_{6} \beta_{3} \alpha_{2}=\frac{\left(\beta_{3} \alpha_{3}\right)\left(\alpha_{2} \alpha_{1}\right)}{\alpha_{1}^{2} \alpha_{3}^{2}} I_{123} \\
& \alpha_{6} \beta_{3} \alpha_{4}=\frac{\left(\beta_{3} \alpha_{3}\right)\left(\alpha_{4} \alpha_{1}\right)}{\alpha_{1}^{2} \alpha_{3}^{2}} I_{123} \\
& \alpha_{6} \beta_{3} \beta_{1}=\frac{\left(\beta_{3} \alpha_{3}\right)\left(\alpha_{1} \beta_{1}\right)}{\alpha_{1}^{2} \alpha_{3}^{2}} I_{123} \\
& \alpha_{6} \beta_{3} \beta_{2}=\frac{\left(\beta_{3} \alpha_{3}\right)\left(\beta_{2} \alpha_{1}\right)}{\alpha_{1}^{2} \alpha_{3}^{2}} I_{123}, \\
& \alpha_{6} \beta_{3} \beta_{4}=\frac{\left(\beta_{3} \alpha_{3}\right)\left(\beta_{4} \alpha_{1}\right)}{\alpha_{1}^{2} \alpha_{3}^{2}} I_{123} \\
& \alpha_{6} \beta_{5} \alpha_{1}=\frac{\left(\beta_{5} \alpha_{3}\right)}{\alpha_{3}^{2}} I_{123}, \\
& \alpha_{6} \beta_{5} \alpha_{2}=\frac{\left(\beta_{5} \alpha_{3}\right)\left(\alpha_{2} \alpha_{1}\right)}{\alpha_{1}^{2} \alpha_{3}^{2}} I_{123} \\
& \alpha_{6} \beta_{5} \alpha_{4}=\frac{\left(\beta_{5} \alpha_{3}\right)\left(\alpha_{4} \alpha_{1}\right)}{\alpha_{1}^{2} \alpha_{3}^{2}} I_{123}, \\
& \alpha_{6} \beta_{5} \beta_{1}=\frac{\left(\beta_{5} \alpha_{3}\right)\left(\alpha_{1} \beta_{1}\right)}{\alpha_{1}^{2} \alpha_{3}^{2}} I_{123} \\
& \alpha_{6} \beta_{5} \beta_{2}=\frac{\left(\beta_{5} \alpha_{3}\right)\left(\beta_{2} \alpha_{1}\right)}{\alpha_{1}^{2} \alpha_{3}^{2}} I_{123}, \\
& \alpha_{6} \beta_{5} \beta_{4}=\frac{\left(\beta_{5} \alpha_{3}\right)\left(\beta_{4} \alpha_{1}\right)}{\alpha_{1}^{2} \alpha_{3}^{2}} I_{123} \\
& \beta_{6} \alpha_{3} \alpha_{1}=\frac{\left(\alpha_{6} \beta_{6}\right)}{\alpha_{6}^{2}} I_{123}, \\
& \beta_{6} \alpha_{3} \alpha_{2}=\frac{\left(\alpha_{6} \beta_{6}\right)\left(\alpha_{1} \alpha_{2}\right)}{\alpha_{1}^{2} \alpha_{6}^{2}} I_{123} \\
& \beta_{6} \alpha_{3} \alpha_{4}=\frac{\left(\alpha_{6} \beta_{6}\right)\left(\alpha_{1} \alpha_{4}\right)}{\alpha_{1}^{2} \alpha_{6}^{2}} I_{123}, \\
& \beta_{6} \alpha_{3} \beta_{1}=\frac{\left(\alpha_{6} \beta_{6}\right)\left(\alpha_{1} \beta_{1}\right)}{\alpha_{1}^{2} \alpha_{6}^{2}} I_{123} \\
& \beta_{6} \alpha_{3} \beta_{2}=\frac{\left(\alpha_{6} \beta_{6}\right)\left(\alpha_{1} \beta_{2}\right)}{\alpha_{1}^{2} \alpha_{6}^{2}} I_{123}, \\
& \beta_{6} \alpha_{3} \beta_{4}=\frac{\left(\alpha_{6} \beta_{6}\right)\left(\alpha_{1} \beta_{4}\right)}{\alpha_{1}^{2} \alpha_{6}^{2}} I_{123} \\
& \beta_{6} \alpha_{5} \alpha_{1}=\frac{\left(\alpha_{6} \beta_{6}\right)\left(\alpha_{5} \alpha_{3}\right)}{\alpha_{3}^{2} \alpha_{6}^{2}} I_{123}, \\
& \beta_{6} \alpha_{5} \alpha_{2}=\frac{\left(\alpha_{6} \beta_{6}\right)\left(\alpha_{5} \alpha_{3}\right)\left(\alpha_{2} \alpha_{1}\right)}{\alpha_{1}^{2} \alpha_{3}^{2} \alpha_{6}^{2}} I_{123} \\
& \beta_{6} \alpha_{5} \alpha_{4}=\frac{\left(\alpha_{6} \beta_{6}\right)\left(\alpha_{5} \alpha_{3}\right)\left(\alpha_{4} \alpha_{1}\right)}{\alpha_{1}^{2} \alpha_{3}^{2} \alpha_{6}^{2}} I_{123} \\
& \beta_{6} \alpha_{5} \beta_{1}=\frac{\left(\alpha_{6} \beta_{6}\right)\left(\alpha_{5} \alpha_{3}\right)\left(\alpha_{1} \beta_{1}\right)}{\alpha_{1}^{2} \alpha_{3}^{2} \alpha_{6}^{2}} I_{123} \\
& \beta_{6} \alpha_{5} \beta_{2}=\frac{\left(\alpha_{6} \beta_{6}\right)\left(\alpha_{5} \alpha_{3}\right)\left(\beta_{2} \alpha_{1}\right)}{\alpha_{1}^{2} \alpha_{3}^{2} \alpha_{6}^{2}} I_{123}, \\
& \beta_{6} \alpha_{5} \beta_{4}=\frac{\left(\alpha_{6} \beta_{6}\right)\left(\alpha_{5} \alpha_{3}\right)\left(\beta_{4} \alpha_{1}\right)}{\alpha_{1}^{2} \alpha_{3}^{2} \alpha_{6}^{2}} I_{123} \\
& \beta_{6} \beta_{3} \alpha_{1}=\frac{\left(\alpha_{6} \beta_{6}\right)\left(\beta_{3} \alpha_{3}\right)}{\alpha_{3}^{2} \alpha_{6}^{2}} I_{123}, \\
& \beta_{6} \beta_{3} \alpha_{2}=\frac{\left(\alpha_{6} \beta_{6}\right)\left(\beta_{3} \alpha_{3}\right)\left(\alpha_{2} \alpha_{1}\right)}{\alpha_{1}^{2} \alpha_{3}^{2} \alpha_{6}^{2}} I_{123} \\
& \beta_{6} \beta_{3} \alpha_{4}=\frac{\left(\alpha_{6} \beta_{6}\right)\left(\beta_{3} \alpha_{3}\right)\left(\alpha_{4} \alpha_{1}\right)}{\alpha_{1}^{2} \alpha_{3}^{2} \alpha_{6}^{2}} I_{123}, \\
& \beta_{6} \beta_{3} \beta_{1}=\frac{\left(\alpha_{6} \beta_{6}\right)\left(\beta_{3} \alpha_{3}\right)\left(\alpha_{1} \beta_{1}\right)}{\alpha_{1}^{2} \alpha_{3}^{2} \alpha_{6}^{2}} I_{123} \\
& \beta_{6} \beta_{3} \beta_{2}=\frac{\left(\alpha_{6} \beta_{6}\right)\left(\beta_{3} \alpha_{3}\right)\left(\beta_{2} \alpha_{1}\right)}{\alpha_{1}^{2} \alpha_{3}^{2} \alpha_{6}^{2}} I_{123}, \\
& \beta_{6} \beta_{3} \beta_{4}=\frac{\left(\alpha_{6} \beta_{6}\right)\left(\beta_{3} \alpha_{3}\right)\left(\beta_{4} \alpha_{1}\right)}{\alpha_{1}^{2} \alpha_{3}^{2} \alpha_{6}^{2}} I_{123} \\
& \beta_{6} \beta_{5} \alpha_{1}=\frac{\left(\alpha_{6} \beta_{6}\right)\left(\beta_{5} \alpha_{3}\right)}{\alpha_{3}^{2} \alpha_{6}^{2}} I_{123}, \\
& \beta_{6} \beta_{5} \alpha_{2}=\frac{\left(\alpha_{6} \beta_{6}\right)\left(\beta_{5} \alpha_{3}\right)\left(\alpha_{2} \alpha_{1}\right)}{\alpha_{1}^{2} \alpha_{3}^{2} \alpha_{6}^{2}} I_{123} \\
& \beta_{6} \beta_{5} \alpha_{4}=\frac{\left(\alpha_{6} \beta_{6}\right)\left(\beta_{5} \alpha_{3}\right)\left(\alpha_{4} \alpha_{1}\right)}{\alpha_{1}^{2} \alpha_{3}^{2} \alpha_{6}^{2}} I_{123}, \\
& \beta_{6} \beta_{5} \beta_{1}=\frac{\left(\alpha_{6} \beta_{6}\right)\left(\beta_{5} \alpha_{3}\right)\left(\alpha_{1} \beta_{1}\right)}{\alpha_{1}^{2} \alpha_{3}^{2} \alpha_{6}^{2}} I_{123} \\
& \beta_{6} \beta_{5} \beta_{2}=\frac{\left(\alpha_{6} \beta_{6}\right)\left(\beta_{5} \alpha_{3}\right)\left(\beta_{2} \alpha_{1}\right)}{\alpha_{1}^{2} \alpha_{3}^{2} \alpha_{6}^{2}} I_{123}, \quad \beta_{6} \beta_{5} \beta_{4}=\frac{\left(\alpha_{6} \beta_{6}\right)\left(\beta_{5} \alpha_{3}\right)\left(\beta_{4} \alpha_{1}\right)}{\alpha_{1}^{2} \alpha_{3}^{2} \alpha_{6}^{2}} I_{123} .
\end{aligned}
$$

Theorem 7.5.1. The odd vector invariants of the adjoint action of E(3) acting on triple screws can be expressed as rational functions of these vector invariants: $I_{i j}, \hat{I}_{i j}, I_{123}$ and $\hat{I}_{123}^{2}$ where $i, j=1,2,3$.

Proof. We know from the even case that for an invariant polynomial 
$f\left(S_{1}, S_{2}, S_{3}\right)$ can be expressed as $F\left(\alpha_{1}, \cdots, \beta_{6}\right)$. We must find all possible linear combinations of monomials $M$ mentioned in $(7.12)$ that satisfy

$$
F\left(\alpha_{1}, \cdots, \beta_{6}\right)=-F\left(\alpha_{1}^{\prime}, \cdots, \beta_{6}^{\prime}\right)
$$

where $\alpha_{1}^{\prime}, \cdots, \beta_{6}^{\prime}$ are the result of applying any of the transformations in 7.11). Let us reorder the monomial $M$ by combining unknowns $\alpha_{i}$ and $\beta_{i}$ of the same group of $H_{i}$ together, that is

$$
M=\underbrace{\alpha_{6}^{a_{6}} \beta_{6}^{b_{6}}}_{H_{1}} \underbrace{\alpha_{3}^{a_{3}} \alpha_{5}^{a_{5}} \beta_{3}^{b_{3}} \beta_{5}^{b_{5}}}_{H_{2}} \underbrace{\alpha_{1}^{a_{1}} \alpha_{2}^{a_{2}} \alpha_{4}^{a_{4}} \beta_{1}^{b_{1}} \beta_{2}^{b_{2}} \beta_{4}^{b_{4}}}_{H_{3}} .
$$

For the unknowns in $H_{1}$ to satisfy (7.19), the sum of their exponents $a_{6}$ and $b_{6}$ must be odd and thus we have two possibilities, either $\alpha_{6}^{2 n+1} \beta_{6}^{2 m}$ or $\alpha_{6}^{2 n} \beta_{6}^{2 m+1}$. However, those two combinations can be written as follows

$$
\begin{aligned}
& \alpha_{6}^{2 n+1} \beta_{6}^{2 m}=\left(\alpha_{6}^{2}\right)^{n}\left(\beta_{6}^{2}\right)^{m} \alpha_{6} \\
& \alpha_{6}^{2 n} \beta_{6}^{2 m+1}=\left(\alpha_{6}^{2}\right)^{n}\left(\beta_{6}^{2}\right)^{m} \beta_{6} .
\end{aligned}
$$

For the unknowns in $\mathrm{H}_{2}$ to satisfy (7.19), the sum of their exponents must be odd and thus we have eight possibilities as follows

$$
\begin{array}{ll}
\left(\alpha_{3}^{2}\right)^{m}\left(\alpha_{5}^{2}\right)^{n}\left(\beta_{3}^{2}\right)^{k}\left(\beta_{5}^{2}\right)^{r} \alpha_{3}, & \left(\alpha_{3}^{2}\right)^{m}\left(\alpha_{5}^{2}\right)^{n}\left(\beta_{3}^{2}\right)^{k}\left(\beta_{5}^{2}\right)^{r} \alpha_{5} \\
\left(\alpha_{3}^{2}\right)^{m}\left(\alpha_{5}^{2}\right)^{n}\left(\beta_{3}^{2}\right)^{k}\left(\beta_{5}^{2}\right)^{r} \beta_{3}, & \left(\alpha_{3}^{2}\right)^{m}\left(\alpha_{5}^{2}\right)^{n}\left(\beta_{3}^{2}\right)^{k}\left(\beta_{5}^{2}\right)^{r} \beta_{5} \\
\left(\alpha_{3}^{2}\right)^{m}\left(\alpha_{5}^{2}\right)^{n}\left(\beta_{3}^{2}\right)^{k}\left(\beta_{5}^{2}\right)^{r}\left(\alpha_{5} \beta_{3}\right) \alpha_{3}, & \left(\alpha_{3}^{2}\right)^{m}\left(\alpha_{5}^{2}\right)^{n}\left(\beta_{3}^{2}\right)^{k}\left(\beta_{5}^{2}\right)^{r}\left(\alpha_{3} \alpha_{5}\right) \beta_{5} \\
\left(\alpha_{3}^{2}\right)^{m}\left(\alpha_{5}^{2}\right)^{n}\left(\beta_{3}^{2}\right)^{k}\left(\beta_{5}^{2}\right)^{r}\left(\alpha_{3} \beta_{5}\right) \beta_{3}, & \left(\alpha_{3}^{2}\right)^{m}\left(\alpha_{5}^{2}\right)^{n}\left(\beta_{3}^{2}\right)^{k}\left(\beta_{5}^{2}\right)^{r}\left(\beta_{3} \beta_{5}\right) \alpha_{5} .
\end{array}
$$

Note that each possibility in 7.22 contains even combinations discussed in 
case two of Theorem 7.4.1 times an extra unknown, that could be any element in $\mathrm{H}_{2}$.

For the unknowns in $H_{3}$ to satisfy (7.19), the sum of their exponents must be odd and thus we have 32 possibilities, where each possibility contains even combinations as in case three of Theorem 7.4.1 times an extra unknown in $\mathrm{H}_{3}$.

Combining these three cases gives us all possibilities of monomials of the relative invariant function $F$. Each monomial consists of any of the even combinations from Theorem 7.4.1 together with three extra unknowns $h_{1} h_{2} h_{3}$ where $h_{i} \in H_{i}$. Recall from $(7.18)$ that any such combination $h_{1} h_{2} h_{3}$ can be expressed as a product of $I_{123}$ by an even rational invariant in terms of $I_{i j}$, $\hat{I}_{i j}, \hat{I}_{123}^{2}$. Hence $f$ can be rationally expressed in terms of $I_{i j}, \hat{I}_{i j}, \hat{I}_{123}^{2}$ and $I_{123}$.

Theorem 7.5.2. Every vector invariant of the adjoint action of SE(3) acting on triple screws can be expressed rationally in terms of

$$
\begin{array}{lll}
I_{i i}=\boldsymbol{\omega}_{i} \cdot \boldsymbol{\omega}_{i}, & \hat{I}_{i i}=\boldsymbol{\omega}_{i} \cdot \boldsymbol{v}_{i}, & i=1,2,3 \\
I_{i j}=\boldsymbol{\omega}_{i} \cdot \boldsymbol{\omega}_{j}, & \hat{I}_{i j}=\boldsymbol{\omega}_{i} \cdot \boldsymbol{v}_{j}+\boldsymbol{v}_{i} \cdot \boldsymbol{\omega}_{j}, & 1 \leq i<j \leq 3 \\
I_{123}=\left|\boldsymbol{\omega}_{1} \boldsymbol{\omega}_{2} \boldsymbol{\omega}_{3}\right|, & \hat{I}_{123}^{2}=\left(\left|\boldsymbol{v}_{1} \boldsymbol{\omega}_{2} \boldsymbol{\omega}_{3}\right|+\left|\boldsymbol{\omega}_{1} \boldsymbol{v}_{2} \boldsymbol{\omega}_{3}\right|+\left|\boldsymbol{\omega}_{1} \boldsymbol{\omega}_{2} \boldsymbol{v}_{3}\right|\right)^{2}
\end{array}
$$

Proof. From Theorem 7.2.3, every even invariant and every odd invariant of the adjoint action of $E(3)$ acting on triple screws is an absolute invariant of the adjoint action of $S E(3)$ acting on triple screws. Hence every vector invariant of the adjoint action of $S E(3)$ acting on triple screws can be expressed rationally in terms of $I_{11}, I_{22}, I_{33}, I_{12}, I_{13}, I_{23}, \hat{I}_{11}, \hat{I}_{22}, \hat{I}_{33}, \hat{I}_{12}, \hat{I}_{13}$, $\hat{I}_{23}, I_{123}$, and $\hat{I}_{123}^{2}$. 


\section{Chapter 8}

\section{Application to Robotics}

\subsection{Introduction}

A typical robot arm is a mechanical manipulator which can be modeled as an open articulated chain with several rigid bodies (links) connected in series by one degree of freedom joints driven by actuators. In general, there are six different possible lower-pair joints: revolute (rotary), prismatic (sliding), cylindrical, spherical, screw, and planar, mainly revolute and prismatic joints are common in manipulators.

Robot arm kinematics deals with the analytical study of the geometry of its motion with respect to a fixed reference coordinate system as a function of time without regard to forces/moments that cause the motion. Thus, it deals with the analytical description of the spatial displacement of the robot as a function of time, in particular the relations between the joint-variable space and the position and the orientation of the end-effector of a robot arm. For more details see 20 .

In this chapter we are interested in finding the link lengths and offsets of a robot arm called Denavit-Hartenberg parameters and writing them by both 
Plücker coordinates and in terms of vector invariants of the adjoint action of $S E(3)$ acting on triple screws. The aims of expressing the parameters in terms of vector invariants are; firstly it gives a simpler expression (for instance, when the offset formula is expanded in terms of Plücker coordinates it contains around 500 terms); secondly it gives us a clearer picture about what is going on and hence the algebraical significance of the parameters. Finally, we will leave the door open for more benefits that may be found by either engineers or geometers in the future.

\subsection{Denavit-Hartenberg parameters}

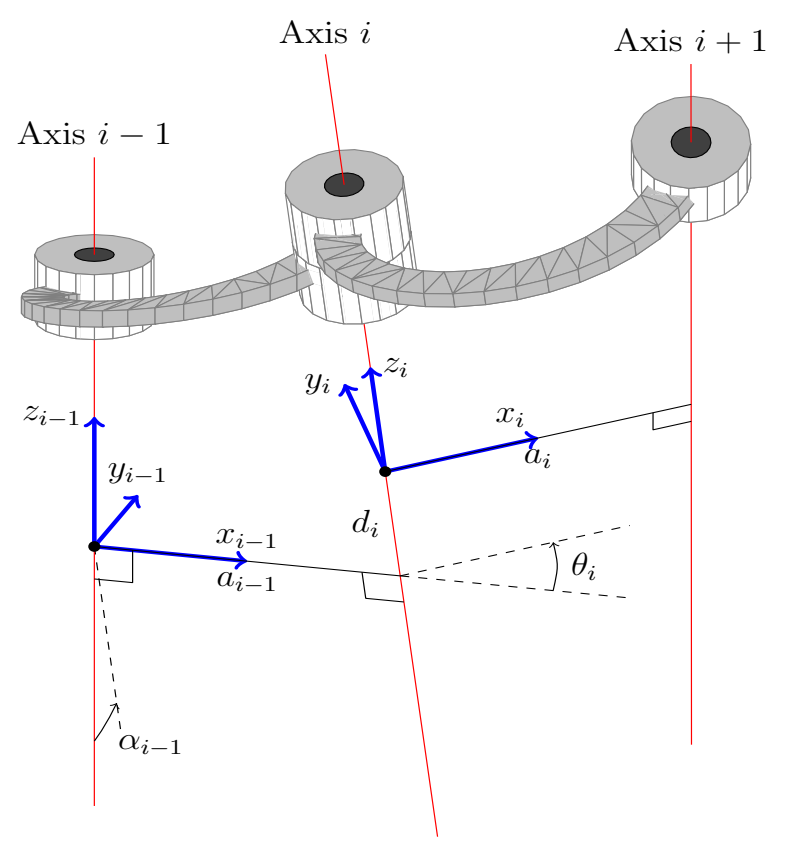

Figure 8.1: Denavit-Hartenberg frame assignment for serial robot arms 19

The Denavit-Hartenberg (DH) parameters denoted $a_{i}, d_{i}, \alpha_{i}$, and $\theta_{i}$ are the four parameters associated with a particular convention for attaching reference frames to the links of a spatial kinematic chain, or robot manipulator. 
The parameters describe how a link $(i)$ is related to link $(i-1)$, and $(i+1)$. Jacques Denavit and Richard Hartenberg introduced this convention in 1955 in order to standardize the coordinate frames for spatial linkages [23].

Richard Paul [40] demonstrated its value for the kinematic analysis of robotic systems in 1981. While many conventions for attaching references frames have been developed, the Denavit-Hartenberg convention remains the standard approach.

To understand the relation between twists and joints, relative to coordinate frames in adjacent links $i-1, i$ that coincide in the home configuration, the motion admitted by the one degree of freedom joint is given by the exponential map:

$$
\theta_{i} \mapsto \exp \left(\theta_{i} X_{i}\right) \in S E(3)
$$

For given coordinates, the defining twist $X_{i}$ of the joint is defined only up to a non-zero constant. In other words, any joint corresponds to an element of the projective Lie algebra, termed a screw.

\subsection{Link length}

The link length is the length of the common perpendicular between the axislines of two screws. Let us assume they have finite pitch and the axes of screws are non-parallel and neither joint is prismatic. In Figure 8.1 the link length between screw $i-1$ and screw $i$ is denoted by $a_{i-1}$ while the link length between screw $i$ and screw $i+1$ is denoted by $a_{i}$.

To simplify exposition, we relabel screws $i-1, i$ and $i+1$ by 1,2 and 3 and we denote those screws by $S_{1}, S_{2}$ and $S_{3}$ respectively. To calculate link length $a_{1}$ between screws $S_{1}$ and $S_{2}$ in terms of Plücker coordinates $\left(\boldsymbol{\omega}_{i}, \boldsymbol{v}_{i}\right)$ where $i=1,2$, assume the axis-line of $S_{1}$ is contained in the plane $\pi_{1}$ with 
direction vector $\boldsymbol{\omega}_{1}$ and the axis-line of $S_{2}$ is contained in the plane $\pi_{2}$ with direction vector $\boldsymbol{\omega}_{2}$. The plane $\pi_{1}$ is the plane that contains the axis for $S_{1}$ and is normal to the common perpendicular and similarly for $\pi_{2}$ with respect to $S_{2}$. Therefore $\pi_{1}$ and $\pi_{2}$ are parallel and hence they have the same normal $\boldsymbol{\omega}_{1} \times \boldsymbol{\omega}_{2}$. Assuming that the normal vector of both planes meets the first plane at point $\mathbf{x}_{1}$ and the second one at point $\mathbf{x}_{2}$. We can find these points using vector algebra that the equations of the two planes $\pi_{1}$ and $\pi_{2}$ can respectively be written in the form

$$
\begin{aligned}
& \mathbf{a} \cdot \mathbf{x}_{1}=k_{1}, \\
& \mathbf{a} \cdot \mathbf{x}_{2}=k_{2} .
\end{aligned}
$$

Here $\mathbf{a}$ is the normal vector between the two planes and is given by $\boldsymbol{\omega}_{1} \times \boldsymbol{\omega}_{2}$, while $k_{1}$ and $k_{2}$ are two scalars. The distance between the two planes is given

$$
a_{1}=\left\|\mathbf{x}_{2}-\mathbf{x}_{1}\right\|=\frac{\left|k_{2}-k_{1}\right|}{\|\mathbf{a}\|}
$$

Figure 8.2 gives a clear picture of the link length between two screws.

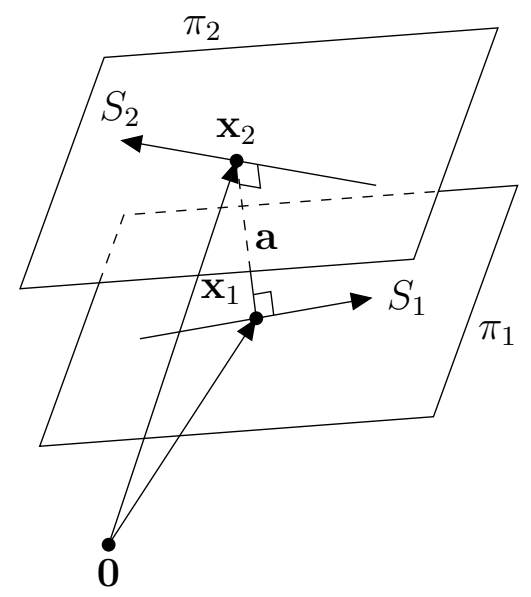

Figure 8.2: The link length between the axis-lines of two screws 
As stated before, $\mathbf{x}_{1}$ and $\mathbf{x}_{2}$ are two vectors from the origin ending at the planes $\pi_{1}$ and $\pi_{2}$ respectively (and those planes are perpendicular to the vectors $\mathbf{a}$ and $\mathbf{x}_{2}-\mathbf{x}_{1}$ and contain screws $S_{1}$ and $S_{2}$ respectively). By using (3.31), we obtain

$$
\begin{aligned}
\mathbf{x}_{1}=\frac{\boldsymbol{v}_{1} \times \boldsymbol{\omega}_{1}}{\boldsymbol{\omega}_{1} \cdot \boldsymbol{\omega}_{1}}+c_{1} \boldsymbol{\omega}_{1}, \\
\mathbf{x}_{2}=\frac{\boldsymbol{v}_{2} \times \boldsymbol{\omega}_{2}}{\boldsymbol{\omega}_{2} \cdot \boldsymbol{\omega}_{2}}+c_{2} \boldsymbol{\omega}_{2} .
\end{aligned}
$$

Substituting (8.5) and (8.6) into (8.4) and using the equality $\mathbf{a}=\boldsymbol{\omega}_{1} \times \boldsymbol{\omega}_{2}$, we get ${ }^{1}$

$$
a_{1}=\frac{\left(\boldsymbol{\omega}_{1} \times \boldsymbol{\omega}_{2}\right) \cdot \frac{\boldsymbol{v}_{1} \times \boldsymbol{\omega}_{1}}{\left\|\boldsymbol{\omega}_{1}\right\|^{2}}-\left(\boldsymbol{\omega}_{1} \times \boldsymbol{\omega}_{2}\right) \cdot \frac{\boldsymbol{v}_{2} \times \boldsymbol{\omega}_{2}}{\left\|\boldsymbol{\omega}_{2}\right\|^{2}}}{\left\|\boldsymbol{\omega}_{1} \times \boldsymbol{\omega}_{2}\right\|} .
$$

Remember for any scalars $c_{1}, c_{2}$ we have $c_{1} \mathbf{a} \cdot \boldsymbol{\omega}_{1}=c_{2} \mathbf{a} \cdot \boldsymbol{\omega}_{2}=0$. Expanding the numerator of (8.7) by using the identity of Lagrange [32]

$$
(\mathbf{a} \times \mathbf{b}) \cdot(\mathbf{c} \times \mathbf{d})=(\mathbf{a} \cdot \mathbf{c})(\mathbf{b} \cdot \mathbf{d})-(\mathbf{a} \cdot \mathbf{d})(\mathbf{b} \cdot \mathbf{c}),
$$

we get

$$
\begin{aligned}
a_{1}=\left(\left(\boldsymbol{\omega}_{1} \cdot \boldsymbol{\omega}_{2}\right)\left(\boldsymbol{v}_{1} \cdot \boldsymbol{\omega}_{1}\right)-\left(\boldsymbol{\omega}_{1} \cdot \boldsymbol{\omega}_{1}\right)\left(\boldsymbol{v}_{1} \cdot \boldsymbol{\omega}_{2}\right)\right)\left\|\boldsymbol{\omega}_{2}\right\|^{2} \\
\frac{-\left(\left(\boldsymbol{\omega}_{2} \cdot \boldsymbol{\omega}_{2}\right)\left(\boldsymbol{v}_{2} \cdot \boldsymbol{\omega}_{1}\right)-\left(\boldsymbol{\omega}_{1} \cdot \boldsymbol{\omega}_{2}\right)\left(\boldsymbol{v}_{2} \cdot \boldsymbol{\omega}_{2}\right)\right)\left\|\boldsymbol{\omega}_{1}\right\|^{2}}{\left\|\boldsymbol{\omega}_{1}\right\|^{2}\left\|\boldsymbol{\omega}_{2}\right\|^{2}\left\|\boldsymbol{\omega}_{1} \times \boldsymbol{\omega}_{2}\right\|} \\
=\frac{\boldsymbol{\omega}_{1} \cdot \boldsymbol{\omega}_{2}}{\left\|\boldsymbol{\omega}_{1} \times \boldsymbol{\omega}_{2}\right\|}\left(\frac{\boldsymbol{v}_{1} \cdot \boldsymbol{\omega}_{1}}{\left\|\boldsymbol{\omega}_{1}\right\|^{2}}+\frac{\boldsymbol{v}_{2} \cdot \boldsymbol{\omega}_{2}}{\left\|\boldsymbol{\omega}_{2}\right\|^{2}}\right)-\frac{\boldsymbol{v}_{1} \cdot \boldsymbol{\omega}_{2}+\boldsymbol{v}_{2} \cdot \boldsymbol{\omega}_{1}}{\left\|\boldsymbol{\omega}_{1} \times \boldsymbol{\omega}_{2}\right\|} \\
=\frac{\boldsymbol{\omega}_{1} \cdot \boldsymbol{\omega}_{2}}{\left\|\boldsymbol{\omega}_{1} \times \boldsymbol{\omega}_{2}\right\|}\left(\frac{\boldsymbol{v}_{1} \cdot \boldsymbol{\omega}_{1}}{\boldsymbol{\omega}_{1} \cdot \boldsymbol{\omega}_{1}}+\frac{\boldsymbol{v}_{2} \cdot \boldsymbol{\omega}_{2}}{\boldsymbol{\omega}_{2} \cdot \boldsymbol{\omega}_{2}}\right)-\frac{\boldsymbol{\omega}_{1} \cdot \boldsymbol{v}_{2}+\boldsymbol{v}_{1} \cdot \boldsymbol{\omega}_{2}}{\left\|\boldsymbol{\omega}_{1} \times \boldsymbol{\omega}_{2}\right\|}
\end{aligned}
$$

From 3.29 the ratios $\frac{\boldsymbol{v}_{1} \cdot \boldsymbol{\omega}_{1}}{\boldsymbol{\omega}_{1} \cdot \boldsymbol{\omega}_{1}}=h_{1}$ and $\frac{\boldsymbol{v}_{2} \cdot \boldsymbol{\omega}_{2}}{\boldsymbol{\omega}_{2} \cdot \boldsymbol{\omega}_{2}}=h_{2}$ represent the pitches of

\footnotetext{
${ }^{1}$ We omit the modulus sign to simplify notation. However, link length is always positive.
} 
screws $S_{1}$ and $S_{2}$ respectively. Hence

$$
a_{1}=\frac{\boldsymbol{\omega}_{1} \cdot \boldsymbol{\omega}_{2}}{\left\|\boldsymbol{\omega}_{1} \times \boldsymbol{\omega}_{2}\right\|}\left(h_{1}+h_{2}\right)-\frac{\boldsymbol{\omega}_{1} \cdot \boldsymbol{v}_{2}+\boldsymbol{v}_{1} \cdot \boldsymbol{\omega}_{2}}{\left\|\boldsymbol{\omega}_{1} \times \boldsymbol{\omega}_{2}\right\|} .
$$

We observe from (8.9) that if either $\boldsymbol{\omega}_{1}, \boldsymbol{\omega}_{2}$, or $\boldsymbol{\omega}_{1} \times \boldsymbol{\omega}_{2}$ is equal to zero, then the link length will be undefined. Using Maple we can verify that link lengths are invariants under the adjoint action of $S E(3)$, and hence link length $a_{1}$ can be expressed in terms of vector invariants of the adjoint action of $S E(3)$ acting on pairs of screws stated in Theorem 6.2.1 as below:

$$
\begin{aligned}
a_{1} & =\frac{I_{12}}{\sqrt{I_{11} I_{22}-I_{12}^{2}}}\left(\frac{\hat{I}_{11}}{I_{11}}+\frac{\hat{I}_{22}}{I_{22}}\right)-\frac{\hat{I}_{12}}{\sqrt{I_{11} I_{22}-I_{12}^{2}}} \\
& =\frac{1}{\sqrt{I_{11} I_{22}-I_{12}^{2}}}\left(\frac{\hat{I}_{11} I_{12} I_{22}+I_{11} I_{12} \hat{I}_{22}-I_{11} \hat{I}_{12} I_{22}}{I_{11} I_{22}}\right) .
\end{aligned}
$$

\subsection{Offset}

The offset is the distance between the feet of successive common perpendiculars, and it is only defined when there are three consecutive screws. In Figure 8.1 offset has been denoted by $d_{i}$. We calculate offset $d_{2}$ which lies along the axis of screw $S_{2}$. Let us assume that the three screws all have finite pitch and the axis of the middle screw should not be parallel to either of the others.

In Figure 8.3 offset $d_{2}$ precisely speaking is the distance between $\mathbf{x}_{2}$ and $\mathbf{x}_{2}^{\prime}$, which is as follows:

$$
\begin{aligned}
d_{2} & =\left\|\mathbf{x}_{2}-\mathbf{x}_{2}^{\prime}\right\| \\
& =\left\|\frac{\boldsymbol{v}_{2} \times \boldsymbol{\omega}_{2}}{\boldsymbol{\omega}_{2} \cdot \boldsymbol{\omega}_{2}}+t_{2} \boldsymbol{\omega}_{2}-\frac{\boldsymbol{v}_{2} \times \boldsymbol{\omega}_{2}}{\boldsymbol{\omega}_{2} \cdot \boldsymbol{\omega}_{2}}+t_{2}^{\prime} \boldsymbol{\omega}_{2}\right\| \\
& =\left\|t_{2} \boldsymbol{\omega}_{2}-t_{2}^{\prime} \boldsymbol{\omega}_{2}\right\|
\end{aligned}
$$




$$
=\left|t_{2}-t_{2}^{\prime}\right|\left|\boldsymbol{\omega}_{2}\right| \mid
$$

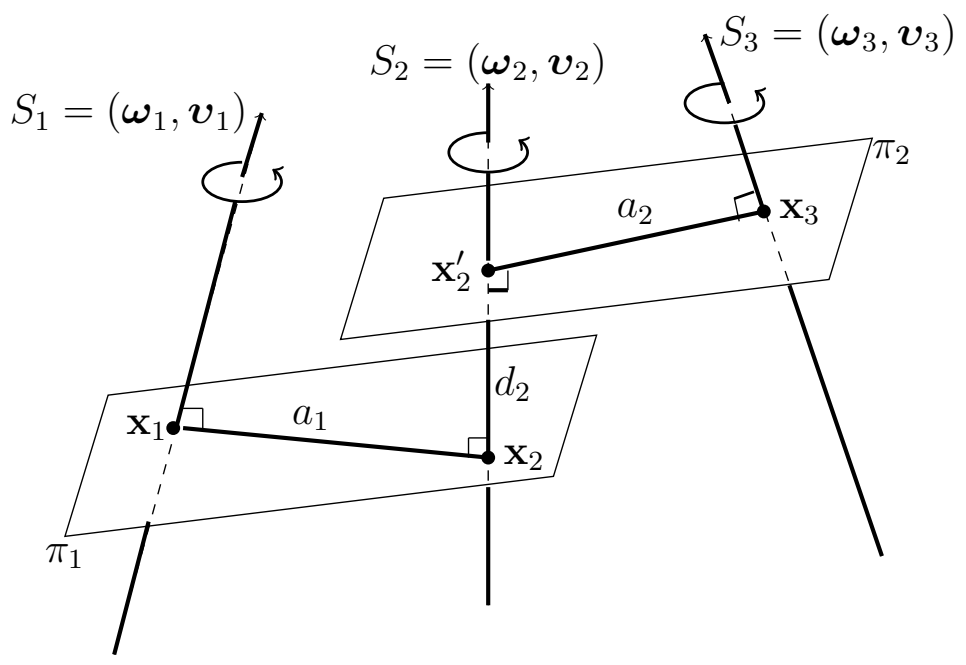

Figure 8.3: Offset between the feet of successive common perpendiculars link lengths.

We must determine $t_{2}, t_{2}^{\prime}$. Assume $\pi_{1}$, and $\pi_{2}$ are two planes containing common perpendiculars $a_{1}$, and $a_{2}$ respectively. Parametric equations of the axes $S_{1}, S_{2}$ given Plücker coordinates $\left(\boldsymbol{\omega}_{1}, \boldsymbol{v}_{1}\right)$ and $\left(\boldsymbol{\omega}_{2}, \boldsymbol{v}_{2}\right)$ respectively are:

$$
\begin{aligned}
& \mathbf{x}_{1}=\frac{\boldsymbol{v}_{1} \times \boldsymbol{\omega}_{1}}{\boldsymbol{\omega}_{1} \cdot \boldsymbol{\omega}_{1}}+t_{1} \boldsymbol{\omega}_{1}, \\
& \mathrm{x}_{2}=\frac{\boldsymbol{v}_{2} \times \boldsymbol{\omega}_{2}}{\boldsymbol{\omega}_{2} \cdot \boldsymbol{\omega}_{2}}+t_{2} \boldsymbol{\omega}_{2}, \\
& \mathbf{x}_{2}^{\prime}=\frac{\boldsymbol{v}_{2} \times \boldsymbol{\omega}_{2}}{\boldsymbol{\omega}_{2} \cdot \boldsymbol{\omega}_{2}}+t_{2}^{\prime} \boldsymbol{\omega}_{2} .
\end{aligned}
$$

The fact that $\mathbf{x}_{2}-\mathbf{x}_{1}$ is perpendicular to axes of screws $S_{1}$ and $S_{2}$ gives us these two equations:

$$
\left(\frac{\boldsymbol{v}_{2} \times \boldsymbol{\omega}_{2}}{\boldsymbol{\omega}_{2} \cdot \boldsymbol{\omega}_{2}}+t_{2} \boldsymbol{\omega}_{2}-\frac{\boldsymbol{v}_{1} \times \boldsymbol{\omega}_{1}}{\boldsymbol{\omega}_{1} \cdot \boldsymbol{\omega}_{1}}-t_{1} \boldsymbol{\omega}_{1}\right) \cdot \boldsymbol{\omega}_{1}=0
$$




$$
\left(\frac{\boldsymbol{v}_{2} \times \boldsymbol{\omega}_{2}}{\boldsymbol{\omega}_{2} \cdot \boldsymbol{\omega}_{2}}+t_{2} \boldsymbol{\omega}_{2}-\frac{\boldsymbol{v}_{1} \times \boldsymbol{\omega}_{1}}{\boldsymbol{\omega}_{1} \cdot \boldsymbol{\omega}_{1}}-t_{1} \boldsymbol{\omega}_{1}\right) \cdot \boldsymbol{\omega}_{2}=0
$$

By distributing the dot product over both equations and noting that $\boldsymbol{v}_{1} \times \boldsymbol{\omega}_{1}$ and $\boldsymbol{\omega}_{1}$ are orthogonal, similarly, $\boldsymbol{v}_{2} \times \boldsymbol{\omega}_{2}$ and $\boldsymbol{\omega}_{2}$, then :

$$
\begin{aligned}
& \left(\frac{\left(\boldsymbol{v}_{2} \times \boldsymbol{\omega}_{2}\right) \cdot \boldsymbol{\omega}_{1}}{\boldsymbol{\omega}_{2} \cdot \boldsymbol{\omega}_{2}}\right)+t_{2}\left(\boldsymbol{\omega}_{2} \cdot \boldsymbol{\omega}_{1}\right)-\left(\frac{\left(\boldsymbol{v}_{1} \times \boldsymbol{\omega}_{1}\right) \cdot \boldsymbol{\omega}_{1}}{\boldsymbol{\omega}_{1} \cdot \boldsymbol{\omega}_{1}}\right)^{0}-t_{1}\left(\boldsymbol{\omega}_{1} \cdot \boldsymbol{\omega}_{1}\right)=0 \\
& \left(\frac{\left(\boldsymbol{v}_{2} \times \boldsymbol{\omega}_{2}\right) \cdot \boldsymbol{\omega}_{2}}{\boldsymbol{\omega}_{2} \cdot \boldsymbol{\omega}_{2}}\right)^{0}+t_{2}\left(\boldsymbol{\omega}_{2} \cdot \boldsymbol{\omega}_{2}\right)-\left(\frac{\left(\boldsymbol{v}_{1} \times \boldsymbol{\omega}_{1}\right) \cdot \boldsymbol{\omega}_{2}}{\boldsymbol{\omega}_{1} \cdot \boldsymbol{\omega}_{1}}\right)-t_{1}\left(\boldsymbol{\omega}_{1} \cdot \boldsymbol{\omega}_{2}\right)=0
\end{aligned}
$$

Eliminating $t_{1}$, then $t_{2}$ can be obtained as follows:

$$
\begin{aligned}
t_{2} & \left(\left(\boldsymbol{\omega}_{1} \cdot \boldsymbol{\omega}_{1}\right)\left(\boldsymbol{\omega}_{2} \cdot \boldsymbol{\omega}_{2}\right)-\left(\boldsymbol{\omega}_{1} \cdot \boldsymbol{\omega}_{2}\right)^{2}\right) \\
\quad= & \frac{\left(\boldsymbol{v}_{2} \times \boldsymbol{\omega}_{2}\right) \cdot \boldsymbol{\omega}_{1}}{\boldsymbol{\omega}_{2} \cdot \boldsymbol{\omega}_{2}}\left(\boldsymbol{\omega}_{1} \cdot \boldsymbol{\omega}_{2}\right)+\left(\boldsymbol{v}_{1} \times \boldsymbol{\omega}_{1}\right) \cdot \boldsymbol{\omega}_{2}
\end{aligned}
$$

Using (8.8) and making $t_{2}$ subject of the rule, then we get:

$$
t_{2}=\frac{\left(\left(\boldsymbol{v}_{2} \times \boldsymbol{\omega}_{2}\right) \cdot \boldsymbol{\omega}_{1}\right)\left(\boldsymbol{\omega}_{1} \cdot \boldsymbol{\omega}_{2}\right)+\left(\left(\boldsymbol{v}_{1} \times \boldsymbol{\omega}_{1}\right) \cdot \boldsymbol{\omega}_{2}\right)\left(\boldsymbol{\omega}_{2} \cdot \boldsymbol{\omega}_{2}\right)}{\left\|\boldsymbol{\omega}_{2}\right\|^{2}\left\|\boldsymbol{\omega}_{1} \times \boldsymbol{\omega}_{2}\right\|^{2}}
$$

In order to find $t_{2}^{\prime}$ we can repeat the previous argument between the second screw and third screw instead of first and second screws. However, we can more simply find $t_{2}^{\prime}$ from $t_{2}$ by replacing index 1 by index 3 and hence obtain $t_{2}^{\prime}$ as below:

$$
t_{2}^{\prime}=\frac{\left(\left(\boldsymbol{v}_{2} \times \boldsymbol{\omega}_{2}\right) \cdot \boldsymbol{\omega}_{3}\right)\left(\boldsymbol{\omega}_{3} \cdot \boldsymbol{\omega}_{2}\right)+\left(\left(\boldsymbol{v}_{3} \times \boldsymbol{\omega}_{3}\right) \cdot \boldsymbol{\omega}_{2}\right)\left(\boldsymbol{\omega}_{2} \cdot \boldsymbol{\omega}_{2}\right)}{\left\|\boldsymbol{\omega}_{2}\right\|^{2}\left\|\boldsymbol{\omega}_{3} \times \boldsymbol{\omega}_{2}\right\|^{2}}
$$

Substituting 8.22 and 8.23 in 8.13 , and remembering that 
$\left(\left(\boldsymbol{v}_{2} \times \boldsymbol{\omega}_{2}\right) \cdot \boldsymbol{\omega}_{3}\right)$ is a determinant, so we can write it as $\left|\boldsymbol{v}_{2} \boldsymbol{\omega}_{2} \boldsymbol{\omega}_{3}\right|$, gives:

$d_{2}=\left|\frac{\left|\boldsymbol{v}_{2} \boldsymbol{\omega}_{2} \boldsymbol{\omega}_{1}\right|\left(\boldsymbol{\omega}_{1} \cdot \boldsymbol{\omega}_{2}\right)+\left|\boldsymbol{v}_{1} \boldsymbol{\omega}_{1} \boldsymbol{\omega}_{2}\right| \mid \boldsymbol{\omega}_{2} \|^{2}}{\left\|\boldsymbol{\omega}_{2}\right\|^{2}\left\|\boldsymbol{\omega}_{1} \times \boldsymbol{\omega}_{2}\right\|^{2}}-\frac{\left|\boldsymbol{v}_{2} \boldsymbol{\omega}_{2} \boldsymbol{\omega}_{3}\right|\left(\boldsymbol{\omega}_{3} \cdot \boldsymbol{\omega}_{2}\right)+\left|\boldsymbol{v}_{3} \boldsymbol{\omega}_{3} \boldsymbol{\omega}_{2}\right|\left\|\boldsymbol{\omega}_{2}\right\|^{2}}{\left\|\boldsymbol{\omega}_{2}\right\|^{2}\left\|\boldsymbol{\omega}_{3} \times \boldsymbol{\omega}_{2}\right\|^{2}}\right|\left\|\boldsymbol{\omega}_{2}\right\|$.

By making a common denominator we get: ${ }^{2}$

$$
\begin{aligned}
& d_{2}=\left(\left|\boldsymbol{v}_{2} \boldsymbol{\omega}_{2} \boldsymbol{\omega}_{1}\right|\left(\boldsymbol{\omega}_{1} \cdot \boldsymbol{\omega}_{2}\right)+\left|\boldsymbol{v}_{1} \boldsymbol{\omega}_{1} \boldsymbol{\omega}_{2}\right|\left\|\boldsymbol{\omega}_{2}\right\|^{2}\right)\left\|\boldsymbol{\omega}_{3} \times \boldsymbol{\omega}_{2}\right\|^{2} \\
& \frac{-\left(\left|\boldsymbol{v}_{2} \boldsymbol{\omega}_{2} \boldsymbol{\omega}_{3}\right|\left(\boldsymbol{\omega}_{3} \cdot \boldsymbol{\omega}_{2}\right)+\left|\boldsymbol{v}_{3} \boldsymbol{\omega}_{3} \boldsymbol{\omega}_{2}\right|\left\|\boldsymbol{\omega}_{2}\right\|^{2}\right)\left\|\boldsymbol{\omega}_{1} \times \boldsymbol{\omega}_{2}\right\|^{2}}{\left\|\boldsymbol{\omega}_{2}\right\|\left\|\boldsymbol{\omega}_{3} \times \boldsymbol{\omega}_{2}\right\|^{2}\left\|\boldsymbol{\omega}_{1} \times \boldsymbol{\omega}_{2}\right\|^{2}} .
\end{aligned}
$$

Note that under our assumption above, this quantity is well-defined. Similarly, provided the axes of screws 1 and 3 are also non-parallel, offsets 1 and 3 can be written as:

$$
\begin{aligned}
& d_{1}=\left(\left|\boldsymbol{v}_{1} \boldsymbol{\omega}_{1} \boldsymbol{\omega}_{3}\right|\left(\boldsymbol{\omega}_{3} \cdot \boldsymbol{\omega}_{1}\right)+\left|\boldsymbol{v}_{3} \boldsymbol{\omega}_{3} \boldsymbol{\omega}_{1}\right|\left\|\boldsymbol{\omega}_{1}\right\|^{2}\right)\left\|\boldsymbol{\omega}_{2} \times \boldsymbol{\omega}_{1}\right\|^{2} \\
& \frac{-\left(\left|\boldsymbol{v}_{1} \boldsymbol{\omega}_{1} \boldsymbol{\omega}_{2}\right|\left(\boldsymbol{\omega}_{2} \cdot \boldsymbol{\omega}_{1}\right)+\left|\boldsymbol{v}_{2} \boldsymbol{\omega}_{2} \boldsymbol{\omega}_{1}\right|\left\|\boldsymbol{\omega}_{1}\right\|^{2}\right)\left\|\boldsymbol{\omega}_{3} \times \boldsymbol{\omega}_{1}\right\|^{2}}{\left\|\boldsymbol{\omega}_{1}\right\|\left\|\boldsymbol{\omega}_{2} \times \boldsymbol{\omega}_{1}\right\|^{2}\left\|\boldsymbol{\omega}_{3} \times \boldsymbol{\omega}_{1}\right\|^{2}}, \\
& d_{3}=\left(\left|\boldsymbol{v}_{3} \boldsymbol{\omega}_{3} \boldsymbol{\omega}_{2}\right|\left(\boldsymbol{\omega}_{2} \cdot \boldsymbol{\omega}_{3}\right)+\left|\boldsymbol{v}_{2} \boldsymbol{\omega}_{2} \boldsymbol{\omega}_{3}\right|\left\|\boldsymbol{\omega}_{3}\right\|^{2}\right)\left\|\boldsymbol{\omega}_{1} \times \boldsymbol{\omega}_{3}\right\|^{2} \\
& \frac{-\left(\left|\boldsymbol{v}_{3} \boldsymbol{\omega}_{3} \boldsymbol{\omega}_{1}\right|\left(\boldsymbol{\omega}_{1} \cdot \boldsymbol{\omega}_{3}\right)+\left|\boldsymbol{v}_{1} \boldsymbol{\omega}_{1} \boldsymbol{\omega}_{3}\right|\left\|\boldsymbol{\omega}_{3}\right\|^{2}\right)\left\|\boldsymbol{\omega}_{2} \times \boldsymbol{\omega}_{3}\right\|^{2}}{\left\|\boldsymbol{\omega}_{3}\right\|\left\|\boldsymbol{\omega}_{1} \times \boldsymbol{\omega}_{3}\right\|^{2}\left\|\boldsymbol{\omega}_{2} \times \boldsymbol{\omega}_{3}\right\|^{2}}
\end{aligned}
$$

The numerator $\Omega_{2}$ of the offset $d_{2}$ can be written as a determinant of

\footnotetext{
${ }^{2}$ We omit the modulus sign to simplify notation. However, offset is positive.
} 
$4 \times 4$-matrix as following

$$
\Omega_{2}=-\frac{1}{2}\left|\begin{array}{cccc}
\left|\boldsymbol{v}_{1} \boldsymbol{\omega}_{1} \boldsymbol{\omega}_{2}\right| & -\left(\boldsymbol{\omega}_{1} \cdot \boldsymbol{\omega}_{2}\right) & \left(\boldsymbol{\omega}_{1} \cdot \boldsymbol{\omega}_{2}\right) & \left(\boldsymbol{\omega}_{1} \cdot \boldsymbol{\omega}_{1}\right) \\
\left|\boldsymbol{v}_{2} \boldsymbol{\omega}_{2} \boldsymbol{\omega}_{1}\right| & \left(\boldsymbol{\omega}_{2} \cdot \boldsymbol{\omega}_{2}\right) & -\left(\boldsymbol{\omega}_{2} \cdot \boldsymbol{\omega}_{2}\right) & -\left(\boldsymbol{\omega}_{1} \cdot \boldsymbol{\omega}_{2}\right) \\
\left|\boldsymbol{v}_{2} \boldsymbol{\omega}_{2} \boldsymbol{\omega}_{3}\right| & -\left(\boldsymbol{\omega}_{2} \cdot \boldsymbol{\omega}_{2}\right) & -\left(\boldsymbol{\omega}_{2} \cdot \boldsymbol{\omega}_{2}\right) & -\left(\boldsymbol{\omega}_{2} \cdot \boldsymbol{\omega}_{3}\right) \\
\left|\boldsymbol{v}_{3} \boldsymbol{\omega}_{3} \boldsymbol{\omega}_{2}\right| & \left(\boldsymbol{\omega}_{2} \cdot \boldsymbol{\omega}_{3}\right) & \left(\boldsymbol{\omega}_{2} \cdot \boldsymbol{\omega}_{3}\right) & \left(\boldsymbol{\omega}_{3} \cdot \boldsymbol{\omega}_{3}\right)
\end{array}\right|
$$

Similar expression can be obtained for the numerator of offsets $d_{1}$ and $d_{3}$ :

$$
\begin{array}{r}
\Omega_{1}=-\frac{1}{2}\left|\begin{array}{cccc}
\left|\boldsymbol{v}_{3} \boldsymbol{\omega}_{3} \boldsymbol{\omega}_{1}\right| & -\left(\boldsymbol{\omega}_{1} \cdot \boldsymbol{\omega}_{3}\right) & \left(\boldsymbol{\omega}_{1} \cdot \boldsymbol{\omega}_{3}\right) & \left(\boldsymbol{\omega}_{3} \cdot \boldsymbol{\omega}_{3}\right) \\
\left|\boldsymbol{v}_{1} \boldsymbol{\omega}_{1} \boldsymbol{\omega}_{3}\right| & \left(\boldsymbol{\omega}_{1} \cdot \boldsymbol{\omega}_{1}\right) & -\left(\boldsymbol{\omega}_{1} \cdot \boldsymbol{\omega}_{1}\right) & -\left(\boldsymbol{\omega}_{1} \cdot \boldsymbol{\omega}_{3}\right) \\
\left|\boldsymbol{v}_{1} \boldsymbol{\omega}_{1} \boldsymbol{\omega}_{2}\right| & -\left(\boldsymbol{\omega}_{1} \cdot \boldsymbol{\omega}_{1}\right) & -\left(\boldsymbol{\omega}_{1} \cdot \boldsymbol{\omega}_{1}\right) & -\left(\boldsymbol{\omega}_{1} \cdot \boldsymbol{\omega}_{2}\right) \\
\left|\boldsymbol{v}_{2} \boldsymbol{\omega}_{2} \boldsymbol{\omega}_{1}\right| & \left(\boldsymbol{\omega}_{1} \cdot \boldsymbol{\omega}_{2}\right) & \left(\boldsymbol{\omega}_{1} \cdot \boldsymbol{\omega}_{2}\right) & \left(\boldsymbol{\omega}_{2} \cdot \boldsymbol{\omega}_{2}\right)
\end{array}\right| \\
\Omega_{3}=-\frac{1}{2}\left|\begin{array}{llll}
\left|\boldsymbol{v}_{2} \boldsymbol{\omega}_{2} \boldsymbol{\omega}_{3}\right| & -\left(\boldsymbol{\omega}_{2} \cdot \boldsymbol{\omega}_{3}\right) & \left(\boldsymbol{\omega}_{2} \cdot \boldsymbol{\omega}_{3}\right) & \left(\boldsymbol{\omega}_{2} \cdot \boldsymbol{\omega}_{2}\right) \\
\left|\boldsymbol{v}_{3} \boldsymbol{\omega}_{3} \boldsymbol{\omega}_{2}\right| & \left(\boldsymbol{\omega}_{3} \cdot \boldsymbol{\omega}_{3}\right) & -\left(\boldsymbol{\omega}_{3} \cdot \boldsymbol{\omega}_{3}\right) & -\left(\boldsymbol{\omega}_{2} \cdot \boldsymbol{\omega}_{3}\right) \\
\left|\boldsymbol{v}_{3} \boldsymbol{\omega}_{3} \boldsymbol{\omega}_{1}\right| & -\left(\boldsymbol{\omega}_{3} \cdot \boldsymbol{\omega}_{3}\right) & -\left(\boldsymbol{\omega}_{3} \cdot \boldsymbol{\omega}_{3}\right) & -\left(\boldsymbol{\omega}_{1} \cdot \boldsymbol{\omega}_{3}\right) \\
\left|\boldsymbol{v}_{1} \boldsymbol{\omega}_{1} \boldsymbol{\omega}_{3}\right| & \left(\boldsymbol{\omega}_{1} \cdot \boldsymbol{\omega}_{3}\right) & \left(\boldsymbol{\omega}_{1} \cdot \boldsymbol{\omega}_{3}\right) & \left(\boldsymbol{\omega}_{1} \cdot \boldsymbol{\omega}_{1}\right)
\end{array}\right| .
\end{array}
$$

The offsets are invariant under the adjoint action of $S E(3)$ acting on triple screws, we can verify that by Maple. It is clear that the denominator of any one of them can be written in terms of the invariants of adjoint action of $S E(3)$ acting on triple screws. However, it is not clear that the numerator can be written in terms of those vector invariants, since we have determinants, for instance $\left|v_{2} \omega_{2} \omega_{1}\right|,\left|v_{1} \omega_{1} \omega_{2}\right|$ and $\left|v_{3} \omega_{3} \omega_{1}\right|$, which are not among the fourteen vector invariants of $S E(3)$ acting on triple screws. In fact the indices of the vectors in any of those determinants do not belong to the even permutation of three vectors. While those determinants are linear in the $v$ 's there is no clear evidence that they can be written in terms of the invariants in Theorem 6.3 .1 . 
Lemma 8.4.1. The product of the invariant $\Omega_{2}$ and $I_{123}$ can be expressed as a polynomial in terms of vector invariants of the adjoint action of SE(3).

Proof.

$$
\begin{aligned}
\Omega_{2} I_{123} & =\left|\boldsymbol{v}_{2} \boldsymbol{\omega}_{2} \boldsymbol{\omega}_{1}\right|\left|\boldsymbol{\omega}_{1} \boldsymbol{\omega}_{2} \boldsymbol{\omega}_{3}\right|\left(\boldsymbol{\omega}_{1} \cdot \boldsymbol{\omega}_{2}\right)\left\|\boldsymbol{\omega}_{3} \times \boldsymbol{\omega}_{2}\right\|^{2} \\
& +\left|\boldsymbol{v}_{1} \boldsymbol{\omega}_{1} \boldsymbol{\omega}_{2}\right|\left|\boldsymbol{\omega}_{1} \boldsymbol{\omega}_{2} \boldsymbol{\omega}_{3}\right|\left\|\boldsymbol{\omega}_{2}\right\|^{2}\left\|\boldsymbol{\omega}_{3} \times \boldsymbol{\omega}_{2}\right\|^{2} \\
& -\left|\boldsymbol{v}_{2} \boldsymbol{\omega}_{2} \boldsymbol{\omega}_{3}\right|\left|\boldsymbol{\omega}_{1} \boldsymbol{\omega}_{2} \boldsymbol{\omega}_{3}\right|\left(\boldsymbol{\omega}_{3} \cdot \boldsymbol{\omega}_{2}\right)\left\|\boldsymbol{\omega}_{1} \times \boldsymbol{\omega}_{2}\right\|^{2} \\
& -\left|\boldsymbol{v}_{3} \boldsymbol{\omega}_{3} \boldsymbol{\omega}_{2}\right|\left|\boldsymbol{\omega}_{1} \boldsymbol{\omega}_{2} \boldsymbol{\omega}_{3}\right|\left\|\boldsymbol{\omega}_{2}\right\|^{2}\left\|\boldsymbol{\omega}_{1} \times \boldsymbol{\omega}_{2}\right\|^{2}
\end{aligned}
$$

We note that each term has a product of two determinants so we have to expand those determinants as follows:

$$
\left|\boldsymbol{v}_{2} \boldsymbol{\omega}_{2} \boldsymbol{\omega}_{1}\right|\left|\boldsymbol{\omega}_{1} \boldsymbol{\omega}_{2} \boldsymbol{\omega}_{3}\right|=\left|\begin{array}{lll}
\left(\boldsymbol{v}_{2} \cdot \boldsymbol{\omega}_{1}\right) & \left(\boldsymbol{v}_{2} \cdot \boldsymbol{\omega}_{2}\right) & \left(\boldsymbol{v}_{2} \cdot \boldsymbol{\omega}_{3}\right) \\
\left(\boldsymbol{\omega}_{2} \cdot \boldsymbol{\omega}_{1}\right) & \left(\boldsymbol{\omega}_{2} \cdot \boldsymbol{\omega}_{2}\right) & \left(\boldsymbol{\omega}_{2} \cdot \boldsymbol{\omega}_{3}\right) \\
\left(\boldsymbol{\omega}_{1} \cdot \boldsymbol{\omega}_{1}\right) & \left(\boldsymbol{\omega}_{1} \cdot \boldsymbol{\omega}_{2}\right) & \left(\boldsymbol{\omega}_{1} \cdot \boldsymbol{\omega}_{3}\right)
\end{array}\right|
$$

We can express this in terms of known vector invariants of the adjoint action of $S E(3)$ in Theorem 6.3.1 in order to make the calculation easier.

$$
\begin{aligned}
& =\left|\begin{array}{ccc}
\left(\boldsymbol{v}_{2} \cdot \boldsymbol{\omega}_{1}\right) & \hat{I}_{22} & \left(\boldsymbol{v}_{2} \cdot \boldsymbol{\omega}_{3}\right) \\
I_{12} & I_{22} & I_{23} \\
I_{11} & I_{12} & I_{13}
\end{array}\right| \\
= & \left(\boldsymbol{v}_{2} \cdot \boldsymbol{\omega}_{1}\right) I_{22} I_{13}-\left(\boldsymbol{v}_{2} \cdot \boldsymbol{\omega}_{1}\right) I_{23} I_{12}-I_{12} \hat{I}_{22} I_{13} \\
+ & I_{12} I_{12}\left(\boldsymbol{v}_{2} \cdot \boldsymbol{\omega}_{3}\right)+I_{11} \hat{I}_{22} I_{23}-I_{11} I_{22}\left(\boldsymbol{v}_{2} \cdot \boldsymbol{\omega}_{3}\right) .
\end{aligned}
$$


Similarly,

$$
\begin{aligned}
\left|\boldsymbol{v}_{1} \boldsymbol{\omega}_{1} \boldsymbol{\omega}_{2}\right|\left|\boldsymbol{\omega}_{1} \boldsymbol{\omega}_{2} \boldsymbol{\omega}_{3}\right| & =\hat{I}_{11} I_{12} I_{23}-\hat{I}_{11} I_{13} I_{22}-I_{11} I_{23}\left(\boldsymbol{v}_{1} \cdot \boldsymbol{\omega}_{2}\right) \\
& +I_{11} I_{22}\left(\boldsymbol{v}_{1} \cdot \boldsymbol{\omega}_{3}\right)+I_{12} I_{13}\left(\boldsymbol{v}_{1} \cdot \boldsymbol{\omega}_{2}\right)-I_{12}^{2}\left(\boldsymbol{v}_{1} \cdot \boldsymbol{\omega}_{3}\right) \\
\left|\boldsymbol{v}_{2} \boldsymbol{\omega}_{2} \boldsymbol{\omega}_{3}\right|\left|\boldsymbol{\omega}_{1} \boldsymbol{\omega}_{2} \boldsymbol{\omega}_{3}\right| & =\left(\boldsymbol{v}_{2} \cdot \boldsymbol{\omega}_{1}\right) I_{22} I_{33}-\left(\boldsymbol{v}_{2} \cdot \boldsymbol{\omega}_{1}\right) I_{23}^{2}-I_{12} \hat{I}_{22} I_{33} \\
& +I_{12} I_{23}\left(\boldsymbol{v}_{2} \cdot \boldsymbol{\omega}_{3}\right)+I_{13} \hat{I}_{22} I_{23}-I_{13} I_{22}\left(\boldsymbol{v}_{2} \cdot \boldsymbol{\omega}_{3}\right) \\
\left|\boldsymbol{v}_{3} \boldsymbol{\omega}_{3} \boldsymbol{\omega}_{2}\right|\left|\boldsymbol{\omega}_{1} \boldsymbol{\omega}_{2} \boldsymbol{\omega}_{3}\right| & =\left(\boldsymbol{v}_{3} \cdot \boldsymbol{\omega}_{1}\right) I_{23}^{2}-\left(\boldsymbol{v}_{3} \cdot \boldsymbol{\omega}_{1}\right) I_{33} I_{22}-I_{13} I_{23}\left(\boldsymbol{v}_{3} \cdot \boldsymbol{\omega}_{2}\right) \\
& +I_{13} I_{22} \hat{I}_{33}+I_{12} I_{33}\left(\boldsymbol{v}_{3} \cdot \boldsymbol{\omega}_{2}\right)-I_{12} I_{23} \hat{I}_{33}
\end{aligned}
$$

By substituting the product of two determinants of each term in 8.31 by 8.33 , 8.34, 8.35 and 8.36) respectively, and write $\left\|\boldsymbol{\omega}_{1} \times \boldsymbol{\omega}_{2}\right\|^{2}$, and $\left\|\boldsymbol{\omega}_{2} \times \boldsymbol{\omega}_{3}\right\|^{2}$ in terms of known vector invariants as $I_{11} I_{22}-I_{12}^{2}$, and $I_{22} I_{33}-I_{23}^{2}$ respectively, and sorting the indices of invariants in ascending order and treating brackets as coefficients. Then we get:

$$
\begin{aligned}
\Omega_{2} I_{123} & =\left(\boldsymbol{v}_{2} \cdot \boldsymbol{\omega}_{1}\right) I_{12} I_{13} I_{22}^{2} I_{33}-\left(\boldsymbol{v}_{2} \cdot \boldsymbol{\omega}_{1}\right) I_{12}^{2} I_{22} I_{23} I_{33}-I_{12}^{2} I_{13} I_{22} \hat{I}_{22} I_{33} \\
& +\left(\boldsymbol{v}_{2} \cdot \boldsymbol{\omega}_{3}\right) I_{12}^{3} I_{22} I_{33}+I_{11} I_{12} I_{22} \hat{I}_{22} I_{23} I_{33}-\left(\boldsymbol{v}_{2} \cdot \boldsymbol{\omega}_{3}\right) I_{11} I_{12} I_{22}^{2} I_{33} \\
& -\left(\boldsymbol{v}_{2} \cdot \boldsymbol{\omega}_{1}\right) I_{12} I_{13} I_{22} I_{23}^{2}+\left(\boldsymbol{v}_{2} \cdot \boldsymbol{\omega}_{1}\right) I_{12}^{2} I_{23}^{3}+I_{12}^{2} I_{13} \hat{I}_{22} I_{23}^{2} \\
& -\left(\boldsymbol{v}_{2} \cdot \boldsymbol{\omega}_{3}\right) I_{12}^{3} I_{23}^{2}-I_{11} I_{12} \hat{I}_{22} I_{23}^{3}+\left(\boldsymbol{v}_{2} \cdot \boldsymbol{\omega}_{3}\right) I_{11} I_{12} I_{22} I_{23}^{2} \\
& +\hat{I}_{11} I_{12} I_{22}^{2} I_{23} I_{33}-\hat{I}_{11} I_{13} I_{22}^{3} I_{33}-\left(\boldsymbol{v}_{1} \cdot \boldsymbol{\omega}_{2}\right) I_{11} I_{22}^{2} I_{23} I_{33} \\
& +\left(\boldsymbol{v}_{1} \cdot \boldsymbol{\omega}_{3}\right) I_{11} I_{22}^{3} I_{33}+\left(\boldsymbol{v}_{1} \cdot \boldsymbol{\omega}_{2}\right) I_{12} I_{13} I_{22}^{2} I_{33}-\left(\boldsymbol{v}_{1} \cdot \boldsymbol{\omega}_{3}\right) I_{12}^{2} I_{22}^{2} I_{33} \\
& -\hat{I}_{11} I_{12} I_{22} I_{23}^{3}+\hat{I}_{11} I_{13} I_{22}^{2} I_{23}^{2}+\left(\boldsymbol{v}_{1} \cdot \boldsymbol{\omega}_{2}\right) I_{11} I_{22} I_{23}^{3} \\
& -\left(\boldsymbol{v}_{1} \cdot \boldsymbol{\omega}_{3}\right) I_{11} I_{22}^{2} I_{23}^{2}-\left(\boldsymbol{v}_{1} \cdot \boldsymbol{\omega}_{2}\right) I_{12} I_{13} I_{22} I_{23}^{2}+\left(\boldsymbol{v}_{1} \cdot \boldsymbol{\omega}_{3}\right) I_{12}^{2} I_{22} I_{23}^{2}
\end{aligned}
$$




$$
\begin{aligned}
& -\left(\boldsymbol{v}_{2} \cdot \boldsymbol{\omega}_{1}\right) I_{11} I_{22}^{2} I_{23} I_{33}+\left(\boldsymbol{v}_{2} \cdot \boldsymbol{\omega}_{1}\right) I_{11} I_{22} I_{23}^{3}+I_{11} I_{12} I_{22} \hat{I}_{22} I_{23} I_{33} \\
& -\left(\boldsymbol{v}_{2} \cdot \boldsymbol{\omega}_{3}\right) I_{11} I_{12} I_{22} I_{23}^{2}-I_{11} I_{13} I_{22} \hat{I}_{22} I_{23}^{2}+\left(\boldsymbol{v}_{2} \cdot \boldsymbol{\omega}_{3}\right) I_{11} I_{13} I_{22}^{2} I_{23} \\
& +\left(\boldsymbol{v}_{2} \cdot \boldsymbol{\omega}_{1}\right) I_{12}^{2} I_{22} I_{23} I_{33}-\left(\boldsymbol{v}_{2} \cdot \boldsymbol{\omega}_{1}\right) I_{12}^{2} I_{23}^{3}-I_{12}^{3} I_{23} \hat{I}_{22} I_{33} \\
& +\left(\boldsymbol{v}_{2} \cdot \boldsymbol{\omega}_{3}\right) I_{12}^{3} I_{23}^{2}+I_{12}^{2} I_{13} \hat{I}_{22} I_{23}^{2}-\left(\boldsymbol{v}_{2} \cdot \boldsymbol{\omega}_{3}\right) I_{12}^{2} I_{13} I_{22} I_{23} \\
& -\left(\boldsymbol{v}_{3} \cdot \boldsymbol{\omega}_{1}\right) I_{11} I_{22}^{2} I_{23}^{2}+\left(\boldsymbol{v}_{3} \cdot \boldsymbol{\omega}_{1}\right) I_{11} I_{22}^{3} I_{33}+\left(\boldsymbol{v}_{3} \cdot \boldsymbol{\omega}_{2}\right) I_{11} I_{13} I_{23} I_{22}^{2} \\
& -I_{11} I_{13} I_{22}^{3} \hat{I}_{33}-\left(\boldsymbol{v}_{3} \cdot \boldsymbol{\omega}_{2}\right) I_{11} I_{12} I_{22}^{2} I_{33}+I_{11} I_{12} I_{22}^{2} I_{23} \hat{I}_{33} \\
& +\left(\boldsymbol{v}_{3} \cdot \boldsymbol{\omega}_{1}\right) I_{12}^{2} I_{22} I_{23}^{2}-\left(\boldsymbol{v}_{3} \cdot \boldsymbol{\omega}_{1}\right) I_{12}^{2} I_{22}^{2} I_{33}-\left(\boldsymbol{v}_{3} \cdot \boldsymbol{\omega}_{2}\right) I_{12}^{2} I_{13} I_{22} I_{23} \\
& +I_{12}^{2} I_{13} I_{22}^{2} \hat{I}_{33}+\left(\boldsymbol{v}_{3} \cdot \boldsymbol{\omega}_{2}\right) I_{12}^{3} I_{22} I_{33}-I_{12}^{3} I_{22} I_{23} \hat{I}_{33} .
\end{aligned}
$$

By combining the terms together we get

$$
\begin{aligned}
& \Omega_{2} I_{123}=\left(\boldsymbol{v}_{1} \cdot \boldsymbol{\omega}_{2}+\boldsymbol{v}_{2} \cdot \boldsymbol{\omega}_{1}\right) I_{11} I_{22} I_{23}^{3}+\left(\boldsymbol{v}_{2} \cdot \boldsymbol{\omega}_{1}+\boldsymbol{v}_{1} \cdot \boldsymbol{\omega}_{2}\right) I_{12} I_{13} I_{22}^{2} I_{33} \\
& -\left(\boldsymbol{v}_{2} \cdot \boldsymbol{\omega}_{1}+\boldsymbol{v}_{1} \cdot \boldsymbol{\omega}_{2}\right) I_{12} I_{13} I_{22} I_{23}^{2}-\left(\boldsymbol{v}_{1} \cdot \boldsymbol{\omega}_{2}+\boldsymbol{v}_{2} \cdot \boldsymbol{\omega}_{1}\right) I_{11} I_{22}^{2} I_{23} I_{33} \\
& +\left(\boldsymbol{v}_{2} \cdot \boldsymbol{\omega}_{3}+\boldsymbol{v}_{3} \cdot \boldsymbol{\omega}_{2}\right) I_{12}^{3} I_{22} I_{33}+\left(\boldsymbol{v}_{2} \cdot \boldsymbol{\omega}_{3}+\boldsymbol{v}_{3} \cdot \boldsymbol{\omega}_{2}\right) I_{11} I_{13} I_{22}^{2} I_{23} \\
& -\left(\boldsymbol{v}_{3} \cdot \boldsymbol{\omega}_{2}+\boldsymbol{v}_{2} \cdot \boldsymbol{\omega}_{3}\right) I_{12}^{2} I_{13} I_{22} I_{23}-\left(\boldsymbol{v}_{2} \cdot \boldsymbol{\omega}_{3}+\boldsymbol{v}_{3} \cdot \boldsymbol{\omega}_{2}\right) I_{11} I_{12} I_{22}^{2} I_{33} \\
& +\left(\boldsymbol{v}_{1} \cdot \boldsymbol{\omega}_{3}+\boldsymbol{v}_{3} \cdot \boldsymbol{\omega}_{1}\right) I_{11} I_{22}^{3} I_{33}-\left(\boldsymbol{v}_{1} \cdot \boldsymbol{\omega}_{3}+\boldsymbol{v}_{3} \cdot \boldsymbol{\omega}_{1}\right) I_{12}^{2} I_{22}^{2} I_{33} \\
& -\left(\boldsymbol{v}_{1} \cdot \boldsymbol{\omega}_{3}+\boldsymbol{v}_{3} \cdot \boldsymbol{\omega}_{1}\right) I_{11} I_{22}^{2} I_{23}^{2}+\left(\boldsymbol{v}_{1} \cdot \boldsymbol{\omega}_{3}+\boldsymbol{v}_{3} \cdot \boldsymbol{\omega}_{1}\right) I_{12}^{2} I_{22} I_{23}^{2} \\
& +\hat{I}_{11} I_{12} I_{22}^{2} I_{23} I_{33}-\hat{I}_{11} I_{13} I_{22}^{3} I_{33}-I_{12}^{2} I_{13} I_{22} \hat{I}_{22} I_{33}+I_{12}^{2} I_{13} \hat{I}_{22} I_{23}^{2} \\
& -I_{11} I_{12} \hat{I}_{22} I_{23}^{3}-I_{12}^{3} I_{22} I_{23} \hat{I}_{33}-\hat{I}_{11} I_{12} I_{22} I_{23}^{3}+\hat{I}_{11} I_{13} I_{22}^{2} I_{23}^{2} \\
& +I_{11} I_{12} I_{22} \hat{I}_{22} I_{23} I_{33}-I_{11} I_{13} I_{22} \hat{I}_{22} I_{23}^{2}+I_{12}^{2} I_{13} \hat{I}_{22} I_{23}^{2}-I_{12}^{3} I_{23} \hat{I}_{22} I_{33} \\
& -I_{11} I_{13} I_{22}^{3} \hat{I}_{33}+I_{11} I_{12} I_{22}^{2} I_{23} \hat{I}_{33}+I_{12}^{2} I_{13} I_{22}^{2} \hat{I}_{33}+I_{11} I_{12} I_{22} \hat{I}_{22} I_{23} I_{33} \\
& +\left(\boldsymbol{v}_{2} \cdot \boldsymbol{w}_{3}\right) I_{12}^{3} I_{23}^{2}-\left(\boldsymbol{v}_{2} \cdot \boldsymbol{w}_{3}\right) I_{12}^{3} I_{23}^{2}+\left(\boldsymbol{v}_{2} \cdot \boldsymbol{\omega}_{3}\right) I_{11} I_{12} I_{22} I_{23}^{2} \\
& -\underline{\left(\boldsymbol{v}_{2} \cdot \boldsymbol{\omega}_{3}\right) I_{11} I_{12} I_{22} I_{23}^{2}}-\underline{\left(\boldsymbol{v}_{2} \cdot \boldsymbol{\omega}_{1}\right) I_{12}^{2} I_{23}^{3}}+\underline{\left(\boldsymbol{v}_{2} \cdot \boldsymbol{\omega}_{1}\right) I_{12}^{2} I_{23}^{3}} \\
& -\underline{\left(\boldsymbol{v}_{2} \cdot \boldsymbol{\omega}_{1}\right) I_{12}^{2} I_{22} I_{23} I_{33}}+\underline{\left(\boldsymbol{v}_{2} \cdot \boldsymbol{\omega}_{1}\right) I_{12}^{2} I_{22} I_{23} I_{33}} .
\end{aligned}
$$


Remember $\boldsymbol{v}_{1} \cdot \boldsymbol{\omega}_{2}+\boldsymbol{v}_{2} \cdot \boldsymbol{\omega}_{1}, \boldsymbol{v}_{1} \cdot \boldsymbol{\omega}_{3}+\boldsymbol{v}_{3} \cdot \boldsymbol{\omega}_{1}$ and $\boldsymbol{v}_{3} \cdot \boldsymbol{\omega}_{2}+\boldsymbol{v}_{2} \cdot \boldsymbol{\omega}_{3}$ are equal to invariants $\hat{I}_{12}, \hat{I}_{13}$ and $\hat{I}_{23}$ respectively. Hence

$$
\begin{aligned}
\Omega_{2} I_{123} & =I_{11} \hat{I}_{12} I_{22} I_{23}^{3}+\hat{I}_{12} I_{12} I_{13} I_{22}^{2} I_{33}-\hat{I}_{12} I_{12} I_{13} I_{22} I_{23}^{2}-I_{11} \hat{I}_{12} I_{22}^{2} I_{23} I_{33} \\
& +I_{12}^{3} I_{22} \hat{I}_{23} I_{33}+I_{11} I_{13} I_{22}^{2} I_{23} \hat{I}_{23}-I_{12}^{2} I_{13} I_{22} I_{23} \hat{I}_{23}-I_{11} I_{12} I_{22}^{2} \hat{I}_{23} I_{33} \\
& +I_{11} \hat{I}_{13} I_{22}^{3} I_{33}-I_{12}^{2} \hat{I}_{13} I_{22}^{2} I_{33}-I_{11} \hat{I}_{13} I_{22}^{2} I_{23}^{2}+I_{12}^{2} \hat{I}_{13} I_{22} I_{23}^{2} \\
& +\hat{I}_{11} I_{12} I_{22}^{2} I_{23} I_{33}-\hat{I}_{11} I_{13} I_{22}^{3} I_{33}-I_{12}^{2} I_{13} I_{22} \hat{I}_{22} I_{33}+2 I_{12}^{2} I_{13} \hat{I}_{22} I_{23}^{2} \\
& -I_{11} I_{12} \hat{I}_{22} I_{23}^{3}-I_{12}^{3} I_{22} I_{23} \hat{I}_{33}-\hat{I}_{11} I_{12} I_{22} I_{23}^{3}+\hat{I}_{11} I_{13} I_{22}^{2} I_{23}^{2} \\
& +2 I_{11} I_{12} I_{22} \hat{I}_{22} I_{23} I_{33}-I_{11} I_{13} I_{22} \hat{I}_{22} I_{23}^{2}-I_{12}^{3} I_{23} \hat{I}_{22} I_{33}-I_{11} I_{13} I_{22}^{3} \hat{I}_{33} \\
& +I_{11} I_{12} I_{22}^{2} I_{23} \hat{I}_{33}+I_{12}^{2} I_{13} I_{22}^{2} \hat{I}_{33} .
\end{aligned}
$$

Theorem 8.4.2. The numerator $\Omega_{2}$ of $d_{2}$ can be expressed as a polynomial in terms of vector invariants of the adjoint action of $S E(3)$ acting on triple screws.

Proof. Lemma 8.4.1 tells us there is an invariant expression for the product of the offset numerator $\Omega_{2}$ and vector invariant $I_{123}$, while Result 6.6 also tells us that there is an expression for the product vector invariants $I_{123}$ and $\hat{I}_{123}$. Hence we can write $\Omega_{2}$ as a combination of these syzygies with the other algebraically independent vector invariants of the adjoint action of $S E(3)$ acting on triple screws.

If we add $I_{22} I_{123} \hat{I}_{123}\left(I_{13} I_{22}-I_{12} I_{23}\right)$ to both sides of Equation 8.39 , then we get

$$
\begin{aligned}
& \Omega_{2} I_{123}+I_{22} I_{123} \hat{I}_{123}\left(I_{13} I_{22}-I_{12} I_{23}\right)=I_{11} I_{12} I_{22} \hat{I}_{22} I_{23} I_{33}+2 I_{12}^{2} I_{13} \hat{I}_{22} I_{23}^{2} \\
& -I_{11} I_{12} \hat{I}_{22} I_{23}^{3}-I_{12}^{3} \hat{I}_{22} I_{23} I_{33}+I_{12} I_{13}^{2} I_{22} \hat{I}_{22} I_{23}+I_{11} I_{13} I_{22}^{2} \hat{I}_{22} I_{33}-I_{11} I_{13} I_{22} \hat{I}_{22} I_{23}^{2}
\end{aligned}
$$




$$
\begin{aligned}
& -I_{12}^{2} I_{13} I_{22} \hat{I}_{22} I_{33}+2 I_{12} I_{13}^{2} I_{22} \hat{I}_{22} I_{23}-I_{13}^{3} I_{22}^{2} \hat{I}_{22}+I_{11} \hat{I}_{13} I_{22}^{3} I_{33}-I_{11} \hat{I}_{13} I_{23}^{2} I_{22}^{2} \\
& -I_{12}^{2} \hat{I}_{13} I_{22}^{2} I_{33}+2 I_{12} I_{13} \hat{I}_{13} I_{22}^{2} I_{23}-I_{13}^{2} \hat{I}_{13} I_{22}^{3}-I_{11} I_{12} I_{22}^{2} \hat{I}_{23} I_{33}+I_{11} I_{12} I_{22} I_{23}^{2} \hat{I}_{23} \\
& +I_{12}^{3} I_{22} \hat{I}_{23} I_{33}-2 I_{12}^{2} I_{13} I_{22} I_{23} \hat{I}_{23}+I_{12} I_{13}^{2} I_{22}^{2} \hat{I}_{23}-I_{11} \hat{I}_{12} I_{22}^{2} I_{23} I_{33} \\
& +I_{11} \hat{I}_{12} I_{22} I_{23}^{3}+I_{12}^{2} \hat{I}_{12} I_{22} I_{23} I_{33}-2 I_{12} \hat{I}_{12} I_{13} I_{22} I_{23}^{2}+\hat{I}_{12} I_{13}^{2} I_{22}^{2} I_{23}
\end{aligned}
$$

The terms on the right hand side can be collected as follows:

$$
\begin{aligned}
& =I_{12} \hat{I}_{22} I_{23}\left(I_{11} I_{22} I_{33}+2 I_{12} I_{13} I_{23}-I_{11} I_{23}^{2}-I_{12}^{2} I_{33}+I_{13}^{2} I_{22}\right) \\
& +I_{13} I_{22} \hat{I}_{22}\left(I_{11} I_{22} I_{33}-I_{11} I_{23}^{2}-I_{12}^{2} I_{33}+2 I_{12} I_{13} I_{23}-I_{13}^{2} I_{22}\right) \\
& +\hat{I}_{13} I_{22}^{2}\left(I_{11} I_{22} I_{33}-I_{11} I_{23}^{2}-I_{12}^{2} I_{33}+2 I_{12} I_{13} I_{23}-I_{13}^{2} I_{22}\right) \\
& -I_{12} I_{22} \hat{I}_{23}\left(I_{11} I_{22} I_{33}-I_{11} I_{23}^{2}-I_{12}^{2} I_{33}+2 I_{12} I_{13} I_{23}-I_{13}^{2} I_{22}\right) \\
& -\hat{I}_{12} I_{22} I_{23}\left(I_{11} I_{22} I_{33}-I_{11} I_{23}^{2}-I_{12}^{2} I_{33}+2 I_{12} I_{13} I_{23}-I_{13}^{2} I_{22}\right) \\
& =\left(I_{12} \hat{I}_{22} I_{23}+I_{13} I_{22} \hat{I}_{22}+\hat{I}_{13} I_{22}^{2}-I_{12} I_{22} \hat{I}_{23}-\hat{I}_{12} I_{22} I_{23}\right)\left(I_{11} I_{22} I_{33}\right. \\
& \left.\quad-\quad I_{11} I_{23}^{2}-I_{12}^{2} I_{33}+2 I_{12} I_{13} I_{23}-I_{13}^{2} I_{22}\right) .
\end{aligned}
$$

From 6.5 we can replace the second factor by $I_{123}^{2}$, so that:

$$
\begin{aligned}
\Omega_{2} I_{123} & +I_{22} I_{123} \hat{I}_{123}\left(I_{13} I_{22}-I_{12} I_{23}\right)=\left(I_{12} \hat{I}_{22} I_{23}\right. \\
& \left.+I_{13} I_{22} \hat{I}_{22}+\hat{I}_{13} I_{22}^{2}-I_{12} I_{22} \hat{I}_{23}-\hat{I}_{12} I_{22} I_{23}\right) I_{123}^{2} .
\end{aligned}
$$

Vector invariant $I_{123}$ is a common factor so by making $\Omega_{2}$ subject it can be written in terms of vector invariants of the adjoint action of $S E(3)$ acting on triple screws. Hence

$$
\begin{aligned}
\Omega_{2} & =\hat{I}_{123} I_{22}\left(I_{12} I_{23}-I_{13} I_{22}\right)+I_{123}\left(I_{12} \hat{I}_{22} I_{23}+I_{13} I_{22} \hat{I}_{22}\right. \\
& \left.+\hat{I}_{13} I_{22}^{2}-I_{22} I_{12} \hat{I}_{23}-I_{22} I_{23} \hat{I}_{12}\right) .
\end{aligned}
$$


Similar expression can be found for the offset numerators $\Omega_{1}, \Omega_{3}$.

Corollary 8.4.3. The offsets $d_{1}, d_{2}$, and $d_{3}$ can be expressed as vector invariants of the adjoint action SE(3) acting on triple screws as follows:

$$
\begin{aligned}
& d_{1}=\begin{array}{l}
\hat{I}_{123} I_{11}\left(I_{12} I_{13}-I_{11} I_{23}\right) \\
\quad+I_{123}\left(\hat{I}_{11}\left(I_{12} I_{13}+I_{11} I_{23}\right)+I_{11}\left(I_{11} \hat{I}_{23}-\hat{I}_{12} I_{13}-I_{12} \hat{I}_{13}\right)\right) \\
I_{11}^{\frac{1}{2}}\left(I_{11} I_{33}-I_{13}^{2}\right)\left(I_{11} I_{22}-I_{12}^{2}\right)
\end{array} \\
& d_{2}=\hat{I}_{123} I_{22}\left(I_{12} I_{23}-I_{13} I_{22}\right) \\
& \frac{+I_{123}\left(\hat{I}_{22}\left(I_{12} I_{23}+I_{13} I_{22}\right)+I_{22}\left(\hat{I}_{13} I_{22}-I_{12} \hat{I}_{23}-\hat{I}_{12} I_{23}\right)\right)}{I_{22}^{\frac{1}{2}}\left(I_{11} I_{22}-I_{12}^{2}\right)\left(I_{22} I_{33}-I_{23}^{2}\right)} \\
& d_{3}=\hat{I}_{123} I_{33}\left(I_{13} I_{23}-I_{12} I_{33}\right) \\
& \frac{+I_{123}\left(\hat{I}_{33}\left(I_{13} I_{23}+I_{12} I_{33}\right)+I_{33}\left(\hat{I}_{12} I_{33}-\hat{I}_{13} I_{23}-\hat{I}_{23} I_{13}\right)\right)}{I_{33}^{\frac{1}{2}}\left(I_{22} I_{33}-I_{23}^{2}\right)\left(I_{11} I_{33}-I_{13}^{2}\right)} .
\end{aligned}
$$

For the sake of completeness we give the formulae for a general $n$ - joint robot arm. Analogous to 8.10 we can write the link length $a_{i}$ between joints $i$ and $j=i+1$ is as follows:

$$
a_{i}=\frac{\boldsymbol{\omega}_{i} \cdot \boldsymbol{\omega}_{j}}{\left\|\boldsymbol{\omega}_{i} \times \boldsymbol{\omega}_{j}\right\|}\left(\frac{\boldsymbol{v}_{i} \cdot \boldsymbol{\omega}_{i}}{\boldsymbol{\omega}_{i} \cdot \boldsymbol{\omega}_{i}}+\frac{\boldsymbol{v}_{j} \cdot \boldsymbol{\omega}_{j}}{\boldsymbol{\omega}_{j} \cdot \boldsymbol{\omega}_{j}}\right)-\frac{\boldsymbol{\omega}_{i} \cdot \boldsymbol{v}_{j}+\boldsymbol{v}_{i} \cdot \boldsymbol{\omega}_{j}}{\left\|\boldsymbol{\omega}_{i} \times \boldsymbol{\omega}_{j}\right\|} .
$$

In the terms of vector invariants of the adjoint action of $S E(3)$

$$
a_{i}=\frac{I_{i j}}{\sqrt{I_{i i} I_{j j}-I_{i j}^{2}}}\left(\frac{\hat{I}_{i i}}{I_{i i}}+\frac{\hat{I}_{j j}}{I_{j j}}\right)-\frac{\hat{I}_{i j}}{I_{i i} I_{j j}-I_{i j}^{2}} .
$$

For the three successive joints $i, j=i+1$, and $k=i+2$ analogously to 8.25 the general formula of offset $d_{j}$ can be written as

$$
d_{j}=\left(\left|\boldsymbol{v}_{j} \boldsymbol{\omega}_{j} \boldsymbol{\omega}_{i}\right|\left(\boldsymbol{\omega}_{i} \cdot \boldsymbol{\omega}_{j}\right)+\left|\boldsymbol{v}_{i} \boldsymbol{\omega}_{i} \boldsymbol{\omega}_{j}\right|\left\|\boldsymbol{\omega}_{j}\right\|^{2}\right)\left\|\boldsymbol{\omega}_{k} \times \boldsymbol{\omega}_{j}\right\|^{2}
$$




$$
\frac{-\left(\left|\boldsymbol{v}_{j} \boldsymbol{\omega}_{j} \boldsymbol{\omega}_{k}\right|\left(\boldsymbol{\omega}_{k} \cdot \boldsymbol{\omega}_{j}\right)+\left|\boldsymbol{v}_{k} \boldsymbol{\omega}_{k} \boldsymbol{\omega}_{j}\right|\left\|\boldsymbol{\omega}_{j}\right\|^{2}\right)\left\|\boldsymbol{\omega}_{i} \times \boldsymbol{\omega}_{j}\right\|^{2}}{\left\|\boldsymbol{\omega}_{j}\right\|\left\|\boldsymbol{\omega}_{k} \times \boldsymbol{\omega}_{j}\right\|^{2}\left\|\boldsymbol{\omega}_{i} \times \boldsymbol{\omega}_{j}\right\|^{2}}
$$

We can write the numerator of offset $\Omega_{j}$ by using determinant of $4 \times 4$ matrix as

$$
\Omega_{j}=-\frac{1}{2}\left|\begin{array}{cccc}
\left|\boldsymbol{v}_{i} \boldsymbol{\omega}_{i} \boldsymbol{\omega}_{j}\right| & -\left(\boldsymbol{\omega}_{i} \cdot \boldsymbol{\omega}_{j}\right) & \left(\boldsymbol{\omega}_{i} \cdot \boldsymbol{\omega}_{j}\right) & \left(\boldsymbol{\omega}_{i} \cdot \boldsymbol{\omega}_{i}\right) \\
\left|\boldsymbol{v}_{j} \boldsymbol{\omega}_{j} \boldsymbol{\omega}_{i}\right| & \left(\boldsymbol{\omega}_{j} \cdot \boldsymbol{\omega}_{j}\right) & -\left(\boldsymbol{\omega}_{j} \cdot \boldsymbol{\omega}_{j}\right) & -\left(\boldsymbol{\omega}_{i} \cdot \boldsymbol{\omega}_{j}\right) \\
\left|\boldsymbol{v}_{j} \boldsymbol{\omega}_{j} \boldsymbol{\omega}_{k}\right| & -\left(\boldsymbol{\omega}_{j} \cdot \boldsymbol{\omega}_{j}\right) & -\left(\boldsymbol{\omega}_{j} \cdot \boldsymbol{\omega}_{j}\right) & -\left(\boldsymbol{\omega}_{j} \cdot \boldsymbol{\omega}_{k}\right) \\
\left|\boldsymbol{v}_{k} \boldsymbol{\omega}_{k} \boldsymbol{\omega}_{j}\right| & \left(\boldsymbol{\omega}_{j} \cdot \boldsymbol{\omega}_{k}\right) & \left(\boldsymbol{\omega}_{j} \cdot \boldsymbol{\omega}_{k}\right) & \left(\boldsymbol{\omega}_{k} \cdot \boldsymbol{\omega}_{k}\right)
\end{array}\right| .
$$

The offset $d_{j}$ can be written in terms of vector invariants of the adjoint action of $S E(3)$ acting of $n>3$ as follows:

$$
\begin{aligned}
& d_{j}=\hat{I}_{i j k} I_{j j}\left(I_{i j} I_{j k}-I_{i k} I_{j j}\right) \\
& \frac{+I_{i j k}\left(\hat{I}_{j j}\left(I_{i j} I_{j k}+I_{i k} I_{j j}\right)+I_{j j}\left(\hat{I}_{i k} I_{j j}-I_{i j} \hat{I}_{j k}-\hat{I}_{i j} I_{j k}\right)\right)}{I_{j j}^{\frac{1}{2}}\left(I_{i i} I_{j j}-I_{i j}^{2}\right)\left(I_{j j} I_{k k}-I_{j k}^{2}\right)} .
\end{aligned}
$$

Hence link lengths and offsets can be expressed in terms of vector invariants of the adjoint action of $S E(3)$ acting on $n$ screws using Plücker coordinates $\left(\boldsymbol{\omega}_{i}, \boldsymbol{v}_{i}\right)$. Moreover, they are examples of rational algebraic vector invariants for the same group under the same action. 


\section{Chapter 9}

\section{Geometric Duality for the Set of Three Screws}

In this chapter we are going to study the geometric interpretation of the Lie bracket of two screws and the consequences of this for sets of three screws. That interpretation will give us a clear picture of the relation between offsets and link lengths in the Denavit-Hartenberg parameters of the robot arms.

\subsection{The geometric interpretation of the Lie bracket of two screws}

The Lie bracket between two screws $S_{1}, S_{2}$ has the following form:

$$
\left[S_{1}, S_{2}\right]=\left(\boldsymbol{\omega}_{1} \times \boldsymbol{\omega}_{2}, \boldsymbol{\omega}_{1} \times \boldsymbol{v}_{2}+\boldsymbol{v}_{1} \times \boldsymbol{\omega}_{2}\right)
$$

where $S_{i}=\left(\boldsymbol{\omega}_{i}, \boldsymbol{v}_{i}\right)$ in Plücker coordinates. Samuel et al [45] give the physical meaning of the Lie bracket as a twist that acts on a screw whose axis is the common perpendicular to the axes of both screws. That screw has a pitch 
and we shall label it by $h_{3}^{\prime}$ which is equal

$$
h_{3}^{\prime}=h_{12}=\left(h_{1}+h_{2}\right)+a \cot \theta,
$$

where $h_{i}$ is the pitch of the screw $S_{i}$, the angle $\theta$ lies between the axes of $S_{1}$ and $S_{2}$, and $a$ is the distance between the axes of $S_{1}$ and $S_{2}$, which in this thesis is called the link length. The amplitude of a screw $S=(\boldsymbol{\omega}, \boldsymbol{v})$ is defined to be $\|\boldsymbol{\omega}\|$. Then the amplitude of $\left[S_{1}, S_{2}\right]$ is

$$
\left\|\boldsymbol{\omega}_{1} \times \boldsymbol{\omega}_{2}\right\|=\left\|\boldsymbol{\omega}_{1}\right\|\left\|\boldsymbol{\omega}_{2}\right\| \sin \theta
$$

\subsection{Axis of the common perpendicular}

Suppose we have a robot arm whose links are connected by three screws namely $S_{1}, S_{2}$, and $S_{3}$ with Plücker coordinates $\left(\boldsymbol{\omega}_{i}, \boldsymbol{v}_{i}\right)$ and $i=1,2,3$ respectively, and they are arbitrary in $\mathfrak{s e}(3)$. The Lie bracket between the screws $S_{1}$ and $S_{2}$ gives us a screw whose axis is the common perpendicular to $S_{1}$ and $S_{2}$ as above, and for convenience we label it by $S_{3}^{\prime}$ with Plücker coordinates

$$
\begin{aligned}
S_{3}^{\prime} & =\left(\boldsymbol{\omega}_{3}^{\prime}, \boldsymbol{v}_{3}^{\prime}\right)=\left[S_{1}, S_{2}\right] \\
& =\left(\boldsymbol{\omega}_{1} \times \boldsymbol{\omega}_{2}, \boldsymbol{\omega}_{1} \times \boldsymbol{v}_{2}+\boldsymbol{v}_{1} \times \boldsymbol{\omega}_{2}\right) .
\end{aligned}
$$

Similarly, the Lie bracket between the screws $S_{3}$ and $S_{1}$ gives us a screw whose axis is the common perpendicular to $S_{3}$ and $S_{1}$, and we label it by $S_{2}^{\prime}$ with Plücker coordinates

$$
S_{2}^{\prime}=\left(\boldsymbol{\omega}_{2}^{\prime}, \boldsymbol{v}_{2}^{\prime}\right)=\left[S_{3}, S_{1}\right]
$$




$$
=\left(\boldsymbol{\omega}_{3} \times \boldsymbol{\omega}_{1}, \boldsymbol{\omega}_{3} \times \boldsymbol{v}_{1}+\boldsymbol{v}_{3} \times \boldsymbol{\omega}_{1}\right),
$$

and Lie bracket between the screws $S_{2}$ and $S_{3}$ gives us a screw whose axis is the common perpendicular screw $S_{1}^{\prime}$ with Plücker coordinates

$$
\begin{aligned}
S_{1}^{\prime} & =\left(\boldsymbol{\omega}_{1}^{\prime}, \boldsymbol{v}_{1}^{\prime}\right)=\left[S_{2}, S_{3}\right] \\
& =\left(\boldsymbol{\omega}_{2} \times \boldsymbol{\omega}_{3}, \boldsymbol{\omega}_{2} \times \boldsymbol{v}_{3}+\boldsymbol{v}_{2} \times \boldsymbol{\omega}_{3}\right) .
\end{aligned}
$$

As a result of the way we labeled $S_{i}^{\prime}$ and the Lie bracket of any two of $S_{i}$ where $i=1,2,3$, we get these three equations which have a cyclic permutation of indices

$$
\begin{aligned}
& S_{1}^{\prime}=\left[S_{2}, S_{3}\right], \\
& S_{2}^{\prime}=\left[S_{3}, S_{1}\right], \\
& S_{3}^{\prime}=\left[S_{1}, S_{2}\right] .
\end{aligned}
$$

Definition 9.2.1. Given a set of three screws $S_{1}, S_{2}$, and $S_{3}$, their geometric dual is the set $S_{1}^{\prime}, S_{2}^{\prime}$, and $S_{3}^{\prime}$.

The set $S_{1}^{\prime}, S_{2}^{\prime}$, and $S_{3}^{\prime}$ creates a new set of screws related to the old one. Moreover, those two sets of screws enable us to find the relation between offsets and link lengths in Denavit-Hartenberg parameters of the robot as we shall see in the next sections.

\subsection{Invariants of geometric duals}

According to the relation between the new set of screws and the old one, the vector invariants of the adjoint action of $S E(3)$ acting on screws $S_{1}^{\prime}, S_{2}^{\prime}$, and 
$S_{3}^{\prime}$ are also related to the vector invariants of the adjoint action of $S E(3)$ acting on screws $S_{1}, S_{2}$, and $S_{3}$ stated in Theorem 6.3.1.

The vector invariants of the adjoint action of $S E(3)$ applied to the new screw triple $S_{1}^{\prime}, S_{2}^{\prime}$, and $S_{3}^{\prime}$ are denoted by a "dash", for instance $I_{11}^{\prime}$ for $I_{11}\left(S_{1}^{\prime}, S_{2}^{\prime}, S_{3}^{\prime}\right)$, when written in terms of Plücker coordinates of $\left(S_{1}, S_{2}, S_{3}\right)$ must still be invariant. This is because the adjoint action of Lie group is a Lie algebra automorphism, that is, if $g \in S E(3)$ and for any $S_{1}, S_{2} \in \mathfrak{s e}(3)$, then

$$
\left[g S_{1}, g S_{2}\right]=g\left[S_{1}, S_{2}\right]
$$

To calculate the vector invariants of the new set of screws and express them in terms of vector invariants of the old set of screws, we have to replace every Plücker coordinates of each old screw by the corresponding Plücker coordinates of the new screws, more precisely replacing $\boldsymbol{\omega}_{i}$ with $\boldsymbol{\omega}_{i}^{\prime}$, and $\boldsymbol{v}_{i}$ with $\boldsymbol{v}_{i}^{\prime}$ for $i=1,2,3$ in the vector invariants of the old set of screws and hence we get the vector invariants of the adjoint action of $S E(3)$ acting on the new screws.

For vector invariant $I_{11}=\boldsymbol{\omega}_{1} \cdot \boldsymbol{\omega}_{1}$, we have to replace $\boldsymbol{\omega}_{1}$ by $\boldsymbol{\omega}_{1}^{\prime}=\boldsymbol{\omega}_{2} \times \boldsymbol{\omega}_{3}$, and then using identity of Lagrange 8.8 as follows:

$$
\begin{aligned}
I_{11}^{\prime}=\boldsymbol{\omega}_{1}^{\prime} \cdot \boldsymbol{\omega}_{1}^{\prime} & =\left(\boldsymbol{\omega}_{2} \times \boldsymbol{\omega}_{3}\right) \cdot\left(\boldsymbol{\omega}_{2} \times \boldsymbol{\omega}_{3}\right) \\
& =\left(\boldsymbol{\omega}_{2} \cdot \boldsymbol{\omega}_{2}\right)\left(\boldsymbol{\omega}_{3} \cdot \boldsymbol{\omega}_{3}\right)-\left(\boldsymbol{\omega}_{2} \cdot \boldsymbol{\omega}_{3}\right)^{2} \\
& =I_{22} I_{33}-I_{23}^{2} .
\end{aligned}
$$

Analogously, replacing $\boldsymbol{\omega}_{2}^{\prime}$ by $\boldsymbol{\omega}_{3} \times \boldsymbol{\omega}_{1}$, and $\boldsymbol{\omega}_{3}^{\prime}$ by $\boldsymbol{\omega}_{1} \times \boldsymbol{\omega}_{2}$ we get

$$
I_{22}^{\prime}=\boldsymbol{\omega}_{2}^{\prime} \cdot \boldsymbol{\omega}_{2}^{\prime}=I_{11} I_{33}-I_{13}^{2} .
$$




$$
I_{33}^{\prime}=\boldsymbol{\omega}_{3}^{\prime} \cdot \boldsymbol{\omega}_{3}^{\prime}=I_{11} I_{22}-I_{12}^{2}
$$

For vector invariant $I_{12}=\boldsymbol{\omega}_{1} \cdot \boldsymbol{\omega}_{2}$, we have to replace $\boldsymbol{\omega}_{1}$ by $\boldsymbol{\omega}_{1}^{\prime}=\boldsymbol{\omega}_{2} \times \boldsymbol{\omega}_{3}$ and $\boldsymbol{\omega}_{2}$ by $\boldsymbol{\omega}_{2}^{\prime}=\boldsymbol{\omega}_{3} \times \boldsymbol{\omega}_{1}$ as follows:

$$
\begin{aligned}
I_{12}^{\prime}=\boldsymbol{\omega}_{1}^{\prime} \cdot \boldsymbol{\omega}_{2}^{\prime} & =\left(\boldsymbol{\omega}_{2} \times \boldsymbol{\omega}_{3}\right) \cdot\left(\boldsymbol{\omega}_{3} \times \boldsymbol{\omega}_{1}\right) \\
& =\left(\boldsymbol{\omega}_{3} \cdot \boldsymbol{\omega}_{1}\right)\left(\boldsymbol{\omega}_{2} \cdot \boldsymbol{\omega}_{3}\right)-\left(\boldsymbol{\omega}_{2} \cdot \boldsymbol{\omega}_{1}\right)\left(\boldsymbol{\omega}_{3} \cdot \boldsymbol{\omega}_{3}\right) \\
& =I_{13} I_{23}-I_{12} I_{33}
\end{aligned}
$$

Similarly, we get

$$
\begin{aligned}
& I_{13}^{\prime}=I_{23} I_{12}-I_{13} I_{22} . \\
& I_{23}^{\prime}=I_{12} I_{13}-I_{23} I_{11} .
\end{aligned}
$$

For vector invariant $\hat{I}_{11}=\boldsymbol{\omega}_{1} \cdot \boldsymbol{v}_{1}$, we have to replace $\boldsymbol{\omega}_{1}$ by $\boldsymbol{\omega}_{1}^{\prime}=\boldsymbol{\omega}_{2} \times \boldsymbol{\omega}_{3}$ and $\boldsymbol{v}_{1}$ by $\boldsymbol{v}_{1}^{\prime}=\boldsymbol{\omega}_{2} \times \boldsymbol{v}_{3}+\boldsymbol{v}_{2} \times \boldsymbol{\omega}_{3}$ as follows:

$$
\begin{aligned}
\hat{I}_{11}^{\prime}=\boldsymbol{\omega}_{1}^{\prime} \cdot \boldsymbol{v}_{1}^{\prime} & =\left(\boldsymbol{\omega}_{2} \times \boldsymbol{\omega}_{3}\right) \cdot\left(\boldsymbol{\omega}_{2} \times \boldsymbol{v}_{3}+\boldsymbol{v}_{2} \times \boldsymbol{\omega}_{3}\right), \\
& =\left(\boldsymbol{\omega}_{2} \times \boldsymbol{\omega}_{3}\right) \cdot\left(\boldsymbol{\omega}_{2} \times \boldsymbol{v}_{3}\right)+\left(\boldsymbol{\omega}_{2} \times \boldsymbol{\omega}_{3}\right) \cdot\left(\boldsymbol{v}_{2} \times \boldsymbol{\omega}_{3}\right), \\
& =\left(\boldsymbol{\omega}_{2} \cdot \boldsymbol{\omega}_{2}\right)\left(\boldsymbol{\omega}_{3} \cdot \boldsymbol{v}_{3}\right)-\left(\boldsymbol{\omega}_{2} \cdot \boldsymbol{v}_{3}\right)\left(\boldsymbol{\omega}_{2} \cdot \boldsymbol{\omega}_{3}\right) \\
& +\left(\boldsymbol{\omega}_{2} \cdot \boldsymbol{v}_{2}\right)\left(\boldsymbol{\omega}_{3} \cdot \boldsymbol{\omega}_{3}\right)-\left(\boldsymbol{\omega}_{3} \cdot \boldsymbol{v}_{3}\right)\left(\boldsymbol{\omega}_{2} \cdot \boldsymbol{\omega}_{3}\right) \\
& =\left(\boldsymbol{\omega}_{2} \cdot \boldsymbol{\omega}_{2}\right)\left(\boldsymbol{\omega}_{3} \cdot \boldsymbol{v}_{3}\right)+\left(\boldsymbol{\omega}_{2} \cdot \boldsymbol{v}_{2}\right)\left(\boldsymbol{\omega}_{3} \cdot \boldsymbol{\omega}_{3}\right) \\
& -\left(\boldsymbol{\omega}_{2} \cdot \boldsymbol{\omega}_{3}\right)\left(\boldsymbol{\omega}_{2} \cdot \boldsymbol{v}_{3}+\boldsymbol{\omega}_{3} \cdot \boldsymbol{v}_{3}\right), \\
& =I_{22} \hat{I}_{33}+\hat{I}_{22} I_{33}-\hat{I}_{23} I_{23} .
\end{aligned}
$$


Similarly, we get

$$
\begin{aligned}
& \hat{I}_{22}^{\prime}=I_{33} \hat{I}_{11}+\hat{I}_{33} I_{11}-\hat{I}_{13} I_{13} . \\
& \hat{I}_{33}^{\prime}=I_{11} \hat{I}_{22}+\hat{I}_{11} I_{22}-\hat{I}_{12} I_{12} . \\
& \hat{I}_{12}^{\prime}=\hat{I}_{13} I_{23}+\hat{I}_{23} I_{13}-\hat{I}_{12} I_{33}-2 \hat{I}_{33} I_{12} . \\
& \hat{I}_{13}^{\prime}=\hat{I}_{12} I_{23}+\hat{I}_{23} I_{12}-\hat{I}_{13} I_{22}-2 \hat{I}_{22} I_{13} . \\
& \hat{I}_{23}^{\prime}=\hat{I}_{12} I_{13}+\hat{I}_{13} I_{12}-\hat{I}_{23} I_{11}-2 \hat{I}_{11} I_{23} .
\end{aligned}
$$

For $I_{123}^{\prime}$ we substitute $\boldsymbol{\omega}_{1}^{\prime}=\boldsymbol{\omega}_{2} \times \boldsymbol{\omega}_{3}, \boldsymbol{\omega}_{2}^{\prime}=\boldsymbol{\omega}_{3} \times \boldsymbol{\omega}_{1}$, and $\boldsymbol{\omega}_{3}^{\prime}=\boldsymbol{\omega}_{1} \times \boldsymbol{\omega}_{2}$ in $\boldsymbol{\omega}_{1}^{\prime} \cdot\left(\boldsymbol{\omega}_{2}^{\prime} \times \boldsymbol{\omega}_{3}^{\prime}\right)$, and by using the vector triple product $\mathbf{a} \times(\mathbf{b} \times \mathbf{c})=$ $\mathbf{b}(\mathbf{a} \cdot \mathbf{c})-\mathbf{c}(\mathbf{a} \cdot \mathbf{b})[32]$, then we get

$$
\begin{aligned}
I_{123}^{\prime} & =\left(\boldsymbol{\omega}_{2} \times \boldsymbol{\omega}_{3}\right) \cdot\left(\left(\boldsymbol{\omega}_{3} \times \boldsymbol{\omega}_{1}\right) \times\left(\boldsymbol{\omega}_{1} \times \boldsymbol{\omega}_{2}\right)\right), \\
& =\left(\boldsymbol{\omega}_{2} \times \boldsymbol{\omega}_{3}\right) \cdot\left(\boldsymbol{\omega}_{3} \cdot\left(\boldsymbol{\omega}_{1} \times \boldsymbol{\omega}_{2}\right) \boldsymbol{\omega}_{1}\right), \\
& =\left(\boldsymbol{\omega}_{1} \cdot\left(\boldsymbol{\omega}_{2} \times \boldsymbol{\omega}_{3}\right)\right)^{2}, \\
& =I_{123}^{2} .
\end{aligned}
$$

The same argument applies for $\hat{I}_{123}^{\prime}$ and hence

$$
\hat{I}_{123}^{\prime}=2 I_{123} \hat{I}_{123}
$$

\subsection{Geometrical duality of offset and link length}

The physical interpretation of the Lie bracket of two screws in Section 9.1 tells us the position of the new set of screws relative to the old one and the 
relation between them. In particular, link lengths in the old set of screws become offsets in the new set set of screws, and offsets in the old set of screws become link lengths in the new one. That means there is a geometric duality between two sets of screws, for instance, link length $a_{1}$ after replacing $\boldsymbol{\omega}_{i}$ and $\boldsymbol{v}_{i}$ by $\boldsymbol{\omega}_{i}^{\prime}$ and $\boldsymbol{v}_{i}^{\prime}$ becomes $a_{1}^{\prime}$ and equal the offset $d_{3}$. Similarly $a_{2}$ becomes $a_{2}^{\prime}=d_{1}$, and $a_{3}$ becomes $a_{3}^{\prime}=d_{2}$. The converse is also true, for instance, offset $d_{1}$ after replacing $\boldsymbol{\omega}_{i}$ and $\boldsymbol{v}_{i}$ by $\boldsymbol{\omega}_{i}^{\prime}$ and $\boldsymbol{v}_{i}^{\prime}$ becomes $d_{1}^{\prime}$ and equal to link length $a_{2}$, and so on for the others.

Theorem 9.4.1. Given a set of three screws, the Denavit-Hartenberg parameters of its geometric dual are as follows

$$
\begin{array}{lll}
d_{1}^{\prime}=a_{2}, & d_{2}^{\prime}=a_{3}, & d_{3}^{\prime}=a_{1}, \\
a_{1}^{\prime}=d_{3}, & a_{2}^{\prime}=d_{1}, & a_{3}^{\prime}=d_{2} .
\end{array}
$$

Proof. If we replace every $\boldsymbol{\omega}_{i}$ and $\boldsymbol{v}_{i}$ of offset $d_{i}$ in the original set of screws by $\boldsymbol{\omega}_{i}^{\prime}$ and $\boldsymbol{v}_{i}^{\prime}$ then it becomes $d_{i}^{\prime}$ which is equal to the link length in the associated set of screws. To avoid long tedious calculations we have done the calculations by using the Maple program, see Section A.2.

\subsection{Geometrically dual set of screws}

In this section we justify the term geometric duality by the corresponding Lie brackets of the screws $S_{1}^{\prime}, S_{2}^{\prime}$, and $S_{3}^{\prime}$. Bear in mind that the Lie brackets physically give us the screw whose axis is the common perpendicular to the axes of the original screws and we denote them as

$$
S_{1}^{\prime \prime}=\left[S_{2}^{\prime}, S_{3}^{\prime}\right]
$$




$$
\begin{aligned}
& S_{2}^{\prime \prime}=\left[S_{3}^{\prime}, S_{1}^{\prime}\right] . \\
& S_{3}^{\prime \prime}=\left[S_{1}^{\prime}, S_{2}^{\prime}\right] .
\end{aligned}
$$

In order to know the relation between $S_{i}^{\prime \prime}$ and $S_{i}$ we have to expand these Lie brackets. Its enough to calculate one of them and get a generalisation.

$$
\begin{aligned}
S_{1}^{\prime \prime} & =\left[S_{2}^{\prime}, S_{3}^{\prime}\right] \\
& =\left[\left(\boldsymbol{\omega}_{3} \times \boldsymbol{\omega}_{1}, \boldsymbol{\omega}_{3} \times \boldsymbol{v}_{1}+\boldsymbol{v}_{3} \times \boldsymbol{\omega}_{1}\right),\left(\boldsymbol{\omega}_{1} \times \boldsymbol{\omega}_{2}, \boldsymbol{\omega}_{1} \times \boldsymbol{v}_{2}+\boldsymbol{v}_{1} \times \boldsymbol{\omega}_{2}\right)\right] \\
& =\left(\boldsymbol{\omega}_{3} \times \boldsymbol{\omega}_{1} \times \boldsymbol{\omega}_{1} \times \boldsymbol{\omega}_{2},\left(\boldsymbol{\omega}_{3} \times \boldsymbol{\omega}_{1}\right) \times\left(\boldsymbol{\omega}_{1} \times \boldsymbol{v}_{2}+\boldsymbol{v}_{1} \times \boldsymbol{\omega}_{2}\right)\right. \\
& \left.+\left(\boldsymbol{\omega}_{3} \times \boldsymbol{v}_{1}+\boldsymbol{v}_{3} \times \boldsymbol{\omega}_{1}\right) \times\left(\boldsymbol{\omega}_{1} \times \boldsymbol{\omega}_{2}\right)\right) \\
& =\left(\boldsymbol{\omega}_{1}\left(\left(\boldsymbol{\omega}_{3} \times \boldsymbol{\omega}_{1}\right) \cdot \boldsymbol{\omega}_{2}\right), \boldsymbol{\omega}_{1}\left(\boldsymbol{v}_{1} \cdot\left(\boldsymbol{\omega}_{2} \times \boldsymbol{\omega}_{3}\right)+\boldsymbol{v}_{2} \cdot\left(\boldsymbol{\omega}_{3} \times \boldsymbol{\omega}_{1}\right)+\boldsymbol{v}_{3} \cdot\left(\boldsymbol{\omega}_{1} \times \boldsymbol{\omega}_{2}\right)\right)\right. \\
& \left.+\boldsymbol{v}_{1}\left(\left(\boldsymbol{\omega}_{3} \times \boldsymbol{\omega}_{1}\right) \cdot \boldsymbol{\omega}_{2}\right)\right) .
\end{aligned}
$$

Note that the coefficients are in fact invariants hence

$$
\begin{aligned}
& =\left(I_{123} \boldsymbol{\omega}_{1}, \hat{I}_{123} \boldsymbol{\omega}_{1}+I_{123} \boldsymbol{v}_{1}\right) \\
& =\left(I_{123}+\epsilon \hat{I}_{123}\right)\left(\boldsymbol{\omega}_{1}+\epsilon \boldsymbol{v}_{1}\right) \\
& =\left(I_{123}+\epsilon \hat{I}_{123}\right) S_{1} .
\end{aligned}
$$

Given that $I_{123}$ represents the volume of a parallelepiped spanned by $\boldsymbol{\omega}_{1}, \boldsymbol{\omega}_{2}$, and $\boldsymbol{\omega}_{3}$. We can think of this scaling factor as a dual volume.

Observe that $S_{1}^{\prime \prime}, S_{2}^{\prime \prime}, S_{3}^{\prime \prime}$ are the same as $S_{1}, S_{2}, S_{3}$ except for the factor $\left(I_{123}+\epsilon \hat{I}_{123}\right)$, and hence

$$
\left(S_{1}^{\prime \prime}, S_{2}^{\prime \prime}, S_{3}^{\prime \prime}\right)=\left(I_{123}+\epsilon \hat{I}_{123}\right)\left(S_{1}, S_{2}, S_{3}\right)
$$


Geometrically, the screws $S_{i}^{\prime \prime}$ and $S_{i}$ have the same axes but their pitches differ as a result of that factor. We denote the pitch of $S_{i}$ by $h_{i}$ as we mentioned before whereas we will denote the pitch of $S_{i}^{\prime \prime}$ by $h_{i}^{\prime \prime}$. Then for $i=1,2,3$ :

$$
\begin{aligned}
h_{i}^{\prime \prime} & =\frac{I_{123} \boldsymbol{\omega}_{i} \cdot\left(\hat{I}_{123} \boldsymbol{\omega}_{i}+I_{123} \boldsymbol{v}_{i}\right)}{I_{123} \boldsymbol{\omega}_{i} \cdot I_{123} \boldsymbol{\omega}_{i}} \\
& =\frac{I_{123} \hat{I}_{123} \boldsymbol{\omega}_{i} \cdot \boldsymbol{\omega}_{i}+I_{123}^{2} \boldsymbol{\omega}_{i} \cdot \boldsymbol{v}_{i}}{I_{123}^{2} \boldsymbol{\omega}_{i} \cdot \boldsymbol{\omega}_{i}} \\
& =\frac{\hat{I}_{123}}{I_{123}}+\frac{\boldsymbol{\omega}_{i} \cdot \boldsymbol{v}_{i}}{\boldsymbol{\omega}_{i} \cdot \boldsymbol{\omega}_{i}} \\
& =\frac{\hat{I}_{123}}{I_{123}}+h_{i} .
\end{aligned}
$$




\section{Appendix A}

\section{Maple Output}

\section{A.1 Rank of algebraically independent vec- tor invariants}

Maple helps us to find the rank of any $n \times m$ matrix. Jacobian matrix $J$ consists of 18 rows and 12 columns, where rows represent the partial derivatives with respect to 18 variables $w_{11}, \cdots, v_{33}$ and columns represent the first 12 vector invariants of the adjoint action of $S E(3)$ acting on triple screws stated in Theorem 6.3.1. According to Theorem 6.3.2, if the rank of $J$ equal 12 , then those 12 vector invariants are algebraically independent. 
with (LinearAlgebra) : interface $($ rtablesize $=20)$;

$d:=\operatorname{Matrix}\left(\left[\left[2 w_{11}, 0,0, w_{21}, w_{31}, 0, v_{11}, 0,0, v_{21}, v_{31}, 0\right],\left[2 w_{12}, 0,0, w_{22}, w_{32}, 0, v_{12}, 0,0, v_{22}, v_{32}\right.\right.\right.$, $0],\left[2 w_{13}, 0,0, w_{23}, w_{33}, 0, v_{13}, 0,0, v_{23}, v_{33}, 0\right],\left[0,2 w_{21}, 0, w_{11}, 0, w_{31}, 0, v_{21}, 0, v_{11}, 0, v_{31}\right],[0$, $\left.2 w_{22}, 0, w_{12}, 0, w_{32}, 0, v_{22}, 0, v_{12}, 0, v_{32}\right],\left[0,2 w_{23}, 0, w_{13}, 0, w_{33}, 0, v_{23}, 0, v_{13}, 0, v_{33}\right],[0,0$, $\left.2 w_{31}, 0, w_{11}, w_{21}, 0,0, v_{31}, 0, v_{11}, v_{21}\right],\left[0,0,2 w_{32}, 0, w_{12}, w_{22}, 0,0, v_{32}, 0, v_{12}, v_{22}\right],\left[0,0,2 w_{33}\right.$, $\left.0, w_{13}, w_{23}, 0,0, v_{33}, 0, v_{13}, v_{23}\right],\left[0,0,0,0,0,0, w_{11}, 0,0, w_{21}, w_{31}, 0\right],\left[0,0,0,0,0,0, w_{12}, 0,0\right.$, $\left.w_{22}, w_{32}, 0\right],\left[0,0,0,0,0,0, w_{13}, 0,0, w_{23}, w_{33}, 0\right],\left[0,0,0,0,0,0,0, w_{21}, 0, w_{11}, 0, w_{31}\right],[0,0,0$, $\left.0,0,0,0, w_{22}, 0, w_{12}, 0, w_{32}\right],\left[0,0,0,0,0,0,0, w_{23}, 0, w_{13}, 0, w_{33}\right],\left[0,0,0,0,0,0,0,0, w_{31}, 0\right.$ $\left.\left.\left.w_{11}, w_{21}\right],\left[0,0,0,0,0,0,0,0, w_{32}, 0, w_{12}, w_{22}\right],\left[0,0,0,0,0,0,0,0, w_{33}, 0, w_{13}, w_{23}\right]\right]\right)$;

$$
\left[\begin{array}{cccccccccccc}
2 w_{11} & 0 & 0 & w_{21} & w_{31} & 0 & v_{11} & 0 & 0 & v_{21} & v_{31} & 0 \\
2 w_{12} & 0 & 0 & w_{22} & w_{32} & 0 & v_{12} & 0 & 0 & v_{22} & v_{32} & 0 \\
2 w_{13} & 0 & 0 & w_{23} & w_{33} & 0 & v_{13} & 0 & 0 & v_{23} & v_{33} & 0 \\
0 & 2 w_{21} & 0 & w_{11} & 0 & w_{31} & 0 & v_{21} & 0 & v_{11} & 0 & v_{31} \\
0 & 2 w_{22} & 0 & w_{12} & 0 & w_{32} & 0 & v_{22} & 0 & v_{12} & 0 & v_{32} \\
0 & 2 w_{23} & 0 & w_{13} & 0 & w_{33} & 0 & v_{23} & 0 & v_{13} & 0 & v_{33} \\
0 & 0 & 2 w_{31} & 0 & w_{11} & w_{21} & 0 & 0 & v_{31} & 0 & v_{11} & v_{21} \\
0 & 0 & 2 w_{32} & 0 & w_{12} & w_{22} & 0 & 0 & v_{32} & 0 & v_{12} & v_{22} \\
0 & 0 & 2 w_{33} & 0 & w_{13} & w_{23} & 0 & 0 & v_{33} & 0 & v_{13} & v_{23} \\
0 & 0 & 0 & 0 & 0 & 0 & w_{11} & 0 & 0 & w_{21} & w_{31} & 0 \\
0 & 0 & 0 & 0 & 0 & 0 & w_{12} & 0 & 0 & w_{22} & w_{32} & 0 \\
0 & 0 & 0 & 0 & 0 & 0 & w_{13} & 0 & 0 & w_{23} & w_{33} & 0 \\
0 & 0 & 0 & 0 & 0 & 0 & 0 & w_{21} & 0 & w_{11} & 0 & w_{31} \\
0 & 0 & 0 & 0 & 0 & 0 & 0 & w_{22} & 0 & w_{12} & 0 & w_{32} \\
0 & 0 & 0 & 0 & 0 & 0 & 0 & w_{23} & 0 & w_{13} & 0 & w_{33} \\
0 & 0 & 0 & 0 & 0 & 0 & 0 & 0 & w_{31} & 0 & w_{11} & w_{21} \\
0 & 0 & 0 & 0 & 0 & 0 & 0 & w_{32} & 0 & w_{12} & w_{22} \\
0 & 0 & 0 & 0 & 0 & 0 & 0 & w_{33} & 0 & w_{13} & w_{23}
\end{array}\right]
$$

$\operatorname{Rank}(d)$ 


\section{A.2 Geometric duality between link lengths and offsets}

Recall that we denote the vector invariants of the adjoint action of $S E(3)$ acting on triple screws $S_{1}, S_{2}$, and $S_{3}$ by $I_{i j}$ for those vector invariants that have $\boldsymbol{\omega}_{i} \cdot \boldsymbol{\omega}_{j}$ where $i, j=1,2,3$, and for those vector invariants that have $v$ 's we denote them by $\hat{I}_{i j}$. However, for this program we can not write $\hat{I}_{i j}$ in Maple, so we will denote $\hat{I}_{i j}$ by $H_{i j}$. Furthermore, in the new system of screws $S_{1}^{\prime}, S_{2}^{\prime}$, and $S_{3}^{\prime}$, we denote $I_{i j}^{\prime}$ by $p i j$, for instance, $p 12$ correspond to $I_{12}^{\prime}$, and pijh correspond to $\hat{I}_{i j}^{\prime}$. Moreover, $p 123$ represents $I_{123}^{2}$ and $p 123 h$ represents $2 I_{123} \hat{I}_{123}$. The first syzygy $I_{123}^{2}$ is denoted by $a$ where as the second syzygy is denoted by $j$.

We denote the numerator and dominator of offset $d_{1}$ by $\Omega_{1}$ and dom1. We denote the other offsets similarly. We still denote link length number one by $a_{1}$.

In this program, we have only done the geometric duality from offsets to link length. However, the converse can also be shown to be true by tedious straight forward calculations. 


$$
\begin{aligned}
& p 11:=I_{22} \cdot I_{33}-P_{23}^{2} \\
& p 22:=I_{11} \cdot I_{33}-P_{13}^{2} \\
& p 33:=I_{11} \cdot I_{22}-P_{12} \\
& p 12:=I_{23} \cdot I_{13}-I_{12} \cdot I_{33} \\
& p 13:=I_{12} \cdot I_{23}-I_{22} \cdot I_{13} \\
& p 23:=I_{13} \cdot I_{12}-I_{11} \cdot I_{23} \\
& p 11 h:=I_{22} \cdot H_{33}+H_{22} \cdot I_{33}-H_{23} \cdot I_{23} \\
& p 22 h:=I_{33} \cdot H_{11}+H_{33} \cdot I_{11}-H_{13} \cdot I_{13} \\
& \mathrm{I}_{22} H_{33}+H_{22} \mathrm{I}_{33}-H_{23} \mathrm{I}_{23} \\
& \mathrm{I}_{33} H_{11}+H_{33} \mathrm{I}_{11}-H_{13} \mathrm{I}_{13} \\
& p 33 h:=I_{11} \cdot H_{22}+H_{11} \cdot I_{22}-H_{12} \cdot I_{12} \\
& \mathrm{I}_{11} H_{22}+H_{11} \mathrm{I}_{22}-H_{12} \mathrm{I}_{12} \\
& p 12 h:=-2 \cdot H_{33} \cdot I_{12}+H_{13} \cdot I_{23}-H_{12} \cdot I_{33}+H_{23} \cdot I_{13} \\
& -2 H_{33} \mathrm{I}_{12}+H_{13} \mathrm{I}_{23}-H_{12} \mathrm{I}_{33}+H_{23} \mathrm{I}_{13} \\
& p 13 h:=-2 \cdot H_{22} \cdot I_{13}-H_{13} \cdot I_{22}+H_{12} \cdot I_{23}+H_{23} \cdot I_{12} \\
& -2 \mathrm{H}_{22} \mathrm{I}_{13}-\mathrm{H}_{13} \mathrm{I}_{22}+H_{12} \mathrm{I}_{23}+H_{23} \mathrm{I}_{12} \\
& p 23 h:=-2 \cdot H_{11} \cdot I_{23}-H_{23} \cdot I_{11}+H_{12} \cdot I_{13}+H_{13} \cdot I_{12} \\
& -2 H_{11} \mathrm{I}_{23}-H_{23} \mathrm{I}_{11}+H_{12} \mathrm{I}_{13}+H_{13} \mathrm{I}_{12} \\
& \text { p123 }:=I_{11} \cdot I_{22} \cdot I_{33}-I_{11} \cdot P_{23}-P_{12}^{2} \cdot I_{33}+2 \cdot I_{12} \cdot I_{13} \cdot I_{23}-P_{13} \cdot I_{22} \\
& \mathrm{I}_{11} \mathrm{I}_{22} \mathrm{I}_{33}-\mathrm{I}_{11} \mathrm{I}_{23}^{2}-\mathrm{I}_{12}^{2} \mathrm{I}_{33}+2 \mathrm{I}_{12} \mathrm{I}_{13} \mathrm{I}_{23}-\mathrm{I}_{13}^{2} \mathrm{I}_{22} \\
& 2 \mathrm{I}_{123} H_{123} \\
& \mathrm{I}_{11} \mathrm{I}_{22} \mathrm{I}_{33}-\mathrm{I}_{11} \mathrm{I}_{23}-\mathrm{I}_{12}^{2} \mathrm{I}_{33}+2 \mathrm{I}_{12} \mathrm{I}_{13} \mathrm{I}_{23}-\mathrm{I}_{13}^{2} \mathrm{I}_{22}
\end{aligned}
$$




$$
\begin{aligned}
& \operatorname{dom} 1:=p 11 \cdot \operatorname{simplify}\left(\left(p 22 \cdot p 11-p 12^{2}\right) \cdot\left(p 33 \cdot p 11-p 13^{2}\right)\right) \\
& \left(\mathrm{I}_{22} \mathrm{I}_{33}-\mathrm{I}_{23}^{2}\right) \mathrm{I}_{33}\left(\mathrm{I}_{11} \mathrm{I}_{22} \mathrm{I}_{33}-\mathrm{I}_{11} \mathrm{I}_{23}^{2}-\mathrm{I}_{12}^{2} \mathrm{I}_{33}+2 \mathrm{I}_{12} \mathrm{I}_{13} \mathrm{I}_{23}-\mathrm{I}_{13}^{2} \mathrm{I}_{22}\right)^{2} \mathrm{I}_{22} \\
& a_{2}:=\operatorname{simplify}\left(\frac{\Omega_{1}}{\operatorname{dom} 1}\right) \\
& -\frac{1 .\left(\mathrm{I}_{22} \mathrm{H}_{23} \mathrm{I}_{33}-1 . \mathrm{I}_{22} \mathrm{H}_{33} \mathrm{I}_{23}-1 . H_{22} \mathrm{I}_{23} \mathrm{I}_{33}\right)}{\mathrm{I}_{22} \mathrm{I}_{33}\left(\mathrm{I}_{22} \mathrm{I}_{33}-1 . \mathrm{I}_{23}^{2}\right)}
\end{aligned}
$$

$\Omega_{2}:=\operatorname{simplify}\left(2 . j \cdot p 22 \cdot(p 12 \cdot p 23-p 13 \cdot p 22)+p 123 \cdot\left(p 22 h \cdot(p 12 \cdot p 23+p 13 \cdot p 22)+p 13 h \cdot p 22^{2}-p 23 h \cdot p 22 \cdot p 12-p 12 h \cdot p 22 \cdot p 23\right)\right)$

4. $H_{11} \mathrm{I}_{22} \mathrm{I}_{33}^{2} \mathrm{I}_{11} \mathrm{I}_{23} \mathrm{I}_{13}^{2} \mathrm{I}_{12}+4 . H_{33} \mathrm{I}_{11}^{2} \mathrm{I}_{22} \mathrm{I}_{33} \mathrm{I}_{23} \mathrm{I}_{13}^{2} \mathrm{I}_{12}+4 . H_{13} \mathrm{I}_{13}^{3} \mathrm{I}_{22} \mathrm{I}_{11} \mathrm{I}_{33} \mathrm{I}_{23} \mathrm{I}_{12}-4 . H_{13} \mathrm{I}_{12} \mathrm{I}_{23} \mathrm{I}_{11} \mathrm{I}_{33}^{2} \mathrm{I}_{22} \mathrm{I}_{13}+2 . \mathrm{I}_{11}^{2} \mathrm{I}_{22} \mathrm{I}_{33}^{3} H_{13} \mathrm{I}_{12}^{2}$

+2. $\mathrm{I}_{11}^{3} \mathrm{I}_{22} \mathrm{I}_{33}^{2} H_{13} \mathrm{I}_{23}^{2}-4 . \mathrm{I}_{11}^{2} \mathrm{I}_{23}^{3} H_{33} \mathrm{I}_{12} \mathrm{I}_{13}^{2}-2 . \mathrm{I}_{11}^{2} \mathrm{I}_{23}^{2} H_{13} \mathrm{I}_{12}^{2} \mathrm{I}_{33}^{2}-4 . \mathrm{I}_{12}^{3} \mathrm{I}_{33}^{2} H_{11} \mathrm{I}_{23} \mathrm{I}_{13}^{2}+H_{11} \mathrm{I}_{23}^{4} \mathrm{I}_{11}^{2} \mathrm{I}_{33} \mathrm{I}_{13}+4 . H_{11} \mathrm{I}_{23}^{2} \mathrm{I}_{13}^{3}$

$\mathrm{I}_{12}^{2} \mathrm{I}_{33}+H_{33} \mathrm{I}_{11}^{3} \mathrm{I}_{22}^{2} \mathrm{I}_{33}^{2} \mathrm{I}_{13}-2 . H_{33} \mathrm{I}_{11}^{2} \mathrm{I}_{22}^{2} \mathrm{I}_{33} \mathrm{I}_{13}^{3}+2 . H_{33} \mathrm{I}_{11}^{2} \mathrm{I}_{22} \mathrm{I}_{13}^{3} \mathrm{I}_{23}^{2}+H_{33} \mathrm{I}_{12}^{4} \mathrm{I}_{11} \mathrm{I}_{33}^{2} \mathrm{I}_{13}+4 . H_{33} \mathrm{I}_{12}^{2} \mathrm{I}_{13}^{3} \mathrm{I}_{23}^{2} \mathrm{I}_{11}+2 \cdot H_{13} \mathrm{I}_{13}^{2} \mathrm{I}_{22}^{2}$

$\mathrm{I}_{11}^{2} \mathrm{I}_{33}^{2}-1 . H_{13} \mathrm{I}_{13}^{4} \mathrm{I}_{22}^{2} \mathrm{I}_{11} \mathrm{I}_{33}+H_{11} \mathrm{I}_{22}^{2} \mathrm{I}_{33}^{3} \mathrm{I}_{11}^{2} \mathrm{I}_{13}-2 . H_{11} \mathrm{I}_{22}^{2} \mathrm{I}_{33}^{2} \mathrm{I}_{11} \mathrm{I}_{13}^{3}+2 . H_{11} \mathrm{I}_{22} \mathrm{I}_{33}^{2} \mathrm{I}_{13}^{3} \mathrm{I}_{12}^{2}-2 . H_{33} \mathrm{I}_{11}^{3} \mathrm{I}_{22} \mathrm{I}_{33} \mathrm{I}_{23}^{2} \mathrm{I}_{13}-2 . H_{33}$

$\mathrm{I}_{11}^{2} \mathrm{I}_{22} \mathrm{I}_{33}^{2} \mathrm{I}_{12}^{2} \mathrm{I}_{13}-4 . H_{33} \mathrm{I}_{11} \mathrm{I}_{22} \mathrm{I}_{13}^{4} \mathrm{I}_{23} \mathrm{I}_{12}+2 . H_{33} \mathrm{I}_{11} \mathrm{I}_{22} \mathrm{I}_{13}^{3} \mathrm{I}_{12}^{2} \mathrm{I}_{33}-4 . H_{33} \mathrm{I}_{12}^{3} \mathrm{I}_{11} \mathrm{I}_{33} \mathrm{I}_{23} \mathrm{I}_{13}^{2}+2 . H_{33} \mathrm{I}_{12} \mathrm{I}_{11}^{2} \mathrm{I}_{33} \mathrm{I}_{23}^{2} \mathrm{I}_{13}-2 . H_{13}$

$\mathrm{I}_{13}^{2} \mathrm{I}_{22} \mathrm{I}_{11}^{2} \mathrm{I}_{33} \mathrm{I}_{23}^{2}-2 . H_{13} \mathrm{I}_{13}^{2} \mathrm{I}_{22} \mathrm{I}_{11} \mathrm{I}_{33}^{2} \mathrm{I}_{12}^{2}-4 . H_{13} \mathrm{I}_{12}^{2} \mathrm{I}_{23}^{2} \mathrm{I}_{11} \mathrm{I}_{33} \mathrm{I}_{13}^{2}+4 . H_{13} \mathrm{I}_{12} \mathrm{I}_{23}^{3} \mathrm{I}_{11}^{2} \mathrm{I}_{33} \mathrm{I}_{13}+4 . H_{13} \mathrm{I}_{12}^{3} \mathrm{I}_{23} \mathrm{I}_{11} \mathrm{I}_{33}^{2} \mathrm{I}_{13}$

- 2. $H_{11} \mathrm{I}_{22} \mathrm{I}_{33}^{2} \mathrm{I}_{11}^{2} \mathrm{I}_{23}^{2} \mathrm{I}_{13}-2 . H_{11} \mathrm{I}_{22} \mathrm{I}_{33}^{3} \mathrm{I}_{11} \mathrm{I}_{12}^{2} \mathrm{I}_{13}-4 . H_{11} \mathrm{I}_{22} \mathrm{I}_{33} \mathrm{I}_{13}^{4} \mathrm{I}_{23} \mathrm{I}_{12}+2 . H_{11} \mathrm{I}_{22} \mathrm{I}_{33} \mathrm{I}_{13}^{3} \mathrm{I}_{23}^{2} \mathrm{I}_{11}-4 . H_{11} \mathrm{I}_{23}^{3} \mathrm{I}_{11} \mathrm{I}_{33} \mathrm{I}_{13}^{2} \mathrm{I}_{12}$

$+2 . H_{11} \mathrm{I}_{23}^{2} \mathrm{I}_{11} \mathrm{I}_{33}^{2} \mathrm{I}_{12}^{2} \mathrm{I}_{13}-1 . \mathrm{I}_{11}^{3} \mathrm{I}_{22}^{2} \mathrm{I}_{33}^{3} H_{13}+\mathrm{I}_{11}^{3} \mathrm{I}_{23}^{4} H_{33} \mathrm{I}_{13}-1 . \mathrm{I}_{11}^{3} \mathrm{I}_{23}^{4} H_{13} \mathrm{I}_{33}+\mathrm{I}_{12}^{4} \mathrm{I}_{33}^{3} H_{11} \mathrm{I}_{13}-1 . \mathrm{I}_{12}^{4} \mathrm{I}_{33}^{3} H_{13} \mathrm{I}_{11}+H_{11}$

$\mathrm{I}_{22}^{2} \mathrm{I}_{33} \mathrm{P}_{13}^{5}+\mathrm{H}_{33} \mathrm{I}_{11} \mathrm{I}_{22}^{2} \mathrm{P}_{13}^{5}$

$\operatorname{dom} 2:=p 22 \cdot \operatorname{simplify}\left(\left(p 22 \cdot p 33-p 23^{2}\right) \cdot\left(p 22 \cdot p 11-p 12^{2}\right)\right)$

$$
\left(\mathrm{I}_{11} \mathrm{I}_{33}-\mathrm{I}_{13}^{2}\right) \mathrm{I}_{11}\left(\mathrm{I}_{11} \mathrm{I}_{22} \mathrm{I}_{33}-\mathrm{I}_{11} \mathrm{I}_{23}^{2}-\mathrm{I}_{12}^{2} \mathrm{I}_{33}+2 \mathrm{I}_{12} \mathrm{I}_{13} \mathrm{I}_{23}-\mathrm{I}_{13}^{2} \mathrm{I}_{22}\right)^{2} \mathrm{I}_{33}
$$

$a_{3}:=\operatorname{simplify}\left(\frac{\Omega_{2}}{\operatorname{dom} 2}\right)$

$$
-\frac{1 .\left(\mathrm{I}_{11} H_{13} \mathrm{I}_{33}-1 . \mathrm{I}_{11} H_{33} \mathrm{I}_{13}-1 . H_{11} \mathrm{I}_{13} \mathrm{I}_{33}\right)}{\mathrm{I}_{33} \mathrm{I}_{11}\left(\mathrm{I}_{11} \mathrm{I}_{33}-1 . \mathrm{I}_{13}^{2}\right)}
$$

$\Omega_{3}:=\operatorname{simplify}\left(2 . j \cdot p 33 \cdot(p 13 \cdot p 23-p 12 \cdot p 33)+p 123 \cdot\left(p 33 h \cdot(p 13 \cdot p 23+p 12 \cdot p 33)+p 12 h \cdot p 33^{2}-p 13 h \cdot p 33 \cdot p 23-p 23 h \cdot p 33 \cdot p 13\right)\right)$ $H_{22} \mathrm{I}_{13}^{4} \mathrm{I}_{11} \mathrm{I}_{22}^{2} \mathrm{I}_{12}+4 . H_{22} \mathrm{I}_{13}^{2} \mathrm{I}_{12}^{3} \mathrm{I}_{23}^{2} \mathrm{I}_{11}+H_{22} \mathrm{I}_{11}^{3} \mathrm{I}_{33}^{2} \mathrm{I}_{22}^{2} \mathrm{I}_{12}-2 . H_{22} \mathrm{I}_{11}^{2} \mathrm{I}_{33}^{2} \mathrm{I}_{22} \mathrm{I}_{12}^{3}+2 . H_{22} \mathrm{I}_{11}^{2} \mathrm{I}_{33} \mathrm{I}_{12}^{3} \mathrm{I}_{23}^{2}+2 . H_{12} \mathrm{I}_{12}^{2} \mathrm{I}_{33}^{2} \mathrm{I}_{11} \mathrm{I}_{22}^{2}-1 . H_{12}$

$\mathrm{I}_{12}^{4} \mathrm{I}_{33}^{2} \mathrm{I}_{11} \mathrm{I}_{22}-4 . \mathrm{I}_{12}^{2} \mathrm{I}_{13}^{3} \mathrm{I}_{23} H_{11} \mathrm{I}_{22}^{2}-2 . \mathrm{I}_{11}^{2} \mathrm{I}_{23}^{2} H_{12} \mathrm{I}_{13}^{2} \mathrm{I}_{22}^{2}+2 . \mathrm{I}_{11}^{3} \mathrm{I}_{22}^{2} \mathrm{I}_{33} H_{12} \mathrm{I}_{23}^{2}+2 . \mathrm{I}_{11}^{2} \mathrm{I}_{22}^{3} \mathrm{I}_{33} H_{12} \mathrm{I}_{13}^{2}-4 . \mathrm{I}_{11}^{2} \mathrm{I}_{23}^{3} H_{22} \mathrm{I}_{13} \mathrm{I}_{12}^{2}$

+2. $H_{11} \mathrm{I}_{22}^{2} \mathrm{I}_{33} \mathrm{I}_{12}^{3} \mathrm{I}_{13}^{2}+H_{11} \mathrm{I}_{23}^{4} \mathrm{I}_{11}^{2} \mathrm{I}_{22} \mathrm{I}_{12}+4 . H_{11} \mathrm{I}_{23}^{2} \mathrm{I}_{12}^{3} \mathrm{I}_{22} \mathrm{I}_{13}^{2}+H_{11} \mathrm{I}_{22}^{3} \mathrm{I}_{33}^{2} \mathrm{I}_{11}^{2} \mathrm{I}_{12}-2 . H_{11} \mathrm{I}_{22}^{2} \mathrm{I}_{33}^{2} \mathrm{I}_{11} \mathrm{I}_{12}^{3}+2 . H_{11} \mathrm{I}_{23}^{2} \mathrm{I}_{11} \mathrm{I}_{22}^{2}$ $\mathrm{I}_{13}^{2} \mathrm{I}_{12}-4 . H_{22} \mathrm{I}_{13}^{3} \mathrm{I}_{11} \mathrm{I}_{22} \mathrm{I}_{12}^{2} \mathrm{I}_{23}+2 . H_{22} \mathrm{I}_{13}^{2} \mathrm{I}_{11}^{2} \mathrm{I}_{22} \mathrm{I}_{12} \mathrm{I}_{23}^{2}-2 . H_{22} \mathrm{I}_{13}^{2} \mathrm{I}_{11}^{2} \mathrm{I}_{22} \mathrm{I}_{12} \mathrm{I}_{33}+2 . H_{22} \mathrm{I}_{13}^{2} \mathrm{I}_{11} \mathrm{I}_{22} \mathrm{I}_{12}^{3} \mathrm{I}_{33}-2 . H_{22}$ $\mathrm{I}_{11}^{3} \mathrm{I}_{33} \mathrm{I}_{22} \mathrm{I}_{12} \mathrm{I}_{23}^{2}-4 . H_{22} \mathrm{I}_{11} \mathrm{I}_{33} \mathrm{I}_{12}^{4} \mathrm{I}_{23} \mathrm{I}_{13}-2 . H_{12} \mathrm{I}_{12}^{2} \mathrm{I}_{33} \mathrm{I}_{11}^{2} \mathrm{I}_{22} \mathrm{I}_{23}^{2}-2 . H_{12} \mathrm{I}_{12}^{2} \mathrm{I}_{33} \mathrm{I}_{11} \mathrm{I}_{22}^{2} \mathrm{I}_{13}^{2}-4 . H_{12} \mathrm{I}_{13}^{2} \mathrm{I}_{23}^{2} \mathrm{I}_{11} \mathrm{I}_{22} \mathrm{I}_{12}^{2}$

+ 4. $H_{12} \mathrm{I}_{13} \mathrm{I}_{23}^{3} \mathrm{I}_{11}^{2} \mathrm{I}_{22} \mathrm{I}_{12}+4 . H_{12} \mathrm{I}_{13}^{3} \mathrm{I}_{23} \mathrm{I}_{11} \mathrm{I}_{22}^{2} \mathrm{I}_{12}-2 . H_{11} \mathrm{I}_{22} \mathrm{I}_{33} \mathrm{I}_{11}^{2} \mathrm{I}_{12} \mathrm{I}_{23}^{2}-2 . H_{11} \mathrm{I}_{22}^{3} \mathrm{I}_{33} \mathrm{I}_{11} \mathrm{I}_{13}^{2} \mathrm{I}_{12}-4 . H_{11} \mathrm{I}_{22} \mathrm{I}_{33} \mathrm{I}_{12}^{4} \mathrm{I}_{23} \mathrm{I}_{13}$

+ 2. $H_{11} \mathrm{I}_{22} \mathrm{I}_{33} \mathrm{I}_{12}^{3} \mathrm{I}_{23}^{2} \mathrm{I}_{11}-4 . H_{11} \mathrm{I}_{23}^{3} \mathrm{I}_{11} \mathrm{I}_{22} \mathrm{I}_{12}^{2} \mathrm{I}_{13}+H_{22} \mathrm{I}_{11} \mathrm{I}_{33}^{2} \mathrm{I}_{12}^{\mathrm{S}}-1 . \mathrm{I}_{11}^{3} \mathrm{I}_{22}^{3} \mathrm{I}_{33}^{2} H_{12}-1 . \mathrm{I}_{11}^{3} \mathrm{I}_{23}^{4} H_{12} \mathrm{I}_{22}+\mathrm{I}_{11}^{3} \mathrm{I}_{23}^{4} H_{22} \mathrm{I}_{12}-1$.

$\mathrm{I}_{13}^{4} \mathrm{I}_{22}^{3} H_{12} \mathrm{I}_{11}+\mathrm{I}_{13}^{4} \mathrm{I}_{22}^{3} H_{11} \mathrm{I}_{12}+H_{11} \mathrm{I}_{22} \mathrm{I}_{33}^{2} \mathrm{I}_{12}^{5}+4 . H_{11} \mathrm{I}_{22}^{2} \mathrm{I}_{33} \mathrm{I}_{11} \mathrm{I}_{12}^{2} \mathrm{I}_{23} \mathrm{I}_{13}+4 . H_{22} \mathrm{I}_{11}^{2} \mathrm{I}_{33} \mathrm{I}_{22} \mathrm{I}_{12}^{2} \mathrm{I}_{23} \mathrm{I}_{13}+4 \cdot H_{12}$

$\mathrm{I}_{12}^{3} \mathrm{I}_{33} \mathrm{I}_{11} \mathrm{I}_{22} \mathrm{I}_{23} \mathrm{I}_{13}-4 . H_{12} \mathrm{I}_{13} \mathrm{I}_{23} \mathrm{I}_{11}^{2} \mathrm{I}_{22}^{2} \mathrm{I}_{12} \mathrm{I}_{33}$

dom $3:=p 33 \cdot \operatorname{simplify}\left(\left(p 22 \cdot p 33-p 23^{2}\right) \cdot\left(p 33 \cdot p 11-p 13^{2}\right)\right)$

$$
\left(\mathrm{I}_{11} \mathrm{I}_{22}-\mathrm{I}_{12}^{2}\right) \mathrm{I}_{11}\left(\mathrm{I}_{11} \mathrm{I}_{22} \mathrm{I}_{33}-\mathrm{I}_{11} \mathrm{I}_{23}^{2}-\mathrm{I}_{12}^{2} \mathrm{I}_{33}+2 \mathrm{I}_{12} \mathrm{I}_{13} \mathrm{I}_{23}-\mathrm{I}_{13}^{2} \mathrm{I}_{22}\right)^{2} \mathrm{I}_{22}
$$

$a_{1}:=\operatorname{simplify}\left(\frac{\Omega_{3}}{\operatorname{dom} 3}\right)$

$$
-\frac{1 .\left(\mathrm{I}_{22} H_{12} \mathrm{I}_{11}-1 . \mathrm{I}_{22} H_{11} \mathrm{I}_{12}-1 . H_{22} \mathrm{I}_{12} \mathrm{I}_{11}\right)}{\mathrm{I}_{22} \mathrm{I}_{11}\left(\mathrm{I}_{11} \mathrm{I}_{22}-1 . \mathrm{I}_{12}^{2}\right)}
$$




\section{A.3 Even expressions}


solve $\left(\left\{\alpha_{1}^{2}=\mathrm{I}_{11}, \alpha_{1} \cdot \beta_{1}=h I_{11}, \alpha_{1} \cdot \alpha_{2}=\mathrm{I}_{12}, \alpha_{1} \cdot \beta_{2}+\beta_{1} \cdot \alpha_{2}=h I_{12}, \alpha_{2}^{2}+\alpha_{3}^{2}=\mathrm{I}_{22}, \alpha_{2} \cdot \beta_{2}+\beta_{3} \cdot \alpha_{3}=h I_{22}, \alpha_{1} \cdot \alpha_{4}=\mathrm{I}_{13}, \alpha_{1} \cdot \beta_{4}+\beta_{1} \cdot \alpha_{4}=h I_{13}, \alpha_{2} \cdot \alpha_{4}\right.\right.$ $\left.+\alpha_{3} \cdot \alpha_{5}=I_{23}, \alpha_{2} \cdot \beta_{4}+\alpha_{3} \cdot \beta_{5}+\alpha_{4} \cdot \beta_{2}+\alpha_{5} \cdot \beta_{3}=h I_{23}, \alpha_{4} \cdot \beta_{4}+\alpha_{5} \cdot \beta_{5}+\alpha_{6} \cdot \beta_{6}=h I_{33}, \alpha_{4}^{2}+\alpha_{5}^{2}+\alpha_{6}^{2}=I_{33}\right\},\left\{\alpha_{1}, \alpha_{2}, \alpha_{3}, \alpha_{4}, \alpha_{5}, \alpha_{6}, \beta_{1}\right.$,

$\left.\left.\beta_{2}, \beta_{3}, \beta_{4}, \beta_{5}, \beta_{6}\right\}\right)$;

$\left\{\alpha_{1}=\operatorname{RootOf}\left(Z^{2}-\mathrm{I}_{11}\right), \alpha_{2}=\frac{\mathrm{I}_{12} \operatorname{RootOf}\left(Z^{2}-\mathrm{I}_{11}\right)}{\mathrm{I}_{11}}, \alpha_{3}=\operatorname{RootOf}\left(\mathrm{I}_{12}^{2}+\mathrm{I}_{11} Z^{2}-\mathrm{I}_{11} \mathrm{I}_{22}\right), \alpha_{4}=\frac{\mathrm{I}_{13} \operatorname{RootOf}\left(Z^{2}-\mathrm{I}_{11}\right)}{\mathrm{I}_{11}}, \alpha_{5}\right.$

$=\frac{-\mathrm{I}_{12} \mathrm{I}_{13}+\mathrm{I}_{11} \mathrm{I}_{23}}{\mathrm{I}_{11} \operatorname{RootOf}\left(\mathrm{I}_{12}^{2}+\mathrm{I}_{11} Z^{2}-\mathrm{I}_{11} \mathrm{I}_{22}\right)}, \alpha_{6}=\operatorname{RootOf}\left(\left(-\mathrm{I}_{12}^{2}+\mathrm{I}_{11} \mathrm{I}_{22}\right) Z^{2}-2 \mathrm{I}_{12} \mathrm{I}_{13} \mathrm{I}_{23}+\mathrm{I}_{11} \mathrm{I}_{23}^{2}-\mathrm{I}_{11} \mathrm{I}_{22} \mathrm{I}_{33}+\mathrm{I}_{12}^{2} \mathrm{I}_{33}+\mathrm{I}_{22}\right.$

$\left.\mathrm{I}_{13}^{2}\right), \beta_{1}=\frac{h I_{11} \operatorname{RootOf}\left(-Z^{2}-\mathrm{I}_{11}\right)}{\mathrm{I}_{11}}, \beta_{2}=\frac{\left(-h I_{11} \mathrm{I}_{12}+h I_{12} \mathrm{I}_{11}\right) \operatorname{RootOf}\left(-Z^{2}-\mathrm{I}_{11}\right)}{\mathrm{I}_{11}^{2}}, \beta_{3}=\frac{h I_{11} I_{12}^{2}-\mathrm{I}_{12} h I_{12} \mathrm{I}_{11}+h I_{22} \mathrm{I}_{11}^{2}}{\mathrm{I}_{11}^{2} \operatorname{RootOf}\left(\mathrm{I}_{12}^{2}+\mathrm{I}_{11} Z^{2}-\mathrm{I}_{11} \mathrm{I}_{22}\right)}$,

$\beta_{4}=\frac{\left(-h I_{11} \mathrm{I}_{13}+h I_{13} \mathrm{I}_{11}\right) \operatorname{RootOf}\left(Z^{2}-\mathrm{I}_{11}\right)}{\mathrm{I}_{11}^{2}}, \beta_{5}=\frac{1}{\mathrm{I}_{11}^{2} \operatorname{RootOf}\left(\mathrm{I}_{12}^{2}+\mathrm{I}_{11} Z^{2}-\mathrm{I}_{11} \mathrm{I}_{22}\right)\left(-\mathrm{I}_{12}^{2}+\mathrm{I}_{11} \mathrm{I}_{22}\right)}\left(-h I_{11} \mathrm{I}_{12}^{3} \mathrm{I}_{13}\right.$

$-h I_{11} \mathrm{I}_{12}^{2} \mathrm{I}_{11} \mathrm{I}_{23}+\mathrm{I}_{12} h I_{12} \mathrm{I}_{11}^{2} \mathrm{I}_{23}+h I_{22} \mathrm{I}_{11}^{2} \mathrm{I}_{12} \mathrm{I}_{13}-h I_{22} \mathrm{I}_{11}^{3} \mathrm{I}_{23}-\mathrm{I}_{11}^{2} \mathrm{I}_{22} \mathrm{I}_{13} h I_{12}+\mathrm{I}_{11}^{3} \mathrm{I}_{22} h I_{23}-\mathrm{I}_{12}^{2} h I_{23} \mathrm{I}_{11}^{2}+\mathrm{I}_{12}^{3} \mathrm{I}_{11} h I_{13}$

$\left.+2 \mathrm{I}_{11} \mathrm{I}_{22} \mathrm{I}_{12} h I_{11} \mathrm{I}_{13}-\mathrm{I}_{11}^{2} \mathrm{I}_{22} \mathrm{I}_{12} h I_{13}\right), \beta_{6}=\left(\mathrm{I}_{12}^{2} \mathrm{I}_{13} \mathrm{I}_{22} h I_{13}+\mathrm{I}_{12}^{2} \mathrm{I}_{13} h I_{12} \mathrm{I}_{23}-2 \mathrm{I}_{12} \mathrm{I}_{13} h I_{22} \mathrm{I}_{11} \mathrm{I}_{23}-\mathrm{I}_{12} \mathrm{I}_{13}^{2} \mathrm{I}_{22} h I_{12}\right.$

$+\mathrm{I}_{12} \mathrm{I}_{13} \mathrm{I}_{11} \mathrm{I}_{22} h I_{23}+\mathrm{I}_{11} \mathrm{I}_{23} \mathrm{I}_{22} \mathrm{I}_{13} h I_{12}+\mathrm{I}_{11} \mathrm{I}_{23} \mathrm{I}_{22} \mathrm{I}_{12} h I_{13}-2 \mathrm{I}_{12}^{2} h I_{33} \mathrm{I}_{11} \mathrm{I}_{22}+\mathrm{I}_{22}^{2} h I_{11} \mathrm{I}_{13}^{2}-\mathrm{I}_{11} \mathrm{I}_{22}^{2} \mathrm{I}_{13} h I_{13}+\mathrm{I}_{12}^{2} \mathrm{I}_{13}^{2} h I_{22}-$

$\mathrm{I}_{12}^{3} \mathrm{I}_{13} h I_{23}+\mathrm{I}_{23}^{2} h I_{11} \mathrm{I}_{12}^{2}-\mathrm{I}_{11} \mathrm{I}_{23}^{2} \mathrm{I}_{12} h I_{12}-\mathrm{I}_{11}^{2} \mathrm{I}_{23} \mathrm{I}_{22} h I_{23}+\mathrm{I}_{11} \mathrm{I}_{23} \mathrm{I}_{12}^{2} h I_{23}-\mathrm{I}_{23} \mathrm{I}_{12}^{3} h I_{13}+\mathrm{I}_{12}^{4} h I_{33}+\mathrm{I}_{11}^{2} I_{22}^{2} h I_{33}+\mathrm{I}_{11}^{2} \mathrm{I}_{23}^{2} h I_{22}$

$\left.-2 \mathrm{I}_{23} \mathrm{I}_{22} \mathrm{I}_{12} h \mathrm{I}_{11} \mathrm{I}_{13}\right) /\left(\operatorname{RootOf}\left(\left(-\mathrm{I}_{12}^{2}+\mathrm{I}_{11} \mathrm{I}_{22}\right) Z^{2}-2 \mathrm{I}_{12} \mathrm{I}_{13} \mathrm{I}_{23}+\mathrm{I}_{11} \mathrm{I}_{23}^{2}-\mathrm{I}_{11} \mathrm{I}_{22} \mathrm{I}_{33}+\mathrm{I}_{12}^{2} \mathrm{I}_{33}+\mathrm{I}_{22} \mathrm{I}_{13}^{2}\right)\left(-2 \mathrm{I}_{12}^{2} \mathrm{I}_{11} \mathrm{I}_{22}+\right.\right.$

$\left.\left.\left.\mathrm{I}_{12}^{4}+\mathrm{I}_{11}^{2} \mathrm{I}_{22}^{2}\right)\right)\right\}$

$\alpha_{1}:=\operatorname{RootOf}\left(Z^{2}-\mathrm{I}_{11}\right) ; \alpha_{2}:=\frac{\mathrm{I}_{12} \operatorname{RootOf}\left(Z^{2}-\mathrm{I}_{11}\right)}{\mathrm{I}_{11}} ; \alpha_{3}:=\operatorname{RootOf}\left(\mathrm{I}_{12}^{2}+\mathrm{I}_{11} Z^{2}-\mathrm{I}_{11} \mathrm{I}_{22}\right) ; \alpha_{4}:=\frac{\mathrm{I}_{13} \operatorname{RootOf}\left(Z^{2}-\mathrm{I}_{11}\right)}{\mathrm{I}_{11}} ; \alpha_{5}$

$:=\frac{-\mathrm{I}_{12} \mathrm{I}_{13}+\mathrm{I}_{11} \mathrm{I}_{23}}{\mathrm{I}_{11} \operatorname{RootOf}\left(\mathrm{I}_{12}^{2}+\mathrm{I}_{11} Z^{2}-\mathrm{I}_{11} \mathrm{I}_{22}\right)} ; \alpha_{6}:=\operatorname{RootOf}\left(\left(-\mathrm{I}_{12}^{2}+\mathrm{I}_{11} \mathrm{I}_{22}\right) Z^{2}-2 \mathrm{I}_{12} \mathrm{I}_{13} \mathrm{I}_{23}+\mathrm{I}_{11} \mathrm{I}_{23}^{2}-\mathrm{I}_{11} \mathrm{I}_{22} \mathrm{I}_{33}+\mathrm{I}_{12}^{2} \mathrm{I}_{33}+\mathrm{I}_{22} \mathrm{I}_{13}^{2}\right) ; \beta_{1}$

$:=\frac{h I_{11} \operatorname{RootOf}\left({ }_{-} Z^{2}-\mathrm{I}_{11}\right)}{\mathrm{I}_{11}} ; \beta_{2}:=\frac{\left(-h I_{11} \mathrm{I}_{12}+h I_{12} \mathrm{I}_{11}\right) \operatorname{RootOf}\left(Z^{2}-\mathrm{I}_{11}\right)}{\mathrm{I}_{11}^{2}} ; \beta_{3}:=\frac{h I_{11} \mathrm{I}_{12}^{2}-\mathrm{I}_{12} h I_{12} \mathrm{I}_{11}+h I_{22} \mathrm{I}_{11}^{2}}{\mathrm{I}_{11}^{2} \operatorname{RootOf}\left(\mathrm{I}_{12}^{2}+\mathrm{I}_{11} Z^{2}-\mathrm{I}_{11} \mathrm{I}_{22}\right)} ; \beta_{4}$

$:=\frac{\left(-h I_{11} \mathrm{I}_{13}+h I_{13} \mathrm{I}_{11}\right) \operatorname{RootOf}\left(-Z^{2}-\mathrm{I}_{11}\right)}{\mathrm{I}_{11}^{2}} ; \beta_{5}:=\frac{1}{\mathrm{I}_{11}^{2} \operatorname{RootOf}\left(\mathrm{I}_{12}^{2}+\mathrm{I}_{11} Z^{2}-\mathrm{I}_{11} \mathrm{I}_{22}\right)\left(-\mathrm{I}_{12}^{2}+\mathrm{I}_{11} \mathrm{I}_{22}\right)}\left(-h I_{11} \mathrm{I}_{12}^{3} \mathrm{I}_{13}-h I_{11}\right.$

$\mathrm{I}_{12}^{2} \mathrm{I}_{11} \mathrm{I}_{23}+\mathrm{I}_{12} h I_{12} \mathrm{I}_{11}^{2} \mathrm{I}_{23}+h I_{22} \mathrm{I}_{11}^{2} \mathrm{I}_{12} \mathrm{I}_{13}-h I_{22} \mathrm{I}_{11}^{3} \mathrm{I}_{23}-\mathrm{I}_{11}^{2} \mathrm{I}_{22} \mathrm{I}_{13} h I_{12}+\mathrm{I}_{11}^{3} \mathrm{I}_{22} h I_{23}-\mathrm{I}_{12}^{2} h I_{23} \mathrm{I}_{11}^{2}+\mathrm{I}_{12}^{3} \mathrm{I}_{11} h I_{13}+2 \mathrm{I}_{11} \mathrm{I}_{22} \mathrm{I}_{12} h I_{11} \mathrm{I}_{13}$

$\left.-\mathrm{I}_{11}^{2} \mathrm{I}_{22} \mathrm{I}_{12} h I_{13}\right) ; \beta_{6}:=\left(\mathrm{I}_{11} \mathrm{I}_{23} \mathrm{I}_{22} \mathrm{I}_{12} h I_{13}+\mathrm{I}_{12}^{2} \mathrm{I}_{13} \mathrm{I}_{22} h I_{13}+\mathrm{I}_{12}^{2} \mathrm{I}_{13} h I_{12} \mathrm{I}_{23}-2 \mathrm{I}_{12} \mathrm{I}_{13} h I_{22} \mathrm{I}_{11} \mathrm{I}_{23}-\mathrm{I}_{12} \mathrm{I}_{13}^{2} \mathrm{I}_{22} h I_{12}\right.$

$+\mathrm{I}_{12} \mathrm{I}_{13} \mathrm{I}_{11} \mathrm{I}_{22} h I_{23}+\mathrm{I}_{11} \mathrm{I}_{23} \mathrm{I}_{22} \mathrm{I}_{13} h I_{12}-2 \mathrm{I}_{12}^{2} h I_{33} \mathrm{I}_{11} \mathrm{I}_{22}+\mathrm{I}_{22}^{2} h I_{11} \mathrm{I}_{13}^{2}-\mathrm{I}_{11} \mathrm{I}_{22}^{2} \mathrm{I}_{13} h I_{13}+\mathrm{I}_{12}^{2} \mathrm{I}_{13}^{2} h I_{22}-\mathrm{I}_{12}^{3} \mathrm{I}_{13} h I_{23}+\mathrm{I}_{23}^{2} h I_{11} \mathrm{I}_{12}^{2}-\mathrm{I}_{11}$

$\left.\mathrm{I}_{23}^{2} \mathrm{I}_{12} h I_{12}-\mathrm{I}_{11}^{2} \mathrm{I}_{23} \mathrm{I}_{22} h I_{23}+\mathrm{I}_{11} \mathrm{I}_{23} \mathrm{I}_{12}^{2} h I_{23}-\mathrm{I}_{23} \mathrm{P}_{12}^{3} h I_{13}+\mathrm{I}_{12}^{4} h I_{33}+\mathrm{I}_{11}^{2} \mathrm{I}_{22}^{2} h I_{33}+\mathrm{I}_{11}^{2} \mathrm{I}_{23}^{2} h I_{22}-2 \mathrm{I}_{23} \mathrm{I}_{22} \mathrm{I}_{12} h I_{11} \mathrm{I}_{13}\right) /\left(\right.$ RootOf $\left(\left(-\mathrm{I}_{12}^{2}\right.\right.$

$\left.\left.\left.+\mathrm{I}_{11} \mathrm{I}_{22}\right) Z^{2}-2 \mathrm{I}_{12} \mathrm{I}_{13} \mathrm{I}_{23}+\mathrm{I}_{11} \mathrm{I}_{23}^{2}-\mathrm{I}_{11} \mathrm{I}_{22} \mathrm{I}_{33}+\mathrm{I}_{12}^{2} \mathrm{I}_{33}+\mathrm{I}_{22} \mathrm{I}_{13}^{2}\right)\left(-2 \mathrm{I}_{12}^{2} \mathrm{I}_{11} \mathrm{I}_{22}+\mathrm{I}_{12}^{4}+\mathrm{I}_{11}^{2} \mathrm{I}_{22}^{2}\right)\right)$

$$
\begin{gathered}
\frac{\operatorname{RootOf}\left(Z^{2}-\mathrm{I}_{11}\right)}{\mathrm{I}_{12} \operatorname{RootOf}\left(-Z^{2}-\mathrm{I}_{11}\right)} \\
\mathrm{I}_{11} \\
\operatorname{RootOf}\left(\mathrm{I}_{12}^{2}+\mathrm{I}_{11} Z^{2}-\mathrm{I}_{11} \mathrm{I}_{22}\right) \\
\frac{\mathrm{I}_{13} \operatorname{RootOf}\left(Z^{2}-\mathrm{I}_{11}\right)}{\mathrm{I}_{11}} \\
\frac{-\mathrm{I}_{12} \mathrm{I}_{13}+\mathrm{I}_{11} \mathrm{I}_{23}}{\mathrm{I}_{11} \operatorname{RootOf}\left(\mathrm{I}_{12}^{2}+\mathrm{I}_{11} Z^{2}-\mathrm{I}_{11} \mathrm{I}_{22}\right)} \\
\frac{h I_{11} \operatorname{Root} O f\left(Z^{2}-\mathrm{I}_{11}\right)}{\mathrm{I}_{11}}
\end{gathered}
$$




$$
\begin{gathered}
\frac{\left(-h I_{11} \mathrm{I}_{12}+h I_{12} \mathrm{I}_{11}\right) \operatorname{Root} O f\left(-Z^{2}-\mathrm{I}_{11}\right)}{\mathrm{I}_{11}^{2}} \\
\frac{h I_{11} \mathrm{I}_{12}^{2}-\mathrm{I}_{12} h I_{12} \mathrm{I}_{11}+h I_{22} \mathrm{I}_{11}^{2}}{\mathrm{I}_{11}^{2} \operatorname{RootOf}\left(\mathrm{I}_{12}^{2}+\mathrm{I}_{11} Z^{2}-\mathrm{I}_{11} \mathrm{I}_{22}\right)} \\
\frac{\left(-h I_{11} \mathrm{I}_{13}+h I_{13} \mathrm{I}_{11}\right) \operatorname{RootOf}\left(-Z^{2}-\mathrm{I}_{11}\right)}{\mathrm{I}_{11}^{2}}
\end{gathered}
$$

$\frac{1}{\mathrm{I}_{11}^{2} \operatorname{RootOf}\left(\mathrm{I}_{12}^{2}+\mathrm{I}_{11} Z^{2}-\mathrm{I}_{11} \mathrm{I}_{22}\right)\left(-\mathrm{I}_{12}^{2}+\mathrm{I}_{11} \mathrm{I}_{22}\right)}\left(-h I_{11} \mathrm{P}_{12}^{3} \mathrm{I}_{13}-h I_{11} \mathrm{I}_{12}^{2} \mathrm{I}_{11} \mathrm{I}_{23}+\mathrm{I}_{12} h I_{12} \mathrm{I}_{11}^{2} \mathrm{I}_{23}+h I_{22} \mathrm{I}_{11}^{2} \mathrm{I}_{12} \mathrm{I}_{13}-h I_{22} \mathrm{I}_{11}^{3} \mathrm{I}_{23}-\right.$

$\left.\mathrm{I}_{11}^{2} \mathrm{I}_{22} \mathrm{I}_{13} h I_{12}+\mathrm{I}_{11}^{3} \mathrm{I}_{22} h I_{23}-\mathrm{I}_{12}^{2} h I_{23} \mathrm{I}_{11}^{2}+\mathrm{I}_{12}^{3} \mathrm{I}_{11} h I_{13}+2 \mathrm{I}_{11} \mathrm{I}_{22} \mathrm{I}_{12} h I_{11} \mathrm{I}_{13}-\mathrm{I}_{11}^{2} \mathrm{I}_{22} \mathrm{I}_{12} h I_{13}\right)$

$\left(\mathrm{I}_{12}^{2} \mathrm{I}_{13} \mathrm{I}_{22} h I_{13}+\mathrm{I}_{12}^{2} \mathrm{I}_{13} h I_{12} \mathrm{I}_{23}-2 \mathrm{I}_{12} \mathrm{I}_{13} h I_{22} \mathrm{I}_{11} \mathrm{I}_{23}-\mathrm{I}_{12} \mathrm{I}_{13}^{2} \mathrm{I}_{22} h I_{12}+\mathrm{I}_{12} \mathrm{I}_{13} \mathrm{I}_{11} \mathrm{I}_{22} h I_{23}+\mathrm{I}_{11} \mathrm{I}_{23} \mathrm{I}_{22} \mathrm{I}_{13} h I_{12}+\mathrm{I}_{11} \mathrm{I}_{23} \mathrm{I}_{22} \mathrm{I}_{12} h I_{13}\right.$

(2)

$-2 \mathrm{I}_{12}^{2} h I_{33} \mathrm{I}_{11} \mathrm{I}_{22}+\mathrm{I}_{22}^{2} h I_{11} \mathrm{I}_{13}^{2}-\mathrm{I}_{11} \mathrm{I}_{22}^{2} \mathrm{I}_{13} h I_{13}+\mathrm{I}_{12}^{2} \mathrm{I}_{13}^{2} h I_{22}-\mathrm{I}_{12}^{3} \mathrm{I}_{13} h I_{23}+\mathrm{I}_{23}^{2} h I_{11} \mathrm{I}_{12}^{2}-\mathrm{I}_{11} \mathrm{I}_{23}^{2} \mathrm{I}_{12} h I_{12}-\mathrm{I}_{11}^{2} \mathrm{I}_{23} \mathrm{I}_{22} h I_{23}$

$\left.+\mathrm{I}_{11} \mathrm{I}_{23} \mathrm{I}_{12}^{2} h I_{23}-\mathrm{I}_{23} \mathrm{I}_{12}^{3} h I_{13}+\mathrm{I}_{12}^{4} h I_{33}+\mathrm{I}_{11}^{2} \mathrm{I}_{22}^{2} h I_{33}+\mathrm{I}_{11}^{2} \mathrm{I}_{23}^{2} h I_{22}-2 \mathrm{I}_{23} \mathrm{I}_{22} \mathrm{I}_{12} h I_{11} \mathrm{I}_{13}\right) /\left(\operatorname{RootOf}\left(\left(-\mathrm{I}_{12}^{2}+\mathrm{I}_{11} \mathrm{I}_{22}\right) Z^{2}\right.\right.$

$\left.\left.-2 \mathrm{I}_{12} \mathrm{I}_{13} \mathrm{I}_{23}+\mathrm{I}_{11} \mathrm{I}_{23}^{2}-\mathrm{I}_{11} \mathrm{I}_{22} \mathrm{I}_{33}+\mathrm{I}_{12}^{2} \mathrm{I}_{33}+\mathrm{I}_{22} \mathrm{I}_{13}^{2}\right)\left(-2 \mathrm{I}_{12}^{2} \mathrm{I}_{11} \mathrm{I}_{22}+\mathrm{I}_{12}^{4}+\mathrm{I}_{11}^{2} \mathrm{I}_{22}^{2}\right)\right)$

$\left[>j_{1}:=\operatorname{simplify}\left(\mathrm{\alpha}_{6}^{2}\right)\right.$;

$$
j_{1}:=\frac{2 \mathrm{I}_{12} \mathrm{I}_{13} \mathrm{I}_{23}-\mathrm{I}_{11} \mathrm{I}_{23}^{2}+\mathrm{I}_{11} \mathrm{I}_{22} \mathrm{I}_{33}-\mathrm{I}_{12}^{2} \mathrm{I}_{33}-\mathrm{I}_{22} \mathrm{I}_{13}^{2}}{-\mathrm{I}_{12}^{2}+\mathrm{I}_{11} \mathrm{I}_{22}}
$$

$j_{2}:=\operatorname{simplify}\left(\beta_{6}^{2}\right) ;$

$\left(\mathrm{I}_{12}^{2} \mathrm{I}_{13} \mathrm{I}_{22} h I_{13}+\mathrm{I}_{12}^{2} \mathrm{I}_{13} h I_{12} \mathrm{I}_{23}-2 \mathrm{I}_{12} \mathrm{I}_{13} h I_{22} \mathrm{I}_{11} \mathrm{I}_{23}-\mathrm{I}_{12} \mathrm{I}_{13}^{2} \mathrm{I}_{22} h I_{12}+\mathrm{I}_{12} \mathrm{I}_{13} \mathrm{I}_{11} \mathrm{I}_{22} h I_{23}+\mathrm{I}_{11} \mathrm{I}_{23} \mathrm{I}_{22} \mathrm{I}_{13} h I_{12}+\mathrm{I}_{11} \mathrm{I}_{23} \mathrm{I}_{22} \mathrm{I}_{12} h I_{13}\right.$

$-2 \mathrm{I}_{12}^{2} h I_{33} \mathrm{I}_{11} \mathrm{I}_{22}+\mathrm{I}_{22}^{2} h I_{11} \mathrm{I}_{13}^{2}-\mathrm{I}_{11} \mathrm{I}_{22}^{2} \mathrm{I}_{13} h I_{13}+\mathrm{I}_{12}^{2} \mathrm{I}_{13}^{2} h I_{22}-\mathrm{I}_{12}^{3} \mathrm{I}_{13} h I_{23}+\mathrm{I}_{23}^{2} h I_{11} \mathrm{I}_{12}^{2}-\mathrm{I}_{11} \mathrm{I}_{23}^{2} \mathrm{I}_{12} h I_{12}-\mathrm{I}_{11}^{2} \mathrm{I}_{23} \mathrm{I}_{22} h I_{23}$

$\left.+\mathrm{I}_{11} \mathrm{I}_{23} \mathrm{I}_{12}^{2} h I_{23}-\mathrm{I}_{23} \mathrm{I}_{12}^{3} h I_{13}+\mathrm{I}_{12}^{4} h I_{33}+\mathrm{I}_{11}^{2} \mathrm{I}_{22}^{2} h I_{33}+\mathrm{I}_{11}^{2} \mathrm{I}_{23}^{2} h I_{22}-2 \mathrm{I}_{23} \mathrm{I}_{22} \mathrm{I}_{12} h I_{11} \mathrm{I}_{13}\right)^{2} /\left(\left(-\mathrm{I}_{12}^{2}+\mathrm{I}_{11} \mathrm{I}_{22}\right)\left(-2 \mathrm{I}_{12}^{2} \mathrm{I}_{11} \mathrm{I}_{22}\right.\right.$

$\left.\left.+\mathrm{I}_{12}^{4}+\mathrm{I}_{11}^{2} \mathrm{I}_{22}^{2}\right)\left(2 \mathrm{I}_{12} \mathrm{I}_{13} \mathrm{I}_{23}-\mathrm{I}_{11} \mathrm{I}_{23}^{2}+\mathrm{I}_{11} \mathrm{I}_{22} \mathrm{I}_{33}-\mathrm{I}_{12}^{2} \mathrm{I}_{33}-\mathrm{I}_{22} \mathrm{I}_{13}^{2}\right)\right)$

$j_{3}:=\operatorname{simplify}\left(\alpha_{6} \cdot \beta_{6}\right)$;

$\frac{1}{-2 \mathrm{I}_{12}^{2} \mathrm{I}_{11} \mathrm{I}_{22}+\mathrm{I}_{12}^{4}+\mathrm{I}_{11}^{2} \mathrm{I}_{22}^{2}}\left(\mathrm{I}_{12}^{2} \mathrm{I}_{13} \mathrm{I}_{22} h I_{13}+\mathrm{I}_{12}^{2} \mathrm{I}_{13} h I_{12} \mathrm{I}_{23}-2 \mathrm{I}_{12} \mathrm{I}_{13} h I_{22} \mathrm{I}_{11} \mathrm{I}_{23}-\mathrm{I}_{12} \mathrm{I}_{13}^{2} \mathrm{I}_{22} h I_{12}+\mathrm{I}_{12} \mathrm{I}_{13} \mathrm{I}_{11} \mathrm{I}_{22} h I_{23}\right.$

$+\mathrm{I}_{11} \mathrm{I}_{23} \mathrm{I}_{22} \mathrm{I}_{13} h I_{12}+\mathrm{I}_{11} \mathrm{I}_{23} \mathrm{I}_{22} \mathrm{I}_{12} h I_{13}-2 \mathrm{I}_{12}^{2} h I_{33} \mathrm{I}_{11} \mathrm{I}_{22}+\mathrm{I}_{22}^{2} h I_{11} \mathrm{I}_{13}^{2}-\mathrm{I}_{11} \mathrm{I}_{22}^{2} \mathrm{I}_{13} h I_{13}+\mathrm{I}_{12}^{2} \mathrm{I}_{13}^{2} h I_{22}-\mathrm{I}_{12}^{3} \mathrm{I}_{13} h I_{23}+\mathrm{I}_{23}^{2} h I_{11}$

$\left.\mathrm{I}_{12}^{2}-\mathrm{I}_{11} \mathrm{I}_{23}^{2} \mathrm{I}_{12} h I_{12}-\mathrm{I}_{11}^{2} \mathrm{I}_{23} \mathrm{I}_{22} h I_{23}+\mathrm{I}_{11} \mathrm{I}_{23} \mathrm{I}_{12}^{2} h I_{23}-\mathrm{I}_{23} \mathrm{I}_{12}^{3} h I_{13}+\mathrm{I}_{12}^{4} h I_{33}+\mathrm{I}_{11}^{2} \mathrm{I}_{22}^{2} h I_{33}+\mathrm{I}_{11}^{2} \mathrm{I}_{23}^{2} h I_{22}-2 \mathrm{I}_{23} \mathrm{I}_{22} \mathrm{I}_{12} h I_{11} \mathrm{I}_{13}\right)$ $j_{4}:=\operatorname{simplify}\left(\alpha_{3}^{2}\right)$;

$$
\frac{-\mathrm{I}_{12}^{2}+\mathrm{I}_{11} \mathrm{I}_{22}}{\mathrm{I}_{11}}
$$

$j_{5}:=\operatorname{simplify}\left(\alpha_{5}^{2}\right)$

$$
\frac{\left(-I_{12} I_{13}+I_{11} I_{23}\right)^{2}}{\left(-I_{12}^{2}+I_{11} I_{22}\right) I_{11}}
$$

$j_{6}:=\operatorname{simplify}\left(\alpha_{3} \cdot \alpha_{5}\right) ;$

$$
\frac{-\mathrm{I}_{12} \mathrm{I}_{13}+\mathrm{I}_{11} \mathrm{I}_{23}}{\mathrm{I}_{11}}
$$

$j_{7}:=\operatorname{simplify}\left(\beta_{3}^{2}\right) ;$

$$
\frac{\left(h I_{11} \mathrm{I}_{12}^{2}-\mathrm{I}_{12} h I_{12} \mathrm{I}_{11}+h I_{22} \mathrm{I}_{11}^{2}\right)^{2}}{\left(-\mathrm{I}_{12}^{2}+\mathrm{I}_{11} \mathrm{I}_{22}\right) \mathrm{I}_{11}^{3}}
$$

$j_{8}:=\operatorname{simplify}\left(\beta_{5}^{2}\right)$ 


$$
\begin{aligned}
& \frac{1}{\left(-\mathrm{I}_{12}^{2}+\mathrm{I}_{11} \mathrm{I}_{22}\right)^{3} \mathrm{I}_{11}^{3}}\left(-h I_{11} \mathrm{I}_{12}^{3} \mathrm{I}_{13}-h I_{11} \mathrm{I}_{12}^{2} \mathrm{I}_{11} \mathrm{I}_{23}+\mathrm{I}_{12} h I_{12} \mathrm{I}_{11}^{2} \mathrm{I}_{23}+h I_{22} \mathrm{I}_{11}^{2} \mathrm{I}_{12} \mathrm{I}_{13}-h I_{22} \mathrm{I}_{11}^{3} \mathrm{I}_{23}-\mathrm{I}_{11}^{2} \mathrm{I}_{22} \mathrm{I}_{13} h I_{12}+\mathrm{I}_{11}^{3} \mathrm{I}_{22} h I_{23}-\right. \\
& \left.\mathrm{I}_{12}^{2} h I_{23} \mathrm{I}_{11}^{2}+\mathrm{I}_{12}^{3} \mathrm{I}_{11} h I_{13}+2 \mathrm{I}_{11} \mathrm{I}_{22} \mathrm{I}_{12} h I_{11} \mathrm{I}_{13}-\mathrm{I}_{11}^{2} \mathrm{I}_{22} \mathrm{I}_{12} h I_{13}\right)^{2}
\end{aligned}
$$

$j_{9}:=\operatorname{simplify}\left(\beta_{3} \cdot \beta_{5}\right)$;

$$
\begin{aligned}
& \frac{1}{\mathrm{I}_{11}^{3}\left(-\mathrm{I}_{12}^{2}+\mathrm{I}_{11} \mathrm{I}_{22}\right)^{2}}\left(( h I _ { 1 1 } \mathrm { I } _ { 1 2 } ^ { 2 } - \mathrm { I } _ { 1 2 } h I _ { 1 2 } \mathrm { I } _ { 1 1 } + h I _ { 2 2 } \mathrm { I } _ { 1 1 } ^ { 2 } ) \left(-h I_{11} \mathrm{I}_{12}^{3} \mathrm{I}_{13}-h I_{11} \mathrm{I}_{12}^{2} \mathrm{I}_{11} \mathrm{I}_{23}+\mathrm{I}_{12} h I_{12} \mathrm{I}_{11}^{2} \mathrm{I}_{23}+h I_{22} \mathrm{I}_{11}^{2} \mathrm{I}_{12} \mathrm{I}_{13}-h I_{22}\right.\right. \\
& \left.\left.\mathrm{I}_{11}^{3} \mathrm{I}_{23}-\mathrm{I}_{11}^{2} \mathrm{I}_{22} \mathrm{I}_{13} h I_{12}+\mathrm{I}_{11}^{3} \mathrm{I}_{22} h I_{23}-\mathrm{I}_{12}^{2} h I_{23} \mathrm{I}_{11}^{2}+\mathrm{I}_{12}^{3} \mathrm{I}_{11} h I_{13}+2 \mathrm{I}_{11} \mathrm{I}_{22} \mathrm{I}_{12} h I_{11} \mathrm{I}_{13}-\mathrm{I}_{11}^{2} \mathrm{I}_{22} \mathrm{I}_{12} h I_{13}\right)\right) \\
& j_{10}:=\operatorname{simplify}\left(\alpha_{5} \cdot \mathrm{\beta}_{5}\right) ; \\
& \frac{1}{\left(-\mathrm{I}_{12}^{2}+\mathrm{I}_{11} \mathrm{I}_{22}\right)^{2} \mathrm{I}_{11}^{2}}\left(( - \mathrm { I } _ { 1 2 } \mathrm { I } _ { 1 3 } + \mathrm { I } _ { 1 1 } \mathrm { I } _ { 2 3 } ) \left(-h I_{11} \mathrm{I}_{12}^{3} \mathrm{I}_{13}-h I_{11} \mathrm{I}_{12}^{2} \mathrm{I}_{11} \mathrm{I}_{23}+\mathrm{I}_{12} h I_{12} \mathrm{I}_{11}^{2} \mathrm{I}_{23}+h I_{22} \mathrm{I}_{11}^{2} \mathrm{I}_{12} \mathrm{I}_{13}-h I_{22} \mathrm{I}_{11}^{3} \mathrm{I}_{23}-\right.\right. \\
& \left.\left.\mathrm{I}_{11}^{2} \mathrm{I}_{22} \mathrm{I}_{13} h I_{12}+\mathrm{I}_{11}^{3} \mathrm{I}_{22} h I_{23}-\mathrm{I}_{12}^{2} h I_{23} \mathrm{I}_{11}^{2}+\mathrm{I}_{12}^{3} \mathrm{I}_{11} h I_{13}+2 \mathrm{I}_{11} \mathrm{I}_{22} \mathrm{I}_{12} h I_{11} \mathrm{I}_{13}-\mathrm{I}_{11}^{2} \mathrm{I}_{22} \mathrm{I}_{12} h I_{13}\right)\right) \\
& j_{11}:=\operatorname{simplify}\left(\alpha_{3} \cdot \beta_{3}\right) ; \\
& \frac{h I_{11} \mathrm{I}_{12}^{2}-\mathrm{I}_{12} h I_{12} \mathrm{I}_{11}+h I_{22} \mathrm{I}_{11}^{2}}{\mathrm{I}_{11}^{2}}
\end{aligned}
$$

$j_{12}=\operatorname{simplify}\left(\alpha_{3} \cdot \beta_{5}\right) ;$

$$
\begin{aligned}
& j_{12}=\frac{1}{\mathrm{I}_{11}^{2}\left(-\mathrm{I}_{12}^{2}+\mathrm{I}_{11} \mathrm{I}_{22}\right)}\left(-h I_{11} \mathrm{I}_{12}^{3} \mathrm{I}_{13}-h I_{11} \mathrm{I}_{12}^{2} \mathrm{I}_{11} \mathrm{I}_{23}+\mathrm{I}_{12} h I_{12} \mathrm{I}_{11}^{2} \mathrm{I}_{23}+h I_{22} \mathrm{I}_{11}^{2} \mathrm{I}_{12} \mathrm{I}_{13}-h I_{22} \mathrm{I}_{11}^{3} \mathrm{I}_{23}-\mathrm{I}_{11}^{2} \mathrm{I}_{22} \mathrm{I}_{13} h I_{12}+\mathrm{I}_{11}^{3} \mathrm{I}_{22} h I_{23}\right. \\
& \left.-\mathrm{I}_{12}^{2} h I_{23} \mathrm{I}_{11}^{2}+\mathrm{I}_{12}^{3} \mathrm{I}_{11} h I_{13}+2 \mathrm{I}_{11} \mathrm{I}_{22} \mathrm{I}_{12} h I_{11} \mathrm{I}_{13}-\mathrm{I}_{11}^{2} \mathrm{I}_{22} \mathrm{I}_{12} h I_{13}\right) \\
& j_{13}:=\operatorname{simplify}\left(\alpha_{5} \cdot \beta_{3}\right) \text {; } \\
& \frac{\left(-\mathrm{I}_{12} \mathrm{I}_{13}+\mathrm{I}_{11} \mathrm{I}_{23}\right)\left(h I_{11} \mathrm{I}_{12}^{2}-\mathrm{I}_{12} h I_{12} \mathrm{I}_{11}+h \mathrm{I}_{22} \mathrm{I}_{11}^{2}\right)}{\left(-\mathrm{I}_{12}^{2}+\mathrm{I}_{11} \mathrm{I}_{22}\right) \mathrm{I}_{11}^{2}} \\
& j_{14}:=\operatorname{simplify}\left(\alpha_{1}^{2}\right) \text {; } \\
& \mathrm{I}_{11} \\
& j_{15}:=\operatorname{simplify}\left(\alpha_{2}^{2}\right) \\
& \frac{\mathrm{I}_{12}^{2}}{\mathrm{I}_{11}} \\
& \frac{\mathrm{I}_{13}^{2}}{\mathrm{I}_{11}}
\end{aligned}
$$

$j_{17}:=\operatorname{simplify}\left(\beta_{1}^{2}\right) ;$

$$
\frac{h l_{11}^{2}}{\mathrm{I}_{11}}
$$

$j_{18}:=\operatorname{simplify}\left(\beta_{2}^{2}\right) ;$

$$
\frac{\left(-h I_{11} \mathrm{I}_{12}+h I_{12} \mathrm{I}_{11}\right)^{2}}{\mathrm{I}_{11}^{3}}
$$

$j_{19}:=\operatorname{simplify}\left(\beta_{4}^{2}\right) ;$

$$
\frac{\left(-h I_{11} \mathrm{I}_{13}+h I_{13} \mathrm{I}_{11}\right)^{2}}{\mathrm{I}_{11}^{3}}
$$

$j_{20}:=\operatorname{simplify}\left(\alpha_{1} \cdot \alpha_{2}\right)$;

$j_{21}:=\operatorname{simplify}\left(\alpha_{1} \cdot \alpha_{4}\right)$; 
$j_{22}:=\operatorname{simplify}\left(\alpha_{2} \cdot \alpha_{4}\right)$;

$$
\frac{\mathrm{I}_{12} \mathrm{I}_{13}}{\mathrm{I}_{11}}
$$

$j_{23}:=\operatorname{simplify}\left(\beta_{1} \cdot \beta_{2}\right) ;$

$$
\frac{h I_{11}\left(-h I_{11} \mathrm{I}_{12}+h I_{12} \mathrm{I}_{11}\right)}{\mathrm{I}_{11}^{2}}
$$

$j_{24}:=\operatorname{simplify}\left(\beta_{1} \cdot \beta_{4}\right)$;

$$
\frac{h I_{11}\left(-h I_{11} \mathrm{I}_{13}+h I_{13} \mathrm{I}_{11}\right)}{\mathrm{I}_{11}^{2}}
$$

$j_{25}:=\operatorname{simplify}\left(\beta_{4} \cdot \beta_{2}\right) ;$

$$
\frac{\left(-h I_{11} \mathrm{I}_{13}+h I_{13} \mathrm{I}_{11}\right)\left(-h I_{11} \mathrm{I}_{12}+h I_{12} \mathrm{I}_{11}\right)}{\mathrm{I}_{11}^{3}}
$$

$j_{26}:=\operatorname{simplify}\left(\alpha_{1} \cdot \beta_{1}\right) ;$

$$
h I_{11}
$$

$j_{27}:=\operatorname{simplify}\left(\alpha_{1} \cdot \beta_{2}\right) ;$

$$
\frac{-h I_{11} \mathrm{I}_{12}+h I_{12} \mathrm{I}_{11}}{\mathrm{I}_{11}}
$$

$j_{28}:=\operatorname{simplify}\left(\alpha_{1} \cdot \beta_{4}\right) ;$

$$
\frac{-h I_{11} \mathrm{I}_{13}+h I_{13} \mathrm{I}_{11}}{\mathrm{I}_{11}}
$$

$j_{29}:=\operatorname{simplify}\left(\alpha_{2} \cdot \beta_{1}\right)$;

$$
\frac{h I_{11} \mathrm{I}_{12}}{\mathrm{I}_{11}}
$$

$j_{30}:=\operatorname{simplify}\left(\alpha_{2} \cdot \beta_{2}\right)$;

$$
\frac{\mathrm{I}_{12}\left(-h I_{11} \mathrm{I}_{12}+h I_{12} \mathrm{I}_{11}\right)}{\mathrm{I}_{11}^{2}}
$$

$j_{31}:=\operatorname{simplify}\left(\alpha_{2} \cdot \beta_{4}\right) ;$

$$
\frac{\mathrm{I}_{12}\left(-h I_{11} \mathrm{I}_{13}+h I_{13} \mathrm{I}_{11}\right)}{\mathrm{I}_{11}^{2}}
$$

$j_{32}:=\operatorname{simplify}\left(\alpha_{4} \cdot \beta_{1}\right)$;

$$
\frac{h I_{11} \mathrm{I}_{13}}{\mathrm{I}_{11}}
$$

$j_{33}:=\operatorname{simplify}\left(\alpha_{4} \cdot \beta_{2}\right)$;

$$
\frac{\mathrm{I}_{13}\left(-h I_{11} \mathrm{I}_{12}+h I_{12} \mathrm{I}_{11}\right)}{\mathrm{I}_{11}^{2}}
$$

$j_{34}:=\operatorname{simplify}\left(\alpha_{4} \cdot \beta_{4}\right) ;$

$$
\frac{\mathrm{I}_{13}\left(-h I_{11} \mathrm{I}_{13}+h I_{13} \mathrm{I}_{11}\right)}{\mathrm{I}_{11}^{2}}
$$




\section{Bibliography}

[1] BAKER, A. Matrix groups: An introduction to Lie group theory. Springer, 2001.

[2] BALL, R. The theory of screws: A study in the dynamics of a rigid body. Mathematische Annalen 9 (1876), 541-553.

[3] BALL, R. A Treatise on the theory of screws. Cambridge University Press, 1900 .

[4] Brand, L. Vector and tensor analysis. J. Wiley, 1947.

[5] BrÖCKER, T., AND JÄNICH, K. Introduction to differential topology. Cambridge University Press, 1982.

[6] Brodsky, V., And Shoham, M. Dual numbers representation of rigid body dynamics. Mechanism and Machine Theory 34 (1999), 693-718.

[7] Chasles, M. Note sur les propriétés génerales du systéme de deux corps semblables entréux et placés dúne maniére quelconque dans léspace; et sur le déplacement fini ou infiniment petit dun corps solide libre : A note on the general properties of a system of two similar bodies arbitrarily positioned in space; and on the finite or infinitely small displacement of an unconstrained solid body. Bulletin des Sciences Mathématiques, Férussac 14 (1830), 321-26. 
[8] Chevallier, D. On the transference trinciple in kinematics; its various forms and limitations. Mechanism and Machine Theory 31 (1995), 5776.

[9] Chou, J. Quaternion kinematics and dynamic differential equations. IEEE Transaction on Robotics and Automation 8 (February 1992), 5364.

[10] Clifford, W. K. Preliminary sketch of bi-quaternions. Proc. London Math. Soc 4 (1873), 381-395.

[11] Cox, D., Little, J., And O’SheA, D. Ideals, Varieties, and Algorithms: An Introduction to computational algebraic geometry and commutative algebra, vol. 10 of Undergraduate Texts in Mathematics. Springer Verlag, 2007.

[12] Crook, D. Polynomial invariants of the Euclidean group action on multiple screws. Master's thesis, Victoria University of Wellington, 2009.

[13] Dixmier, J. Enveloping algebras, vol. 14. Newnes, 1977.

[14] Donelan, P., And Crook, D. Polynomial invariants and SAGBI bases for multi-screws. Victoria University of Wellington. Research Report (2010).

[15] Donelan, P., And Gibson, C. First-order invariants of Euclidean motions. Acta Applicandae Mathematica 24 (1991), 233-251.

[16] Donelan, P., ANd Selig, J. Introduction to polynomial invariants of screw systems. Victoria University of Wellington. Research Report (2007). 
[17] Duffy, J., And Rico, J. The principle of transference: History, statement and proof. Mechanism and Machine Theory 28 (1993), 165-177.

[18] ERdmann, K., AND Wildon, M. Introduction to Lie algebras. Springer, 2006.

[19] Fauske, K. An introduction to sketch 3d for pgf and tikz users, 2009. http://www.fauskes.net/nb/introduction-to-sketch.

[20] Fu, K., Gonzalez, R., And Lee, C. Robotics. Mcgraw-Hill International Editions, 1987.

[21] Goodman, R And Wallach, N. Representations and invariants of the classical groups, vol. 68. Cambridge University Press, 1998.

[22] Hall, B. An elementary introduction to groups and representations. arXiv preprint math-ph/0005032 (2000).

[23] Hartenberg, R., And Denavit, J. Kinematic synthesis of linkages. McGraw-Hill New York, 1964.

[24] Humphreys, J. Reflection groups and Coxeter groups, vol. 29. Cambridge University Press, 1992.

[25] Hunt, K. Kinematics geometry of mechanisms, vol. 68. Oxford, England: Clarendon Press, 1978.

[26] Husty, M. An algorithm for solving the direct kinematics of general stewart-gough platforms. Mechanism and Machine Theory 31 (1996), $365-379$.

[27] Jordan, C., And Jordan, D. Groups. Edward Arnold, 1994. 
[28] Kayal, N. The complexity of the annihilating polynomial. In Computational complexity, 2009. CCC'09. 24th Annual IEEE Conference on (2009), IEEE, pp. 184-193.

[29] Kirillov, A. Lectures on the orbit method, vol. 64. American Mathematical Soc, 2004.

[30] Kirillov, A. An introduction to Lie groups and Lie algebras, vol. 113. Cambridge University Press Cambridge, 2008.

[31] KRAFT, H. Algebraic tranformation groups, 2011. http://is.gd/mbpevX.

[32] Kreyszig, E. Advanced engineering mathematics. Wiley New York, NY, 1983.

[33] Macbeath, A. Elementary vector algebra, Second Edition, vol. 2. Oxford University Press, 1966.

[34] McCArthy, J. Dual orthogonal matrices in manipulator kinematics. The International Journal of Robotics Research 5 (1986), 45-51.

[35] McCarthy, J. An introduction to theoretical kinematics. The MIT Press, 1990.

[36] Nagata, M. On the 14-th problem of Hilbert. American Journal of Mathematics 81 (1959), 766-772.

[37] Neusel, M. Invariant theory, vol. 36 of Student Mathematical Library. AMS, 2006. 
[38] Newstead, P. Introduction to orbit spaces and moduli problems. Tata Institute of Fundamental Research Lectures on Mathematics and Physics, Springer-Verlag, New York, 1978.

[39] Panyushev, D. Semi-direct products of Lie algebras and their invariants. Publ. Res. Inst. Math. Sci. 4 (2007), 1199-1257.

[40] Paul, R. Robot manipulators: Mathematics, programming, and control. MIT Press, 1981.

[41] Pennestrì, E., And Stefanelli, R. Linear algebra and numerical algorithms using dual numbers. Multibody System Dynamics 18 (2007), $323-344$.

[42] PlüCker, J. On a new geometry of space. Philosophical Transactions of the Royal Society of London 155 (1865), 725-791.

[43] Rooney, J. A comparison of representations of general spatial screw displacement. Environment and Planning B 5 (1978), 45-88.

[44] Rooney, J. William Kingdon Clifford (1845-1879). In Distinguished Figures in Mechanism and Machine Science. Springer, 2007, pp. 79-116.

[45] Samuel, A., McAree, P., And Hunt, K. Unifying screw geometry and matrix transformations. The International Journal of Robotics Research 10 (1991), 454-472.

[46] Selig, J. Geometrical methods in robotics. Springer-Verlag New York, Inc, 1996.

[47] Selig, J. Geometric fundamentals of robotics. Springer, 2005. 
[48] Study, E. Geometrie der dynamen. Cornell Historical Mathematical Monographs (1903).

[49] Weyl, H. The classical groups: Their invariants and representations, vol. 1. Princeton University Press, 1997. 